\title{
Cobalt-Catalyzed Markovnikov-Selective Radical Hydroacylation of Unactivated Alkenes with
}

\section{Acylphosphonates}
Benxiang Zhang, ${ }^{\mathrm{b}}$ Jiayan $\mathrm{He},{ }^{\mathrm{b}}$ Yi Li, ${ }^{\mathrm{b}}$ Tao Song, ${ }^{\mathrm{b}}$ Yewen Fang, ${ }^{*, \mathrm{a}}$ and Chaozhong $\mathrm{Li}^{*}, \mathrm{a}, \mathrm{b}$

\begin{abstract}
${ }^{\text {a }}$ School of Materials and Chemical Engineering, Ningbo University of Technology, No. 201 Fenghua Road, Ningbo 315211, China, and ${ }^{\mathrm{b}}$ Key Laboratory of Organofluorine Chemistry, Center for Excellence in Molecular Synthesis, Shanghai Institute of Organic Chemistry, Chinese Academy of Sciences, 345 Lingling Road, Shanghai 200032, China
\end{abstract}

E-mail: fang@nbut.edu.cn (Y.F.); clig@mail.sioc.ac.cn (C.L.)

\section{Table of Contents}

1. General information 


\section{General information}

All commercially available compounds were purchased from Aldrich, Alfa Aesar or Adamas. Solvent was purified according to the procedure from "Purification of

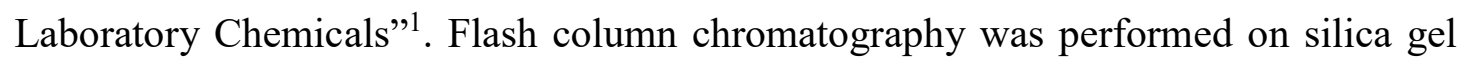
(particle size 300-400 mesh) and eluted with petroleum ether/ethyl acetate. Thin layer chromatography was performed on silica gel 60 F254 plates $(250 \mu \mathrm{m})$. NMR spectra were recorded on Bruker AV-400 instruments. The following abbreviations were used to explain the multiplicities: $\mathrm{s}=$ singlet, $\mathrm{d}=$ doublet, $\mathrm{t}=$ triplet, $\mathrm{q}=$ quartet, $\mathrm{m}=$ multiplet, br $=$ broad. IR spectra were recorded on a Bio Rad FTS-185 instrument. Melting points (mp) were recorded on a SGW X-4 apparatus and uncorrected. High-resolution mass spectra (HRMS) were recorded on a Finnigan MAT 8430, or an IonSpec 4.7 Tesla FT mass spectrometer.

\section{Tables S1-S3}

Table S1. Attempted Co/Ni-Dual Catalyzed Hydroacylation of Alkenes

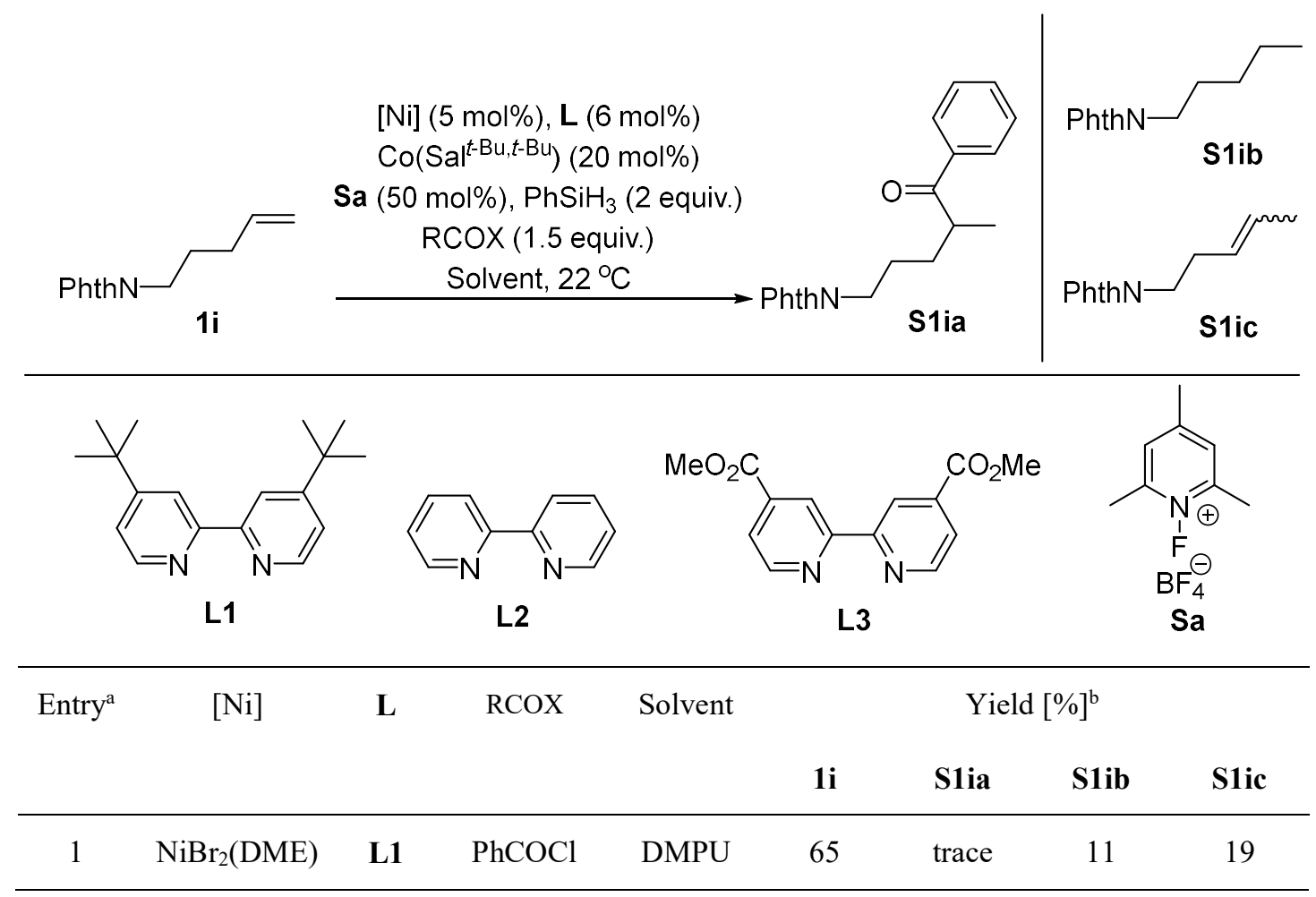




\begin{tabular}{ccccccccc}
\hline 2 & $\mathrm{Ni}(\mathrm{cod})_{2}$ & $\mathbf{L 1}$ & $\mathrm{PhCOCl}$ & $\mathrm{DMPU}$ & 63 & 0 & 13 & 15 \\
3 & $\mathrm{Ni}(\mathrm{acac})_{2}$ & $\mathbf{L 1}$ & $\mathrm{PhCOCl}$ & $\mathrm{DMPU}$ & 66 & 0 & 12 & 16 \\
4 & $\mathrm{NiBr}_{2}(\mathrm{DME})$ & $\mathbf{L 1}$ & $\mathrm{PhCOCl}$ & $\mathrm{DME}$ & 67 & 0 & 18 & 10 \\
5 & $\mathrm{NiBr}_{2}(\mathrm{DME})$ & $\mathbf{L 1}$ & $\mathrm{PhCOCl}$ & $\mathrm{THF}$ & 43 & trace & 21 & 17 \\
6 & $\mathrm{NiBr}_{2}(\mathrm{DME})$ & $\mathbf{L 2}$ & $\mathrm{PhCOCl}$ & $\mathrm{DMPU}$ & 68 & 0 & 12 & 16 \\
7 & $\mathrm{NiBr}_{2}(\mathrm{DME})$ & $\mathbf{L 3}$ & $\mathrm{PhCOCl}$ & $\mathrm{DMPU}$ & 61 & trace & 8 & 15 \\
8 & $\mathrm{NiBr}_{2}(\mathrm{DME})$ & $\mathbf{L 1}$ & $(\mathrm{PhCO})_{2} \mathrm{O}$ & $\mathrm{DMPU}$ & 50 & trace & 7 & 31 \\
9 & $\left.\mathrm{Ni}_{(\mathrm{acac}}\right)_{2}$ & $\mathbf{L 1}$ & $(\mathrm{PhCO})_{2} \mathrm{O}$ & $\mathrm{DME}$ & 59 & 0 & 12 & 20 \\
10 & $\mathrm{NiBr}_{2}(\mathrm{DME})$ & $\mathbf{L 3}$ & $(\mathrm{PhCO})_{2} \mathrm{O}$ & $\mathrm{THF}$ & 61 & 0 & 16 & 15 \\
\hline
\end{tabular}

${ }^{a}$ Reaction conditions: 1i $(0.15 \mathrm{mmol}),[\mathrm{Ni}](0.0075 \mathrm{mmol}), \mathbf{L}(0.0090 \mathrm{mmol}), \mathrm{Co}\left(\mathrm{Sal}^{t-\mathrm{Bu}, t-\mathrm{Bu}}\right)(0.030$ mmol), Sa (0.075 mmol), $\mathrm{PhSiH}_{3}(0.30 \mathrm{mmol}), \mathrm{RCOX}(0.23 \mathrm{mmol})$, solvent $(0.50 \mathrm{~mL}), 22{ }^{\circ} \mathrm{C}, 12$ h. ${ }^{\text {b }} \mathrm{H}$ NMR (400 MHz) yield based on $\mathbf{1 i}$.

Table S2. Optimization of Conditions for the Synthesis of 4a-Part 1

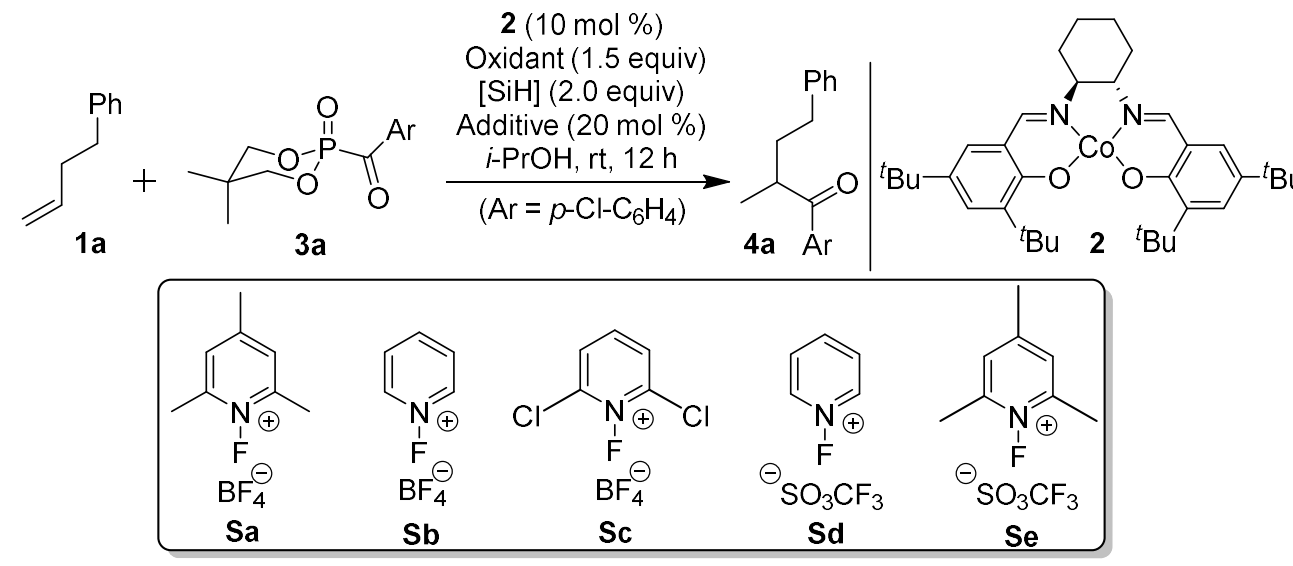

\begin{tabular}{ccccc}
\hline Entry $^{\mathrm{a}}$ & Oxidant & {$[\mathrm{SiH}]$} & Additive & ${\text { Yield [\% }]^{\mathrm{b}}}^{\mathrm{n}}$ \\
\hline 1 & $\mathrm{BIOH}$ & $\mathrm{PhSiH}_{3}$ & $/$ & 27 \\
2 & $\mathrm{BIOAc}$ & $\mathrm{PhSiH}_{3}$ & $/$ & 22 \\
3 & $\mathrm{DIB}$ & $\mathrm{PhSiH}_{3}$ & $/$ & 15 \\
4 & $\mathrm{PIFA}$ & $\mathrm{PhSiH}_{3}$ & $/$ & 8 \\
5 & $t$-BuOOH & $\mathrm{PhSiH}_{3}$ & $/$ & 60 \\
6 & $\mathrm{DTBP}$ & $\mathrm{PhSiH}_{3}$ & $/$ & 19 \\
7 & $\mathrm{BPO}$ & $\mathrm{PhSiH}_{3}$ & $/$ & 15 \\
8 & $\mathrm{DLP}$ & $\mathrm{PhSiH}_{3}$ & $/$ & 19 \\
\hline
\end{tabular}




\begin{tabular}{|c|c|c|c|c|}
\hline 9 & $t$-BuOOH & $\mathrm{Et}_{3} \mathrm{SiH}$ & / & trace \\
\hline 10 & $t$-BuOOH & $\mathrm{Ph}_{2} \mathrm{SiH}_{2}$ & / & 35 \\
\hline 11 & $t$-BuOOH & $\mathrm{Ph}(i-\mathrm{PrO}) \mathrm{SiH}_{2}$ & / & 56 \\
\hline 12 & $t$-BuOOH & $(\mathrm{MeO})_{2} \mathrm{MeSiH}$ & / & 13 \\
\hline 13 & $t$-BuOOH & $(\mathrm{MeO})_{3} \mathrm{SiH}$ & / & 17 \\
\hline 14 & $t-\mathrm{BuOOH}$ & $\left(\mathrm{Me}_{2} \mathrm{HSi}\right)_{2} \mathrm{O}$ & / & trace \\
\hline 15 & $t$-BuOOH & $\mathrm{PhSiH}_{3}$ & Sa & 74 \\
\hline 16 & $t$-BuOOH & $\mathrm{PhSiH}_{3}$ & Sb & 71 \\
\hline 17 & $t$-BuOOH & $\mathrm{PhSiH}_{3}$ & Sc & 62 \\
\hline 18 & $t$-BuOOH & $\mathrm{PhSiH}_{3}$ & Sd & 74 \\
\hline 19 & $t$-BuOOH & $\mathrm{PhSiH}_{3}$ & Se & 65 \\
\hline 20 & $t$-BuOOH & $\mathrm{PhSiH}_{3}$ & Selectfluor & 78 \\
\hline
\end{tabular}

${ }^{a}$ Reaction conditions: 1a $(0.20 \mathrm{mmol}), 2(0.020 \mathrm{mmol}), 3 \mathbf{a}(0.70 \mathrm{mmol})$, oxidant $(0.30 \mathrm{mmol})$, $[\mathrm{SiH}](0.40 \mathrm{mmol})$, additive $(0.040 \mathrm{mmol}), i-\mathrm{PrOH}(6.0 \mathrm{~mL}), 22^{\circ} \mathrm{C}, 12 \mathrm{~h} .{ }^{\mathrm{b}}$ Isolated yield based on 1 .

Table S3. Optimization of Conditions for the Synthesis of 4a-Part 2
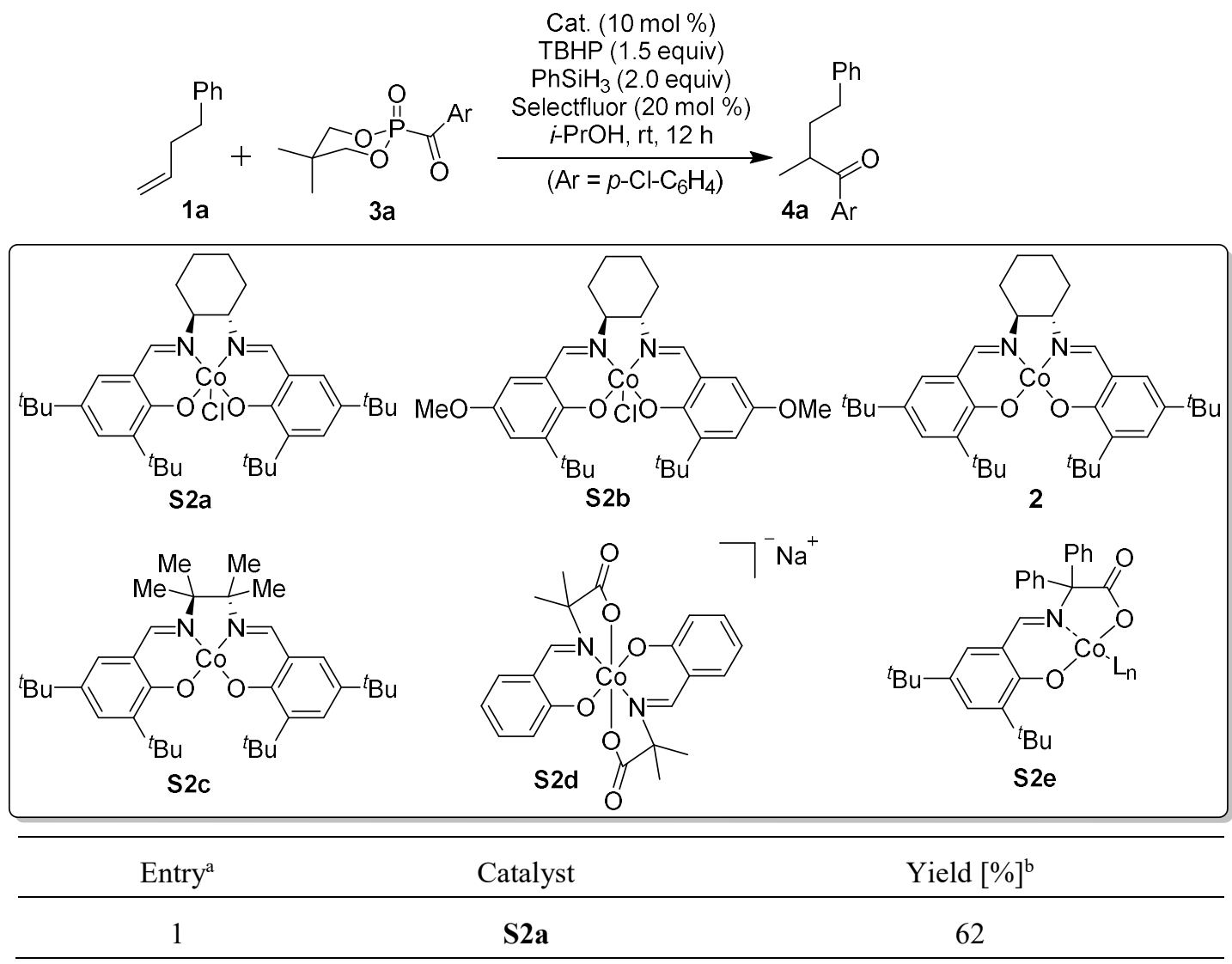


\begin{tabular}{lcc}
\hline 2 & S2b & 45 \\
3 & $\mathbf{2}$ & 78 \\
4 & $\mathbf{S 2 c}$ & 26 \\
5 & $\mathbf{S 2 d}$ & 5 \\
6 & $\mathbf{S 2 e}$ & trace \\
7 & $\mathrm{Fe}(\mathrm{acac})_{3}$ & 6 \\
8 & $\mathrm{Fe}(\mathrm{dpm})_{3}$ & 12 \\
9 & $\mathrm{Mn}(\mathrm{dpm})_{3}$ & 10 \\
\hline
\end{tabular}

${ }^{a}$ Reaction conditions: $1 \mathbf{a}(0.20 \mathrm{mmol})$, catalyst $(0.020 \mathrm{mmol}), \mathbf{3 a}(0.70 \mathrm{mmol})$, TBHP $(0.30 \mathrm{mmol})$, $\mathrm{PhSiH}_{3}(0.40 \mathrm{mmol})$, Selectfluor $(0.040 \mathrm{mmol}), i-\mathrm{PrOH}(6.0 \mathrm{~mL}), 22{ }^{\circ} \mathrm{C}, 12 \mathrm{~h} .{ }^{\mathrm{b}}$ Isolated yield based on 1a.

\section{Synthesis and characterizations of substrates}

The following substrates were purchased and directly used without further purification:

but-3-en-1-ylbenzene (1a), oct-1-ene (1b), tetradec-1-ene (1c), 11-chloroundec-1ene (1d), 8-bromooct-1-ene (1e), undec-10-enal (1k), cyclopentene (1w), cyclohexene (1x), methyl cyclopent-3-ene-1-carboxylate (1y).

The following substrates are known compounds:

pent-4-en-1-yl 4-methylbenzenesulfonate $(\mathbf{1 f})^{2}$,

$N$-(but-3-en-1-yl)-4-methylbenzenesulfonamide (1g) ${ }^{3}$,

(but-3-en-1-yloxy)(tert-butyl)diphenylsilane (1h) ${ }^{4}$,

2-(pent-4-en-1-yl)isoindoline-1,3-dione (1i) ${ }^{5}$,

7-nitrohept-1-ene $(\mathbf{1} \mathbf{j})^{2}$,

$N, N$-diethylundec-10-enamide (1I) ${ }^{6}$,

diethyl hept-6-en-1-ylphosphonate (1 m) ${ }^{7}$,

1-(but-3-en-1-yl)-4-(trifluoromethyl)benzene (1n) ${ }^{8}$,

3-(pent-4-en-1-yl)oxazolidin-2-one (1o) ${ }^{9}$,

pent-4-en-1-yl 2-(4-methoxyphenyl)acetate (1p) $)^{10}$, 
pent-4-en-1-yl 4-(tert-butyl)benzoate (1q) ${ }^{11}$,

pent-4-en-1-yl furan-2-carboxylate (1r) ${ }^{12}$,

pent-4-en-1-yl 2-naphthoate (1s) ${ }^{11}$,

4-(pent-4-en-1-yloxy)benzonitrile (1t) ${ }^{13}$,

3-methylbut-2-en-1-yl hex-5-enoate $(\mathbf{1} \mathbf{u})^{14}$,

cyclobutene $(\mathbf{1} \mathbf{v})^{15}$,

((3aS,5aR,8aR,8bS)-2,2,7,7-tetramethyltetrahydro-3a $H$-bis([1,3]dioxolo)[4,5-

$\left.b: 4^{\prime}, 5^{\prime}-d\right]$ pyran-3a-yl)methyl pent-4-enoate $(\mathbf{1 z b})^{16}$,

4-methyl-2-oxo-2H-chromen-7-yl hex-5-enoate (1zd) ${ }^{17}$,

$(8 R, 9 S, 13 S, 14 S)$-13-methyl-3-(pent-4-en-1-yloxy)-6,7,8,9,11,12,13,14,15,16-

decahydro-17H-cyclopenta[ $[a]$ phenanthren-17-one $(\mathbf{1 z f})^{11}$,

dimethyl (4-chlorobenzoyl)phosphonate $(\mathbf{3 c})^{18}$,

diethyl (4-chlorobenzoyl)phosphonate (3d) ${ }^{19}$,

vinylcyclopropane $(7)^{20}$,

dimethyl 2,2-diallylmalonate $(\mathbf{9})^{21}$.

\section{Preparation of Substrates}

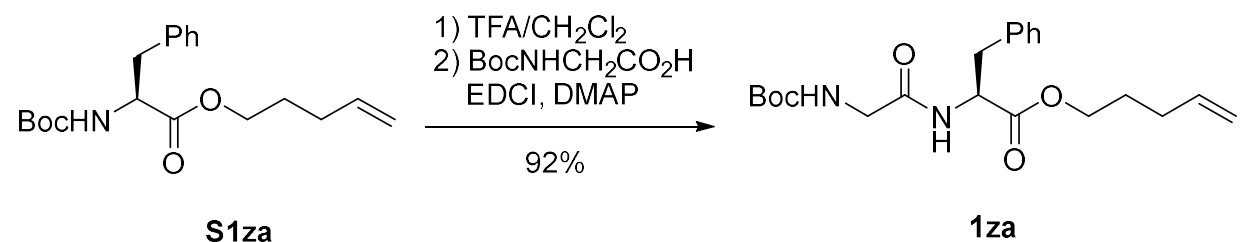

Pent-4-en-1-yl (tert-butoxycarbonyl)glycyl-L-phenylalaninate (1za). To a solution of $\mathbf{S 1 z a}^{22}(1.67 \mathrm{~g}, 5.0 \mathrm{mmol})$ in $\mathrm{CH}_{2} \mathrm{Cl}_{2}(15 \mathrm{~mL})$ was added TFA $(15 \mathrm{~mL})$ at room temperature (rt), and the mixture was stirred at rt for $1 \mathrm{~h}$. Saturated aqueous $\mathrm{NaHCO}_{3}$ was added at $0{ }^{\circ} \mathrm{C}$ to quench the reaction. The resulting mixture was extracted with $\mathrm{CH}_{2} \mathrm{Cl}_{2}(3 \times 20 \mathrm{~mL})$. The combined organic layers were washed with brine $(2 \times 60 \mathrm{~mL})$, dried over anhydrous $\mathrm{Na}_{2} \mathrm{SO}_{4}$, filtered, and concentrated under reduced pressure. The residue was dissolved in $\mathrm{CH}_{2} \mathrm{Cl}_{2}(24 \mathrm{~mL})$. BocNHCH $\mathrm{CO}_{2} \mathrm{H}(1.23 \mathrm{~g}, 7.0 \mathrm{mmol})$, EDCI $(1.35 \mathrm{~g}, 7.0 \mathrm{mmol})$ and DMAP $(854 \mathrm{mg}, 7.0 \mathrm{mmol})$ were added successively into the 
solution. The mixture was stirred at rt for $18 \mathrm{~h}$. Saturated aqueous $\mathrm{NaHCO}_{3}(30 \mathrm{~mL})$ was added to quench the reaction and the resulting mixture was extracted with $\mathrm{CH}_{2} \mathrm{Cl}_{2}$ $(3 \times 30 \mathrm{~mL})$. The combined organic layers were washed with brine $(2 \times 90 \mathrm{~mL})$, dried over anhydrous $\mathrm{Na}_{2} \mathrm{SO}_{4}$, filtered, and concentrated under reduced pressure. The residue was purified by flash column chromatography on silica gel with EtOAc/petroleum ether (1:2) as the eluent to give 1za as a colorless oil. Yield: $1.79 \mathrm{~g}$ (92\%). IR (neat): $v\left(\mathrm{~cm}^{-}\right.$ 1) $3323,3065,2977,2932,1739,1722,1682,1519,1455,1367,1278,1250,1172,1050$, 916, 865, 744, 701; ${ }^{1} \mathrm{H}$ NMR (400 MHz, $\left.\mathrm{CDCl}_{3}\right): \delta 7.18-7.30(\mathrm{~m}, 3 \mathrm{H}), 7.10(\mathrm{~d}, J=6.4$ $\mathrm{Hz}, 2 \mathrm{H}), 6.68(\mathrm{br}, 1 \mathrm{H}), 5.69-5.81(\mathrm{~m}, 1 \mathrm{H}), 5.22(\mathrm{br}, 1 \mathrm{H}), 4.95-5.05(\mathrm{~m}, 2 \mathrm{H}), 4.85(\mathrm{dd}$, $J=13.6,5.6 \mathrm{~Hz}, 1 \mathrm{H}), 4.01-4.15(\mathrm{~m}, 2 \mathrm{H}), 3.67-3.85(\mathrm{~m}, 2 \mathrm{H}), 3.04-3.15(\mathrm{~m}, 2 \mathrm{H})$, 1.95-2.08 (m, 2H), 1.62-1.72 (m, 2H), 1.43 (s, 9H) ppm; $\left.{ }^{13} \mathrm{C} \mathrm{NMR} \mathrm{(101} \mathrm{MHz,} \mathrm{CDCl}_{3}\right)$ : $\delta 171.3,169.1,155.9,137.1,135.6,129.2,128.5,127.1,115.4,80.1,64.9,53.1,44.1$, 37.9, 29.8, 28.2, 27.5 ppm; ESI-MS: $m / z$ 413.2 $\left(\mathrm{M}^{+}+\mathrm{Na}\right)$; HRMS calcd for $\mathrm{C}_{21} \mathrm{H}_{30} \mathrm{~N}_{2} \mathrm{NaO}_{5}[\mathrm{M}+\mathrm{Na}]:$ 413.2047, found 413.2045.

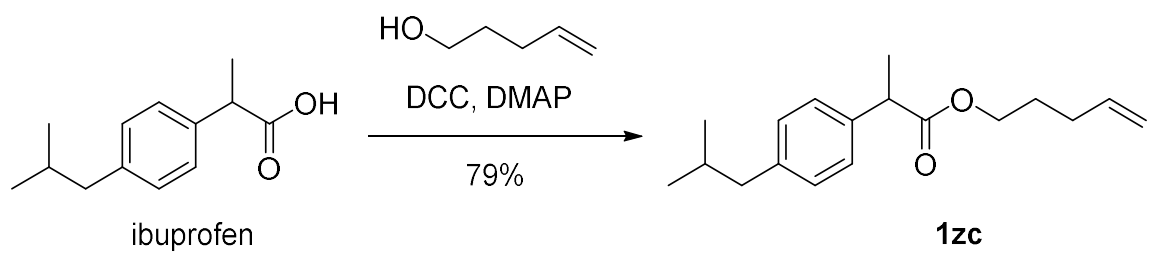

Pent-4-en-1-yl 2-(4-isobutylphenyl)propanoate (1zc). To a solution of ibuprofen (2.07 g, $10.0 \mathrm{mmol})$ in $\mathrm{CH}_{2} \mathrm{Cl}_{2}(20 \mathrm{~mL})$ was added DCC (5.16 g, $\left.25.0 \mathrm{mmol}\right)$ and DMAP (122 mg, $1.0 \mathrm{mmol})$ at $\mathrm{rt}$, and the mixture was stirred at $\mathrm{rt}$ for $12 \mathrm{~h}$. The resulting suspension was filtered through a short pad of celite with the aid of ethyl acetate (20 $\mathrm{mL}$ ). The filtrate was concentrated under reduced pressure. The residue was purified by flash column chromatography on silica gel with EtOAc/petroleum ether (1:200) as the eluent to give 1zc as colorless oil. Yield: $2.17 \mathrm{~g}(79 \%)$. IR (neat): $v\left(\mathrm{~cm}^{-1}\right) 3078,2955$, 1735, 1641, 1512, 1465, 1384, 1331, 1243, 1201, 1167, 1071, 914; ${ }^{1} \mathrm{H}$ NMR (400 MHz, $\left.\mathrm{CDCl}_{3}\right): \delta 7.21(\mathrm{~d}, J=8.0 \mathrm{~Hz}, 2 \mathrm{H}), 7.10(\mathrm{~d}, J=8.0 \mathrm{~Hz}, 2 \mathrm{H}), 5.67-5.79(\mathrm{~m}, 1 \mathrm{H})$, $4.90-4.98(\mathrm{~m}, 2 \mathrm{H}), 4.07$ (t, $J=6.4 \mathrm{~Hz}, 2 \mathrm{H}), 3.69(\mathrm{q}, J=7.2 \mathrm{~Hz}, 1 \mathrm{H}), 2.45(\mathrm{~d}, J=6.8$ $\mathrm{Hz}, 2 \mathrm{H}), 1.97-2.05(\mathrm{~m}, 2 \mathrm{H}), 1.77-1.91(\mathrm{~m}, 1 \mathrm{H}), 1.63-1.72(\mathrm{~m}, 2 \mathrm{H}), 1.49$ (d, $J=7.2$ 
$\mathrm{Hz}, 3 \mathrm{H}), 0.90(\mathrm{~d}, J=6.8 \mathrm{~Hz}, 6 \mathrm{H}) \mathrm{ppm} ;{ }^{13} \mathrm{C} \mathrm{NMR}\left(101 \mathrm{MHz}, \mathrm{CDCl}_{3}\right): \delta 174.7,140.4$, $137.8,137.4,129.3,127.1,115.2,63.9,45.2,45.0,30.2,29.9,27.7,22.3,18.4$ ppm; ESI-MS: $m / z 275.2\left(\mathrm{M}^{+}+\mathrm{H}\right)$; HRMS calcd for $\mathrm{C}_{18} \mathrm{H}_{27} \mathrm{O}_{2}[\mathrm{M}+\mathrm{H}]: 275.2006$, found 275.2004 .

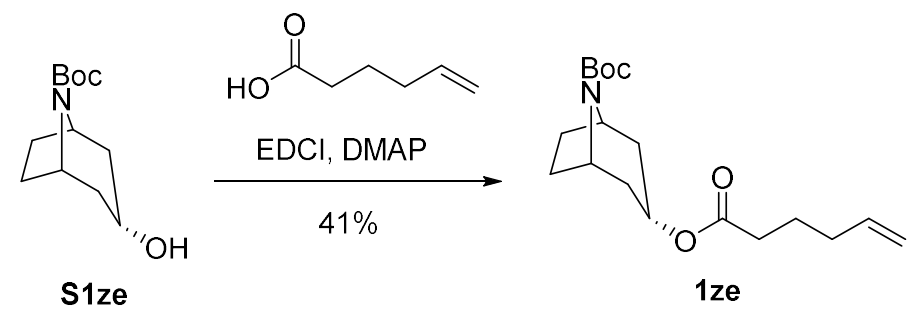

tert-Butyl $(1 R, 3 S, 5 S)-3-(h e x-5$-enoyloxy)-8-azabicyclo[3.2.1]octane-8-carboxylate (1ze). To a solution of S1ze $(682 \mathrm{mg}, 3.0 \mathrm{mmol})$ in $\mathrm{CH}_{2} \mathrm{Cl}_{2}(15 \mathrm{~mL})$ was added EDCI $(1.15 \mathrm{~g}, 6.0 \mathrm{mmol})$ and DMAP $(73.2 \mathrm{mg}, 0.60 \mathrm{mmol})$ at $\mathrm{rt}$, and the mixture was stirred at $\mathrm{rt}$ for $12 \mathrm{~h}$. Saturated aqueous $\mathrm{NaHCO}_{3}(30 \mathrm{~mL})$ was added to quench the reaction and the resulting mixture was extracted with $\mathrm{CH}_{2} \mathrm{Cl}_{2}(3 \times 20 \mathrm{~mL})$. The combined organic layers were washed with brine $(2 \times 60 \mathrm{~mL})$, dried over anhydrous $\mathrm{Na}_{2} \mathrm{SO}_{4}$, filtered, and concentrated under reduced pressure. The residue was purified by flash column chromatography on silica gel with EtOAc/petroleum ether (1:10) as the eluent to give 1ze as a colorless oil. Yield: $400 \mathrm{mg}(41 \%)$. IR (neat): $v\left(\mathrm{~cm}^{-1}\right)$ 3077, 2975, 1732 , 1695, 1478, 1393, 1366, 1328, 1244, 1163, 1079, 1033; ${ }^{1} \mathrm{H}$ NMR (400 MHz, $\left.\mathrm{CDCl}_{3}\right)$ : $\delta 5.70-5.83(\mathrm{~m}, 1 \mathrm{H}), 4.94-5.11(\mathrm{~m}, 3 \mathrm{H}), 4.05-4.30(\mathrm{~m}, 2 \mathrm{H}), 2.30(\mathrm{t}, J=7.6 \mathrm{~Hz}, 2 \mathrm{H})$, 2.02-2.21 (m, 4H), 1.89-2.01 (m, 4H), 1.65-1.81 (m, 4H), $1.44(\mathrm{~s}, 9 \mathrm{H}) \mathrm{ppm} ;{ }^{13} \mathrm{C}$ NMR $\left(101 \mathrm{MHz}, \mathrm{CDCl}_{3}\right): \delta 172.6,153.3,137.5,115.4,79.3,67.7,52.6,51.8,35.8,35.1,34.1$, 33.0, 28.4, 28.3, 27.6, 23.9 ppm; ESI-MS: m/z $346.2\left(\mathrm{M}^{+}+\mathrm{Na}\right)$; HRMS calcd for $\mathrm{C}_{18} \mathrm{H}_{29} \mathrm{NNaO}_{4}[\mathrm{M}+\mathrm{Na}]: 346.1989$, found 346.1987. 


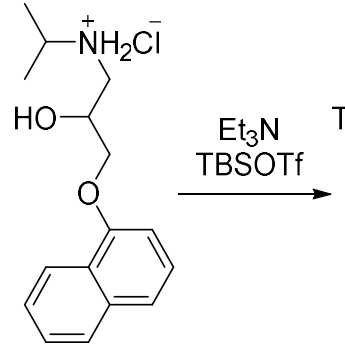

propranolol hydrochloride

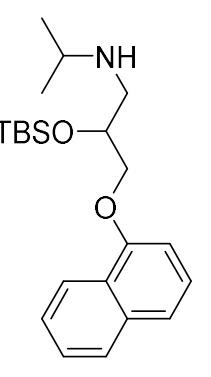

S5a-1
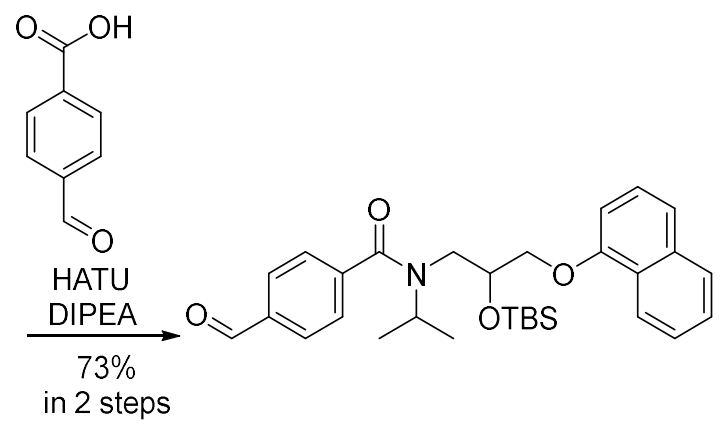

S5a-2

\section{$N$-(2-((tert-Butyldimethylsilyl)oxy)-3-(naphthalen-1-yloxy)propyl)-4-formyl- $N$ -}

isopropylbenzamide (S5a-2). To a solution of propranolol hydrochloride (2.96 g, 10.0 mmol) in $\mathrm{CH}_{2} \mathrm{Cl}_{2}(50 \mathrm{~mL})$ was added $\mathrm{Et}_{3} \mathrm{~N}(5.6 \mathrm{~mL}, 40.0 \mathrm{mmol})$ and TBSOTf (4.6 mL, $20.0 \mathrm{mmol}$ ) at $\mathrm{rt}$, and the mixture was stirred at $\mathrm{rt}$ for $12 \mathrm{~h}$. Saturated aqueous $\mathrm{NaHCO}_{3}$ $(50 \mathrm{~mL})$ was added to quench the reaction. The resulting mixture was extracted with $\mathrm{CH}_{2} \mathrm{Cl}_{2}(3 \times 50 \mathrm{~mL})$. The combined organic layers were washed with brine $(2 \times 1500$ $\mathrm{mL}$ ), dried over anhydrous $\mathrm{Na}_{2} \mathrm{SO}_{4}$, filtered, and concentrated under reduced pressure. The residue was dissolved in $\mathrm{CH}_{3} \mathrm{CN}$ (50 mL). 4-Formylbenzoic acid (1.50 g, $\left.10 \mathrm{mmol}\right)$, DIPEA (4.2 mL, $25.0 \mathrm{mmol})$ and HATU (3.80 m, $10.0 \mathrm{mmol})$ were added successively into the solution. The mixture was stirred at rt for $12 \mathrm{~h}$. Saturated aqueous $\mathrm{NaHCO}_{3}(50$ $\mathrm{mL}$ ) was added to quench the reaction and the resulting mixture was extracted with $\mathrm{CH}_{2} \mathrm{Cl}_{2}(3 \times 50 \mathrm{~mL})$. The combined organic layers were washed with brine $(2 \times 150$ $\mathrm{mL}$ ), dried over anhydrous $\mathrm{Na}_{2} \mathrm{SO}_{4}$, filtered, and concentrated under reduced pressure. The residue was purified by flash column chromatography on silica gel with EtOAc/petroleum ether (1:5) as the eluent to give S5a-2 as a colorless oil. Yield: 3.69 g (73\%). IR (neat): $v\left(\mathrm{~cm}^{-1}\right) 3053,2955,2929,2856,1705,1633,1580,1508,1462$, 1404, 1269, 1103, 910, 837, 773, 733; ${ }^{1} \mathrm{H}$ NMR (400 MHz, $\left.\mathrm{CDCl}_{3}\right): \delta 10.04(\mathrm{~s}, 1 \mathrm{H})$, $8.36(\mathrm{~d}, J=6.8 \mathrm{~Hz}, 1 \mathrm{H}), 7.92(\mathrm{~d}, J=7.6 \mathrm{~Hz}, 2 \mathrm{H}), 7.81(\mathrm{~d}, J=7.2 \mathrm{~Hz}, 1 \mathrm{H}), 7.42-7.59$ (m, 5H), $7.38(\mathrm{t}, J=7.6 \mathrm{~Hz}, 1 \mathrm{H}), 6.84(\mathrm{~d}, J=7.2 \mathrm{~Hz}, 1 \mathrm{H}), 4.77-4.89(\mathrm{~m}, 1 \mathrm{H}), 4.12-4.28$ (m, 2H), 3.88-4.01 (m, 1H), $3.80(\mathrm{dd}, J=13.2,6.4 \mathrm{~Hz}, 1 \mathrm{H}), 3.48(\mathrm{dd}, J=13.2,6.4 \mathrm{~Hz}$, 1H), 1.25 (d, $J=6.4 \mathrm{~Hz}, 3 \mathrm{H}), 1.13(\mathrm{~d}, J=6.4 \mathrm{~Hz}, 3 \mathrm{H}), 0.94(\mathrm{~s}, 9 \mathrm{H}), 0.23(\mathrm{~s}, 3 \mathrm{H}), 0.16$ (s, 3H) ppm; ${ }^{13} \mathrm{C}$ NMR (101 MHz, $\left.\mathrm{CDCl}_{3}\right): \delta 191.4,171.6,154.6,142.8,136.7,134.5$, 
$129.9,127.4,127.0,126.4,125.8,125.6,125.2,122.1,120.5,104.7,71.2,68.4,50.9$, 45.1, 25.8, 21.4, 21.0, 18.0, -4.3, -4.6 ppm; ESI-MS: $m / z 506.3\left(\mathrm{M}^{+}+\mathrm{H}\right)$; HRMS calcd for $\mathrm{C}_{30} \mathrm{H}_{40} \mathrm{NO}_{4} \mathrm{Si}[\mathrm{M}+\mathrm{H}]: 506.2721$, found 506.2719.

\section{Preparation of acylphosphonates}

Typical procedure for the preparations of $3 \mathrm{a}, 3 \mathrm{~b}, 3 \mathrm{e}, 3 \mathrm{f}, 3 \mathrm{~g}, 3 \mathrm{~h}$ and $5 \mathrm{a}-5 \mathrm{p}$

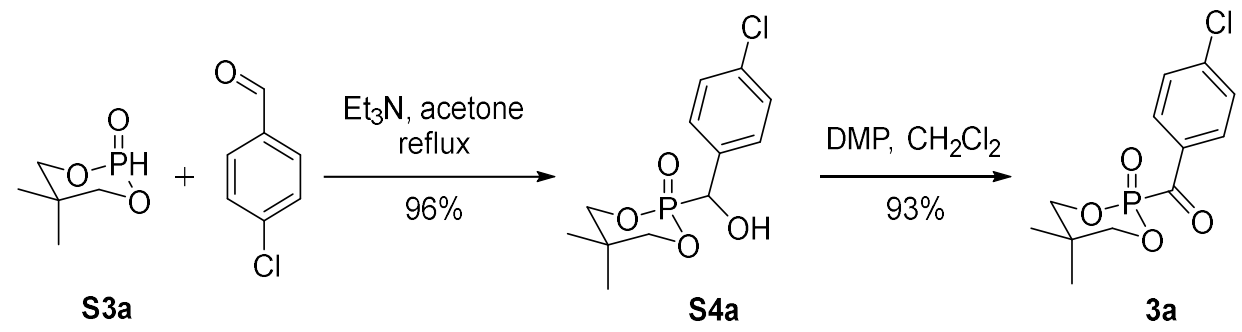

To a solution of 4-chlorobenzaldehyde $(700 \mathrm{mg}, 5.0 \mathrm{mmol})$ in acetone $(2.5 \mathrm{~mL})$ was added 5,5-dimethyl-1,3,2-dioxaphosphinane 2-oxide $\mathbf{S 3 a ^ { 2 3 }}(825 \mathrm{mg}, 5.5 \mathrm{mmol})$ and $\mathrm{Et}_{3} \mathrm{~N}(0.35 \mathrm{~mL}, 2.5 \mathrm{mmol})$. The solution was stirred at reflux for $2 \mathrm{~h}$. After cooled down to $\mathrm{rt}$, the solution was concentrated under reduced pressure. The residue was precipitated in $\mathrm{Et}_{2} \mathrm{O}(20 \mathrm{ml})$ and $\mathrm{EtOH}(0.20 \mathrm{~mL})$ to ascertain alcohol $\mathbf{S 4 a}$ as a white solid. Yield: $1.46 \mathrm{~g}(96 \%)$.

To a solution of $\mathbf{S 4 a}(1.16 \mathrm{~g}, 4.0 \mathrm{mmol})$ in $\mathrm{CH}_{2} \mathrm{Cl}_{2}(16 \mathrm{~mL})$ was added DMP $(2.55 \mathrm{~g}$, $6.0 \mathrm{mmol}$ ) in 3 portions and the mixture was stirred at $\mathrm{rt}$ for $2 \mathrm{~h}$. The reaction was quenched with saturated aqueous $\mathrm{Na}_{2} \mathrm{SO}_{3}(30 \mathrm{~mL})$ and the resulting mixture extracted with $\mathrm{CH}_{2} \mathrm{Cl}_{2}(3 \times 20 \mathrm{~mL})$. The combined organic layers were washed with saturated aqueous $\mathrm{Na}_{2} \mathrm{SO}_{3}(4 \times 60 \mathrm{~mL})$, saturated aqueous $\mathrm{NaHCO}_{3}(4 \times 60 \mathrm{~mL})$ and brine $(60$ $\mathrm{mL}$ ), dried over anhydrous $\mathrm{Na}_{2} \mathrm{SO}_{4}$, filtered, and concentrated under reduced pressure, the ketone 3a was thus obtained as a white solid and directly used without further purification. Yield: $1.07 \mathrm{~g}(93 \%)$.

\section{Characterization of new acylphosphonates}




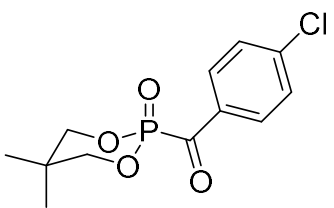

(4-Chlorophenyl)(5,5-dimethyl-2-oxido-1,3,2-dioxaphosphinan-2-yl)methanone

(3a). 93\% yield; White solid, m.p.: $105-107^{\circ} \mathrm{C}$; IR (neat): $v\left(\mathrm{~cm}^{-1}\right) 3091,3072,2972$, 2898, 1649, 1584, 1474, 1403, 1374, 1281, 1226, 1059, 1013, 985, 844, 779; ${ }^{1} \mathrm{H}$ NMR (400 MHz, $\left.\mathrm{CDCl}_{3}\right): \delta 8.32(\mathrm{~d}, J=8.4 \mathrm{~Hz}, 2 \mathrm{H}), 7.48(\mathrm{~d}, J=8.4 \mathrm{~Hz}, 2 \mathrm{H}), 4.39(\mathrm{~d}, J=$ $10.4 \mathrm{~Hz}, 2 \mathrm{H}), 4.05$ (dd, $J=18.8,10.8 \mathrm{~Hz}, 2 \mathrm{H}), 1.38$ (s, 3H), $0.90(\mathrm{~s}, 3 \mathrm{H}) \mathrm{ppm} ;{ }^{31} \mathrm{P}$ NMR (163 MHz, $\left.\mathrm{CDCl}_{3}\right): \delta-11.1 \mathrm{ppm} ;{ }^{13} \mathrm{C} \mathrm{NMR}\left(101 \mathrm{MHz}, \mathrm{CDCl}_{3}\right): \delta 199.3$ (d, $J=170.6$ Hz), 142.0, 133.7 (d, $J=63.2 \mathrm{~Hz}), 131.5,129.3,79.2$ (d, $J=8.2 \mathrm{~Hz}), 32.8$ (d, $J=8.3$ Hz), 22.0, 20.3 ppm; ESI-MS: m/z $310.9\left(\mathrm{M}^{+}+\mathrm{Na}\right)$; HRMS calcd for $\mathrm{C}_{12} \mathrm{H}_{15} \mathrm{ClO}_{4} \mathrm{P}$ [M+H]: 289.0391, found 289.0390.

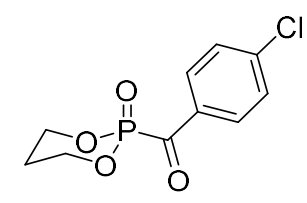

(4-Chlorophenyl)(2-oxido-1,3,2-dioxaphosphinan-2-yl)methanone (3b). The reaction was conducted using 1,3,2-dioxaphosphinane 2-oxide $\mathbf{S 3} \mathbf{b}^{24} .86 \%$ yield; White solid, m.p.: 103-105 ${ }^{\circ} \mathrm{C}$; IR (neat): $v\left(\mathrm{~cm}^{-1}\right)$ 3091, 2974, 2923, 1648, 1584, 1486, 1403 , 1307, 1282, 1236, 1138, 1057, 967, 929, 871, 758; ${ }^{1} \mathrm{H}$ NMR (400 MHz, $\left.\mathrm{CDCl}_{3}\right): \delta 8.32$ $(\mathrm{dd}, J=8.8,2.0 \mathrm{~Hz}, 2 \mathrm{H}), 7.48(\mathrm{~d}, J=8.8 \mathrm{~Hz}, 2 \mathrm{H}), 4.67$ (t, $J=11.6 \mathrm{~Hz}, 2 \mathrm{H}), 4.48-4.60$ (m, 2H), 2.37-2.52 (m, 1H), 1.84-1.95 (m, 1H) ppm; ${ }^{31} \mathrm{P}$ NMR (163 MHz, $\left.\mathrm{CDCl}_{3}\right): \delta$ -10.7 ppm; ${ }^{13} \mathrm{C}$ NMR (101 MHz, $\left.\mathrm{CDCl}_{3}\right): \delta 199.5$ (d, $\left.J=171.4 \mathrm{~Hz}\right), 142.0,133.7$ (d, $J$ $=64.1 \mathrm{~Hz}), 131.5(\mathrm{~d}, J=1.8 \mathrm{~Hz}), 129.3,70.1(\mathrm{~d}, J=8.2 \mathrm{~Hz}), 26.3(\mathrm{~d}, J=10.0 \mathrm{~Hz}) \mathrm{ppm}$; ESI-MS: $m / z 283.0\left(\mathrm{M}^{+}+\mathrm{Na}\right)$; HRMS calcd for $\mathrm{C}_{10} \mathrm{H}_{11} \mathrm{ClO}_{4} \mathrm{P}[\mathrm{M}+\mathrm{H}]: 261.0078$, found 261.0077.

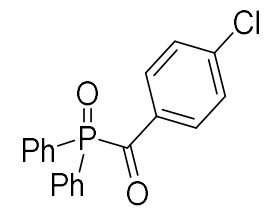


(4-Chlorophenyl)(diphenylphosphoryl)methanone (3e). The reaction was conducted using diphenylphosphine oxide S3e. 91\% yield; Light yellow oil; IR (neat): $v\left(\mathrm{~cm}^{-1}\right)$ $3058,2924,1708,1647,1583,1485,1438,1218,1176,1115,1014,943,729 ;{ }^{1} \mathrm{H}$ NMR $\left(400 \mathrm{MHz}, \mathrm{CDCl}_{3}\right): \delta 8.48(\mathrm{~d}, J=8.4 \mathrm{~Hz}, 2 \mathrm{H}), 7.80-7.90(\mathrm{~m}, 4 \mathrm{H}), 7.51-7.57(\mathrm{~m}, 2 \mathrm{H})$, 7.38-7.50 (m, 6H) ppm; ${ }^{31} \mathrm{P}$ NMR (163 MHz, $\left.\mathrm{CDCl}_{3}\right): \delta 22.8 \mathrm{ppm} ;{ }^{13} \mathrm{C}$ NMR $(101$ $\left.\mathrm{MHz}, \mathrm{CDCl}_{3}\right): \delta 203.6(\mathrm{~d}, J=82.1 \mathrm{~Hz}), 141.7,135.1(\mathrm{~d}, J=47.4 \mathrm{~Hz}), 132.6(\mathrm{~d}, J=3.6$ $\mathrm{Hz}), 131.8(\mathrm{~d}, J=9.1 \mathrm{~Hz}), 131.6,129.6(\mathrm{~d}, J=97.6 \mathrm{~Hz}), 129.3,128.7(\mathrm{~d}, J=11.8 \mathrm{~Hz})$ ppm; ESI-MS: $m / z 363.0\left(\mathrm{M}^{+}+\mathrm{Na}\right)$; HRMS calcd for $\mathrm{C}_{19} \mathrm{H}_{15} \mathrm{ClO}_{2} \mathrm{P}[\mathrm{M}+\mathrm{H}]$ : 341.0493, found 341.0490 .

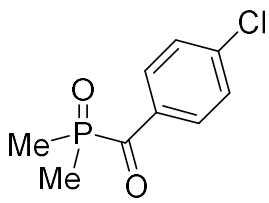

(4-chlorophenyl)(dimethylphosphoryl)methanone (3f). The reaction was conducted using dimethylphosphine oxide S3f. 89\% yield; White solid, m.p.: $67-69^{\circ} \mathrm{C}$; IR (neat): $v\left(\mathrm{~cm}^{-1}\right) 3088,2923,2854,1650,1584,1486,1402,1290,1224,1176,1089,1014,917$, 869, 739; ${ }^{1} \mathrm{H} \mathrm{NMR}\left(400 \mathrm{MHz}, \mathrm{CDCl}_{3}\right): \delta 8.47$ (d, $\left.J=8.8 \mathrm{~Hz}, 2 \mathrm{H}\right), 7.50$ (d, $J=8.8 \mathrm{~Hz}$, 1H), 1.75 (d, $J=12.8 \mathrm{~Hz}, 6 \mathrm{H}) \mathrm{ppm} ;{ }^{31} \mathrm{P}$ NMR (163 MHz, $\left.\mathrm{CDCl}_{3}\right): \delta 40.6 \mathrm{ppm} ;{ }^{13} \mathrm{C}$ NMR (125 MHz, $\left.\mathrm{CDCl}_{3}\right): \delta 205.3(\mathrm{~d}, J=76.5 \mathrm{~Hz}), 141.9,134.3(\mathrm{~d}, J=44.6 \mathrm{~Hz}), 131.4$, 129.5, $15.7(\mathrm{~d}, J=66.5 \mathrm{~Hz})$ ppm; ESI-MS: $m / z 217.0\left(\mathrm{M}^{+}+\mathrm{H}\right)$; HRMS calcd for $\mathrm{C}_{9} \mathrm{H}_{11} \mathrm{O}_{2} \mathrm{ClP}[\mathrm{M}+\mathrm{H}]: 217.0180$, found 217.0180.<smiles>CCOP(=O)(C(=O)c1ccc(Cl)cc1)c1ccccc1</smiles>

Ethyl (4-chlorobenzoyl)(phenyl)phosphinate (3g). The reaction was conducted using ethyl phenylphosphinate S3g. 92\% yield; Light yellow oil; IR (neat): v ( $\left.\mathrm{cm}^{-1}\right) 3062$, 2982, 2926, 2855, 1652, 1583, 1485, 1439, 1234, 1121, 1025, 930, 839, 778; ${ }^{1} \mathrm{H}$ NMR $\left(400 \mathrm{MHz}, \mathrm{CDCl}_{3}\right): \delta 8.35(\mathrm{~d}, J=8.4 \mathrm{~Hz}, 2 \mathrm{H}), 7.84-7.92(\mathrm{~m}, 2 \mathrm{H}), 7.59(\mathrm{t}, J=7.6 \mathrm{~Hz}$, 1H), 7.54-7.46 (m, 2H), 7.45 (d, $J=8.4 \mathrm{~Hz}, 2 \mathrm{H}), 4.15-4.33(\mathrm{~m}, 2 \mathrm{H}), 1.40$ (t, $J=7.2$ 
$\mathrm{Hz}, 3 \mathrm{H}) \mathrm{ppm} ;{ }^{31} \mathrm{P}$ NMR $\left(163 \mathrm{MHz}, \mathrm{CDCl}_{3}\right): \delta 21.0 \mathrm{ppm} ;{ }^{13} \mathrm{C} \mathrm{NMR}\left(125 \mathrm{MHz}, \mathrm{CDCl}_{3}\right)$ : $\delta 200.7$ (d, $J=118.4 \mathrm{~Hz}), 141.4,134.2$ (d, $J=54.8 \mathrm{~Hz}), 133.3$ (d, $J=2.8 \mathrm{~Hz}), 132.5$ (d, $J=10.0 \mathrm{~Hz}), 131.4,129.2,128.7$ (d, $J=12.5 \mathrm{~Hz}), 127.3$ (d, $J=129.4 \mathrm{~Hz}), 62.7$ (d, $J=$ $7.4 \mathrm{~Hz}), 16.5(\mathrm{~d}, J=6.4 \mathrm{~Hz})$ ppm; ESI-MS: $m / z 309.0\left(\mathrm{M}^{+}+\mathrm{H}\right)$; HRMS calcd for $\mathrm{C}_{15} \mathrm{H}_{15} \mathrm{O}_{3} \mathrm{ClP}[\mathrm{M}+\mathrm{H}]: 309.0442$, found 309.0442 .

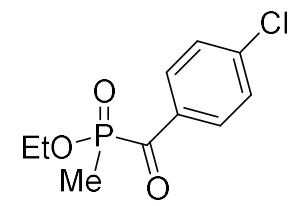

Ethyl (4-chlorobenzoyl)(methyl)phosphinate (3h). The reaction was conducted using ethyl phenylphosphinate $\mathbf{S 3 h}{ }^{25}$. 92\% yield; Colorless oil; IR (neat): $v\left(\mathrm{~cm}^{-1}\right)$ 3075, 2960, 2924, 2854, 1649, 1585, 1486, 1402, 1299, 1232, 1176, 1034, 969, 878, 784; ${ }^{1} \mathrm{H}$ NMR $\left(400 \mathrm{MHz}, \mathrm{CDCl}_{3}\right): \delta 8.35(\mathrm{~d}, J=8.0 \mathrm{~Hz}, 2 \mathrm{H}), 7.48(\mathrm{~d}, J=8.0 \mathrm{~Hz}, 2 \mathrm{H}), 4.01-4.26(\mathrm{~m}$, 2H), 1.74 (d, $J=14.4 \mathrm{~Hz}, 3 \mathrm{H}), 1.30$ (t, $J=7.2 \mathrm{~Hz}, 3 \mathrm{H}) \mathrm{ppm} ;{ }^{31} \mathrm{P}$ NMR $(163 \mathrm{MHz}$, $\left.\mathrm{CDCl}_{3}\right): \delta 36.7 \mathrm{ppm} ;{ }^{13} \mathrm{C} \mathrm{NMR}\left(125 \mathrm{MHz}, \mathrm{CDCl}_{3}\right): \delta 201.6(\mathrm{~d}, J=112.9 \mathrm{~Hz}), 141.6$, $134.2(\mathrm{~d}, J=51.9 \mathrm{~Hz}), 131.2,129.3,62.1$ (d, $J=6.4 \mathrm{~Hz}), 16.4$ (d, $J=5.4 \mathrm{~Hz}), 12.2$ (d, $J=96.6 \mathrm{~Hz})$ ppm; ESI-MS: $m / z 247.0\left(\mathrm{M}^{+}+\mathrm{H}\right)$; HRMS calcd for $\mathrm{C}_{10} \mathrm{H}_{13} \mathrm{O}_{3} \mathrm{ClP}[\mathrm{M}+\mathrm{H}]$ : 247.0285, found 247.0287.

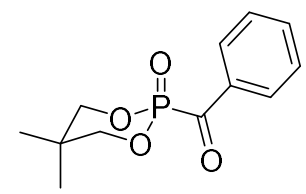

(5,5-Dimethyl-2-oxido-1,3,2-dioxaphosphinan-2-yl)(phenyl)methanone (5a). 95\% yield; White solid, m.p.: $84-86{ }^{\circ} \mathrm{C}$; IR (neat): $v\left(\mathrm{~cm}^{-1}\right)$ 2922, 1647, 1595, 1507, 1472, 1276, 1056, 983, 835, 772; ${ }^{1} \mathrm{H}$ NMR (400 MHz, $\left.\mathrm{CDCl}_{3}\right): \delta 8.34$ (d, $\left.J=7.6 \mathrm{~Hz}, 2 \mathrm{H}\right)$, $7.65(\mathrm{t}, J=7.6 \mathrm{~Hz}, 1 \mathrm{H}), 7.51(\mathrm{t}, J=7.6 \mathrm{~Hz}, 2 \mathrm{H}), 4.37$ (d, $J=10.8 \mathrm{~Hz}, 2 \mathrm{H}), 4.04$ (dd, $J$ $=19.2,11.2 \mathrm{~Hz}, 2 \mathrm{H}), 1.37(\mathrm{~s}, 3 \mathrm{H}), 0.88(\mathrm{~s}, 3 \mathrm{H}) \mathrm{ppm} ;{ }^{31} \mathrm{P} \mathrm{NMR}\left(163 \mathrm{MHz}, \mathrm{CDCl}_{3}\right): \delta-$ $10.4 \mathrm{ppm} ;{ }^{13} \mathrm{C}$ NMR $\left(101 \mathrm{MHz}, \mathrm{CDCl}_{3}\right): \delta 200.3$ (d, $\left.J=168.0 \mathrm{~Hz}\right), 135.3$ (d, $J=62.3$ $\mathrm{Hz}), 135.2,130.1$ (d, $J=1.6 \mathrm{~Hz}), 128.9,79.1$ (d, $J=7.7 \mathrm{~Hz}), 32.7$ (d, $J=8.4 \mathrm{~Hz}), 22.0$, 
20.3 ppm; ESI-MS: $m / z 277.0\left(\mathrm{M}^{+}+\mathrm{Na}\right)$; HRMS calcd for $\mathrm{C}_{12} \mathrm{H}_{16} \mathrm{O}_{4} \mathrm{P}[\mathrm{M}+\mathrm{H}]$ : 255.0781, found 255.0780 .

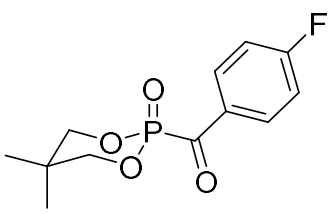

(5,5-Dimethyl-2-oxido-1,3,2-dioxaphosphinan-2-yl)(4-fluorophenyl)methanone

(5b). 96\% yield; White solid, m.p.: $119-121{ }^{\circ} \mathrm{C}$; IR (neat): $v\left(\mathrm{~cm}^{-1}\right) 3072,2972,1651$, $1595,1505,1474,1305,1280,1234,1160,1059,1012,985,850,770 ;{ }^{1} \mathrm{H}$ NMR (400 $\left.\mathrm{MHz}, \mathrm{CDCl}_{3}\right): \delta 8.39-8.45(\mathrm{~m}, 2 \mathrm{H}), 7.18(\mathrm{t}, J=8.8 \mathrm{~Hz}, 2 \mathrm{H}), 4.40(\mathrm{~d}, J=10.8 \mathrm{~Hz}, 2 \mathrm{H})$, 4.04 (dd, $J=19.2,10.8 \mathrm{~Hz}, 2 \mathrm{H}), 1.37$ (s, 3H), 0.89 (s, 3H) ppm; ${ }^{31} \mathrm{P}$ NMR (163 MHz, $\left.\mathrm{CDCl}_{3}\right): \delta-11.0 \mathrm{ppm} ;{ }^{19} \mathrm{~F}$ NMR $\left(376 \mathrm{MHz}, \mathrm{CDCl}_{3}\right): \delta-100.3 \mathrm{ppm} ;{ }^{13} \mathrm{C} \mathrm{NMR}(101 \mathrm{MHz}$, $\left.\mathrm{CDCl}_{3}\right): \delta 198.7(\mathrm{~d}, J=170.2 \mathrm{~Hz}), 167.0(\mathrm{~d}, J=260.8 \mathrm{~Hz}), 133.1(\mathrm{dd}, J=10.0,1.5 \mathrm{~Hz})$, $132.0(\mathrm{~d}, J=62.1 \mathrm{~Hz}), 116.3(\mathrm{dd}, J=22.2,1.6 \mathrm{~Hz}), 79.2(\mathrm{~d}, J=7.7 \mathrm{~Hz}), 32.8(\mathrm{~d}, J=$ 8.5 Hz), 22.0, 20.3 (d, $J=1.5 \mathrm{~Hz})$ ppm; ESI-MS: $m / z 294.9\left(\mathrm{M}^{+}+\mathrm{Na}\right)$; HRMS calcd for $\mathrm{C}_{12} \mathrm{H}_{15} \mathrm{FO}_{4} \mathrm{P}[\mathrm{M}+\mathrm{H}]: 273.0687$, found 273.0685 .

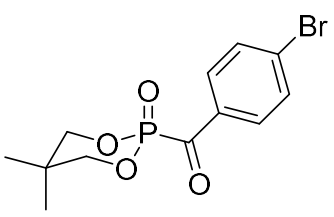

\section{(4-Bromophenyl)(5,5-dimethyl-2-oxido-1,3,2-dioxaphosphinan-2-yl)methanone}

(5c). $87 \%$ yield; White solid, m.p.: $98-100{ }^{\circ} \mathrm{C}$; IR (neat): $v\left(\mathrm{~cm}^{-1}\right) 3088,2969,1647$, 1580, 1561, 1473, 1309, 1281, 1224, 1059, 1011, 947, 842, 777; ${ }^{1} \mathrm{H}$ NMR (400 MHz, $\left.\mathrm{CDCl}_{3}\right): \delta 8.23(\mathrm{~d}, J=8.8 \mathrm{~Hz}, 2 \mathrm{H}), 7.66(\mathrm{~d}, J=8.8 \mathrm{~Hz}, 2 \mathrm{H}), 4.39(\mathrm{~d}, J=10.8 \mathrm{~Hz}, 2 \mathrm{H})$, $4.05(\mathrm{dd}, J=19.2,10.8 \mathrm{~Hz}, 2 \mathrm{H}), 1.38$ (s, 3H), 0.90 (s, 3H) ppm; ${ }^{31} \mathrm{P}$ NMR (163 MHz, $\left.\mathrm{CDCl}_{3}\right): \delta$-11.2 ppm; ${ }^{13} \mathrm{C} \mathrm{NMR}\left(101 \mathrm{MHz}, \mathrm{CDCl}_{3}\right): \delta 199.3$ (d, $\left.J=170.6 \mathrm{~Hz}\right), 133.7$ $(\mathrm{d}, J=63.8 \mathrm{~Hz}), 132.3,131.5(\mathrm{~d}, J=1.5 \mathrm{~Hz}), 131.1,79.2(\mathrm{~d}, J=8.4 \mathrm{~Hz}), 32.8(\mathrm{~d}, J=$ 8.4 Hz), 22.0, 20.3 (d, $J=1.5 \mathrm{~Hz})$ ppm; ESI-MS: $m / z 354.8\left(\mathrm{M}^{+}+\mathrm{Na}\right)$; HRMS calcd for $\mathrm{C}_{12} \mathrm{H}_{15} \mathrm{BrO}_{4} \mathrm{P}[\mathrm{M}+\mathrm{H}]:$ 332.9886, found 332.9885 . 


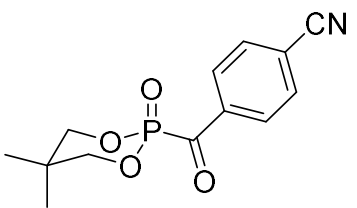

4-(5,5-Dimethyl-2-oxido-1,3,2-dioxaphosphinane-2-carbonyl)benzonitrile (5d). 91\% yield; White solid, m.p.: $104-106^{\circ} \mathrm{C}$; IR (neat): $v\left(\mathrm{~cm}^{-1}\right)$ 3095, 2971, 2231, 1658, 1603 , 1560, 1474, 1375, 1284, 1223, 1059, 1013, 985, 852, 757; ${ }^{1} \mathrm{H} \mathrm{NMR}\left(400 \mathrm{MHz}, \mathrm{CDCl}_{3}\right)$ : $\delta 8.45(\mathrm{~d}, J=8.4 \mathrm{~Hz}, 2 \mathrm{H}), 7.80(\mathrm{~d}, J=7.6 \mathrm{~Hz}, 2 \mathrm{H}), 4.38(\mathrm{~d}, J=10.8 \mathrm{~Hz}, 2 \mathrm{H}), 4.07(\mathrm{dd}$, $J=18.8,11.2 \mathrm{~Hz}, 2 \mathrm{H}), 1.37$ (s, 3H), 0.90 (s, 3H) ppm; ${ }^{31} \mathrm{P} \mathrm{NMR}\left(163 \mathrm{MHz}, \mathrm{CDCl}_{3}\right): \delta$ -12.2 ppm; ${ }^{13} \mathrm{C}$ NMR (101 MHz, $\left.\mathrm{CDCl}_{3}\right): \delta 200.1$ (d, $\left.J=172.6 \mathrm{~Hz}\right), 137.8(\mathrm{~d}, J=63.6$ Hz), 132.6 (d, $J=1.6 \mathrm{~Hz}), 130.3,118.0,117.6,79.4$ (d, $J=7.7 \mathrm{~Hz}), 32.8$ (d, $J=8.4$ Hz), $21.8(\mathrm{~d}, J=12.2 \mathrm{~Hz}), 20.3(\mathrm{~d}, J=1.6 \mathrm{~Hz}) \mathrm{ppm}$; ESI-MS: $m / z 302.0\left(\mathrm{M}^{+}+\mathrm{Na}\right)$; HRMS calcd for $\mathrm{C}_{13} \mathrm{H}_{15} \mathrm{NO}_{4} \mathrm{P}[\mathrm{M}+\mathrm{H}]: 280.0733$, found 280.0732 .

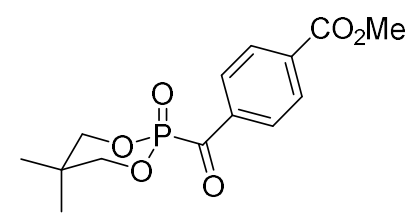

Methyl 4-(5,5-dimethyl-2-oxido-1,3,2-dioxaphosphinane-2-carbonyl)benzoate (5e). 87\% yield; White solid, m.p.: $98-100{ }^{\circ} \mathrm{C}$; IR (neat): $v\left(\mathrm{~cm}^{-1}\right)$ 2969, 1726, 1651, 1607, 1474, 1437, 1407, 1282, 1109, 1059, 1013, 985, 834, 775; ${ }^{1} \mathrm{H}$ NMR (400 MHz, $\left.\mathrm{CDCl}_{3}\right)$ : $\delta 8.40(\mathrm{dd}, J=8.0,1.6 \mathrm{~Hz}, 2 \mathrm{H}), 8.15(\mathrm{~d}, J=8.0 \mathrm{~Hz}, 2 \mathrm{H}), 4.38(\mathrm{~d}, J=11.2 \mathrm{~Hz}, 2 \mathrm{H})$, $4.06(\mathrm{dd}, J=19.2,10.8 \mathrm{~Hz}, 2 \mathrm{H}), 3.95(\mathrm{~d}, J=2.0 \mathrm{~Hz}, 3 \mathrm{H}), 1.38(\mathrm{~s}, 3 \mathrm{H}), 0.90(\mathrm{~s}, 3 \mathrm{H})$ ppm; ${ }^{31} \mathrm{P} \mathrm{NMR}\left(163 \mathrm{MHz}, \mathrm{CDCl}_{3}\right): \delta-11.3 \mathrm{ppm} ;{ }^{13} \mathrm{C} \mathrm{NMR}\left(101 \mathrm{MHz}, \mathrm{CDCl}_{3}\right): \delta 200.4$ (d, $J=170.2 \mathrm{~Hz}), 165.9,138.1(\mathrm{~d}, J=62.9 \mathrm{~Hz}), 135.4,130.0,129.9$ (d, $J=1.5 \mathrm{~Hz})$, $79.2(\mathrm{~d}, J=7.7 \mathrm{~Hz}), 52.6,32.8(\mathrm{~d}, J=8.4 \mathrm{~Hz}), 22.0,20.3(\mathrm{~d}, J=1.5 \mathrm{~Hz}) \mathrm{ppm}$; ESI-MS: $m / z 335.0\left(\mathrm{M}^{+}+\mathrm{Na}\right)$; HRMS calcd for $\mathrm{C}_{14} \mathrm{H}_{18} \mathrm{O}_{6} \mathrm{P}[\mathrm{M}+\mathrm{H}]:$ : 313.0836, found 313.0833.

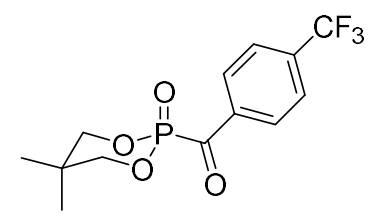

(5,5-Dimethyl-2-oxido-1,3,2-dioxaphosphinan-2-yl)(4-(trifluoromethyl)phenyl)methanone (5f). 93\% yield; White solid, m.p.: $77-79^{\circ} \mathrm{C}$; IR (neat): $v\left(\mathrm{~cm}^{-1}\right) 3080,2976$, 
1649, 1503, 1474, 1412, 1325, 1281, 1170, 1062, 1016, 861, 763; ${ }^{1} \mathrm{H}$ NMR (400 MHz, $\left.\mathrm{CDCl}_{3}\right): \delta 8.47(\mathrm{~d}, J=8.4 \mathrm{~Hz}, 2 \mathrm{H}), 7.77(\mathrm{~d}, J=8.4 \mathrm{~Hz}, 2 \mathrm{H}), 4.38(\mathrm{~d}, J=10.8 \mathrm{~Hz}, 2 \mathrm{H})$, 4.07 (dd, $J=18.8,10.8 \mathrm{~Hz}, 2 \mathrm{H}), 1.38$ (s, 3H), 0.90 (s, 3H) ppm; ${ }^{31} \mathrm{P}$ NMR (163 MHz, $\mathrm{CDCl}_{3}$ ): $\delta$-11.6 ppm; ${ }^{19} \mathrm{~F}$ NMR (376 MHz, $\left.\mathrm{CDCl}_{3}\right): \delta-63.5 \mathrm{ppm} ;{ }^{13} \mathrm{C} \mathrm{NMR}(101 \mathrm{MHz}$, $\mathrm{CDCl}_{3}$ ): $\delta 200.2(\mathrm{~d}, J=171.0 \mathrm{~Hz}), 137.7$ (d, $\left.J=62.1 \mathrm{~Hz}\right), 136.0(\mathrm{q}, J=33.2 \mathrm{~Hz}), 130.4$ (d, $J=1.5 \mathrm{~Hz}), 125.9$ (q, $J=3.7 \mathrm{~Hz}), 123.3$ (q, $J=274.5 \mathrm{~Hz}), 79.3(\mathrm{~d}, J=7.7 \mathrm{~Hz}), 32.8$ (d, $J=8.4 \mathrm{~Hz}), 21.9,20.3$ (d, $J=1.5 \mathrm{~Hz})$ ppm; ESI-MS: $m / z 344.9\left(\mathrm{M}^{+}+\mathrm{Na}\right)$; HRMS calcd for $\mathrm{C}_{13} \mathrm{H}_{15} \mathrm{~F}_{3} \mathrm{O}_{4} \mathrm{P}[\mathrm{M}+\mathrm{H}]:$ 323.0655, found 323.0653.

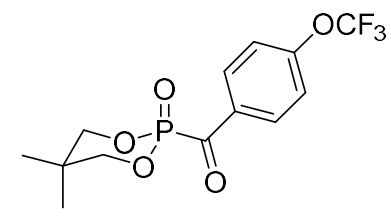

\section{(5,5-Dimethyl-2-oxido-1,3,2-dioxaphosphinan-2-yl)(4-(trifluoromethoxy)phenyl)-} methanone (5g). 90\% yield; White solid, m.p.: 56-58 ${ }^{\circ} \mathrm{C}$; IR (neat): $v\left(\mathrm{~cm}^{-1}\right) 3078$, $2973,1654,1599,1505,1474,1375,1262,1224,1172,1061,1014,948,836,774 ;{ }^{1} \mathrm{H}$ NMR (400 MHz, $\left.\mathrm{CDCl}_{3}\right): \delta 8.43(\mathrm{dd}, J=8.8,1.6 \mathrm{~Hz}, 2 \mathrm{H}), 7.31(\mathrm{~d}, J=8.8 \mathrm{~Hz}, 2 \mathrm{H})$, 4.39 (d, $J=11.2 \mathrm{~Hz}, 2 \mathrm{H}), 4.04$ (dd, $J=18.4,10.0 \mathrm{~Hz}, 2 \mathrm{H}), 1.37$ (s, 3H), 0.89 (s, 3H) ppm; ${ }^{31} \mathrm{P} \mathrm{NMR}\left(163 \mathrm{MHz}, \mathrm{CDCl}_{3}\right): \delta-11.3 \mathrm{ppm} ;{ }^{19} \mathrm{~F} \mathrm{NMR}\left(376 \mathrm{MHz}, \mathrm{CDCl}_{3}\right): \delta-57.6$ ppm; ${ }^{13} \mathrm{C}$ NMR (101 MHz, $\left.\mathrm{CDCl}_{3}\right): \delta 199.1$ (d, $\left.J=171.0 \mathrm{~Hz}\right), 154.0$ (q, $J=1.5 \mathrm{~Hz}$ ), $133.4(\mathrm{~d}, J=64.4 \mathrm{~Hz}), 132.3(\mathrm{~d}, J=1.5 \mathrm{~Hz}), 120.4(\mathrm{~d}, J=1.5 \mathrm{~Hz}), 120.1(\mathrm{q}, J=260.7$ Hz), 79.3 (d, $J=7.6 \mathrm{~Hz}), 32.8$ (d, $J=8.5 \mathrm{~Hz}), 21.9,20.3$ ppm; ESI-MS: $m / z 360.9$ $\left(\mathrm{M}^{+}+\mathrm{Na}\right)$; HRMS calcd for $\mathrm{C}_{13} \mathrm{H}_{15} \mathrm{~F}_{3} \mathrm{O}_{5} \mathrm{P}[\mathrm{M}+\mathrm{H}]: 339.0604$, found 339.0602.

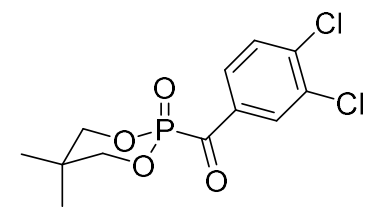

\section{(3,4-Dichlorophenyl)(5,5-dimethyl-2-oxido-1,3,2-dioxaphosphinan-2-}

yl)methanone (5h). 90\% yield; White solid, m.p.: 112-114 ${ }^{\circ} \mathrm{C}$; IR (neat): $v\left(\mathrm{~cm}^{-1}\right) 3071$, 2968, 2926, 1654, 1579, 1465, 1376, 1287, 1212, 1060, 1014, 985, 839, 749; ${ }^{1} \mathrm{H}$ NMR $\left(400 \mathrm{MHz}, \mathrm{CDCl}_{3}\right): \delta 8.39(\mathrm{~s}, 1 \mathrm{H}), 8.27(\mathrm{~d}, J=8.8 \mathrm{~Hz}, 1 \mathrm{H}), 7.60(\mathrm{~d}, J=8.8 \mathrm{~Hz}, 1 \mathrm{H})$, 
$4.38(\mathrm{~d}, J=10.8 \mathrm{~Hz}, 2 \mathrm{H}), 4.06(\mathrm{dd}, J=19.2,10.4 \mathrm{~Hz}, 2 \mathrm{H}), 1.38(\mathrm{~s}, 3 \mathrm{H}), 0.90(\mathrm{~s}, 3 \mathrm{H})$ ppm; ${ }^{31} \mathrm{P} \mathrm{NMR}\left(163 \mathrm{MHz}, \mathrm{CDCl}_{3}\right): \delta-11.8 \mathrm{ppm} ;{ }^{13} \mathrm{C} \mathrm{NMR}\left(101 \mathrm{MHz}, \mathrm{CDCl}_{3}\right): \delta 198.8$ $(\mathrm{d}, J=173.3 \mathrm{~Hz}), 140.1,134.8(\mathrm{~d}, J=65.1 \mathrm{~Hz}), 133.8(\mathrm{~d}, J=2.2 \mathrm{~Hz}), 131.4(\mathrm{~d}, J=2.3$ Hz), 131.1, 134.4 (d, $J=9.2 \mathrm{~Hz}), 79.4$ (d, $J=7.7 \mathrm{~Hz}), 32.8$ (d, $J=8.5 \mathrm{~Hz}), 22.0,20.3$ $(\mathrm{d}, J=1.5 \mathrm{~Hz})$ ppm; ESI-MS: $m / z 322.8\left(\mathrm{M}^{+}+\mathrm{H}\right)$; HRMS calcd for $\mathrm{C}_{12} \mathrm{H}_{14} \mathrm{Cl}_{2} \mathrm{O}_{4} \mathrm{P}$ $[\mathrm{M}+\mathrm{H}]:$ 323.0001, found 322.9999.

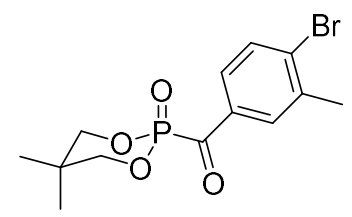

(3-Bromo-4-methylphenyl)(5,5-dimethyl-2-oxido-1,3,2-dioxaphosphinan-2-

yl)methanone (5i). 94\% yield; White solid, m.p.: 75-77 ${ }^{\circ} \mathrm{C}$; IR (neat): $v\left(\mathrm{~cm}^{-1}\right.$ ) 3061, 2971, 1652, 1593, 1552, 1474, 1375, 1290, 1223, 1060, 987, 838, 767; ${ }^{1} \mathrm{H}$ NMR (400 $\left.\mathrm{MHz}, \mathrm{CDCl}_{3}\right): \delta 8.41(\mathrm{~s}, 1 \mathrm{H}), 8.29(\mathrm{dd}, J=8.0,1.2 \mathrm{~Hz}, 1 \mathrm{H}), 7.37(\mathrm{~d}, J=8.0 \mathrm{~Hz}, 1 \mathrm{H})$, $4.37(\mathrm{~d}, J=10.8 \mathrm{~Hz}, 2 \mathrm{H}), 4.04(\mathrm{dd}, J=18.8,10.8 \mathrm{~Hz}, 2 \mathrm{H}), 2.47(\mathrm{~s}, 3 \mathrm{H}), 1.37$ (s, 3H), 0.89 (s, 3H) ppm; ${ }^{31} \mathrm{P}$ NMR (163 MHz, $\left.\mathrm{CDCl}_{3}\right): \delta-11.0 \mathrm{ppm} ;{ }^{13} \mathrm{C}$ NMR $(101 \mathrm{MHz}$, $\left.\mathrm{CDCl}_{3}\right): \delta 198.8(\mathrm{~d}, J=171.0 \mathrm{~Hz}), 146.2,134.7(\mathrm{~d}, J=64.4 \mathrm{~Hz}), 133.2(\mathrm{~d}, J=3.0 \mathrm{~Hz})$, 131.2, 129.4, 125.6 (d, $J=3.1 \mathrm{~Hz}), 79.1$ (d, $J=8.5 \mathrm{~Hz}), 32.7$ (d, $J=8.4 \mathrm{~Hz}), 23.5,22.0$, $20.3(\mathrm{~d}, J=1.6 \mathrm{~Hz})$ ppm; ESI-MS: $m / z$ 368.6/370.6 $\left(\mathrm{M}^{+}+\mathrm{Na}\right)$; HRMS calcd for $\mathrm{C}_{13} \mathrm{H}_{17} \mathrm{BrO}_{4} \mathrm{P}[\mathrm{M}+\mathrm{H}]:$ 347.0042, found 347.0041.

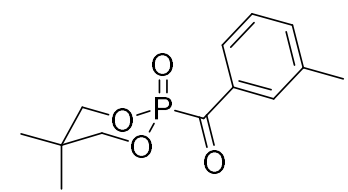

(5,5-Dimethyl-2-oxido-1,3,2-dioxaphosphinan-2-yl)(m-tolyl)methanone (5j). $90 \%$ yield; White solid, m.p.: $84-86{ }^{\circ} \mathrm{C}$; IR (neat): $v\left(\mathrm{~cm}^{-1}\right)$ 3072, 2970, 1649, 1599, 1582 , $1473,1374,1280,1257,1061,1010,835,763 ;{ }^{1} \mathrm{H}$ NMR (400 MHz, $\left.\mathrm{CDCl}_{3}\right): \delta 8.17(\mathrm{~d}$, $J=7.2 \mathrm{~Hz}, 1 \mathrm{H}), 8.13(\mathrm{~s}, 1 \mathrm{H}), 7.47(\mathrm{~d}, J=7.2 \mathrm{~Hz}, 1 \mathrm{H}), 7.40$ (t, $J=7.2 \mathrm{~Hz}, 1 \mathrm{H}), 4.37$ (d, $J=10.8 \mathrm{~Hz}, 2 \mathrm{H}), 4.04$ (dd, $J=18.8,10.4 \mathrm{~Hz}, 2 \mathrm{H}), 2.43$ (s, 3H), 1.38 (s, 3H), 0.89 (s, 3H) ppm; ${ }^{31} \mathrm{P}$ NMR (163 MHz, $\left.\mathrm{CDCl}_{3}\right): \delta-10.0 \mathrm{ppm} ;{ }^{13} \mathrm{C} \mathrm{NMR}\left(101 \mathrm{MHz}, \mathrm{CDCl}_{3}\right): \delta$ 
$200.2(\mathrm{~d}, J=167.2 \mathrm{~Hz}), 138.8,136.1,135.3(\mathrm{~d}, J=62.8 \mathrm{~Hz}), 130.2(\mathrm{~d}, J=2.3 \mathrm{~Hz})$, $128.8(\mathrm{~d}, J=1.5 \mathrm{~Hz}), 127.4(\mathrm{~d}, J=1.5 \mathrm{~Hz}), 79.0(\mathrm{~d}, J=7.7 \mathrm{~Hz}), 32.7(\mathrm{~d}, J=8.5 \mathrm{~Hz})$, 22.0, 21.3, 20.3 (d, $J=1.6 \mathrm{~Hz})$ ppm; ESI-MS: $m / z 290.9\left(\mathrm{M}^{+}+\mathrm{Na}\right)$; HRMS calcd for $\mathrm{C}_{13} \mathrm{H}_{18} \mathrm{O}_{4} \mathrm{P}[\mathrm{M}+\mathrm{H}]:$ 269.0937, found 269.0937.

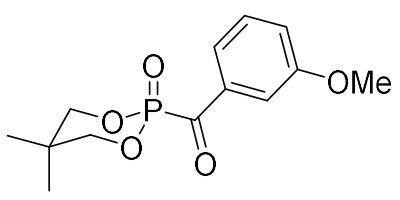

\section{(5,5-Dimethyl-2-oxido-1,3,2-dioxaphosphinan-2-yl)(3-methoxyphenyl)methanone}

(5k). 88\% yield; White solid, m.p.: 92-94 ${ }^{\circ} \mathrm{C}$; IR (neat): $v\left(\mathrm{~cm}^{-1}\right)$ 3074, 2969, 1651, $1595,1579,1485,1374,1280,1174,1060,984,834,761 ;{ }^{1} \mathrm{H} \mathrm{NMR}\left(400 \mathrm{MHz}, \mathrm{CDCl}_{3}\right)$ : $\delta 8.04(\mathrm{~d}, J=7.6 \mathrm{~Hz}, 1 \mathrm{H}), 7.76(\mathrm{~s}, 1 \mathrm{H}), 7.41$ (t, $J=7.6 \mathrm{~Hz}, 1 \mathrm{H}), 7.19$ (d, $J=7.6,1 \mathrm{H})$, $4.37(\mathrm{~d}, J=10.4 \mathrm{~Hz}, 2 \mathrm{H}), 4.03(\mathrm{dd}, J=18.8,10.8 \mathrm{~Hz}, 2 \mathrm{H}), 3.85(\mathrm{~s}, 3 \mathrm{H}), 1.37$ (s, 3H), $0.88(\mathrm{~s}, 3 \mathrm{H}) \mathrm{ppm} ;{ }^{31} \mathrm{P}$ NMR (163 MHz, $\left.\mathrm{CDCl}_{3}\right): \delta-10.4 \mathrm{ppm} ;{ }^{13} \mathrm{C}$ NMR $(101 \mathrm{MHz}$, $\left.\mathrm{CDCl}_{3}\right): \delta 200.0(\mathrm{~d}, J=168.7 \mathrm{~Hz}), 159.9(\mathrm{~d}, J=2.2 \mathrm{~Hz}), 136.5(\mathrm{~d}, J=63.6 \mathrm{~Hz}), 130.0$, 123.5, 122.6, 112.7 (d, $J=3.1 \mathrm{~Hz}), 79.1$ (d, $J=7.7 \mathrm{~Hz}), 55.4,32.7$ (d, $J=7.7 \mathrm{~Hz}), 22.0$, $20.3(\mathrm{~d}, J=1.5 \mathrm{~Hz})$ ppm; ESI-MS: $m / z 306.9\left(\mathrm{M}^{+}+\mathrm{Na}\right)$; HRMS calcd for $\mathrm{C}_{13} \mathrm{H}_{18} \mathrm{O}_{5} \mathrm{P}$ $[\mathrm{M}+\mathrm{H}]: 285.0886$, found 285.0885 .

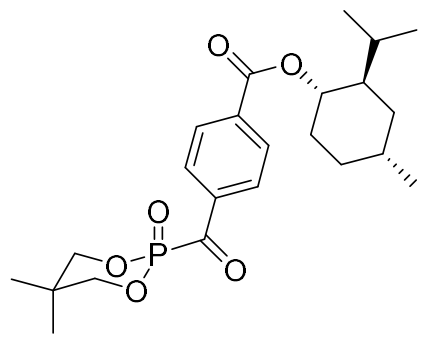

$(1 S, 2 R, 4 R)-2$-isopropyl-4-methylcyclohexyl

4-(5,5-dimethyl-2-oxido-1,3,2dioxaphosphinane-2-carbonyl)benzoate (51). 89\% yield; white foam; IR (neat): $v$ $\left(\mathrm{cm}^{-1}\right)$ 2957, 2873, 1717, 1652, 1607, 1567, 1467, 1373, 1276, 1117, 1062, 984, 834, 733; ${ }^{1} \mathrm{H}$ NMR (400 MHz, $\left.\mathrm{CDCl}_{3}\right): \delta 8.39(\mathrm{~d}, J=8.0 \mathrm{~Hz}, 2 \mathrm{H}), 8.14(\mathrm{~d}, J=8.0 \mathrm{~Hz}, 2 \mathrm{H})$, $4.93(\mathrm{td}, J=10.8,4.0 \mathrm{~Hz}, 1 \mathrm{H}), 4.36(\mathrm{~d}, J=10.8 \mathrm{~Hz}, 2 \mathrm{H}), 4.04(\mathrm{dd}, J=18.8,10.8 \mathrm{~Hz}$, 2H), 2.06-2.15 (m, 1H), 1.84-1.96 (m, 1H), 1.67-1.76 (m, 2H), 1.48-1.61 (m, 2H), 
$1.37(\mathrm{~s}, 3 \mathrm{H}), 1.03-1.18(\mathrm{~m}, 2 \mathrm{H}), 0.85-0.95(\mathrm{~m}, 10 \mathrm{H}), 0.77(\mathrm{~d}, J=7.2 \mathrm{~Hz}, 3 \mathrm{H}) \mathrm{ppm} ;{ }^{31} \mathrm{P}$ NMR (163 MHz, $\left.\left.\mathrm{CDCl}_{3}\right): \delta-11.2 \mathrm{ppm} ;{ }^{13} \mathrm{C} \mathrm{NMR} \mathrm{(101} \mathrm{MHz,} \mathrm{CDCl}_{3}\right): \delta 200.4(\mathrm{~d}, J=$ $170.3 \mathrm{~Hz}), 164.9,138.0$ (d, $J=62.8 \mathrm{~Hz}), 136.1,130.0,129.9$ (d, $J=1.5 \mathrm{~Hz}), 79.2$ (dd, $J=7.7,1.5 \mathrm{~Hz}), 75.7,47.1,40.8,34.1,32.7$ (d, $J=8.5 \mathrm{~Hz}), 31.4,26.5,23.6,21.9,20.7$, 20.3, 16.5 ppm; ESI-MS: m/z $459.0\left(\mathrm{M}^{+}+\mathrm{Na}\right)$; HRMS calcd for $\mathrm{C}_{23} \mathrm{H}_{34} \mathrm{O}_{6} \mathrm{P}[\mathrm{M}+\mathrm{H}]$ : 437.2088, found 437.2087.

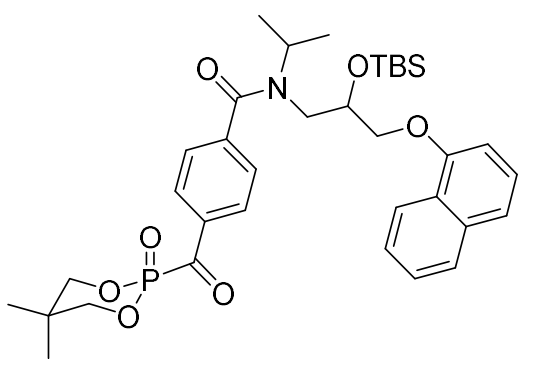

\section{$N$-(2-((tert-Butyldimethylsilyl)oxy)-3-(naphthalen-1-yloxy)propyl)-4-(5,5-}

\section{dimethyl-2-oxido-1,3,2-dioxaphosphinane-2-carbonyl)- $N$-isopropylbenzamide}

(5m). 91\% yield; white foam; IR (neat): $v\left(\mathrm{~cm}^{-1}\right)$ 3054, 2958, 2930, 1637, 1604, 1581, 1463, 1404, 1283, 1112, 1061, 836, 774, 733; ${ }^{1} \mathrm{H}$ NMR (400 MHz, $\left.\mathrm{CDCl}_{3}\right): \delta 8.21-8.50$ (m, 3H), 7.79 (d, $J=5.2 \mathrm{~Hz}, 1 \mathrm{H}), 7.40-7.60(\mathrm{~m}, 5 \mathrm{H}), 7.36$ (t, $J=8.0 \mathrm{~Hz}, 1 \mathrm{H}), 6.83(\mathrm{~d}$, $J=7.2 \mathrm{~Hz}, 1 \mathrm{H}), 4.76-4.87(\mathrm{~m}, 1 \mathrm{H}), 4.37$ (d, $J=10.8 \mathrm{~Hz}, 2 \mathrm{H}), 4.11-4.27$ (m, 2H), 4.04 $(\mathrm{dd}, J=19.2,10.8 \mathrm{~Hz}, 2 \mathrm{H}), 3.86-3.97(\mathrm{~m}, 1 \mathrm{H}), 3.79(\mathrm{dd}, J=13.2,6.0 \mathrm{~Hz}, 1 \mathrm{H}), 3.45$ $(\mathrm{dd}, J=13.2,6.4 \mathrm{~Hz}, 1 \mathrm{H}), 1.37$ (s, 3H), $1.23(\mathrm{~d}, J=6.0 \mathrm{~Hz}, 3 \mathrm{H}), 1.10$ (d, $J=6.0 \mathrm{~Hz}$, $3 \mathrm{H}), 0.93$ (s, 9H), 0.87 (s, 3H), 0.21 (s, 3H), 0.15 (s, 3H) ppm; ${ }^{31} \mathrm{P}$ NMR (163 MHz, $\left.\mathrm{CDCl}_{3}\right): \delta-10.8 \mathrm{ppm} ;{ }^{13} \mathrm{C} \mathrm{NMR}\left(101 \mathrm{MHz}, \mathrm{CDCl}_{3}\right): \delta 199.8(\mathrm{~d}, J=169.4 \mathrm{~Hz})$, 171.4/170.0, 154.5/152.0, 143.4/139.5, 135.6 (d, $J=62.8 \mathrm{~Hz}), 134.4,130.3,127.4$, 126.8, 126.3, 125.8, 125.5, 125.1, 122.0, 120.4, 104.6, 79.1 (d, $J=7.7 \mathrm{~Hz}), 71.1,68.3$, 50.8, 45.0, 32.7 (d, $J=8.4 \mathrm{~Hz}), 25.8,21.9,21.4,20.9,20.2,18.0,-4.3,-4.6$ ppm; ESIMS: $m / z 654.2\left(\mathrm{M}^{+}+\mathrm{H}\right)$; HRMS calcd for $\mathrm{C}_{35} \mathrm{H}_{49} \mathrm{NO}_{7} \mathrm{PSi}[\mathrm{M}+\mathrm{H}]: 654.3010$, found 654.3007 . 


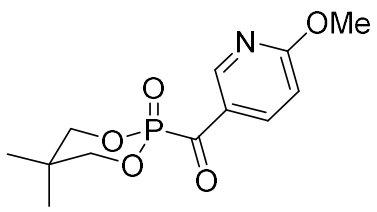

(5,5-Dimethyl-2-oxido-1,3,2-dioxaphosphinan-2-yl)(6-methoxypyridin-3-

yl)methanone (5n). $88 \%$ yield; White solid, m.p.: $111-113^{\circ} \mathrm{C}$; IR (neat): $v\left(\mathrm{~cm}^{-1}\right) 2968$, 1643, 1598, 1557, 1495, 1378, 1282, 1244, 1059, 1011, 946, 839, 770; ${ }^{1} \mathrm{H}$ NMR (400 $\left.\mathrm{MHz}, \mathrm{CDCl}_{3}\right): \delta 9.25-9.30(\mathrm{~m}, 1 \mathrm{H}), 8.38-8.45(\mathrm{~m}, 1 \mathrm{H}), 6.77-6.83(\mathrm{~m}, 1 \mathrm{H}), 4.41(\mathrm{~d}, J$ $=10.4 \mathrm{~Hz}, 2 \mathrm{H}), 3.98-4.09(\mathrm{~m}, 5 \mathrm{H}), 1.37$ (d, $J=3.2 \mathrm{~Hz}, 3 \mathrm{H}), 0.89(\mathrm{~d}, J=3.2 \mathrm{~Hz}, 3 \mathrm{H})$ ppm; ${ }^{31} \mathrm{P}$ NMR (163 MHz, $\left.\mathrm{CDCl}_{3}\right): \delta-11.3 \mathrm{ppm} ;{ }^{13} \mathrm{C} \mathrm{NMR}\left(101 \mathrm{MHz}, \mathrm{CDCl}_{3}\right): \delta$ $198.2(\mathrm{~d}, J=171.8 \mathrm{~Hz}), 167.7,152.5,139.1(\mathrm{~d}, J=2.2 \mathrm{~Hz}), 126.0(\mathrm{~d}, J=65.2 \mathrm{~Hz})$, 111.5, $79.2(\mathrm{~d}, J=7.7 \mathrm{~Hz}), 54.4,32.8(\mathrm{~d}, J=8.5 \mathrm{~Hz}), 22.0,20.3(\mathrm{~d}, J=1.5 \mathrm{~Hz}) \mathrm{ppm}$; ESI-MS: $m / z 285.9\left(\mathrm{M}^{+}+\mathrm{H}\right)$; HRMS calcd for $\mathrm{C}_{12} \mathrm{H}_{17} \mathrm{NO}_{5} \mathrm{P}[\mathrm{M}+\mathrm{H}]: 286.0839$, found 286.0838 .

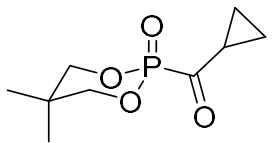

Cyclopropyl(5,5-dimethyl-2-oxido-1,3,2-dioxaphosphinan-2-yl)methanone (5o). 83\% yield; colorless oil ; IR (neat): $v\left(\mathrm{~cm}^{-1}\right)$ 3019, 2972, 1669, 1474, 1374, 1285, 1072 , 1049, 999, 834, 777; ${ }^{1} \mathrm{H}$ NMR (400 MHz, $\left.\mathrm{CDCl}_{3}\right): \delta 4.28$ (d, $\left.J=10.8 \mathrm{~Hz}, 2 \mathrm{H}\right), 3.95$ $(\mathrm{dd}, J=18.8,10.8 \mathrm{~Hz}, 2 \mathrm{H}), 2.67-2.75(\mathrm{~m}, 1 \mathrm{H}), 1.25-1.31(\mathrm{~m}, 5 \mathrm{H}), 1.11-1.17(\mathrm{~m}, 2 \mathrm{H})$, 0.83 (s, 3H) ppm; ${ }^{31} \mathrm{P}$ NMR (163 MHz, $\left.\mathrm{CDCl}_{3}\right): \delta-11.7 \mathrm{ppm} ;{ }^{13} \mathrm{C}$ NMR $(101 \mathrm{MHz}$, $\left.\mathrm{CDCl}_{3}\right): \delta 212.7(\mathrm{~d}, J=167.2 \mathrm{~Hz}), 78.9(\mathrm{~d}, J=6.9 \mathrm{~Hz}), 32.6(\mathrm{~d}, J=8.5 \mathrm{~Hz}), 23.2,22.5$, 21.8, 20.2, 14.3 ppm; ESI-MS: m/z $240.9\left(\mathrm{M}^{+}+\mathrm{Na}\right)$; HRMS calcd for $\mathrm{C}_{9} \mathrm{H}_{16} \mathrm{O}_{4} \mathrm{P}[\mathrm{M}+\mathrm{H}]$ : 219.0781 , found 219.0781 .

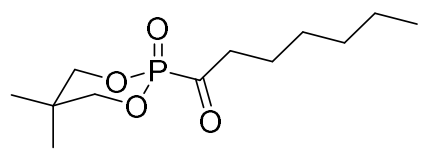

1-(5,5-Dimethyl-2-oxido-1,3,2-dioxaphosphinan-2-yl)heptan-1-one (5p). 94\% yield; White solid, m.p.: $26-28{ }^{\circ} \mathrm{C}$; IR (neat): $v\left(\mathrm{~cm}^{-1}\right)$ 2959, 2932, 2873, 2857, 1690, 1468, 1286, 1066, 1009, 986, 834, 789; ${ }^{1} \mathrm{H}$ NMR (400 MHz, $\left.\mathrm{CDCl}_{3}\right): \delta 4.28$ (d, $J=11.2 \mathrm{~Hz}$, 
2H), $3.97(\mathrm{dd}, J=18.4,11.2 \mathrm{~Hz}, 2 \mathrm{H}), 2.87(\mathrm{t}, J=7.6 \mathrm{~Hz}, 2 \mathrm{H}), 1.54-1.65(\mathrm{~m}, 2 \mathrm{H})$, $1.18-1.32(\mathrm{~m}, 9 \mathrm{H}), 0.80-0.86(\mathrm{~m}, 6 \mathrm{H}) \mathrm{ppm} ;{ }^{31} \mathrm{P} \mathrm{NMR}$ (163 MHz, $\left.\mathrm{CDCl}_{3}\right): \delta-12.0 \mathrm{ppm}$; ${ }^{13} \mathrm{C} \mathrm{NMR}\left(101 \mathrm{MHz}, \mathrm{CDCl}_{3}\right): \delta 213.9(\mathrm{~d}, J=156.4 \mathrm{~Hz}), 78.9(\mathrm{~d}, J=7.7 \mathrm{~Hz}), 44.1(\mathrm{~d}$, $J=53.6 \mathrm{~Hz}), 32.6(\mathrm{~d}, J=8.4 \mathrm{~Hz}), 31.3,28.4,22.3,22.1(\mathrm{~d}, J=3.8 \mathrm{~Hz}), 21.7,20.3(\mathrm{~d}$, $J=1.6 \mathrm{~Hz}), 13.9(\mathrm{~d}, J=2.3 \mathrm{~Hz})$ ppm; ESI-MS: $m / z 285.0\left(\mathrm{M}^{+}+\mathrm{Na}\right)$; HRMS calcd for $\mathrm{C}_{12} \mathrm{H}_{24} \mathrm{O}_{4} \mathrm{P}[\mathrm{M}+\mathrm{H}]: 263.1407$, found 263.1406.

\section{Procedure for the preparation of $3 i$}

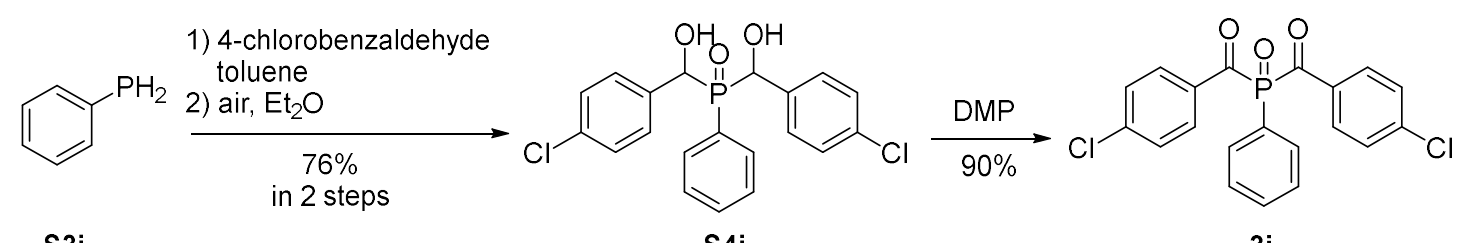

S3i

S4i

$3 \mathbf{i}$

To a solution of 4-chlorobenzaldehyde $(700 \mathrm{mg}, 5.0 \mathrm{mmol})$ in toluene $(10 \mathrm{~mL})$ was added phenylphosphane $\mathbf{S 3 i ^ { 2 6 }}$ (220 mg, $2 \mathrm{mmol}$ ) at room temperature under argon atmosphere. The solution was stirred at room temperature for $12 \mathrm{~h}$ and then concentrated under reduced pressure. $\mathrm{Et}_{2} \mathrm{O}(20 \mathrm{~mL})$ was added into the residue and the mixture was stirred at room temperature under air for another $12 \mathrm{~h}$. The precipitate was filtered and washed with $\mathrm{Et}_{2} \mathrm{O}$. Alcohol $\mathbf{S 4 i}$ was thus obtained as a white solid. Yield: $618 \mathrm{mg}(76 \%)$.

To a solution of $\mathbf{S 4 i}$ (406 mg, $1.0 \mathrm{mmol})$ in $\mathrm{CH}_{2} \mathrm{Cl}_{2}(4 \mathrm{~mL})$ was added DMP (638 mg, $1.5 \mathrm{mmol}$ ) in 3 portions and the mixture was stirred at $\mathrm{rt}$ for $2 \mathrm{~h}$. The reaction was quenched with saturated aqueous $\mathrm{Na}_{2} \mathrm{SO}_{3}(10 \mathrm{~mL})$ and the resulting mixture was extracted with $\mathrm{CH}_{2} \mathrm{Cl}_{2}(3 \times 10 \mathrm{~mL})$. The combined organic layers were washed successively with saturated aqueous $\mathrm{Na}_{2} \mathrm{SO}_{3}(4 \times 20 \mathrm{~mL})$, saturated aqueous $\mathrm{NaHCO}_{3}$ $(4 \times 20 \mathrm{~mL})$ and brine $(20 \mathrm{~mL})$, dried over anhydrous $\mathrm{Na}_{2} \mathrm{SO}_{4}$, filtered, and concentrated under reduced pressure. Ketone 3i was thus obtained as a light yellow oil directly used without further purification. Yield: $364 \mathrm{mg}(90 \%)$. 
<smiles>O=C(c1ccc(Cl)cc1)P(C(=O)c1ccc(Cl)cc1)c1ccccc1</smiles>

(Phenylphosphoryl)bis((4-chlorophenyl)methanone) (3i). Unstable; Light yellow oil; IR (neat): $v\left(\mathrm{~cm}^{-1}\right)$ 3095, 3064, 2963, 2924, 2854, 1667, 1641, 1583, 1486, 1402, 1220 , 1176, 1090, 1014, 837, $737{ }^{1} \mathrm{H}$ NMR (400 MHz, $\left.\mathrm{CDCl}_{3}\right): \delta 8.31(\mathrm{~d}, J=8.0 \mathrm{~Hz}, 4 \mathrm{H})$, $7.94(\mathrm{t}, J=8.4 \mathrm{~Hz}, 2 \mathrm{H}), 7.65(\mathrm{t}, J=7.2 \mathrm{~Hz}, 1 \mathrm{H}), 7.52-7.60(\mathrm{~m}, 2 \mathrm{H}), 7.47(\mathrm{~d}, J=8.0$ $\mathrm{Hz}, 4 \mathrm{H}) \mathrm{ppm} ;{ }^{31} \mathrm{P}$ NMR (163 MHz, $\left.\left.\mathrm{CDCl}_{3}\right): \delta 19.7 \mathrm{ppm} ;{ }^{13} \mathrm{C} \mathrm{NMR} \mathrm{(125} \mathrm{MHz,} \mathrm{CDCl}_{3}\right)$ : $\delta 200.2(\mathrm{~d}, J=72.0 \mathrm{~Hz}), 142.2,133.9(\mathrm{~d}, J=49.3 \mathrm{~Hz}), 133.5(\mathrm{~d}, J=2.8 \mathrm{~Hz}), 132.4(\mathrm{~d}$, $J=8.3 \mathrm{~Hz}), 131.1,129.6,128.9(\mathrm{~d}, J=11.9 \mathrm{~Hz}), 128.7 \mathrm{ppm}$; ESI-MS: $\mathrm{m} / z 403.0$ $\left(\mathrm{M}^{+}+\mathrm{H}\right)$; HRMS calcd for $\mathrm{C}_{20} \mathrm{H}_{14} \mathrm{O}_{3} \mathrm{Cl}_{2} \mathrm{P}[\mathrm{M}+\mathrm{H}]:$ 403.0052, found 403.0053 .

\section{Synthesis and characterization of products}

\section{Typical procedure for the hydroacylation of unactivated alkenes.}

To a dried sealed tube $(25 \mathrm{~mL})$ equipped with a Teflon septum and magnetic stir bar were added successively $\mathrm{Co}\left(\mathrm{Sal}^{t-\mathrm{Bu}, t-\mathrm{Bu}}\right)(2,12.0 \mathrm{mg}, 0.02 \mathrm{mmol})$, Selectfluor $(14.2$ mg, $0.040 \mathrm{mmol}$ ), (4-chlorophenyl)(5,5-dimethyl-2-oxido-1,3,2-dioxaphosphinan-2yl)methanone (3a, $201.6 \mathrm{mg}, 0.70 \mathrm{mmol})$, The mixture was then evacuated and backfilled with argon (3 times). $i$-PrOH (6 mL), but-3-en-1-ylbenzene (1a, $30 \mu \mathrm{L}, 0.20$ mmol), TBHP (5.5 $\mathrm{M}$ in decane, $55 \mu \mathrm{L}, 0.30 \mathrm{mmol})$ and $\mathrm{PhSiH}_{3}(50 \mu \mathrm{L}, 0.40 \mathrm{mmol})$ were added successively. The reaction mixture was stirred at $\mathrm{rt}$ for $12 \mathrm{~h}$ and then concentrated under reduced pressure. The residue was purified by flash column chromatography on silica gel with EtOAc/petroleum ether $(1: 200)$ as the eluent to give product 4a as a colorless oil. Yield: $42.4 \mathrm{mg}(78 \%)$.

\section{Characterization of products}




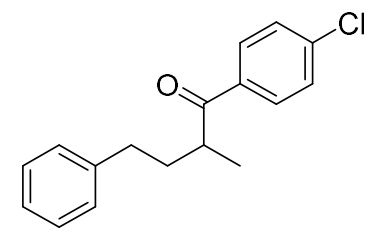

1-(4-Chlorophenyl)-2-methyl-4-phenylbutan-1-one (4a). Colorless oil; IR (neat): $v$ $\left(\mathrm{cm}^{-1}\right)$ 3064, 3032, 2968, 2930, 1681, 1589, 1453, 1400, 1222, 1092, 975, 842, 748, 700; ${ }^{1} \mathrm{H} \mathrm{NMR}\left(400 \mathrm{MHz}, \mathrm{CDCl}_{3}\right): \delta 7.77(\mathrm{~d}, J=8.4 \mathrm{~Hz}, 2 \mathrm{H}), 7.40(\mathrm{~d}, J=8.4 \mathrm{~Hz}, 2 \mathrm{H})$, $7.28(\mathrm{t}, J=7.6 \mathrm{~Hz}, 2 \mathrm{H}), 7.20(\mathrm{t}, J=7.6 \mathrm{~Hz}, 1 \mathrm{H}), 7.14$ (d, $J=6.8 \mathrm{~Hz}, 2 \mathrm{H}), 3.39$ (sextet, $J=6.8 \mathrm{~Hz}, 1 \mathrm{H}), 2.64(\mathrm{t}, J=7.6 \mathrm{~Hz}, 2 \mathrm{H}), 2.09-2.20(\mathrm{~m}, 1 \mathrm{H}), 1.69-1.79(\mathrm{~m}, 1 \mathrm{H}), 1.22$ $(\mathrm{d}, J=6.8 \mathrm{~Hz}, 3 \mathrm{H}) \mathrm{ppm} ;{ }^{13} \mathrm{C} \mathrm{NMR}\left(101 \mathrm{MHz}, \mathrm{CDCl}_{3}\right): \delta 202.8,141.5,139.3,134.7$, 129.7, 128.9, 128.5, 128.4, 126.0, 39.6, 35.1, 33.4, 17.1 ppm; ESI-MS: $m / z 295.0$ $\left(\mathrm{M}^{+}+\mathrm{Na}\right)$; HRMS calcd for $\mathrm{C}_{17} \mathrm{H}_{18} \mathrm{ClO}[\mathrm{M}+\mathrm{H}]: 273.1041$, found 273.1041 .

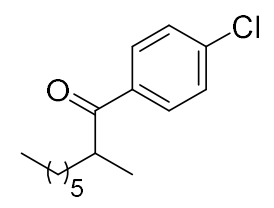

1-(4-Chlorophenyl)-2-methyloctan-1-one (4b). Colorless oil; IR (neat): v ( $\left.\mathrm{cm}^{-1}\right) 2957$, 2928, 2856, 1684, 1590,1570, 1460, 1400, 1376, 1219, 1093, 972, 841; ${ }^{1} \mathrm{H}$ NMR (400 $\left.\mathrm{MHz} \mathrm{CDCl}_{3}\right): \delta 7.89(\mathrm{~d}, J=8.8 \mathrm{~Hz}, 2 \mathrm{H}), 7.44(\mathrm{~d}, J=8.8 \mathrm{~Hz}, 2 \mathrm{H}), 3.40$ (sextet, $J=6.8$ $\mathrm{Hz}, 1 \mathrm{H}), 1.72-1.83(\mathrm{~m}, 1 \mathrm{H}), 1.37-1.48(\mathrm{~m}, 1 \mathrm{H}), 1.21-1.34(\mathrm{~m}, 8 \mathrm{H}), 1.18(\mathrm{~d}, J=6.8$ $\mathrm{Hz}, 3 \mathrm{H}), 0.86(\mathrm{t}, J=6.8 \mathrm{~Hz}, 3 \mathrm{H}) \mathrm{ppm} ;{ }^{13} \mathrm{C} \mathrm{NMR}\left(101 \mathrm{MHz}, \mathrm{CDCl}_{3}\right): \delta 203.3,139.2$, 135.0, 129.7, 128.9, 40.6, 33.7, 31.6, 29.4, 27.3, 22.6, 17.1, 14.0 ppm; ESI-MS: $m / z$ $274.3\left(\mathrm{M}^{+}+\mathrm{Na}\right)$; HRMS calcd for $\mathrm{C}_{15} \mathrm{H}_{21} \mathrm{ClO}[\mathrm{M}]: 252.1281$, found 252.1286.<smiles>CCC(C)C(=O)c1ccc(Cl)cc1</smiles>

1-(4-Chlorophenyl)-2-methyltetradecan-1-one (4c). Colorless oil; IR (neat): $v\left(\mathrm{~cm}^{-1}\right)$ 2925, 2853, 1685, 1590, 1464, 1400, 1376, 1219, 1093, 973, 841; ${ }^{1} \mathrm{H}$ NMR (400 MHz, $\left.\mathrm{CDCl}_{3}\right): \delta 7.89(\mathrm{~d}, J=8.8 \mathrm{~Hz}, 2 \mathrm{H}), 7.44(\mathrm{~d}, J=8.8 \mathrm{~Hz}, 2 \mathrm{H}), 3.40($ sextet, $J=6.8 \mathrm{~Hz}$, 
1H), $1.71-1.84(\mathrm{~m}, 1 \mathrm{H}), 1.36-1.48(\mathrm{~m}, 1 \mathrm{H}), 1.20-1.35$ (m, 20H), 1.18 (d, $J=6.8 \mathrm{~Hz}$, $3 \mathrm{H}), 0.88(\mathrm{t}, J=6.8 \mathrm{~Hz}, 3 \mathrm{H}) \mathrm{ppm} ;{ }^{13} \mathrm{C} \mathrm{NMR}\left(101 \mathrm{MHz}, \mathrm{CDCl}_{3}\right): \delta 203.3,139.2,135.0$, 129.7, 128.9, 40.6, 33.7, 31.9, 29.7, 29.6, 29.5, 29.3, 27.3, 22.7, 17.1, 14.1 ppm; EIMS: $m / z$ (rel intensity) $336\left(\mathrm{M}^{+}, 1\right), 170$ (31), 169 (11), 168 (89), 141 (36), 140 (9), 139 (100), 111 (14), 55 (6); HRMS calcd for $\mathrm{C}_{21} \mathrm{H}_{33} \mathrm{ClO}$ [M]: 336.2220, found 336.2218.<smiles>CC(NCl)C(=O)c1ccc(Cl)cc1</smiles>

11-Chloro-1-(4-chlorophenyl)-2-methylundecan-1-one (4d). Colorless oil; IR (neat): $v\left(\mathrm{~cm}^{-1}\right) 2929,2854,1685,1590,1461,1400,1219,1093,1014,973,842,747 ;{ }^{1} \mathrm{H}$ NMR (400 MHz, $\left.\mathrm{CDCl}_{3}\right): \delta 7.89(\mathrm{~d}, J=8.8 \mathrm{~Hz}, 2 \mathrm{H}), 7.44(\mathrm{~d}, J=8.8 \mathrm{~Hz}, 2 \mathrm{H}), 3.52$ (t, $J=6.8 \mathrm{~Hz}, 2 \mathrm{H}), 3.41$ (sextet, $J=6.8 \mathrm{~Hz}, 1 \mathrm{H}), 1.71-1.82(\mathrm{~m}, 3 \mathrm{H}), 1.35-1.48(\mathrm{~m}, 3 \mathrm{H})$, $1.22-1.34(\mathrm{~m}, 10 \mathrm{H}), 1.18(\mathrm{~d}, J=6.8 \mathrm{~Hz}, 3 \mathrm{H}) \mathrm{ppm} ;{ }^{13} \mathrm{C} \mathrm{NMR}\left(101 \mathrm{MHz}, \mathrm{CDCl}_{3}\right): \delta$ 203.3, 139.2, 135.0, 129.7, 128.9, 45.1, 40.6, 33.6, 32.6, 29.6, 29.3, 28.8, 27.3, 26.8, 17.2 ppm; EIMS: $m / z$ (rel intensity) $328\left(\mathrm{M}^{+}, 1\right), 170$ (23), 169 (8), 168 (67), 141 (36), 140 (9), 139 (100), 111 (15), 55 (7); HRMS calcd for $\mathrm{C}_{18} \mathrm{H}_{26} \mathrm{Cl}_{2} \mathrm{O}$ [M]: 328.1361, found 328.1367.<smiles>CC(NCBr)C(=O)c1ccc(Cl)cc1</smiles>

8-Bromo-1-(4-chlorophenyl)-2-methyloctan-1-one (4e). Colorless oil; IR (neat): v $\left(\mathrm{cm}^{-1}\right)$ 2976, 2933, 2856, 1683, 1589, 1570, 1487, 1460, 1400, 1375, 1224, 1092, 973 , 842; ${ }^{1} \mathrm{H}$ NMR (400 MHz, $\left.\mathrm{CDCl}_{3}\right): \delta 7.89(\mathrm{~d}, J=8.8 \mathrm{~Hz}, 2 \mathrm{H}), 7.45(\mathrm{~d}, J=8.8 \mathrm{~Hz}, 2 \mathrm{H})$, 3.35-3.46 (m, 3H), 1.74-1.86 (m, 3H), 1.36-1.49 (m, 3H), 1.27-1.35 (m, 4H), 1.19 (d, $J=6.8 \mathrm{~Hz}, 3 \mathrm{H}) \mathrm{ppm} ;{ }^{13} \mathrm{C} \mathrm{NMR}\left(101 \mathrm{MHz}, \mathrm{CDCl}_{3}\right): \delta 203.1,139.3,134.9129 .6,128.9$, 40.5, 33.9, 33.4, 32.6, 28.8, 27.9, 27.1, 17.2 ppm; EIMS: $m / z$ (rel intensity) 170 (18), 169 (7), 168 (52), 141 (36), 140 (10), 139 (100), 113 (6), 111 (17); HRMS calcd for $\mathrm{C}_{15} \mathrm{H}_{21} \mathrm{BrClO}[\mathrm{M}+\mathrm{H}]:$ 331.0459, found 331.0450. 
<smiles>CC(C)OCCCC(C)C(=O)c1ccc(Cl)cc1</smiles>

5-(4-Chlorophenyl)-4-methyl-5-oxopentyl 4-methylbenzenesulfonate

(4f).

Colorless oil; IR (neat): $v\left(\mathrm{~cm}^{-1}\right)$ 3066, 2968, 2930, 1682, 1589, 1570, 1358, 1175, 1094, 971, 920, 814, 748, 663; ${ }^{1} \mathrm{H}$ NMR (400 MHz, $\left.\mathrm{CDCl}_{3}\right): \delta 7.86(\mathrm{~d}, J=8.8 \mathrm{~Hz}, 2 \mathrm{H}), 7.76$ $(\mathrm{d}, J=8.4 \mathrm{~Hz}, 2 \mathrm{H}), 7.44(\mathrm{~d}, J=8.8 \mathrm{~Hz}, 2 \mathrm{H}), 7.33(\mathrm{~d}, J=8.4 \mathrm{~Hz}, 2 \mathrm{H}), 4.02$ (t, $J=6.0$ $\mathrm{Hz}, 2 \mathrm{H}), 3.40$ (sextet, $J=6.8 \mathrm{~Hz}, 1 \mathrm{H}), 2.44(\mathrm{~s}, 3 \mathrm{H}), 1.74-1.88(\mathrm{~m}, 1 \mathrm{H}), 1.60-1.71(\mathrm{~m}$, $2 \mathrm{H}), 1.44-1.55(\mathrm{~m}, 1 \mathrm{H}), 1.16(\mathrm{~d}, J=6.8 \mathrm{~Hz}, 3 \mathrm{H}) \mathrm{ppm} ;{ }^{13} \mathrm{C} \mathrm{NMR}\left(101 \mathrm{MHz}, \mathrm{CDCl}_{3}\right)$ : $\delta 202.3,144.8,139.5,134.5,133.0,129.8,129.7,129.0,127.8,70.2,39.8,29.1,26.5$, 21.6, 17.5 ppm; ESI-MS: $m / z$ 398.1 $\left(\mathrm{M}^{+}+\mathrm{NH}_{4}\right)$; HRMS calcd for $\mathrm{C}_{19} \mathrm{H}_{25} \mathrm{ClNO}_{4} \mathrm{~S}[\mathrm{M}+$ $\left.\mathrm{NH}_{4}\right]$ : 398.1187, found 398.1186.<smiles>CC(CCN)C(=O)c1ccc(Cl)cc1</smiles>

$\mathrm{N}$-(4-(4-Chlorophenyl)-3-methyl-4-oxobutyl)-4-methylbenzenesulfonamide (4g). Colorless oil; IR (neat): $v\left(\mathrm{~cm}^{-1}\right)$ 3280, 3076, 2972, 2932, 1681, 1589, 1570, 1488, 1325 , $1233,1159,1092,977,843,814,663,551 ;{ }^{1} \mathrm{H} \mathrm{NMR}\left(400 \mathrm{MHz}, \mathrm{CDCl}_{3}\right): \delta 7.86(\mathrm{~d}, J=$ $8.4 \mathrm{~Hz}, 2 \mathrm{H}), 7.68(\mathrm{~d}, J=8.4 \mathrm{~Hz}, 2 \mathrm{H}), 7.42(\mathrm{~d}, J=8.4 \mathrm{~Hz}, 2 \mathrm{H}), 7.25(\mathrm{~d}, J=8.4 \mathrm{~Hz}, 2 \mathrm{H})$, $4.72(\mathrm{t}, J=6.4 \mathrm{~Hz}, 1 \mathrm{H}), 3.56$ (sextet, $J=6.8 \mathrm{~Hz}, 1 \mathrm{H}), 2.90-3.04(\mathrm{~m}, 2 \mathrm{H}), 2.40(\mathrm{~s}, 3 \mathrm{H})$, 1.99-2.10 (m, 1H), 1.55-1.66 (m, 1H), $1.15(\mathrm{~d}, J=6.8 \mathrm{~Hz}, 3 \mathrm{H}) \mathrm{ppm} ;{ }^{13} \mathrm{C}$ NMR $(101$ $\left.\mathrm{MHz}, \mathrm{CDCl}_{3}\right): \delta 202.4,143.4,139.6,136.6,134.3,129.8,129.7,129.0,127.0,41.1$, 37.8, 32.4, 21.5, 17.8 ppm; ESI-MS: $m / z 366.1\left(\mathrm{M}^{+}+\mathrm{H}\right)$; HRMS calcd for $\mathrm{C}_{18} \mathrm{H}_{24} \mathrm{ClN}_{2} \mathrm{O}_{3} \mathrm{~S}$ [M+NH 4$]$ : 383.1191, found 383.1211.<smiles>CC(C)COCCCC(C)C(=O)c1ccc(Cl)cc1</smiles> 
Colorless oil; IR (neat): $v\left(\mathrm{~cm}^{-1}\right)$ 3070, 2959, 2930, 2857, 1685, 1589, 1570, 1472, 1427, 1237, 1111, 1092, 979, 913, 843, 737, 702; ${ }^{1} \mathrm{H}$ NMR (400 MHz, $\left.\mathrm{CDCl}_{3}\right): \delta 7.96$ (d, $J=$ $8.4 \mathrm{~Hz}, 2 \mathrm{H}), 7.63$ (d, $J=6.8 \mathrm{~Hz}, 2 \mathrm{H}), 7.60$ (d, $J=6.8 \mathrm{~Hz}, 2 \mathrm{H}), 7.30-7.46$ (m, 8H), $3.65-3.83(\mathrm{~m}, 3 \mathrm{H}), 2.05-2.16(\mathrm{~m}, 1 \mathrm{H}), 1.53-1.63(\mathrm{~m}, 1 \mathrm{H}), 1.16(\mathrm{~d}, J=7.2 \mathrm{~Hz}, 3 \mathrm{H})$, 1.04 (s, 9H) ppm; ${ }^{13} \mathrm{C}$ NMR (101 MHz, $\left.\mathrm{CDCl}_{3}\right): \delta 202.9,139.2,135.5,134.7,133.5$, $129.9,129.7,129.6,128.8,127.7,127.6,61.4,36.8,36.3,26.8,19.2,16.7$ ppm; ESIMS: $m / z 473.20\left(\mathrm{M}^{+}+\mathrm{Na}\right)$; HRMS calcd for $\mathrm{C}_{27} \mathrm{H}_{32} \mathrm{ClO}_{2} \mathrm{Si}[\mathrm{M}+\mathrm{H}]$ : 451.1855, found 451.1853 .

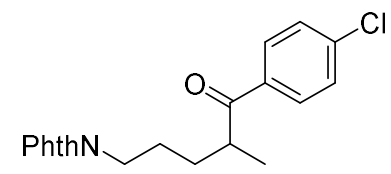

2-(5-(4-Chlorophenyl)-4-methyl-5-oxopentyl)isoindoline-1,3-dione (4i). Colorless oil; IR (neat): $v\left(\mathrm{~cm}^{-1}\right) 2935,1772,1712,1681,1589,1466,1397,1210,1092,973,884$, 720; ${ }^{1} \mathrm{H}$ NMR (400 MHz, $\left.\mathrm{CDCl}_{3}\right): \delta 7.88(\mathrm{~d}, J=8.0 \mathrm{~Hz}, 2 \mathrm{H}), 7.80-7.85(\mathrm{~m}, 2 \mathrm{H})$, $7.68-7.75(\mathrm{~m}, 2 \mathrm{H}), 7.42(\mathrm{~d}, J=8.0 \mathrm{~Hz}, 2 \mathrm{H}), 3.70(\mathrm{t}, J=6.8 \mathrm{~Hz}, 2 \mathrm{H}), 3.48$ (sextet, $J=$ $6.8 \mathrm{~Hz}, 1 \mathrm{H}), 1.80-1.91(\mathrm{~m}, 1 \mathrm{H}), 1.65-1.75(\mathrm{~m}, 2 \mathrm{H}), 1.44-1.55(\mathrm{~m}, 1 \mathrm{H}), 1.19$ (d, $J=$ $6.8 \mathrm{~Hz}, 3 \mathrm{H}) \mathrm{ppm} ;{ }^{13} \mathrm{C} \mathrm{NMR}\left(101 \mathrm{MHz}, \mathrm{CDCl}_{3}\right): \delta 202.6,168.3,139.3,134.7,133.9$, 132.0, 129.7, 128.9, 123.2, 40.0, 37.7, 30.3, 26.2, 17.4 ppm; ESI-MS: $m / z 356.2$ $\left(\mathrm{M}^{+}+\mathrm{H}\right)$; HRMS calcd for $\mathrm{C}_{20} \mathrm{H}_{19} \mathrm{ClNO}_{3}[\mathrm{M}+\mathrm{H}]: 356.1048$, found 356.1072.<smiles>CC(N=O)C(=O)c1ccc(Cl)cc1</smiles>

1-(4-Chlorophenyl)-2-methyl-7-nitroheptan-1-one (4j). Colorless oil; IR (neat): $v$ $\left(\mathrm{cm}^{-1}\right)$ 2934, 2860, 1682, 1590, 1552, 1487, 1380, 1220, 1092, 975, 843, 748; ${ }^{1} \mathrm{H}$ NMR $\left(400 \mathrm{MHz}, \mathrm{CDCl}_{3}\right): \delta 7.89(\mathrm{~d}, J=8.4 \mathrm{~Hz}, 2 \mathrm{H}), 7.45(\mathrm{~d}, J=8.4 \mathrm{~Hz}, 2 \mathrm{H}), 4.36(\mathrm{t}, J=6.8$ $\mathrm{Hz}, 2 \mathrm{H}), 3.41$ (sextet, $J=6.8 \mathrm{~Hz}, 1 \mathrm{H}), 1.94-2.04(\mathrm{~m}, 2 \mathrm{H}), 1.76-1.88(\mathrm{~m}, 1 \mathrm{H})$, $1.30-1.51(\mathrm{~m}, 5 \mathrm{H}), 1.19(\mathrm{~d}, J=6.8 \mathrm{~Hz}, 3 \mathrm{H}) \mathrm{ppm} ;{ }^{13} \mathrm{C} \mathrm{NMR}\left(101 \mathrm{MHz}, \mathrm{CDCl}_{3}\right): \delta 202.8$, 
139.4, 134.8, 129.6, 129.0, 75.5, 40.4, 33.0, 27.1, 26.5, 26.3, 17.4 ppm; ESI-MS: $m / z$ $282.0\left(\mathrm{M}^{+}-\mathrm{H}\right)$; HRMS calcd for $\mathrm{C}_{14} \mathrm{H}_{19} \mathrm{ClNO}_{3}[\mathrm{M}+\mathrm{H}]: 284.1048$, found 284.1047.<smiles>CC(C=O)C(=O)c1ccc(Cl)cc1</smiles>

11-(4-Chlorophenyl)-10-methyl-11-oxoundecanal (4k). Colorless oil. IR (neat): $v$ $\left(\mathrm{cm}^{-1}\right)$ 2928, 2854, 2713, 1724, 1683, 1589, 1570, 1487, 1459, 1400, 1374, 1220, 1092 , 1013, 973, 842; ${ }^{1} \mathrm{H}$ NMR (400 MHz, $\left.\mathrm{CDCl}_{3}\right): \delta 9.76(\mathrm{t}, J=2.0 \mathrm{~Hz}, 1 \mathrm{H}), 7.89$ (d, $J=$ $8.8 \mathrm{~Hz}, 2 \mathrm{H}), 7.44(\mathrm{~d}, J=8.8 \mathrm{~Hz}, 2 \mathrm{H}), 3.40$ (sextet, $J=6.8 \mathrm{~Hz}, 1 \mathrm{H}), 2.41(\mathrm{td}, J=7.6$, $2.0 \mathrm{~Hz}, 2 \mathrm{H}), 1.71-1.83(\mathrm{~m}, 1 \mathrm{H}), 1.55-1.66(\mathrm{~m}, 2 \mathrm{H}), 1.37-1.48(\mathrm{~m}, 1 \mathrm{H}), 1.22-1.34(\mathrm{~m}$, $10 \mathrm{H}), 1.18(\mathrm{~d}, J=6.8 \mathrm{~Hz}, 3 \mathrm{H}) \mathrm{ppm} ;{ }^{13} \mathrm{C} \mathrm{NMR}\left(101 \mathrm{MHz}, \mathrm{CDCl}_{3}\right): \delta 203.3,203.0,139.2$, 135.0, 129.7, 129.0, 43.9, 40.6, 33.6, 30.0, 29.6, 29.2, 29.1, 27.3, 22.0, 17.2 ppm; ESIMS: $m / z 307.2\left(\mathrm{M}^{+}-\mathrm{H}\right)$; HRMS calcd for $\mathrm{C}_{18} \mathrm{H}_{26} \mathrm{ClO}_{2}[\mathrm{M}+\mathrm{H}]$ : 309.1616, found 309.1626.<smiles>CCNC(=O)CC(C)C(=O)c1ccc(Cl)cc1</smiles>

11-(4-Chlorophenyl)- $N, N$-diethyl-10-methyl-11-oxoundecanamide (4l). Colorless oil; IR (neat): $v\left(\mathrm{~cm}^{-1}\right)$ 2971, 2930, 2854, 1683, 1641, 1589, 1460, 1429, 1400, 1363, 1222, 1138, 1092, 972, 842, 746; ${ }^{1} \mathrm{H}$ NMR (400 MHz, $\left.\mathrm{CDCl}_{3}\right): \delta 7.89$ (d, $J=8.4 \mathrm{~Hz}$, 2H), $7.44(\mathrm{~d}, J=8.4 \mathrm{~Hz}, 2 \mathrm{H}), 3.25-3.46(\mathrm{~m}, 5 \mathrm{H}), 2.27$ (t, $J=7.6 \mathrm{~Hz}, 2 \mathrm{H}), 1.70-1.80$ (m, 1H), 1.58-1.68 (m, 2H), 1.35-1.45 (m, 1H), 1.20-1.40 (m, 10H), $1.17(\mathrm{t}, J=7.2$ $\mathrm{Hz}, 6 \mathrm{H}), 1.10$ (d, $J=7.2 \mathrm{~Hz}, 3 \mathrm{H}) \mathrm{ppm} ;{ }^{13} \mathrm{C} \mathrm{NMR}\left(101 \mathrm{MHz}, \mathrm{CDCl}_{3}\right): \delta 203.3,172.2$, 139.2, 135.0, 129.6, 128.9, 41.9, 40.6, 40.0, 33.6, 33.1, 29.6, 29.4, 29.3, 29.2 2 27.3, 25.4, 17.1, 14.4, 13.1 ppm; ESI-MS: $m / z 380.3\left(\mathrm{M}^{+}+\mathrm{H}\right)$; HRMS calcd for $\mathrm{C}_{22} \mathrm{H}_{35} \mathrm{ClNO}_{2}$ $[\mathrm{M}+\mathrm{H}]: 380.2351$, found 380.2348 . 


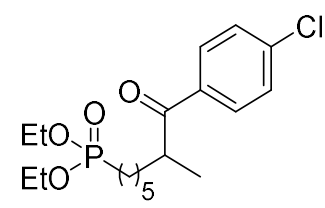

Diethyl (7-(4-chlorophenyl)-6-methyl-7-oxoheptyl)phosphonate (4m). Colorless oil; IR (neat): $v\left(\mathrm{~cm}^{-1}\right) 2979,2934,2861,1683,1589,1460,1400,1240,1165,1092,1030$, 962, 747; ${ }^{1} \mathrm{H}$ NMR (400 MHz, $\left.\mathrm{CDCl}_{3}\right): \delta 7.88(\mathrm{~d}, J=8.0 \mathrm{~Hz}, 2 \mathrm{H}), 7.43(\mathrm{~d}, J=8.0 \mathrm{~Hz}$, 2H), 3.99-4.16 (m, 4H), 3.39 (sextet, $J=6.8 \mathrm{~Hz}, 1 \mathrm{H}), 1.48-1.85(\mathrm{~m}, 6 \mathrm{H}), 1.35-1.48$ (m, 2H), 1.24-1.35 (m, 8H), $1.17(\mathrm{~d}, J=7.2 \mathrm{~Hz}, 3 \mathrm{H}) \mathrm{ppm} ;{ }^{13} \mathrm{C} \mathrm{NMR}\left(101 \mathrm{MHz}, \mathrm{CDCl}_{3}\right)$ : $\delta 203.1,139.3,134.9,129.6,128.9,61.4(\mathrm{~d}, J=7.3 \mathrm{~Hz}), 40.5,33.3,30.6(\mathrm{~d}, J=16.5$ $\mathrm{Hz}), 26.8,25.5$ (d, $J=141.3 \mathrm{~Hz}), 22.2(\mathrm{~d}, J=5.5 \mathrm{~Hz}), 17.3,16.4$ (d, $J=6.4 \mathrm{~Hz}) \mathrm{ppm}$; ESI-MS: $m / z$ 375.2 $\left(\mathrm{M}^{+}+\mathrm{H}\right)$; HRMS calcd for $\mathrm{C}_{18} \mathrm{H}_{29} \mathrm{ClO}_{4} \mathrm{P}[\mathrm{M}+\mathrm{H}]: 375.1487$, found 375.1470.

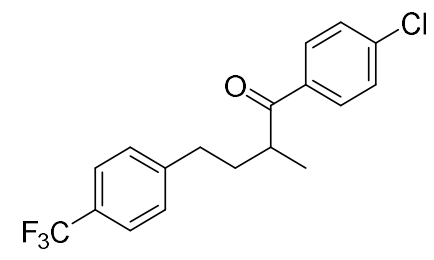

1-(4-Chlorophenyl)-2-methyl-4-(4-(trifluoromethyl)phenyl)butan-1-one

Colorless oil; IR (neat): $v\left(\mathrm{~cm}^{-1}\right)$ 2970, 2935, 1683, 1618, 1590, 1326, 1164, 1122, 1093, 976, 841, 748; ${ }^{1} \mathrm{H}$ NMR (400 MHz, $\left.\mathrm{CDCl}_{3}\right): \delta 7.81(\mathrm{~d}, J=8.4 \mathrm{~Hz}, 2 \mathrm{H}), 7.52$ (d, $J=7.6$ $\mathrm{Hz}, 2 \mathrm{H}), 7.43(\mathrm{~d}, J=8.4 \mathrm{~Hz}, 2 \mathrm{H}), 7.25$ (d, $J=7.6 \mathrm{~Hz}, 2 \mathrm{H}), 3.41$ (sextet, $J=6.8 \mathrm{~Hz}$, 1H), 2.60-2.76 (m, 2H), 2.12-2.25 (m, 1H), 1.71-1.83 (m, 1H), $1.24(\mathrm{~d}, J=6.8 \mathrm{~Hz}$, 3H) ppm; ${ }^{19} \mathrm{~F}$ NMR (376 MHz, $\left.\mathrm{CDCl}_{3}\right): \delta-62.3 \mathrm{ppm} ;{ }^{13} \mathrm{C} \mathrm{NMR}\left(101 \mathrm{MHz}, \mathrm{CDCl}_{3}\right): \delta$ 202.5, 145.8, 139.5, 134.7, 129.6, 129.0, 128.7, 128.4 (q, $J=32.8 \mathrm{~Hz}), 126.9$ (q, $J=$ 267.2 Hz), 125.3 (q, $J=3.6 \mathrm{~Hz}), 39.8,34.6,33.3,17.6$ ppm; ESI-MS: $m / z 339.2\left(\mathrm{M}^{+}-\mathrm{H}\right)$; HRMS calcd for $\mathrm{C}_{18} \mathrm{H}_{17} \mathrm{ClF}_{3} \mathrm{O}[\mathrm{M}+\mathrm{H}]: 341.0915$, found 341.0912 .

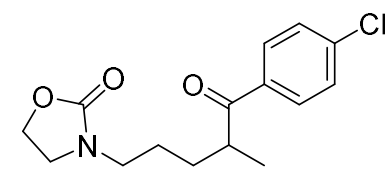


3-(5-(4-Chlorophenyl)-4-methyl-5-oxopentyl)oxazolidin-2-one (40). Colorless oil; IR (neat): $v\left(\mathrm{~cm}^{-1}\right)$ 2968, 2932, 2872, 1750, 1681, 1589, 1570, 1486, 1428, 1375, 1265 , 1219, 1092, 973, 843; ${ }^{1} \mathrm{H}$ NMR (400 MHz, $\left.\mathrm{CDCl}_{3}\right): \delta 7.91$ (d, $\left.J=8.8 \mathrm{~Hz}, 2 \mathrm{H}\right), 7.46$ (d, $J=8.8 \mathrm{~Hz}, 2 \mathrm{H}), 4.23-4.37(\mathrm{~m}, 2 \mathrm{H}), 3.45-3.60(\mathrm{~m}, 3 \mathrm{H}), 3.27(\mathrm{t}, J=6.8 \mathrm{~Hz}, 2 \mathrm{H})$, $1.79-1.90(\mathrm{~m}, 1 \mathrm{H}), 1.43-1.61(\mathrm{~m}, 3 \mathrm{H}), 1.20(\mathrm{~d}, J=6.8 \mathrm{~Hz}, 3 \mathrm{H}) \mathrm{ppm} ;{ }^{13} \mathrm{C}$ NMR $(101$ $\left.\mathrm{MHz}, \mathrm{CDCl}_{3}\right): \delta 202.7,158.5,139.5,134.6,129.7,129.0,61.6,44.3,43.9,39.8,29.9$, 24.8, 17.7 ppm; ESI-MS: m/z $296.1\left(\mathrm{M}^{+}+\mathrm{H}\right)$; HRMS calcd for $\mathrm{C}_{15} \mathrm{H}_{19} \mathrm{ClNO}_{3}[\mathrm{M}+\mathrm{H}]$ : 296.1048 , found 296.1048 .

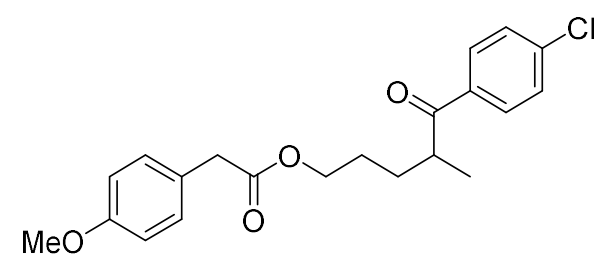

\section{5-(4-Chlorophenyl)-4-methyl-5-oxopentyl 2-(4-methoxyphenyl)acetate (4p).}

Colorless oil; IR (neat): $v\left(\mathrm{~cm}^{-1}\right)$ 2958, 1734, 1682, 1612, 1589, 1513, 1461, 1248, 1156 , 1092, 1035, 974; ${ }^{1} \mathrm{H}$ NMR (400 MHz, $\left.\mathrm{CDCl}_{3}\right): \delta 7.87$ (d, $\left.J=8.4 \mathrm{~Hz}, 2 \mathrm{H}\right), 7.44(\mathrm{~d}, J=$ $8.4 \mathrm{~Hz}, 2 \mathrm{H}), 7.17$ (d, $J=8.4 \mathrm{~Hz}, 2 \mathrm{H}), 6.84$ (d, $J=8.4 \mathrm{~Hz}, 2 \mathrm{H}), 4.07$ (t, $J=6.4 \mathrm{~Hz}, 2 \mathrm{H})$, $3.78(\mathrm{~s}, 3 \mathrm{H}), 3.53(\mathrm{~s}, 2 \mathrm{H}), 3.40$ (sextet, $J=6.8 \mathrm{~Hz}, 1 \mathrm{H}), 1.78-1.88(\mathrm{~m}, 1 \mathrm{H}), 1.58-1.67$ $(\mathrm{m}, 2 \mathrm{H}), 1.41-1.52(\mathrm{~m}, 1 \mathrm{H}), 1.18(\mathrm{~d}, J=6.8 \mathrm{~Hz}, 3 \mathrm{H}) \mathrm{ppm} ;{ }^{13} \mathrm{C} \mathrm{NMR}\left(101 \mathrm{MHz}, \mathrm{CDCl}_{3}\right)$ : $\delta 202.6,171.9,158.7,139.4,134.7,130.2,129.7,129.0,126.1,114.0,64.5,55.2,40.4$, 40.0, 29.5, 26.3, 17.4 ppm; ESI-MS: $m / z 397.2\left(\mathrm{M}^{+}+\mathrm{Na}\right)$; HRMS calcd for $\mathrm{C}_{21} \mathrm{H}_{24} \mathrm{ClO}_{4}$ $[\mathrm{M}+\mathrm{H}]:$ 375.1358, found 375.1357.

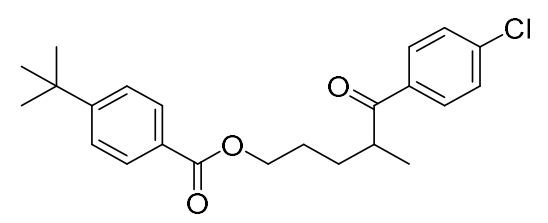

5-(4-Chlorophenyl)-4-methyl-5-oxopentyl 4-(tert-butyl)benzoate (4q). Colorless oil; IR (neat): $v\left(\mathrm{~cm}^{-1}\right)$ 2964, 1718, 1684, 1610, 1589, 1461, 1314, 1276, 1189, 1118, 1093, 1015, 974, 854, 756; ${ }^{1} \mathrm{H}$ NMR (400 MHz, $\left.\mathrm{CDCl}_{3}\right): \delta 7.91(\mathrm{~d}, J=8.4 \mathrm{~Hz}, 2 \mathrm{H}), 7.89$ (d, $J=8.4 \mathrm{~Hz}, 2 \mathrm{H}), 7.44(\mathrm{~d}, J=8.4 \mathrm{~Hz}, 2 \mathrm{H}), 7.40(\mathrm{~d}, J=8.4 \mathrm{~Hz}, 2 \mathrm{H}), 4.30(\mathrm{t}, J=6.4 \mathrm{~Hz}$, 2H), 3.49 (sextet, $J=6.8 \mathrm{~Hz}, 1 \mathrm{H}), 1.92-2.03(\mathrm{~m}, 1 \mathrm{H}), 1.74-1.83(\mathrm{~m}, 2 \mathrm{H}), 1.56-1.66$ 
(m, 1H), $1.34(\mathrm{~s}, 9 \mathrm{H}), 1.23(\mathrm{~d}, J=6.8 \mathrm{~Hz}, 3 \mathrm{H}) \mathrm{ppm} ;{ }^{13} \mathrm{C} \mathrm{NMR}\left(101 \mathrm{MHz}, \mathrm{CDCl}_{3}\right): \delta$ 202.6, 166.6, 156.6, 139.3, 134.6, 129.7, 129.4, 129.0, 127.4, 125.3, 64.3, 40.1, 35.0, 31.1, 29.8, 26.3, 17.2 ppm; ESI-MS: $m / z$ 409.2 $\left(\mathrm{M}^{+}+\mathrm{Na}\right)$; HRMS calcd for $\mathrm{C}_{23} \mathrm{H}_{28} \mathrm{ClO}_{3}$ $[\mathrm{M}+\mathrm{H}]:$ 387.1721, found 387.1746.

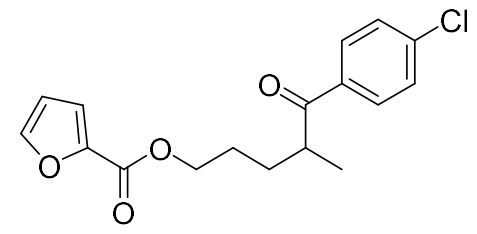

5-(4-Chlorophenyl)-4-methyl-5-oxopentyl furan-2-carboxylate (4r). Colorless oil; IR (neat): $v\left(\mathrm{~cm}^{-1}\right) 1726,1682,1589,1568,1475,1400,1296,1179,1120,1013,974$, 842; $\left.{ }^{1} \mathrm{H} \mathrm{NMR} \mathrm{(400} \mathrm{MHz,} \mathrm{CDCl}_{3}\right): \delta 7.87(\mathrm{~d}, J=8.4 \mathrm{~Hz}, 2 \mathrm{H}), 7.54(\mathrm{~d}, J=0.8 \mathrm{~Hz}, 1 \mathrm{H})$, $7.40(\mathrm{~d}, J=8.4 \mathrm{~Hz}, 2 \mathrm{H}), 7.11(\mathrm{~d}, J=3.6 \mathrm{~Hz}, 1 \mathrm{H}), 6.48(\mathrm{dd}, J=3.6,2.0 \mathrm{~Hz}, 1 \mathrm{H}), 4.27$ (t, $J=6.4 \mathrm{~Hz}, 2 \mathrm{H}), 3.47$ (sextet, $J=6.8 \mathrm{~Hz}, 1 \mathrm{H}), 1.89-2.00(\mathrm{~m}, 1 \mathrm{H}), 1.73-1.82$ (m, 2H), $1.54-1.64(\mathrm{~m}, 1 \mathrm{H}), 1.22(\mathrm{~d}, J=6.8 \mathrm{~Hz}, 3 \mathrm{H}) \mathrm{ppm} ;{ }^{13} \mathrm{C} \mathrm{NMR}\left(101 \mathrm{MHz}, \mathrm{CDCl}_{3}\right): \delta$ 202.6, 158.7, 146.3, 144.6, 139.4, 134.6, 129.7, 129.0, 117.9, 111.8, 64.6, 40.1, 29.6, 26.4, 17.4 ppm; EIMS: m/z (rel intensity) 208 (18), 165 (10), 141 (35), 140 (9), 139 (100), 113 (7), 111 (15), 95 (19); HRMS calcd for $\mathrm{C}_{17} \mathrm{H}_{18} \mathrm{ClO}_{4}[\mathrm{M}+\mathrm{H}]: 321.0888$, found 321.0890 .

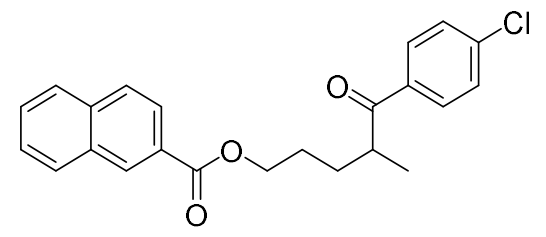

5-(4-Chlorophenyl)-4-methyl-5-oxopentyl 2-naphthoate (4s). Colorless oil; IR (neat): $v\left(\mathrm{~cm}^{-1}\right) 3060,2963,1715,1683,1631,1589,1463,1285,1228,1196,1093$, 973, 779; ${ }^{1} \mathrm{H}$ NMR (400 MHz, $\left.\mathrm{CDCl}_{3}\right): \delta 8.56$ (s, $\left.1 \mathrm{H}\right), 8.01(\mathrm{dd}, J=8.4,1.6 \mathrm{~Hz}, 1 \mathrm{H})$, 7.84-7.95 (m, 5H), 7.60 (td, $J=6.8,1.2 \mathrm{~Hz}, 1 \mathrm{H}), 7.55$ (td, $J=6.8,1.2 \mathrm{~Hz}, 1 \mathrm{H}), 7.40$ $(\mathrm{d}, J=8.4 \mathrm{~Hz}, 2 \mathrm{H}), 4.38(\mathrm{t}, J=6.4 \mathrm{~Hz}, 2 \mathrm{H}), 3.52$ (sextet, $J=6.8 \mathrm{~Hz}, 1 \mathrm{H}), 1.97-2.08$ (m, 1H), 1.80-1.89 (m, 2H), 1.60-1.71 (m, 1H), $1.25(\mathrm{~d}, J=6.8 \mathrm{~Hz}, 3 \mathrm{H}) \mathrm{ppm} ;{ }^{13} \mathrm{C}$ NMR (101 MHz, $\left.\mathrm{CDCl}_{3}\right): \delta 202.6,166.7,139.4,135.5,134.6,132.4,131.0,129.7$, 
129.3, 129.0, 128.2, 128.1, 127.7, 127.4, 126.6, 125.1, 64.8, 40.1, 29.8, 26.4, 17.4 ppm; EIMS: $m / z$ (rel intensity) $380\left(\mathrm{M}^{+}, 2\right), 225$ (14), 208 (8), 172 (19), 155 (34), 139 (100), 127 (37), 111 (13); HRMS calcd for $\mathrm{C}_{23} \mathrm{H}_{22} \mathrm{ClO}_{3}[\mathrm{M}+\mathrm{H}]$ : 381.1252, found 381.1258.

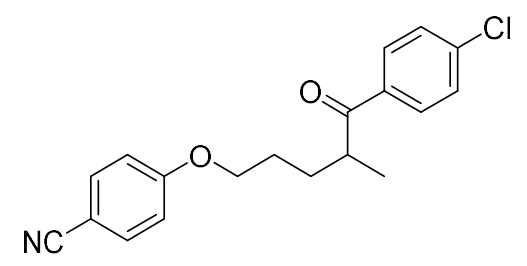

4-((5-(4-Chlorophenyl)-4-methyl-5-oxopentyl)oxy)benzonitrile (4t). Colorless oil; IR (neat): $v\left(\mathrm{~cm}^{-1}\right)$ 2935, 2224, 1681, 1606, 1589, 1509, 1400, 1302, 1259, 1194, 1092 , 974, 835; ${ }^{1} \mathrm{H}$ NMR (400 MHz, $\left.\mathrm{CDCl}_{3}\right): \delta 7.90(\mathrm{~d}, J=8.8 \mathrm{~Hz}, 2 \mathrm{H}), 7.56(\mathrm{~d}, J=8.8 \mathrm{~Hz}$, 2H), 7.45 (d, $J=8.8 \mathrm{~Hz}, 2 \mathrm{H}), 6.88$ (d, $J=8.8 \mathrm{~Hz}, 2 \mathrm{H}), 3.99$ (t, $J=6.4 \mathrm{~Hz}, 2 \mathrm{H}), 3.50$ (sextet, $J=6.8 \mathrm{~Hz}, 1 \mathrm{H}), 1.95-2.05(\mathrm{~m}, 1 \mathrm{H}), 1.75-1.88(\mathrm{~m}, 2 \mathrm{H}), 1.60-1.70(\mathrm{~m}, 1 \mathrm{H})$, $1.24(\mathrm{~d}, J=6.8 \mathrm{~Hz}, 3 \mathrm{H}) \mathrm{ppm} ;{ }^{13} \mathrm{C} \mathrm{NMR}\left(101 \mathrm{MHz}, \mathrm{CDCl}_{3}\right): \delta 202.5,162.1,139.5$, 134.6, 133.9, 129.7, 129.0, 119.2, 115.1, 103.8, 68.1 , 40.2 29.7, 26.7, 17.7 ppm; EIMS: $m / z$ (rel intensity) $327\left(\mathrm{M}^{+}, 1\right), 211$ (25), 209 (70), 141 (36), 140 (9), 139 (100), 113 (8), 111 (23), 102 (7), 75 (7); HRMS calcd for $\mathrm{C}_{19} \mathrm{H}_{19} \mathrm{ClNO}_{2}[\mathrm{M}+\mathrm{H}]$ : 328.1099, found 328.1104 .

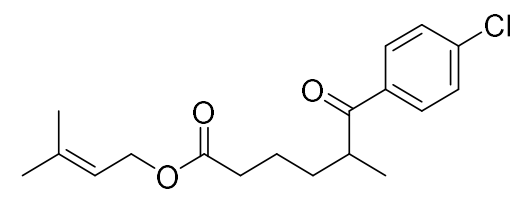

3-Methylbut-2-en-1-yl 6-(4-chlorophenyl)-5-methyl-6-oxohexanoate (4u). Colorless oil; IR (neat): $v\left(\mathrm{~cm}^{-1}\right)$ 2972, 2933, 1732, 1683, 1589, 1456, 1377, 1219, 1163 , 1092, 972, 841; ${ }^{1} \mathrm{H}$ NMR (400 MHz, $\mathrm{CDCl}_{3}$ ): $\delta 7.89$ (d, $\left.J=8.4 \mathrm{~Hz}, 2 \mathrm{H}\right), 7.44$ (d, $J=$ $8.4 \mathrm{~Hz}, 2 \mathrm{H}), 5.31$ (t, $J=7.2 \mathrm{~Hz}, 1 \mathrm{H}), 4.54$ (d, $J=7.2 \mathrm{~Hz}, 2 \mathrm{H}), 3.42$ (sextet, $J=6.8 \mathrm{~Hz}$, 1H), $2.30(\mathrm{t}, J=7.2 \mathrm{~Hz}, 2 \mathrm{H}), 1.77-1.87(\mathrm{~m}, 1 \mathrm{H}), 1.74(\mathrm{~s}, 3 \mathrm{H}), 1.69$ (s, 3H), 1.59-1.68 (m, 2H), 1.40-1.51 (m, 1H), $1.19(\mathrm{~d}, J=6.4 \mathrm{~Hz}, 3 \mathrm{H}) \mathrm{ppm} ;{ }^{13} \mathrm{C} \mathrm{NMR}\left(101 \mathrm{MHz}, \mathrm{CDCl}_{3}\right)$ : $\delta 202.7,173.3,139.4,139.1,134.7,129.7,129.0,118.5,61.3,40.4,34.2,32.8,25.7$, 22.8, 18.0, 17.2 ppm; ESI-MS: $m / z 345.15\left(\mathrm{M}^{+}+\mathrm{Na}\right)$; HRMS calcd for $\mathrm{C}_{18} \mathrm{H}_{24} \mathrm{ClO}_{3}$ $[\mathrm{M}+\mathrm{H}]:$ 323.1408, found 323.1403. 


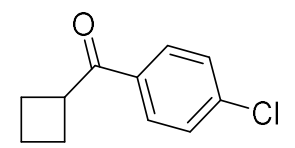

(4-Chlorophenyl)(cyclobutyl)methanone (4v). Colorless oil; IR (neat): $v\left(\mathrm{~cm}^{-1}\right)$ 2991, 2942, 1680, 1589, 1486, 1402, 1342, 1220, 1091, 1013, 967, 840; ${ }^{1} \mathrm{H}$ NMR (400 MHz, $\left.\mathrm{CDCl}_{3}\right): \delta 7.83(\mathrm{~d}, J=8.8 \mathrm{~Hz}, 2 \mathrm{H}), 7.41(\mathrm{~d}, J=8.8 \mathrm{~Hz}, 2 \mathrm{H}), 3.95$ (quintet, $J=8.4 \mathrm{~Hz}$, $1 \mathrm{H}), 2.34-2.46(\mathrm{~m}, 2 \mathrm{H}), 2.23-2.34(\mathrm{~m}, 2 \mathrm{H}), 2.02-2.15(\mathrm{~m}, 1 \mathrm{H}), 1.85-1.97(\mathrm{~m}, 1 \mathrm{H})$ ppm; ${ }^{13} \mathrm{C} \mathrm{NMR}\left(101 \mathrm{MHz}, \mathrm{CDCl}_{3}\right): \delta 199.8,139.2,133.9,129.7,128.9,42.1,25.0,18.1$ ppm; ESI-MS: $m / z$ 195.1 $\left(\mathrm{M}^{+}+\mathrm{H}\right)$; HRMS calcd for $\mathrm{C}_{11} \mathrm{H}_{12} \mathrm{ClO}[\mathrm{M}+\mathrm{H}]$ : 195.0571, found 195.0572 .

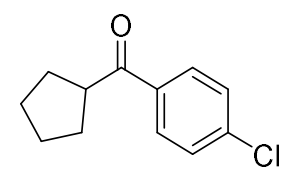

(4-Chlorophenyl)(cyclopentyl)methanone (4w). Colorless oil; IR (neat): $v\left(\mathrm{~cm}^{-1}\right)$ 2956, 2869, 1682, 1589, 1487, 1401, 1217, 1092, 1000, 840; ${ }^{1} \mathrm{H}$ NMR (400 MHz, $\mathrm{CDCl}_{3}$ ): $\delta 7.91(\mathrm{~d}, J=8.8 \mathrm{~Hz}, 2 \mathrm{H}), 7.43(\mathrm{~d}, J=8.0 \mathrm{~Hz}, 2 \mathrm{H}), 3.66$ (quintet, $J=8.0 \mathrm{~Hz}$, 1H), 1.83-1.96 (m, 4H), 1.59-1.78 (m, 4H) ppm; $\left.{ }^{13} \mathrm{C} \mathrm{NMR} \mathrm{(101} \mathrm{MHz,} \mathrm{CDCl}\right): \delta 201.5$, 139.1, 135.2, 129.9, 128.8, 46.3, 29.9, 26.3 ppm; ESI-MS: $m / z$ 209.1 $\left(\mathrm{M}^{+}+\mathrm{H}\right)$; HRMS calcd for $\mathrm{C}_{12} \mathrm{H}_{14} \mathrm{ClO}[\mathrm{M}+\mathrm{H}]: 209.0728$, found 209.0728.<smiles>O=C(c1ccc(Cl)cc1)C1CCCCC1</smiles>

(4-Chlorophenyl)(cyclohexyl)methanone (4x). Colorless oil; IR (neat): v ( $\left.\mathrm{cm}^{-1}\right)$ 2933, 2855, 1683, 1589, 1450, 1400, 1250, 1206, 1093, 975, 739; ${ }^{1} \mathrm{H}$ NMR (400 MHz, $\left.\mathrm{CDCl}_{3}\right): \delta 7.88(\mathrm{~d}, J=8.8 \mathrm{~Hz}, 2 \mathrm{H}), 7.43(\mathrm{~d}, J=8.4 \mathrm{~Hz}, 2 \mathrm{H}), 3.20(\mathrm{tt}, J=11.6,3.2 \mathrm{~Hz}$, 1H), 1.80-1.91 (m, 4H), 1.69-1.78 (m, 1H), 1.27-1.54 (m, 5H) ppm; ${ }^{13} \mathrm{C}$ NMR (101 $\left.\mathrm{MHz}, \mathrm{CDCl}_{3}\right): \delta$ 202.6, 139.1, 134.6, 129.7, 128.9, 45.6, 29.3, 25.9, 25.8 ppm; ESI-MS: $m / z 223.1\left(\mathrm{M}^{+}+\mathrm{H}\right)$; HRMS calcd for $\mathrm{C}_{13} \mathrm{H}_{16} \mathrm{ClO}[\mathrm{M}+\mathrm{H}]: 223.0884$, found 223.0883 
<smiles>CC(=O)C1CCC(C(=O)c2ccc(Cl)cc2)C1</smiles>

Methyl 3-(4-chlorobenzoyl)cyclopentane-1-carboxylate (4y). This compound was isolated as two separable isomers in 50:50 ratio determined by ${ }^{1} \mathrm{H}$ NMR (400 MHz).

One isomer: Colorless oil; IR (neat): $v\left(\mathrm{~cm}^{-1}\right)$ 2951, 1733, 1683, 1589, 1436, 1400, 1360, 1215, 1091, 844; ${ }^{1} \mathrm{H}$ NMR (400 MHz, $\left.\mathrm{CDCl}_{3}\right): \delta 7.90$ (d, $\left.J=8.4 \mathrm{~Hz}, 2 \mathrm{H}\right), 7.43$ (d, $J=8.4 \mathrm{~Hz}, 2 \mathrm{H}$ ), 3.85 (quintet, $J=7.2 \mathrm{~Hz}, 1 \mathrm{H}$ ), 3.69 (s, 3H), 2.96 (quintet, $J=7.2$ $\mathrm{Hz}, 1 \mathrm{H}), 2.15-2.27(\mathrm{~m}, 2 \mathrm{H}), 2.00-2.14(\mathrm{~m}, 2 \mathrm{H}), 1.84-1.98(\mathrm{~m}, 2 \mathrm{H}) \mathrm{ppm} ;{ }^{13} \mathrm{C} \mathrm{NMR}$ $\left(101 \mathrm{MHz}, \mathrm{CDCl}_{3}\right): \delta 200.5,176.4,139.4,134.6,129.9,128.9,51.8,45.7,43.4,32.6$, 30.1, 29.7 ppm; ESI-MS: m/z $267.1\left(\mathrm{M}^{+}+\mathrm{H}\right)$; HRMS calcd for $\mathrm{C}_{14} \mathrm{H}_{16} \mathrm{ClO}_{3}[\mathrm{M}+\mathrm{H}]$ : 267.0782, found 267.0781.

The other isomer: Colorless oil; IR (neat): $v\left(\mathrm{~cm}^{-1}\right)$ 2951, 1734, 1683, 1589, 1436, 1401, 1218, 1168, 1091, 843; ${ }^{1} \mathrm{H}$ NMR (400 MHz, $\left.\mathrm{CDCl}_{3}\right): \delta 7.88$ (d, $\left.J=8.4 \mathrm{~Hz}, 2 \mathrm{H}\right)$, $7.43(\mathrm{~d}, J=8.4 \mathrm{~Hz}, 2 \mathrm{H}), 3.65-3.76(\mathrm{~m}, 4 \mathrm{H}), 2.89$ (quintet, $J=8.0 \mathrm{~Hz}, 1 \mathrm{H}$ ), 2.17-2.31 (m, 2H), 1.92-2.11 (m, 4H) ppm; ${ }^{13} \mathrm{C} \mathrm{NMR}\left(101 \mathrm{MHz}, \mathrm{CDCl}_{3}\right): \delta$ 199.8, 175.4, 139.3, 134.8, 129.8, 128.9, 51.8, 46.2, 44.3, 33.0, 29.3, 29.2 ppm; ESI-MS: $m / z 267.1\left(\mathrm{M}^{+}+\mathrm{H}\right)$; HRMS calcd for $\mathrm{C}_{14} \mathrm{H}_{16} \mathrm{ClO}_{3}[\mathrm{M}+\mathrm{H}]:$ 267.0782, found 267.0782 .<smiles>CC(CCCOC(=O)C(Cc1ccccc1)NC(=O)CNCOC(C)(C)C)C(=O)c1ccc(Cl)cc1</smiles>

\section{5-(4-Chlorophenyl)-4-methyl-5-oxopentyl}

phenylalaninate (4za). This compound was isolated as a mixture of two stereoisomers in 50:50 ratio determined by ${ }^{1} \mathrm{H}$ NMR $(400 \mathrm{MHz})$. Colorless oil; IR (neat): $v\left(\mathrm{~cm}^{-1}\right)$ $3345,3064,2975,2933,1741,1716,1682,1589,1515,1455,1367,1173,1093,974$, 734; ${ }^{1} \mathrm{H}$ NMR (400 MHz, $\left.\mathrm{CDCl}_{3}\right): \delta 7.87 / 7.86(2 \mathrm{~d}, J=8.8 \mathrm{~Hz}, 2 \mathrm{H}), 7.43 / 7.42(2 \mathrm{~d}, J=$ $8.8 \mathrm{~Hz}, 2 \mathrm{H}), 7.14-7.27(\mathrm{~m}, 3 \mathrm{H}), 7.08$ (t, $J=7.2 \mathrm{~Hz}, 2 \mathrm{H}), 6.68(\mathrm{br}, 1 \mathrm{H}), 5.29$ (br, 1H), 4.77-4.86 (m, 1H), 3.90-4.15 (m, 2H), $3.81(\mathrm{dd}, J=16.8,4.4 \mathrm{~Hz}, 1 \mathrm{H}), 3.72(\mathrm{dd}, J=$ 
16.8, 5.6 Hz, 1H), 3.33-3.45 (m, 1H), 2.99-3.10 (m, 2H), 1.70-1.84 (m, 1H), 1.50-1.60 (m, 2H), 1.34-1.46 (m, 10H), $1.16(\mathrm{~d}, J=6.8 \mathrm{~Hz}, 3 \mathrm{H}) \mathrm{ppm} ;{ }^{13} \mathrm{C} \mathrm{NMR}(101$ $\left.\mathrm{MHz}, \mathrm{CDCl}_{3}\right): \delta 202.5 / 202.4,171.3 / 171.2,169.2 / 169.1,156.0,139.5,135.7 / 135.6$, 134.6/134.5, 129.7/129.6, 129.2/129.1, 129.0, 128.6/128.5, 127.1/127.0, 80.1, 65.2, 53.1, 44.1, 40.1/40.0, 38.0/37.9, 29.4/29.3, 28.2, 26.2/26.1, 17.7/17.6 ppm; ESI-MS: $m / z 531.2\left(\mathrm{M}^{+}+\mathrm{H}\right)$; HRMS calcd for $\mathrm{C}_{28} \mathrm{H}_{36} \mathrm{ClN}_{2} \mathrm{O}_{6}[\mathrm{M}+\mathrm{H}]:$ 531.2256, found 531.2253.

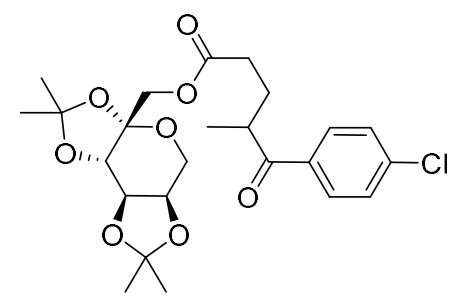

((3aS,5a $R, 8 \mathrm{a} R, 8 \mathrm{~b} S)-2,2,7,7-$ tetramethyltetrahydro-3aH-bis([1,3]dioxolo) $[4,5$ $\left.b: 4^{\prime}, 5^{\prime}-d\right]$ pyran-3a-yl)methyl 5-(4-chlorophenyl)-4-methyl-5 oxopentanoate (4zb). This compound was isolated as a mixture of two stereoisomers in 50:50 ratio determined by ${ }^{1} \mathrm{H}$ NMR (400 MHz). Colorless oil; IR (neat): $v\left(\mathrm{~cm}^{-1}\right)$ 2989, 2937, 1743 , $1683,1590,1456,1382,1252,1211,1072,980,888 ;{ }^{1} \mathrm{H}$ NMR (400 MHz, $\left.\mathrm{CDCl}_{3}\right): \delta$ $7.92(\mathrm{~d}, J=8.0 \mathrm{~Hz}, 2 \mathrm{H}), 7.45(\mathrm{~d}, J=8.0 \mathrm{~Hz}, 2 \mathrm{H}), 4.56-4.62(\mathrm{~m}, 1 \mathrm{H}), 4.42 / 4.41(2 \mathrm{~d}, J$ $=11.6 \mathrm{~Hz}, 1 \mathrm{H}), 4.26(\mathrm{~d}, J=8.4 \mathrm{~Hz}, 1 \mathrm{H}), 4.22(\mathrm{~d}, J=7.2 \mathrm{~Hz}, 1 \mathrm{H}), 4.03 / 4.01(2 \mathrm{~d}, J=$ $11.6 \mathrm{~Hz}, 1 \mathrm{H}), 3.90(\mathrm{~d}, J=12.8 \mathrm{~Hz}, 1 \mathrm{H}), 3.76(\mathrm{~d}, J=12.8 \mathrm{~Hz}, 1 \mathrm{H}), 3.56(\operatorname{sextet}, J=6.8$ $\mathrm{Hz}, 1 \mathrm{H}), 2.41-2.52(\mathrm{~m}, 1 \mathrm{H}), 2.31-2.41(\mathrm{~m}, 1 \mathrm{H}), 2.10-2.22(\mathrm{~m}, 1 \mathrm{H}), 1.73-1.84(\mathrm{~m}, 1 \mathrm{H})$, 1.54/1.51 (2s, 3H), $1.48(\mathrm{~s}, 3 \mathrm{H}), 1.37 / 1.35(2 \mathrm{~s}, 3 \mathrm{H}), 1.33 / 1.31(2 \mathrm{~s}, 3 \mathrm{H}), 1.20(\mathrm{~d}, J=6.8$ $\mathrm{Hz}, 3 \mathrm{H}) \mathrm{ppm} ;{ }^{13} \mathrm{C} \mathrm{NMR}\left(101 \mathrm{MHz}, \mathrm{CDCl}_{3}\right): \delta 202.3,172.5 / 172.4,139.5,134.6,129.8$, 129.0, 109.1, 108.7/108.6, 101.4, 70.7, 70.5/70.4, 70.0, 65.3/65.2, 61.3/61.2, 39.4/39.3, 31.3, 28.1/28.0, 26.5/26.4, 25.9, 25.2/25.1, 24.1/24.0, 17.3/17.2 ppm; ESI-MS: $\mathrm{m} / \mathrm{z}$ $505.2\left(\mathrm{M}^{+}+\mathrm{Na}\right)$; HRMS calcd for $\mathrm{C}_{24} \mathrm{H}_{31} \mathrm{ClNaO}_{8}[\mathrm{M}+\mathrm{H}]$ : 505.1600, found 505.1597.

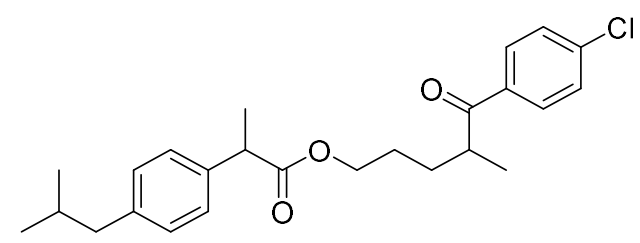




\section{5-(4-Chlorophenyl)-4-methyl-5-oxopentyl 2-(4-isobutylphenyl)propanoate (4zc).}

This compound was isolated as a 50:50 mixture of two stereoisomers determined by ${ }^{1} \mathrm{H}$ NMR (400 MHz). Colorless oil; IR (neat): $v\left(\mathrm{~cm}^{-1}\right)$ 2955, 1733, 1685, 1589, 1460, 1201 , 1166, 1093, 974, 843; ${ }^{1} \mathrm{H}$ NMR (400 MHz, $\mathrm{CDCl}_{3}$ ): $\delta 7.85$ (d, J=8.8 Hz, 2H), 7.43 (d, $J=8.8 \mathrm{~Hz}, 2 \mathrm{H}), 7.17(\mathrm{~d}, J=8.0 \mathrm{~Hz}, 2 \mathrm{H}), 7.06(\mathrm{~d}, J=8.0 \mathrm{~Hz}, 2 \mathrm{H}), 3.99-4.10(\mathrm{~m}, 2 \mathrm{H})$, $3.65(\mathrm{qd}, J=7.2,2.4 \mathrm{~Hz}, 1 \mathrm{H}), 3.30-3.41(\mathrm{~m}, 1 \mathrm{H}), 2.42(\mathrm{~d}, J=7.2 \mathrm{~Hz}, 2 \mathrm{H}), 1.71-1.88$ (m, 2H), 1.54-1.63 (m, 2H), 1.47/1.46 (2d, $J=7.2 \mathrm{~Hz}, 3 \mathrm{H}), 1.35-1.44(\mathrm{~m}, 1 \mathrm{H})$, $1.14 / 1.13(2 \mathrm{~d}, J=7.2 \mathrm{~Hz}, 3 \mathrm{H}), 0.88$ (d, $J=6.8 \mathrm{~Hz}, 6 \mathrm{H}) \mathrm{ppm} ;{ }^{13} \mathrm{C}$ NMR (101 MHz, $\left.\mathrm{CDCl}_{3}\right): \delta 202.5,174.7 / 174.6,140.5,139.4,137.7,134.7,129.6,129.3,128.9,127.1$, 64.3/64.2, 45.2/45.1, 45.0, 40.0/39.9, 30.1, 29.5, 26.2/26.1, 22.3, 18.4/18.3, 17.3/17.2 ppm; ESI-MS: $m / z$ 437.2 ( $\left.\mathrm{M}^{+}+\mathrm{Na}\right)$; HRMS calcd for $\mathrm{C}_{25} \mathrm{H}_{31} \mathrm{ClNaO}_{3}[\mathrm{M}+\mathrm{H}]$ : 437.1854, found 437.1849 .

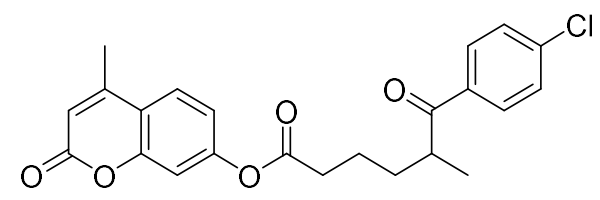

\section{4-Methyl-2-oxo-2H-chromen-7-yl 6-(4-chlorophenyl)-5-methyl-6-oxohexanoate} (4zd). Colorless oil; IR (neat): $v\left(\mathrm{~cm}^{-1}\right)$ 3080, 2930, 1761, 1733, 1682, 1614, 1589, 1387, 1263, 1131, 979, 855; ${ }^{1} \mathrm{H}$ NMR (400 MHz, $\left.\mathrm{CDCl}_{3}\right): \delta 7.90$ (d, J=8.4 Hz, 2H), 7.58 (d, $J=8.4 \mathrm{~Hz}, 1 \mathrm{H}), 7.44(\mathrm{~d}, J=8.4 \mathrm{~Hz}, 2 \mathrm{H}), 7.08(\mathrm{~d}, J=2.4 \mathrm{~Hz}, 1 \mathrm{H}), 7.02(\mathrm{dd}, J=8.8$, $2.4 \mathrm{~Hz}, 1 \mathrm{H}), 6.26$ (s, 1H), 3.47 (sextet, $J=6.8 \mathrm{~Hz}, 1 \mathrm{H}), 2.60$ (t, $J=7.6 \mathrm{~Hz}, 2 \mathrm{H}), 2.43$ (s, 3H), 1.88-1.99 (m, 1H), 1.51-1.63 (m, 2H), 1.71-1.88 (m, 1H), $1.23(\mathrm{~d}, J=6.8 \mathrm{~Hz}$, 3H) ppm; ${ }^{13} \mathrm{C}$ NMR $\left(101 \mathrm{MHz}, \mathrm{CDCl}_{3}\right): \delta 202.5,171.1,160.5,154.1,153.0,151.9$, $139.5,134.6,129.7,129.0,125.3,118.0,117.8,114.5,110.4,40.5,34.2,32.6,22.5$, 18.7, 17.5 ppm; ESI-MS: $m / z$ 413.1 $\left(\mathrm{M}^{+}+\mathrm{H}\right)$; HRMS calcd for $\mathrm{C}_{23} \mathrm{H}_{22} \mathrm{ClO}_{5}[\mathrm{M}+\mathrm{H}]$ : 413.1150, found 413.1146.

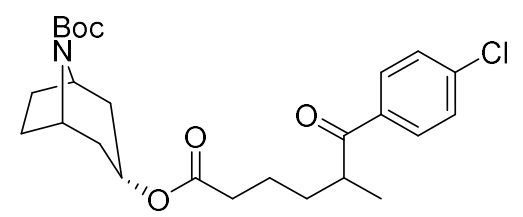


tert-Butyl (1R,3S,5S)-3-((6-(4-chlorophenyl)-5-methyl-6-oxohexanoyl)oxy)-8azabicyclo[3.2.1]octane-8-carboxylate (4ze). This compound was isolated as a 50:50 mixture of two stereoisomers determined by ${ }^{13} \mathrm{C}$ NMR $(101 \mathrm{MHz})$. Colorless oil; IR (neat): $v\left(\mathrm{~cm}^{-1}\right)$ 2973, 1730, 1694, 1589, 1400, 1366, 1162, 1094, 1032, 972; ${ }^{1} \mathrm{H}$ NMR $\left(400 \mathrm{MHz}, \mathrm{CDCl}_{3}\right): \delta 7.88(\mathrm{~d}, J=8.8 \mathrm{~Hz}, 2 \mathrm{H}), 7.43(\mathrm{~d}, J=8.8 \mathrm{~Hz}, 2 \mathrm{H}), 5.05(\mathrm{t}, J=4.8$ $\mathrm{Hz}, 1 \mathrm{H}), 4.02-4.30(\mathrm{~m}, 2 \mathrm{H}), 3.42$ (sextet, $J=6.8 \mathrm{~Hz}, 1 \mathrm{H}), 2.28$ (t, $J=7.2 \mathrm{~Hz}, 2 \mathrm{H}$ ), 2.00-2.20 (m, 2H), 1.87-1.95 (m, 4H), 1.76-1.87 (m, 1H), 1.55-1.71 (m, 4H), $1.40-1.52(\mathrm{~m}, 10 \mathrm{H}), 1.19(\mathrm{~d}, J=8.4 \mathrm{~Hz}, 3 \mathrm{H}) \mathrm{ppm} ;{ }^{13} \mathrm{C} \mathrm{NMR}\left(101 \mathrm{MHz}, \mathrm{CDCl}_{3}\right): \delta$ 202.6, 172.3, 153.3, 139.4, 134.7, 129.6, 129.0, 79.3, 67.8, 52.0-52.4 (m, 1C), 40.4, 35.2-35.6 (m, 1C), 34.8, 32.8, 28.4, 27.7-28.1 (m, 1C), 22.7, 17.4 ppm; ESI-MS: $m / z$ $486.2\left(\mathrm{M}^{+}+\mathrm{Na}\right)$; HRMS calcd for $\mathrm{C}_{25} \mathrm{H}_{34} \mathrm{ClNNaO}_{5}[\mathrm{M}+\mathrm{Na}]$ : 486.2018, found 486.2013.

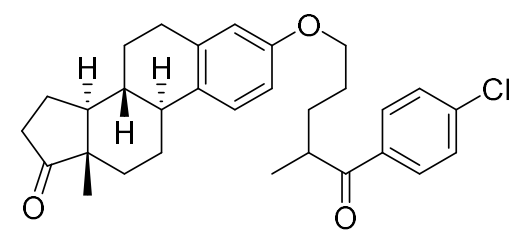

(8R,9S,13S,14S)-3-((5-(4-chlorophenyl)-4-methyl-5-oxopentyl)oxy)-13-methyl6,7,8,9,11,12,13,14,15,16-decahydro-17H-cyclopenta[a]phenanthren-17-one (4zf). This compound was isolated as a 50:50 mixture of two stereoisomers determined by ${ }^{1} \mathrm{H}$ NMR (400 MHz). Colorless oil; IR (neat): $v\left(\mathrm{~cm}^{-1}\right)$ 2930, 2868, 1737, 1682, 1609, 1589 , 1499, 1234, 1092, 974, 733; ${ }^{1} \mathrm{H}$ NMR (400 MHz, $\left.\mathrm{CDCl}_{3}\right): \delta 7.90$ (d, $\left.J=8.8 \mathrm{~Hz}, 2 \mathrm{H}\right)$, $7.43(\mathrm{~d}, J=8.8 \mathrm{~Hz}, 2 \mathrm{H}), 7.18(\mathrm{~d}, J=8.4 \mathrm{~Hz}, 1 \mathrm{H}), 6.64 / 6.63(2 \mathrm{~d}, J=8.4 \mathrm{~Hz}, 1 \mathrm{H}), 6.57$ (s, 1H), $3.93(\mathrm{t}, J=6.8 \mathrm{~Hz}, 2 \mathrm{H}), 3.51$ (sextet, $J=6.8 \mathrm{~Hz}, 1 \mathrm{H}), 2.83-2.91(\mathrm{~m}, 2 \mathrm{H}), 2.50$ (dd, $J=19.2,8.8 \mathrm{~Hz}, 1 \mathrm{H}), 2.36-2.44(\mathrm{~m}, 1 \mathrm{H}), 2.20-2.29(\mathrm{~m}, 1 \mathrm{H}), 1.91-2.19(\mathrm{~m}, 5 \mathrm{H})$, $1.72-1.86(\mathrm{~m}, 2 \mathrm{H}), 1.36-1.68(\mathrm{~m}, 7 \mathrm{H}), 1.22(\mathrm{~d}, J=6.8 \mathrm{~Hz}, 3 \mathrm{H}), 0.91(\mathrm{~s}, 3 \mathrm{H}) \mathrm{ppm} ;{ }^{13} \mathrm{C}$ NMR (101 MHz, $\left.\mathrm{CDCl}_{3}\right): \delta 220.9,202.8,156.8,139.3,137.7,134.8,132.0,129.7$, $128.9,126.3,114.5,112.0 / 111.9,67.5 / 67.4,50.4,48.0,43.9,40.2,38.3,35.8,31.5,30.0$, 29.6, 26.9, 26.5, 25.9, 21.5, 17.4, 13.8 ppm; ESI-MS: $m / z$ 501.3 $\left(\mathrm{M}^{+}+\mathrm{Na}\right)$; HRMS calcd for $\mathrm{C}_{30} \mathrm{H}_{36} \mathrm{ClO}_{3}[\mathrm{M}+\mathrm{H}]$ : 479.2347, found 479.2347 . 
<smiles>CC(CCc1ccccc1)C(=O)c1ccccc1</smiles>

2-Methyl-1,4-diphenylbutan-1-one (6a). Colorless oil; IR (neat): $v\left(\mathrm{~cm}^{-1}\right)$ 3061, 3026, 2968, 2931, 1682, 1596, 1495, 1448, 1376, 1226, 974, 670; ${ }^{1} \mathrm{H}$ NMR (400 MHz, $\left.\mathrm{CDCl}_{3}\right): \delta 7.87(\mathrm{~d}, J=8.4 \mathrm{~Hz}, 2 \mathrm{H}), 7.55(\mathrm{t}, J=7.2 \mathrm{~Hz}, 1 \mathrm{H}), 7.44(\mathrm{t}, J=8.0 \mathrm{~Hz}, 2 \mathrm{H})$, $7.28(\mathrm{t}, J=7.2 \mathrm{~Hz}, 2 \mathrm{H}), 7.20(\mathrm{t}, J=7.2 \mathrm{~Hz}, 1 \mathrm{H}), 7.16(\mathrm{~d}, J=7.2 \mathrm{~Hz}, 2 \mathrm{H}), 3.48$ (sextet, $J=6.4 \mathrm{~Hz}, 1 \mathrm{H}), 2.66(\mathrm{t}, J=8.0 \mathrm{~Hz}, 2 \mathrm{H}), 2.13-2.24(\mathrm{~m}, 1 \mathrm{H}), 1.70-1.81(\mathrm{~m}, 1 \mathrm{H}), 1.24$ $(\mathrm{d}, J=6.8 \mathrm{~Hz}, 3 \mathrm{H}) \mathrm{ppm} ;{ }^{13} \mathrm{C} \mathrm{NMR}\left(101 \mathrm{MHz}, \mathrm{CDCl}_{3}\right): \delta 204.1,141.7,136.5,132.9$, 128.6, 128.5, 128.4, 128.2, 125.9, 39.7, 35.1, 33.4, 17.3 ppm; ESI-MS: $m / z 239.1$ $\left(\mathrm{M}^{+}+\mathrm{H}\right)$; HRMS calcd for $\mathrm{C}_{17} \mathrm{H}_{19} \mathrm{O}[\mathrm{M}+\mathrm{H}]: 239.1430$, found 239.1430.

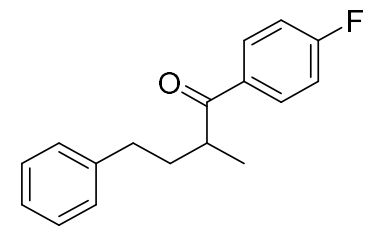

1-(4-Fluorophenyl)-2-methyl-4-phenylbutan-1-one (6b). Colorless oil; IR (neat): v $\left(\mathrm{cm}^{-1}\right)$ 3026, 1683, 1597, 1506, 1453, 1227, 1156, 976, 848, 749; ${ }^{1} \mathrm{H}$ NMR (400 MHz, $\left.\mathrm{CDCl}_{3}\right): \delta 7.84-7.90(\mathrm{~m}, 2 \mathrm{H}), 7.28(\mathrm{t}, J=7.2 \mathrm{~Hz}, 2 \mathrm{H}), 7.20(\mathrm{t}, J=7.2 \mathrm{~Hz}, 1 \mathrm{H}), 7.15(\mathrm{~d}$, $J=7.2 \mathrm{~Hz}, 2 \mathrm{H}), 7.10$ (t, $J=8.4 \mathrm{~Hz}, 2 \mathrm{H}), 3.41$ (sextet, $J=6.8 \mathrm{~Hz}, 1 \mathrm{H}), 2.65$ (t, $J=7.6$ $\mathrm{Hz}, 2 \mathrm{H}), 2.10-2.21(\mathrm{~m}, 1 \mathrm{H}), 1.70-1.80(\mathrm{~m}, 1 \mathrm{H}), 1.23(\mathrm{~d}, J=6.8 \mathrm{~Hz}, 3 \mathrm{H}) \mathrm{ppm} ;{ }^{19} \mathrm{~F}$ $\operatorname{NMR}\left(376 \mathrm{MHz}, \mathrm{CDCl}_{3}\right): \delta-105.7 \mathrm{ppm} ;{ }^{13} \mathrm{C} \mathrm{NMR}\left(101 \mathrm{MHz}, \mathrm{CDCl}_{3}\right): \delta 202.4,165.6$ $(\mathrm{d}, J=256.0 \mathrm{~Hz}), 141.6,132.8(\mathrm{~d}, J=3.1 \mathrm{~Hz}), 130.9$ (d, $J=9.2 \mathrm{~Hz}), 128.5,128.4$, 126.0, $115.6(\mathrm{~d}, J=21.4 \mathrm{~Hz}), 39.5,35.1,33.4,17.2 \mathrm{ppm}$; ESI-MS: $m / z 257.1\left(\mathrm{M}^{+}+\mathrm{H}\right)$; HRMS calcd for $\mathrm{C}_{17} \mathrm{H}_{18} \mathrm{FO}[\mathrm{M}+\mathrm{H}]: 257.1336$, found 257.1335 .

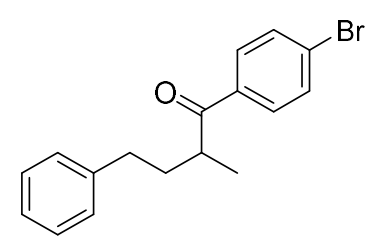


1-(4-Bromophenyl)-2-methyl-4-phenylbutan-1-one (6c). Colorless oil; IR (neat): v $\left(\mathrm{cm}^{-1}\right)$ 3061, 3026, 2968, 2932, 1683, 1584, 1454, 1396, 1219, 1071, 974, 840, 750; ${ }^{1} \mathrm{H}$ NMR (400 MHz, $\left.\mathrm{CDCl}_{3}\right): \delta 7.70(\mathrm{~d}, J=8.0 \mathrm{~Hz}, 2 \mathrm{H}), 7.57(\mathrm{~d}, J=8.0 \mathrm{~Hz}, 2 \mathrm{H}), 7.28(\mathrm{t}$, $J=7.6 \mathrm{~Hz}, 2 \mathrm{H}), 7.20(\mathrm{t}, J=7.2 \mathrm{~Hz}, 1 \mathrm{H}), 7.14$ (d, $J=7.6 \mathrm{~Hz}, 2 \mathrm{H}), 3.39$ (sextet, $J=6.4$ $\mathrm{Hz}, 1 \mathrm{H}), 2.64(\mathrm{t}, J=7.6 \mathrm{~Hz}, 2 \mathrm{H}), 2.09-2.21(\mathrm{~m}, 1 \mathrm{H}), 1.69-1.80(\mathrm{~m}, 1 \mathrm{H}), 1.23(\mathrm{~d}, J=$ $7.2 \mathrm{~Hz}, 3 \mathrm{H}) \mathrm{ppm} ;{ }^{13} \mathrm{C} \mathrm{NMR}\left(101 \mathrm{MHz}, \mathrm{CDCl}_{3}\right): \delta 203.0,141.5,135.1,131.9,129.8$, 128.5, 128.4, 128.0, 126.0, 39.6, 35.0, 33.3, 17.1 ppm; ESI-MS: $m / z$ 317.1/319.1 $\left(\mathrm{M}^{+}+\mathrm{H}\right)$; HRMS calcd for $\mathrm{C}_{17} \mathrm{H}_{18} \mathrm{BrO}[\mathrm{M}+\mathrm{H}]:$ 317.0536, found 317.0536.

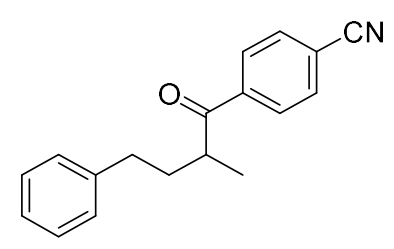

4-(2-Methyl-4-phenylbutanoyl)benzonitrile (6d). Colorless oil; IR (neat): $v\left(\mathrm{~cm}^{-1}\right)$ $3062,3026,2971,2934,2230,1687,1604,1496,1454,1405,1220,977,852,753 ;{ }^{1} \mathrm{H}$ NMR (400 MHz, $\left.\mathrm{CDCl}_{3}\right): \delta 7.87(\mathrm{~d}, J=8.4 \mathrm{~Hz}, 2 \mathrm{H}), 7.72(\mathrm{~d}, J=8.4 \mathrm{~Hz}, 2 \mathrm{H}), 7.28$ (t, $J=7.2 \mathrm{~Hz}, 2 \mathrm{H}), 7.21(\mathrm{t}, J=7.2 \mathrm{~Hz}, 1 \mathrm{H}), 7.13(\mathrm{~d}, J=7.2 \mathrm{~Hz}, 2 \mathrm{H}), 3.40$ (sextet, $J=6.4$ $\mathrm{Hz}, 1 \mathrm{H}), 2.66(\mathrm{t}, J=7.6 \mathrm{~Hz}, 2 \mathrm{H}), 2.09-2.20(\mathrm{~m}, 1 \mathrm{H}), 1.71-1.82(\mathrm{~m}, 1 \mathrm{H}), 1.24(\mathrm{~d}, J=$ $6.8 \mathrm{~Hz}, 3 \mathrm{H}) \mathrm{ppm} ;{ }^{13} \mathrm{C} \mathrm{NMR}\left(101 \mathrm{MHz}, \mathrm{CDCl}_{3}\right): \delta 202.6,141.2,139.4,132.4,128.6$, 128.5, 126.1, 117.9, 116.1, 39.9, 34.8, 33.2, 16.8 ppm; ESI-MS: $m / z 264.1\left(\mathrm{M}^{+}+\mathrm{H}\right)$; HRMS calcd for $\mathrm{C}_{18} \mathrm{H}_{18} \mathrm{NO}[\mathrm{M}+\mathrm{H}]: 264.1383$, found 264.1384 .

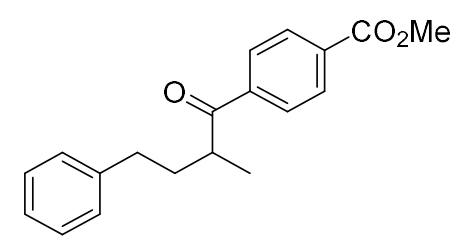

Methyl 4-(2-methyl-4-phenylbutanoyl)benzoate (6e). Colorless oil; IR (neat): $v$ ( $\mathrm{cm}^{-}$ 1) $3068,3026,2950,1726,1685,1497,1454,1436,1279,1107,977,722 ;{ }^{1} \mathrm{H}$ NMR $\left(400 \mathrm{MHz}, \mathrm{CDCl}_{3}\right): \delta 8.09(\mathrm{~d}, J=8.8 \mathrm{~Hz}, 2 \mathrm{H}), 7.87(\mathrm{~d}, J=8.8 \mathrm{~Hz}, 2 \mathrm{H}), 7.28(\mathrm{t}, J=7.2$ $\mathrm{Hz}, 2 \mathrm{H}), 7.20$ (t, $J=7.2 \mathrm{~Hz}, 1 \mathrm{H}), 7.14$ (d, $J=7.2 \mathrm{~Hz}, 2 \mathrm{H}), 3.95$ (s, 3H), 3.45 (sextet, $J$ $=6.4 \mathrm{~Hz}, 1 \mathrm{H}), 2.66(\mathrm{t}, J=7.6 \mathrm{~Hz}, 2 \mathrm{H}), 2.11-2.21(\mathrm{~m}, 1 \mathrm{H}), 1.70-1.81(\mathrm{~m}, 1 \mathrm{H}), 1.24(\mathrm{~d}$, 
$J=6.8 \mathrm{~Hz}, 3 \mathrm{H}) \mathrm{ppm} ;{ }^{13} \mathrm{C} \mathrm{NMR}\left(101 \mathrm{MHz}, \mathrm{CDCl}_{3}\right): \delta 203.5,166.2,141.5,139.8,133.6$, 129.8, 128.5, 128.4, 128.1, 126.0, 52.4, 40.0, 35.0, 33.3, 17.0 ppm; ESI-MS: $m / z 297.1$ $\left(\mathrm{M}^{+}+\mathrm{H}\right)$; HRMS calcd for $\mathrm{C}_{19} \mathrm{H}_{21} \mathrm{O}_{3}[\mathrm{M}+\mathrm{H}]: 297.1485$, found 297.1484.

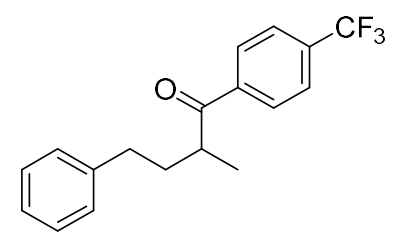

2-Methyl-4-phenyl-1-(4-(trifluoromethyl)phenyl)butan-1-one (6f). Colorless oil; IR (neat): $v\left(\mathrm{~cm}^{-1}\right)$ 3064, 3027, 2971, 2934, 1689, 1510, 1455, 1410, 1325, 1170, 1130 , 1067, 977, 855; ${ }^{1} \mathrm{H}$ NMR (400 MHz, $\left.\mathrm{CDCl}_{3}\right): \delta 7.92(\mathrm{~d}, J=8.4 \mathrm{~Hz}, 2 \mathrm{H}), 7.69$ (d, $J=$ $8.4 \mathrm{~Hz}, 2 \mathrm{H}), 7.29$ (t, $J=7.2 \mathrm{~Hz}, 2 \mathrm{H}), 7.21$ (t, $J=7.2 \mathrm{~Hz}, 1 \mathrm{H}), 7.15$ (d, $J=6.8 \mathrm{~Hz}, 2 \mathrm{H})$, 3.44 (sextet, $J=6.4 \mathrm{~Hz}, 1 \mathrm{H}), 2.67$ (t, $J=7.6 \mathrm{~Hz}, 2 \mathrm{H}), 2.12-2.23(\mathrm{~m}, 1 \mathrm{H}), 1.72-1.82$ $(\mathrm{m}, 1 \mathrm{H}), 1.25(\mathrm{~d}, J=7.2 \mathrm{~Hz}, 3 \mathrm{H}) \mathrm{ppm} ;{ }^{19} \mathrm{~F} \mathrm{NMR}\left(376 \mathrm{MHz}, \mathrm{CDCl}_{3}\right): \delta-63.1 \mathrm{ppm} ;{ }^{13} \mathrm{C}$ NMR (101 MHz, $\left.\mathrm{CDCl}_{3}\right): \delta 203.0,141.4,139.2,134.1$ (q, $\left.J=32.9 \mathrm{~Hz}\right), 128.6,128.5$, 126.1, $125.6(\mathrm{q}, J=3.8 \mathrm{~Hz}), 123.6(\mathrm{q}, J=273.8 \mathrm{~Hz}), 40.0,34.9,33.3,16.9$ ppm; ESIMS: $m / z 307.1\left(\mathrm{M}^{+}+\mathrm{H}\right)$; HRMS calcd for $\mathrm{C}_{18} \mathrm{H}_{18} \mathrm{~F}_{3} \mathrm{O}[\mathrm{M}+\mathrm{H}]: 307.1304$, found 307.1303 .

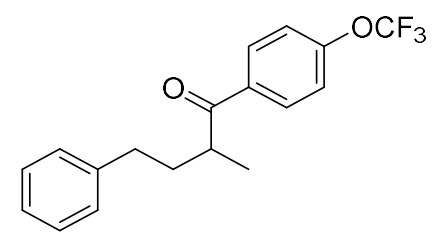

2-Methyl-4-phenyl-1-(4-(trifluoromethoxy)phenyl)butan-1-one (6g). Colorless oil; IR (neat): $v\left(\mathrm{~cm}^{-1}\right) 3064,3028,2971,2935,1687,1603,1505,1454,1377,1259,1212$, 1167, 976, 859, 748; ${ }^{1} \mathrm{H}$ NMR (400 MHz, $\left.\mathrm{CDCl}_{3}\right): \delta 7.88$ (d, J=8.4 Hz, 2H), 7.23-7.32 (m, 4H), $7.20(\mathrm{t}, J=7.2 \mathrm{~Hz}, 1 \mathrm{H}), 7.15(\mathrm{~d}, J=7.2 \mathrm{~Hz}, 2 \mathrm{H}), 3.41$ (sextet, $J=6.4 \mathrm{~Hz}, 1 \mathrm{H}$ ), $2.65(\mathrm{t}, J=7.6 \mathrm{~Hz}, 2 \mathrm{H}), 2.11-2.22(\mathrm{~m}, 1 \mathrm{H}), 1.70-1.81(\mathrm{~m}, 1 \mathrm{H}), 1.24(\mathrm{~d}, J=6.4 \mathrm{~Hz}, 3 \mathrm{H})$ ppm; ${ }^{19} \mathrm{~F} \mathrm{NMR}\left(376 \mathrm{MHz}, \mathrm{CDCl}_{3}\right): \delta-57.6 \mathrm{ppm} ;{ }^{13} \mathrm{C} \mathrm{NMR}\left(101 \mathrm{MHz}, \mathrm{CDCl}_{3}\right.$ ): $\delta 202.4$, $152.4(\mathrm{~d}, J=1.5 \mathrm{~Hz}), 141.5,134.7,130.2,128.5,128.4,126.0,120.4,120.3$ (q, $J=$ 260.0 Hz), 39.7, 35.0, 33.4, 17.1 ppm; ESI-MS: $m / z$ 323.1 $\left(\mathrm{M}^{+}+\mathrm{H}\right)$; HRMS calcd for $\mathrm{C}_{18} \mathrm{H}_{18} \mathrm{~F}_{3} \mathrm{O}_{2}[\mathrm{M}+\mathrm{H}]:$ 323.1253, found 323.1249. 


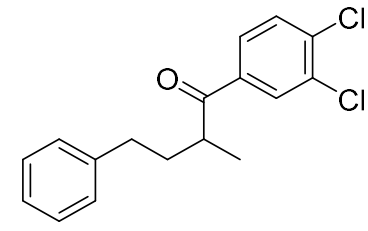

1-(3,4-Dichlorophenyl)-2-methyl-4-phenylbutan-1-one (6h). Colorless oil; IR (neat): $v\left(\mathrm{~cm}^{-1}\right) 3063,3026,2970,2933,1688,1584,1556,1455,1377,1213,1030,986,748$; ${ }^{1} \mathrm{H}$ NMR (400 MHz, $\left.\mathrm{CDCl}_{3}\right): \delta 7.90(\mathrm{~d}, J=8.4 \mathrm{~Hz}, 1 \mathrm{H}), 7.63(\mathrm{dd}, J=8.4,2.0 \mathrm{~Hz}, 1 \mathrm{H})$, $7.50(\mathrm{~d}, J=8.4 \mathrm{~Hz}, 1 \mathrm{H}), 7.29(\mathrm{t}, J=7.2 \mathrm{~Hz}, 2 \mathrm{H}), 7.21(\mathrm{t}, J=7.6 \mathrm{~Hz}, 1 \mathrm{H}), 7.14(\mathrm{~d}, J=$ $6.8 \mathrm{~Hz}, 2 \mathrm{H}), 3.34$ (sextet, $J=6.8 \mathrm{~Hz}, 1 \mathrm{H}), 2.65$ (t, $J=7.6 \mathrm{~Hz}, 2 \mathrm{H}), 2.09-2.20(\mathrm{~m}, 1 \mathrm{H})$, $1.70-1.80(\mathrm{~m}, 1 \mathrm{H}), 1.23(\mathrm{~d}, J=6.8 \mathrm{~Hz}, 3 \mathrm{H}) \mathrm{ppm} ;{ }^{13} \mathrm{C} \mathrm{NMR}\left(101 \mathrm{MHz}, \mathrm{CDCl}_{3}\right): \delta 201.7$, $141.3,137.4,136.0,133.3,130.7,130.3,128.5,128.4,127.3,126.1,39.6,34.9,33.2$, 17.0 ppm; ESI-MS: $m / z$ 307.1( $\left.\mathrm{M}^{+}+\mathrm{H}\right)$; HRMS calcd for $\mathrm{C}_{17} \mathrm{H}_{17} \mathrm{Cl}_{2} \mathrm{O}[\mathrm{M}+\mathrm{H}]$ : 307.0651 , found 307.0650 .

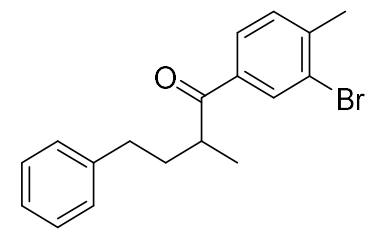

1-(3-Bromo-4-methylphenyl)-2-methyl-4-phenylbutan-1-one (6i). Colorless oil; IR (neat): $v\left(\mathrm{~cm}^{-1}\right) 3061,3026,2969,2931,1683,1597,1554,1454,1382,1216,1038$, 748; ${ }^{1} \mathrm{H}$ NMR (400 MHz, $\left.\mathrm{CDCl}_{3}\right): \delta 8.02(\mathrm{~d}, J=2.0 \mathrm{~Hz}, 1 \mathrm{H}), 7.67(\mathrm{dd}, J=8.4 \mathrm{~Hz}, 1 \mathrm{H})$, $7.25-7.32(\mathrm{~m}, 3 \mathrm{H}), 7.20(\mathrm{t}, J=7.2 \mathrm{~Hz}, 1 \mathrm{H}), 7.15(\mathrm{~d}, J=6.8 \mathrm{~Hz}, 2 \mathrm{H}), 3.38$ (sextet, $J=$ $6.4 \mathrm{~Hz}, 1 \mathrm{H}), 2.64(\mathrm{t}, J=7.6 \mathrm{~Hz}, 2 \mathrm{H}), 2.45(\mathrm{~s}, 3 \mathrm{H}), 2.10-2.21(\mathrm{~m}, 1 \mathrm{H}), 1.68-1.79(\mathrm{~m}$, $1 \mathrm{H}), 1.22(\mathrm{~d}, J=6.8 \mathrm{~Hz}, 3 \mathrm{H}) \mathrm{ppm} ;{ }^{13} \mathrm{C} \mathrm{NMR}\left(101 \mathrm{MHz}, \mathrm{CDCl}_{3}\right): \delta 202.4,143.3,141.5$, $135.9,132.3,130.9,128.5,128.4,127.0,126.0,125.3,39.6,35.1,33.3,23.1,17.2$ ppm; ESI-MS: $m / z$ 331.1/333.1 $\left(\mathrm{M}^{+}+\mathrm{H}\right)$; HRMS calcd for $\mathrm{C}_{18} \mathrm{H}_{20} \mathrm{BrO}[\mathrm{M}+\mathrm{H}]$ : 331.0692, found 331.0690 . 


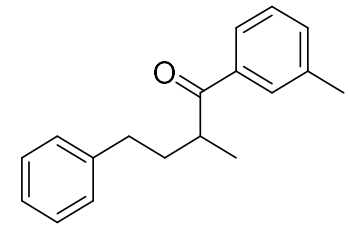

2-Methyl-4-phenyl-1-(m-tolyl)butan-1-one (6j). Colorless oil; IR (neat): $v\left(\mathrm{~cm}^{-1}\right)$ 3061, 3026, 2967, 2931, 1682, 1603, 1585, 1454, 1254, 1158, 740; ${ }^{1} \mathrm{H}$ NMR (400 MHz, $\left.\mathrm{CDCl}_{3}\right): \delta 7.63-7.69(\mathrm{~m}, 2 \mathrm{H}), 7.24-7.39(\mathrm{~m}, 4 \mathrm{H}), 7.20(\mathrm{t}, J=7.2 \mathrm{~Hz}, 1 \mathrm{H}), 7.16(\mathrm{~d}, J=$ $7.2 \mathrm{~Hz}, 2 \mathrm{H}), 3.46$ (sextet, $J=6.8 \mathrm{~Hz}, 1 \mathrm{H}), 2.65$ (t, $J=7.2 \mathrm{~Hz}, 2 \mathrm{H}), 2.39$ (s, 3H), 2.11-2.22 (m, 1H), 1.69-1.80 (m, 1H), $1.23(\mathrm{~d}, J=7.2 \mathrm{~Hz}, 3 \mathrm{H}) \mathrm{ppm} ;{ }^{13} \mathrm{C}$ NMR $(101$ $\left.\mathrm{MHz}, \mathrm{CDCl}_{3}\right): \delta 204.3,141.8,138.4,136.6,133.6,128.8,128.5,128.4,128.3,125.9$, 125.4, 39.6, 35.2, 33.4, 21.3, 17.2 ppm; ESI-MS: $m / z$ 253.2 $\left(\mathrm{M}^{+}+\mathrm{H}\right)$; HRMS calcd for $\mathrm{C}_{18} \mathrm{H}_{21} \mathrm{O}[\mathrm{M}+\mathrm{H}]: 253.1587$, found 253.1584.<smiles>COc1cccc(C(=O)C(C)CCc2ccccc2)c1</smiles>

1-(3-Methoxyphenyl)-2-methyl-4-phenylbutan-1-one (6k). Colorless oil; IR (neat): $v\left(\mathrm{~cm}^{-1}\right) 3062,3026,2974,2935,1682,1596,1582,1454,1428,1261,1045,747 ;{ }^{1} \mathrm{H}$ NMR (400 MHz, $\left.\mathrm{CDCl}_{3}\right): \delta 7.41-7.45(\mathrm{~m}, 2 \mathrm{H}), 7.34(\mathrm{t}, J=8.0 \mathrm{~Hz}, 1 \mathrm{H}), 7.27(\mathrm{t}, J=7.2$ Hz, 2H), 7.20 (t, $J=7.2 \mathrm{~Hz}, 1 \mathrm{H}), 7.15$ (d, $J=6.8 \mathrm{~Hz}, 2 \mathrm{H}), 7.07-7.12(\mathrm{~m}, 1 \mathrm{H}), 3.84$ (s, $3 \mathrm{H}), 3.45$ (sextet, $J=6.8 \mathrm{~Hz}, 1 \mathrm{H}), 2.64(\mathrm{t}, J=6.8 \mathrm{~Hz}, 2 \mathrm{H}), 2.12-2.23(\mathrm{~m}, 1 \mathrm{H})$, 1.69-1.81 (m, 1H), $1.24(\mathrm{~d}, J=6.4 \mathrm{~Hz}, 3 \mathrm{H}) \mathrm{ppm} ;{ }^{13} \mathrm{C} \mathrm{NMR}\left(101 \mathrm{MHz}, \mathrm{CDCl}_{3}\right): \delta 203.9$, $159.9,141.7,137.9,129.5,128.5,128.4,125.9,120.8,119.4 .112 .5,55.4,39.9,35.2$, 33.4, 17.3 ppm; ESI-MS: $m / z 269.2\left(\mathrm{M}^{+}+\mathrm{H}\right)$; HRMS calcd for $\mathrm{C}_{18} \mathrm{H}_{21} \mathrm{O}_{2}[\mathrm{M}+\mathrm{H}]$ : 269.1536, found 269.1533.

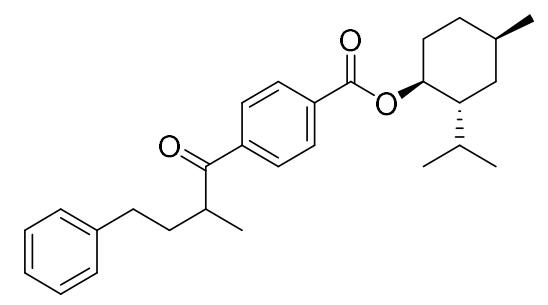


benzoate (61). This compound was isolated as a 50:50 mixture of two stereoisomers determined by ${ }^{1} \mathrm{H}$ NMR (400 MHz). Colorless oil; IR (neat): $v\left(\mathrm{~cm}^{-1}\right)$ 3026, 2955, 2930, 2869, 1717, 1686, 1455, 1275, 1116, 977, 722; ${ }^{1} \mathrm{H}$ NMR (400 MHz, $\left.\mathrm{CDCl}_{3}\right): \delta 8.09$ (d, $J=8.0 \mathrm{~Hz}, 2 \mathrm{H}), 7.88(\mathrm{~d}, J=8.0 \mathrm{~Hz}, 2 \mathrm{H}), 7.28(\mathrm{t}, J=7.2 \mathrm{~Hz}, 2 \mathrm{H}), 7.20(\mathrm{t}, J=7.2 \mathrm{~Hz}$, 1H), $7.14(\mathrm{~d}, J=7.2 \mathrm{~Hz}, 2 \mathrm{H}), 4.96(\mathrm{td}, J=11.2,4.4 \mathrm{~Hz}, 1 \mathrm{H}), 3.45$ (sextet, $J=6.8 \mathrm{~Hz}$, $1 \mathrm{H}), 2.65(\mathrm{t}, J=7.6 \mathrm{~Hz}, 2 \mathrm{H}), 2.09-2.24(\mathrm{~m}, 2 \mathrm{H}), 1.89-2.00(\mathrm{~m}, 1 \mathrm{H}), 1.70-1.82(\mathrm{~m}$, $3 \mathrm{H}), 1.50-1.63(\mathrm{~m}, 2 \mathrm{H}), 1.24(\mathrm{~d}, J=6.8 \mathrm{~Hz}, 3 \mathrm{H}), 1.06-1.21(\mathrm{~m}, 2 \mathrm{H}), 0.88-1.00(\mathrm{~m}$, $7 \mathrm{H}), 0.80(\mathrm{~d}, J=7.2 \mathrm{~Hz}, 3 \mathrm{H}) \mathrm{ppm} ;{ }^{13} \mathrm{C} \mathrm{NMR}\left(101 \mathrm{MHz}, \mathrm{CDCl}_{3}\right): \delta 203.5,165.2$, 141.5/141.4, 139.6, 134.3, 129.8, 128.4, 128.3, 128.1, 126.0, 75.4, 47.2, 40.8, 40.0/39.9, 34.9, 34.2, 33.3, 31.4, 26.5, 23.6, 22.0, 20.7, 17.0, 16.5 ppm; ESI-MS: $m / z 443.3$ $\left(\mathrm{M}^{+}+\mathrm{Na}\right)$; HRMS calcd for $\mathrm{C}_{28} \mathrm{H}_{36} \mathrm{NaO}_{3}[\mathrm{M}+\mathrm{Na}]: 443.2557$, found 443.2551.

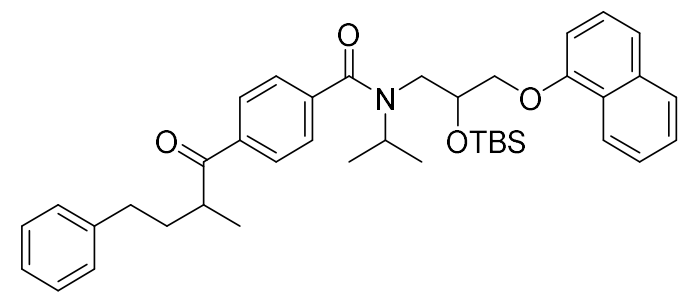

$\mathrm{N}$-(2-((tert-butyldimethylsilyl)oxy)-3-(naphthalen-1-yloxy)propyl)- $\mathrm{N}$-isopropyl-4(2-methyl-4-phenylbutanoyl)benzamide (6m). This compound was isolated as a 50:50 mixture of two stereoisomers determined by ${ }^{13} \mathrm{C}$ NMR $(101 \mathrm{MHz})$. Colorless oil; IR (neat): $v\left(\mathrm{~cm}^{-1}\right) 3055,2930,2856,1683,1636,1580,1461,1403,1268,1240,1102$, 976, 836, 773; ${ }^{1} \mathrm{H}$ NMR (400 MHz, $\left.\mathrm{CDCl}_{3}\right): \delta 8.37$ (d, $\left.J=6.8 \mathrm{~Hz}, 1 \mathrm{H}\right), 7.87$ (d, $J=8.0$ $\mathrm{Hz}, 2 \mathrm{H}), 7.81(\mathrm{~d}, J=6.8 \mathrm{~Hz}, 1 \mathrm{H}), 7.42-7.54(\mathrm{~m}, 5 \mathrm{H}), 7.39$ (t, $J=7.6 \mathrm{~Hz}, 1 \mathrm{H}), 7.28$ (t, $J=7.6 \mathrm{~Hz}, 2 \mathrm{H}), 7.12-7.24(\mathrm{~m}, 3 \mathrm{H}), 6.85(\mathrm{~d}, J=7.2 \mathrm{~Hz}, 1 \mathrm{H}), 4.79-4.88(\mathrm{~m}, 1 \mathrm{H})$, 4.12-4.30 (m, 2H), 3.90-4.08 (m, 1H), $3.79(\mathrm{dd}, J=13.2,6.4 \mathrm{~Hz}, 1 \mathrm{H}), 3.38-3.54(\mathrm{~m}$, 2H), $2.66(\mathrm{t}, J=8.0 \mathrm{~Hz}, 2 \mathrm{H}), 2.10-2.25(\mathrm{~m}, 1 \mathrm{H}), 1.69-1.85(\mathrm{~m}, 1 \mathrm{H}), 1.20-1.35(\mathrm{~m}$, $6 \mathrm{H}), 1.13(\mathrm{~d}, J=6.0 \mathrm{~Hz}, 3 \mathrm{H}), 0.95(\mathrm{~s}, 9 \mathrm{H}), 0.24(\mathrm{~s}, 3 \mathrm{H}), 0.17(\mathrm{~s}, 3 \mathrm{H}) \mathrm{ppm} ;{ }^{13} \mathrm{C} \mathrm{NMR}$ $\left(101 \mathrm{MHz}, \mathrm{CDCl}_{3}\right): \delta 203.3,172.0 / 171.9,154.6,141.5,141.3 / 141.2,137.1 / 137.0,134.5$, $128.5,128.4,128.3,127.4,126.7,126.4,126.0,125.8,125.6,125.1,122.1,120.4,104.7$ $71.2,68.4,50.9,45.0,39.8,35.0,33.3,25.8,21.4,21.0,18.0,17.1,-4.3,-4.6$ ppm; ESI- 
MS: $m / z 638.4\left(\mathrm{M}^{+}+\mathrm{H}\right)$; HRMS calcd for $\mathrm{C}_{40} \mathrm{H}_{52} \mathrm{NO}_{4} \mathrm{Si}[\mathrm{M}+\mathrm{H}]: 638.3660$, found 638.3656 .

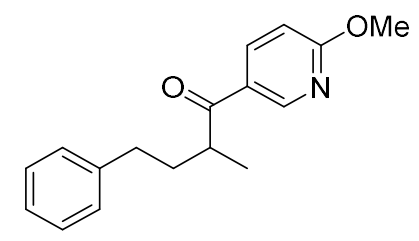

1-(6-Methoxypyridin-3-yl)-2-methyl-4-phenylbutan-1-one (6n). Colorless oil; IR (neat): $v\left(\mathrm{~cm}^{-1}\right) 3025,2946,1677,1600,1561,1495,1373,1295,1234,1117,1014$, 971; ${ }^{1} \mathrm{H}$ NMR (400 MHz, $\left.\mathrm{CDCl}_{3}\right): \delta 8.71(\mathrm{~s}, 1 \mathrm{H}), 8.07(\mathrm{dd}, J=8.8,2.0 \mathrm{~Hz}, 1 \mathrm{H}), 7.27$ (t, $J=6.8 \mathrm{~Hz}, 2 \mathrm{H}), 7.19(\mathrm{t}, J=6.8 \mathrm{~Hz}, 1 \mathrm{H}), 7.14(\mathrm{~d}, J=6.8 \mathrm{~Hz}, 2 \mathrm{H}), 6.77$ (d, $J=8.8$ $\mathrm{Hz}, 1 \mathrm{H}), 4.01(\mathrm{~s}, 3 \mathrm{H}), 3.36$ (sextet, $J=6.8 \mathrm{~Hz}, 1 \mathrm{H}), 2.64$ (t, $J=7.2 \mathrm{~Hz}, 2 \mathrm{H}), 2.11-2.22$ $(\mathrm{m}, 1 \mathrm{H}), 1.70-1.81(\mathrm{~m}, 1 \mathrm{H}), 1.23(\mathrm{~d}, J=6.4 \mathrm{~Hz}, 3 \mathrm{H}) \mathrm{ppm} ;{ }^{13} \mathrm{C} \mathrm{NMR}\left(101 \mathrm{MHz}, \mathrm{CDCl}_{3}\right)$ : $\delta 201.8,166.6,148.9,141.6,138.5,128.4,126.1,126.0,111.2,54.0,39.8,35.1,33.4$, 17.3 ppm; ESI-MS: $m / z 270.1\left(\mathrm{M}^{+}+\mathrm{H}\right)$; HRMS calcd for $\mathrm{C}_{17} \mathrm{H}_{20} \mathrm{NO}_{2}[\mathrm{M}+\mathrm{H}]: 270.1489$, found 270.1484 .

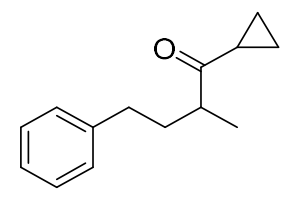

1-Cyclopropyl-2-methyl-4-phenylbutan-1-one (6o). Colorless oil; IR (neat): $v\left(\mathrm{~cm}^{-1}\right)$ 3027, 1696, 1497, 1453, 1385, 1054, 1016, 749; ${ }^{1} \mathrm{H}$ NMR (400 MHz, $\left.\mathrm{CDCl}_{3}\right): \delta 7.26$ (t, $J=7.6 \mathrm{~Hz}, 2 \mathrm{H}), 7.16-7.22(\mathrm{~m}, 3 \mathrm{H}), 2.70$ (sextet, $J=7.2 \mathrm{~Hz}, 1 \mathrm{H}), 2.61(\mathrm{t}, J=8.4 \mathrm{~Hz}$, 2H), 2.02-2.13 (m, 1H), 1.91-1.99 (m, 1H), 1.64-1.75 (m, 1H), 1.18 (d, J=6.8 Hz, $3 \mathrm{H}), 0.98-1.04(\mathrm{~m}, 2 \mathrm{H}), 0.83-0.89(\mathrm{~m}, 2 \mathrm{H}) \mathrm{ppm} ;{ }^{13} \mathrm{C} \mathrm{NMR}\left(101 \mathrm{MHz}, \mathrm{CDCl}_{3}\right): \delta 214.2$, 141.9, 128.4, 128.3, 125.9, 46.5, 34.6, 33.4, 19.1, 16.3, 10.8, 10.7 ppm; ESI-MS: $m / z$ $203.1\left(\mathrm{M}^{+}+\mathrm{H}\right)$; HRMS calcd for $\mathrm{C}_{14} \mathrm{H}_{19} \mathrm{O}[\mathrm{M}+\mathrm{H}]: 203.1430$, found 203.1431.

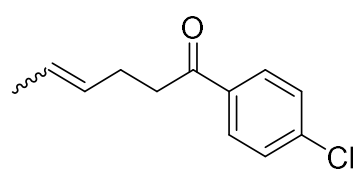


1-(4-Chlorophenyl)hex-4-en-1-one (8). This compound was isolated as a 66:34 mixture of two stereoisomers determined by ${ }^{1} \mathrm{H}$ NMR (400 MHz). Colorless oil; IR (neat): $v\left(\mathrm{~cm}^{-1}\right) 3018,2930,1688,1590,1487,1400,1201,1092,967 ;{ }^{1} \mathrm{H}$ NMR (400 $\left.\mathrm{MHz}_{\mathrm{CDCl}}\right): \delta 7.89 / 7.88(2 \mathrm{~d}, J=7.2 \mathrm{~Hz}, 2 \mathrm{H}), 7.14(\mathrm{~d}, J=8.4 \mathrm{~Hz}, 2 \mathrm{H}), 5.38-5.56(\mathrm{~m}$, 2H), $2.99(\mathrm{t}, J=7.6 \mathrm{~Hz}, 2 \mathrm{H}), 2.35-2.51(\mathrm{~m}, 2 \mathrm{H}), 1.61-1.67(\mathrm{~m}, 3 \mathrm{H}) \mathrm{ppm} ;{ }^{13} \mathrm{C} \mathrm{NMR}$ $\left(101 \mathrm{MHz}, \mathrm{CDCl}_{3}\right): \delta 198.5 / 198.4,139.3,135.3,129.5 / 129.4,128.9 / 128.6,126.1,125.3$, 38.5/38.3, 27.0/21.6, 17.9/12.7 ppm; ESI-MS: m/z 209.1 $\left(\mathrm{M}^{+}+\mathrm{H}\right)$; HRMS calcd for $\mathrm{C}_{12} \mathrm{H}_{14} \mathrm{ClO}[\mathrm{M}+\mathrm{H}]: 209.0728$, found 209.0726.

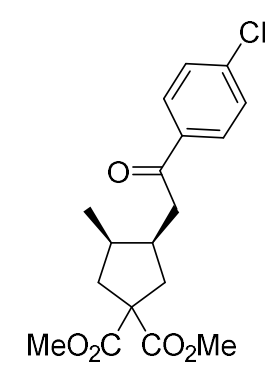

Dimethyl 3-(2-(4-chlorophenyl)-2-oxoethyl)-4-methylcyclopentane-1,1dicarboxylate (10). Colorless oil; IR (neat): $v\left(\mathrm{~cm}^{-1}\right)$ 2954, 1732, 1686, 1589, 1436, 1400, 1254, 1163, 1092, 818; ${ }^{1} \mathrm{H}$ NMR (400 MHz, $\left.\mathrm{CDCl}_{3}\right): \delta 7.88$ (d, $\left.J=8.4 \mathrm{~Hz}, 2 \mathrm{H}\right)$, $7.43(\mathrm{~d}, J=8.4 \mathrm{~Hz}, 2 \mathrm{H}), 3.72(\mathrm{~s}, 3 \mathrm{H}), 3.70(\mathrm{~s}, 3 \mathrm{H}), 2.98(\mathrm{dd}, J=16.8,6.4 \mathrm{~Hz}, 1 \mathrm{H}), 2.87$ $(\mathrm{dd}, J=16.8,8.0 \mathrm{~Hz}, 1 \mathrm{H}), 2.61-2.72(\mathrm{~m}, 1 \mathrm{H}), 2.45-2.56(\mathrm{~m}, 2 \mathrm{H}), 2.29-2.41(\mathrm{~m}, 1 \mathrm{H})$, $2.05(\mathrm{dd}, J=13.6,8.4 \mathrm{~Hz}, 1 \mathrm{H}), 1.98(\mathrm{dd}, J=13.6,6.4 \mathrm{~Hz}, 1 \mathrm{H}), 0.87(\mathrm{~d}, J=7.2 \mathrm{~Hz}, 3 \mathrm{H})$ ppm; ${ }^{13} \mathrm{C}$ NMR (101 MHz, $\left.\mathrm{CDCl}_{3}\right): \delta 198.2,173.3,173.1,139.4,135.4,129.4,128.9$, 58.7, 52.8, 52.7, 41.4, 38.9, 38.6, 38.0, 35.6, 15.3 ppm; ESI-MS: $m / z 353.1\left(\mathrm{M}^{+}+\mathrm{H}\right)$; HRMS calcd for $\mathrm{C}_{18} \mathrm{H}_{22} \mathrm{ClO}_{5}[\mathrm{M}+\mathrm{H}]: 353.1150$, found 353.1146 . 
5. ${ }^{1} \mathrm{H},{ }^{31} \mathrm{P},{ }^{13} \mathrm{C}$ and ${ }^{19} \mathrm{~F}$ NMR spectra of new substrates

\section{Compound 1za}

${ }^{1} \mathrm{H}$ NMR (400 MHz, $\left.\mathrm{CDCl}_{3}\right)$

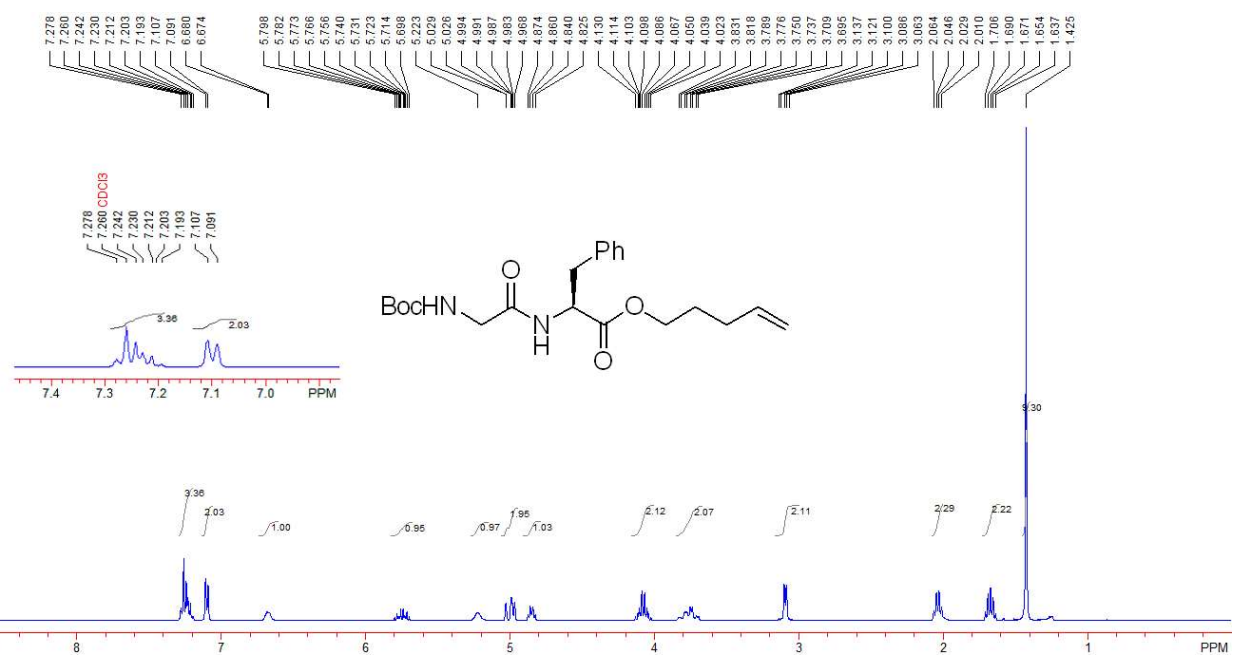

\section{Compound 1za}

${ }^{13} \mathrm{C} \mathrm{NMR}\left(101 \mathrm{MHz}, \mathrm{CDCl}_{3}\right)$

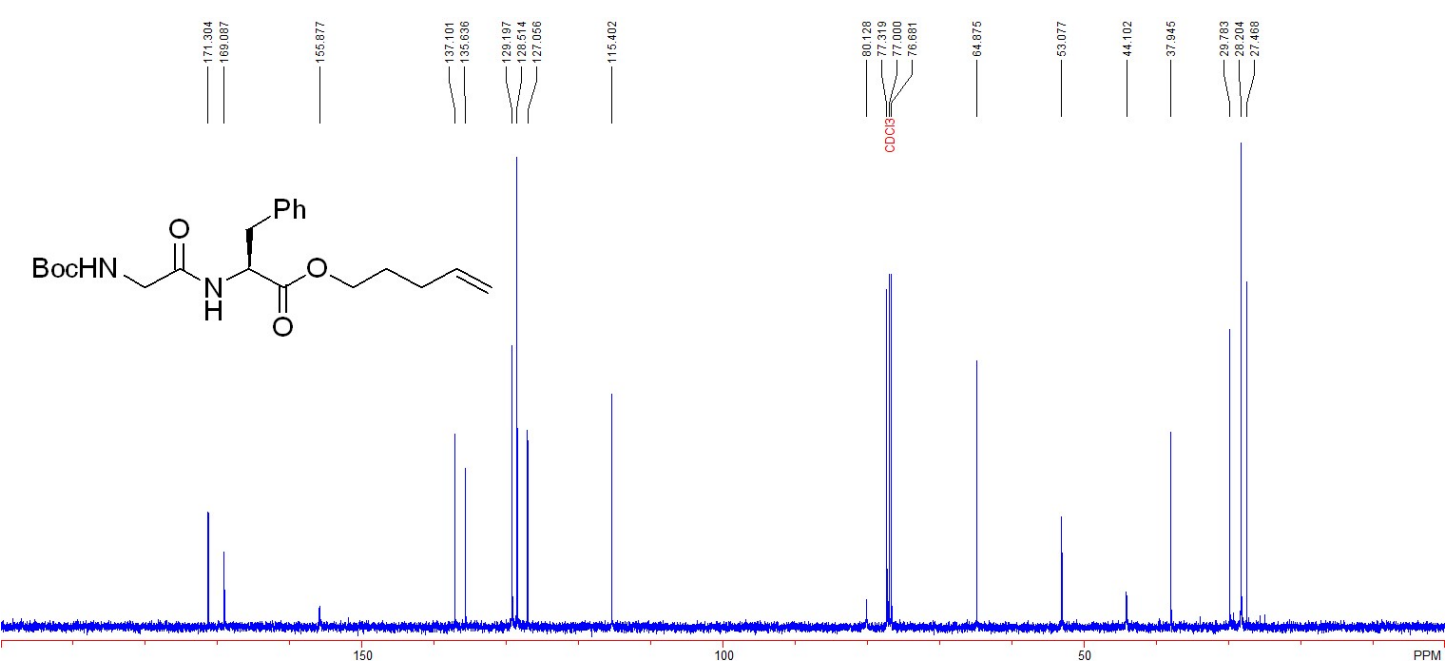




\section{Compound 1ze}

${ }^{1} \mathrm{H}$ NMR (400 MHz, $\mathrm{CDCl}_{3}$ )

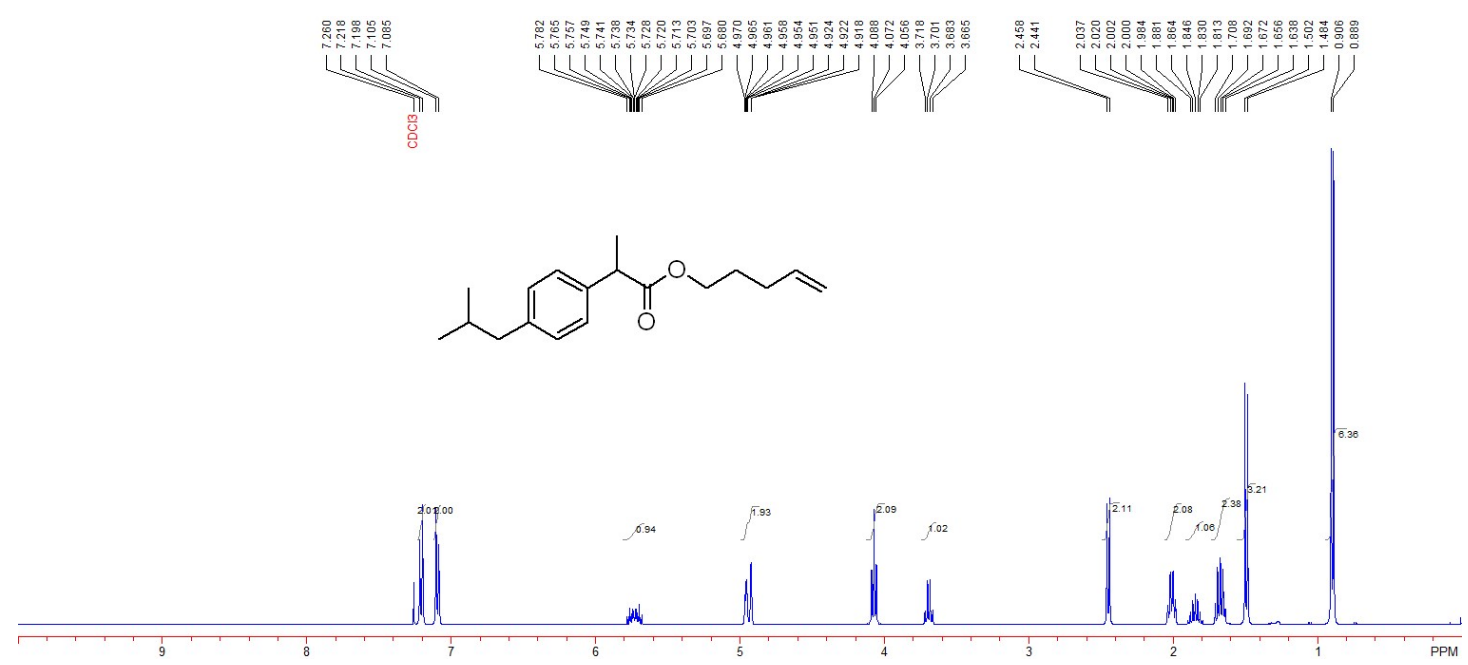

\section{Compound 1zc}

${ }^{13} \mathrm{C} \mathrm{NMR}\left(101 \mathrm{MHz}, \mathrm{CDCl}_{3}\right)$

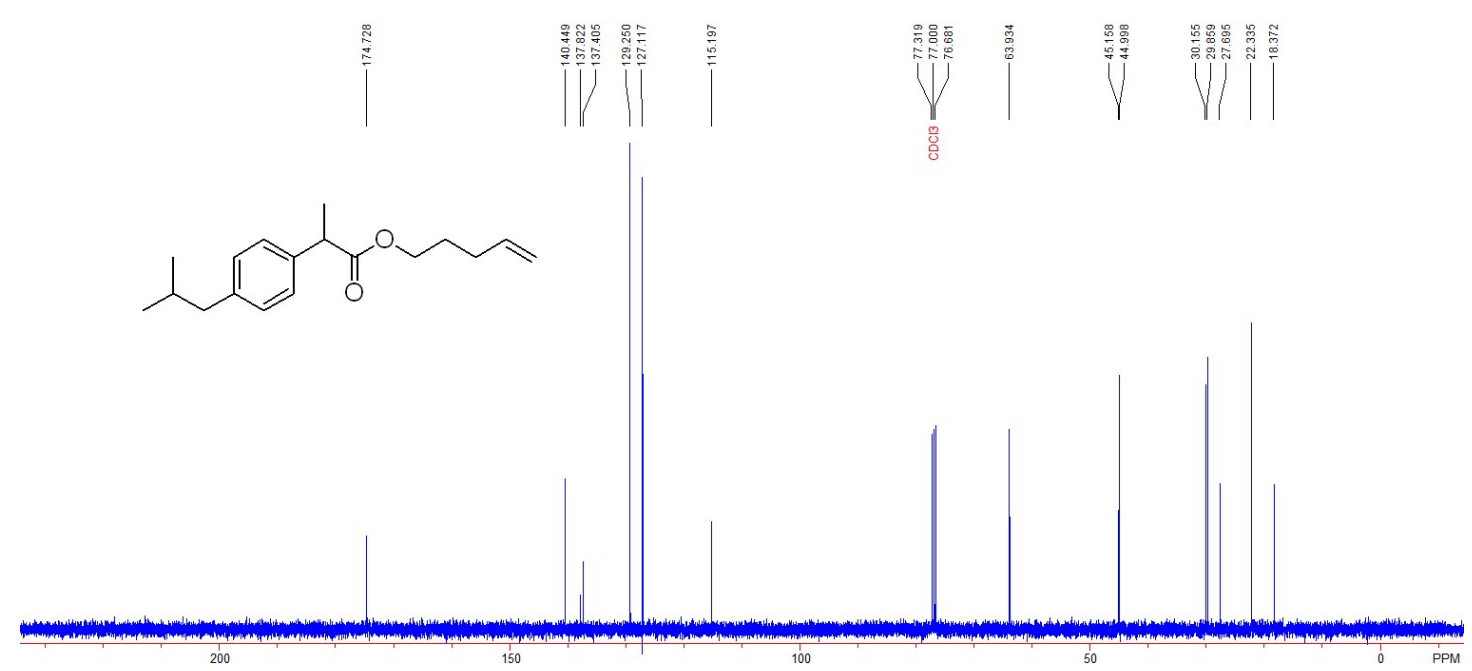




\section{Compound 1ze}

${ }^{1} \mathrm{H}$ NMR (400 MHz, $\mathrm{CDCl}_{3}$ )
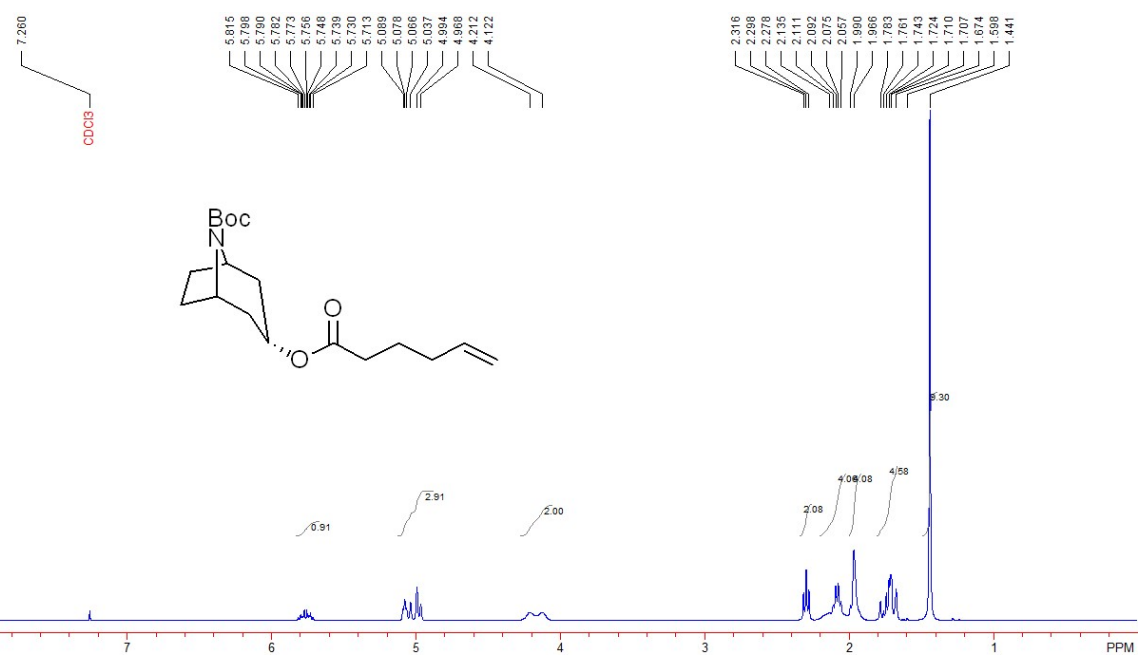

\section{Compound 1ze}

${ }^{13} \mathrm{C}$ NMR (101 MHz, $\left.\mathrm{CDCl}_{3}\right)$
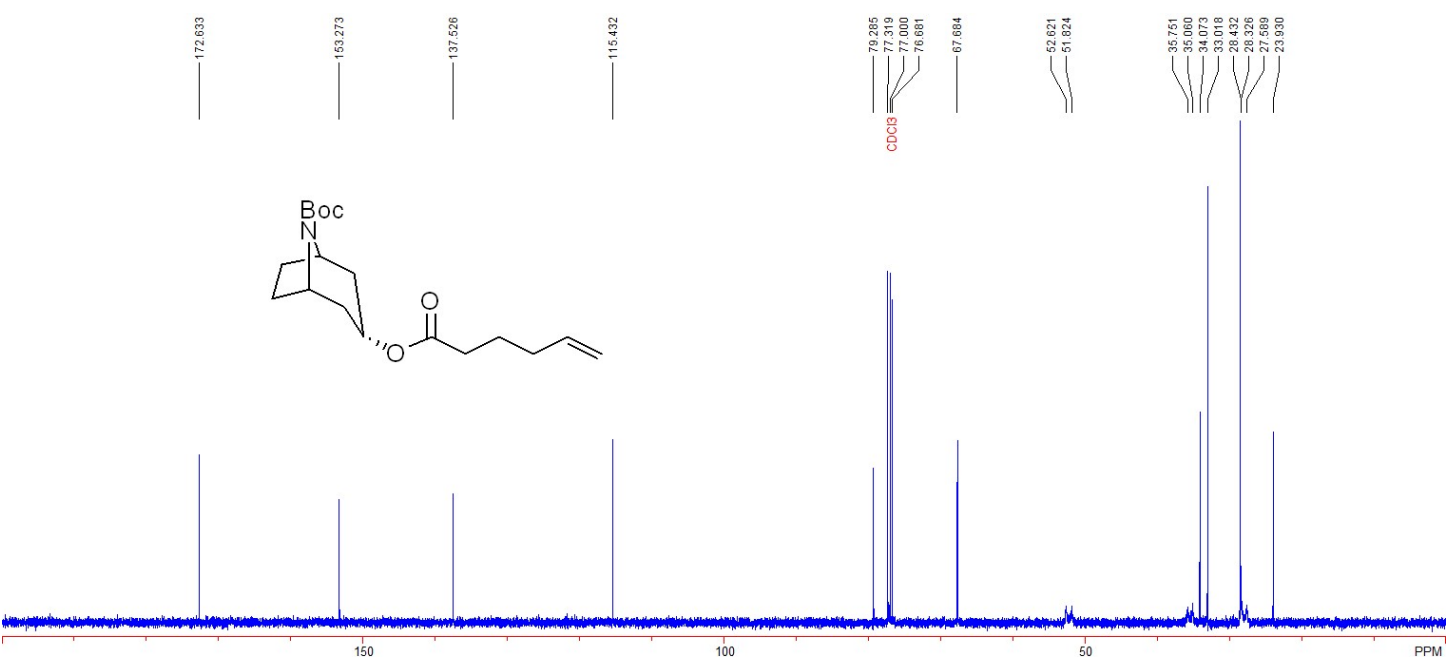
Compound 3a ${ }^{1} \mathrm{H}$ NMR (400 MHz, $\left.\mathrm{CDCl}_{3}\right)$

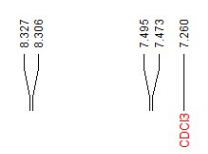
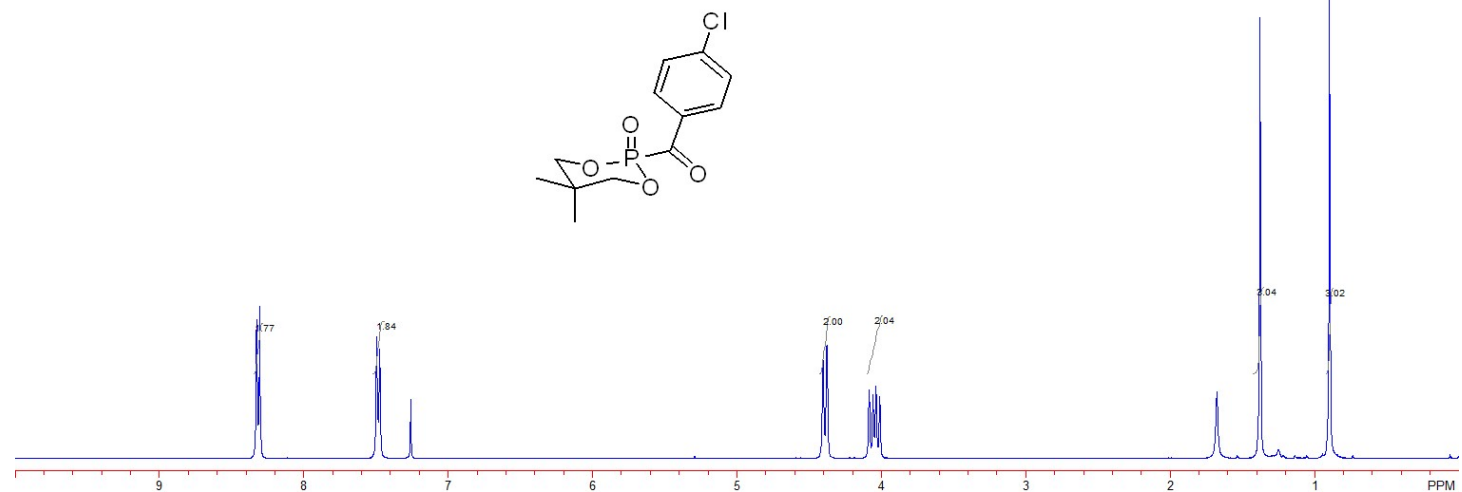

Compound 3a ${ }^{31} \mathrm{P}$ NMR (163 MHz, $\left.\mathrm{CDCl}_{3}\right)$

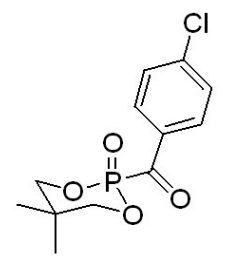

Compound 3a ${ }^{13} \mathrm{C}$ NMR (101 MHz, $\left.\mathrm{CDCl}_{3}\right)$

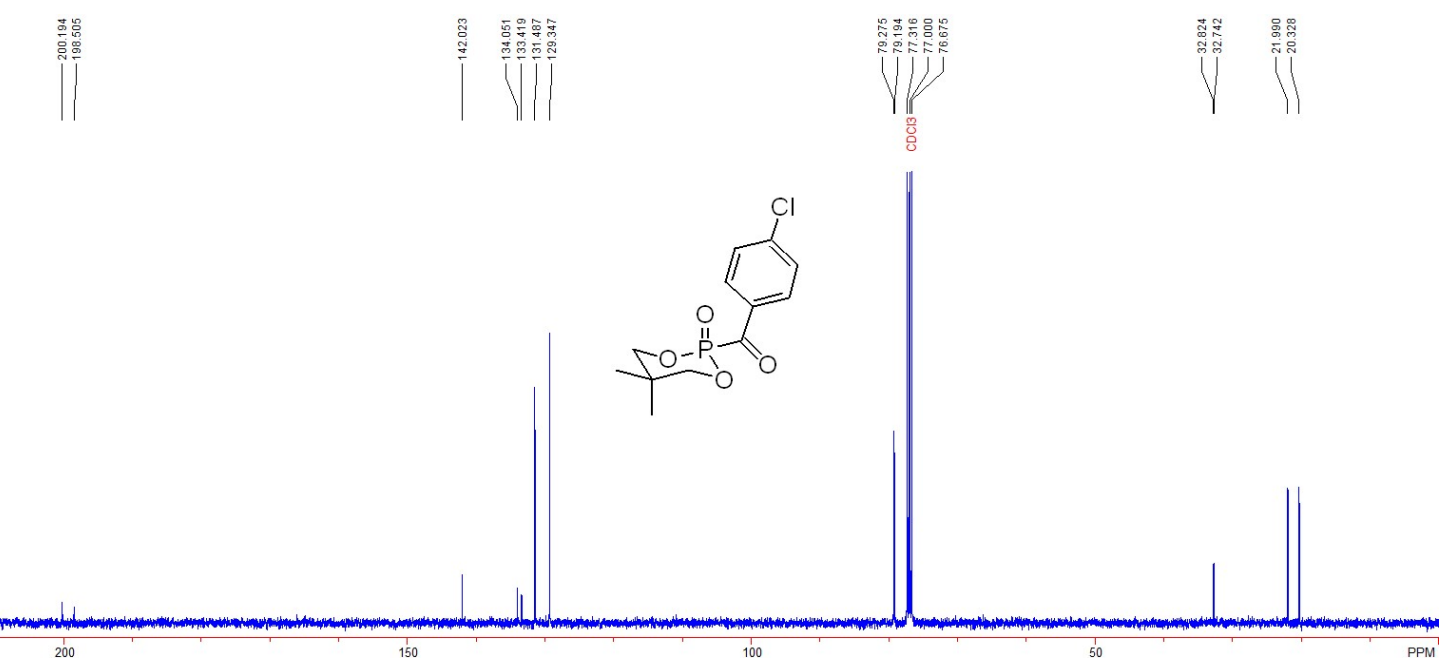


Compound 3b ${ }^{1} \mathrm{H}$ NMR (400 MHz, $\left.\mathrm{CDCl}_{3}\right)$

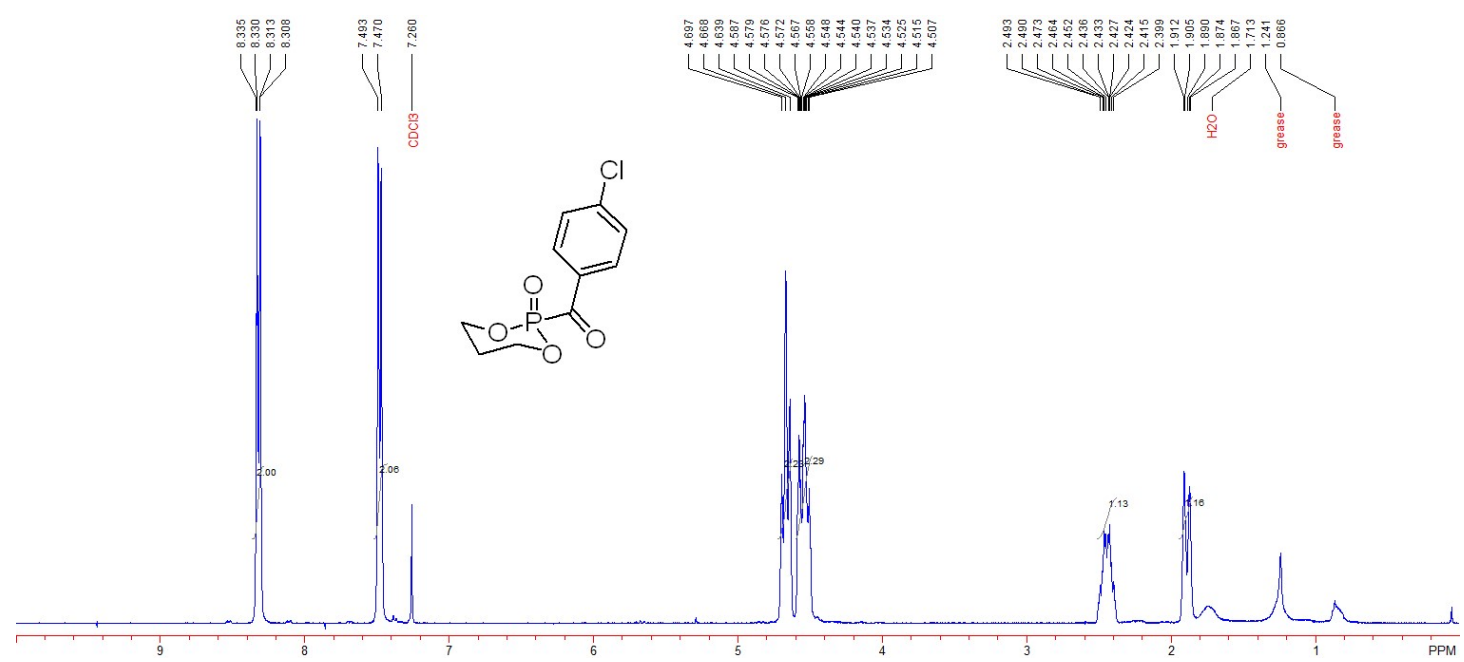

Compound 3b ${ }^{31} \mathrm{P}$ NMR (163 MHz, $\mathrm{CDCl}_{3}$ )

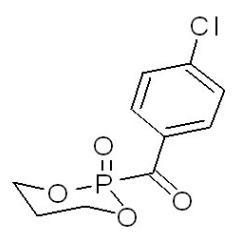

Compound 3b ${ }^{13} \mathrm{C}$ NMR (101 MHz, $\left.\mathrm{CDCl}_{3}\right)$
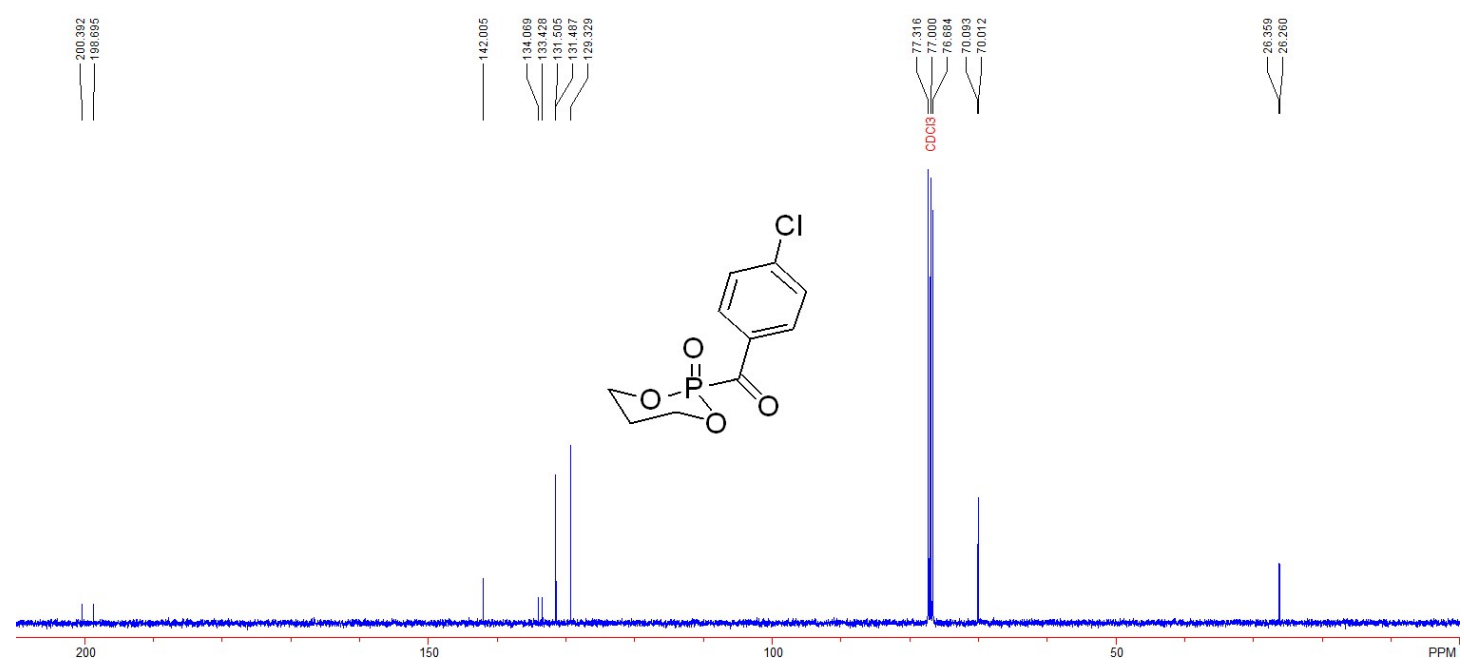
Compound 3e ${ }^{1} \mathrm{H}$ NMR (400 MHz, $\left.\mathrm{CDCl}_{3}\right)$

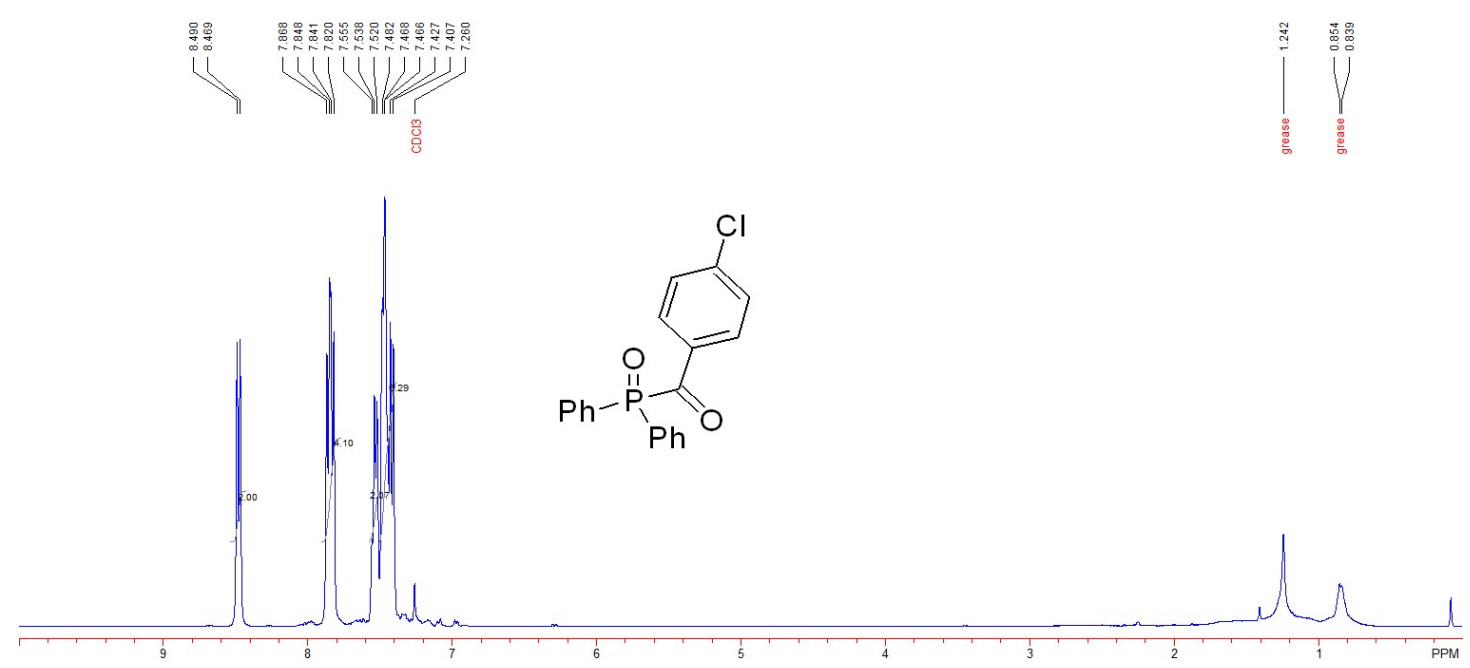

Compound 3e ${ }^{31} \mathrm{P}$ NMR (163 MHz, $\left.\mathrm{CDCl}_{3}\right)$

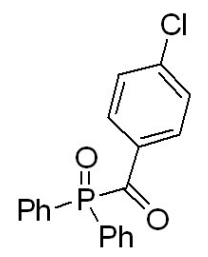

Compound 3e ${ }^{13} \mathrm{C}$ NMR (101 $\left.\mathrm{MHz}, \mathrm{CDCl}_{3}\right)$

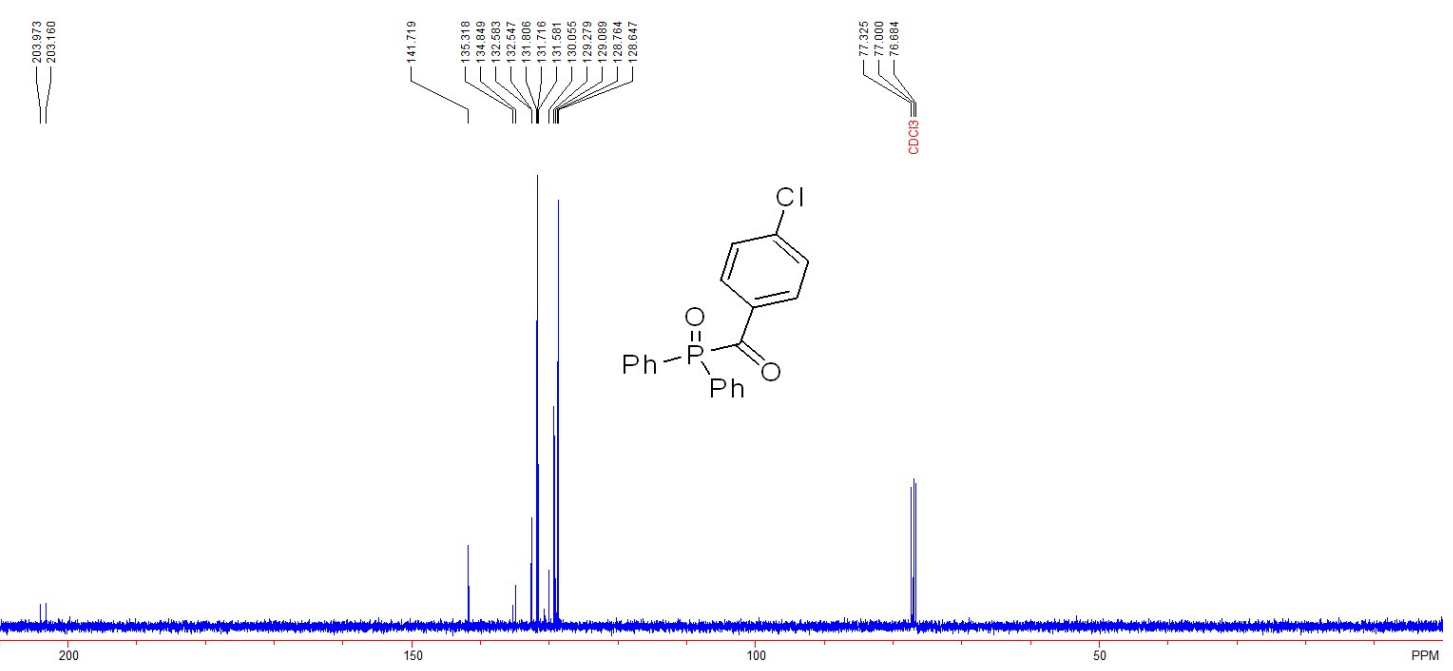


Compound $3 f{ }^{1} \mathrm{H}$ NMR $\left(400 \mathrm{MHz}, \mathrm{CDCl}_{3}\right)$
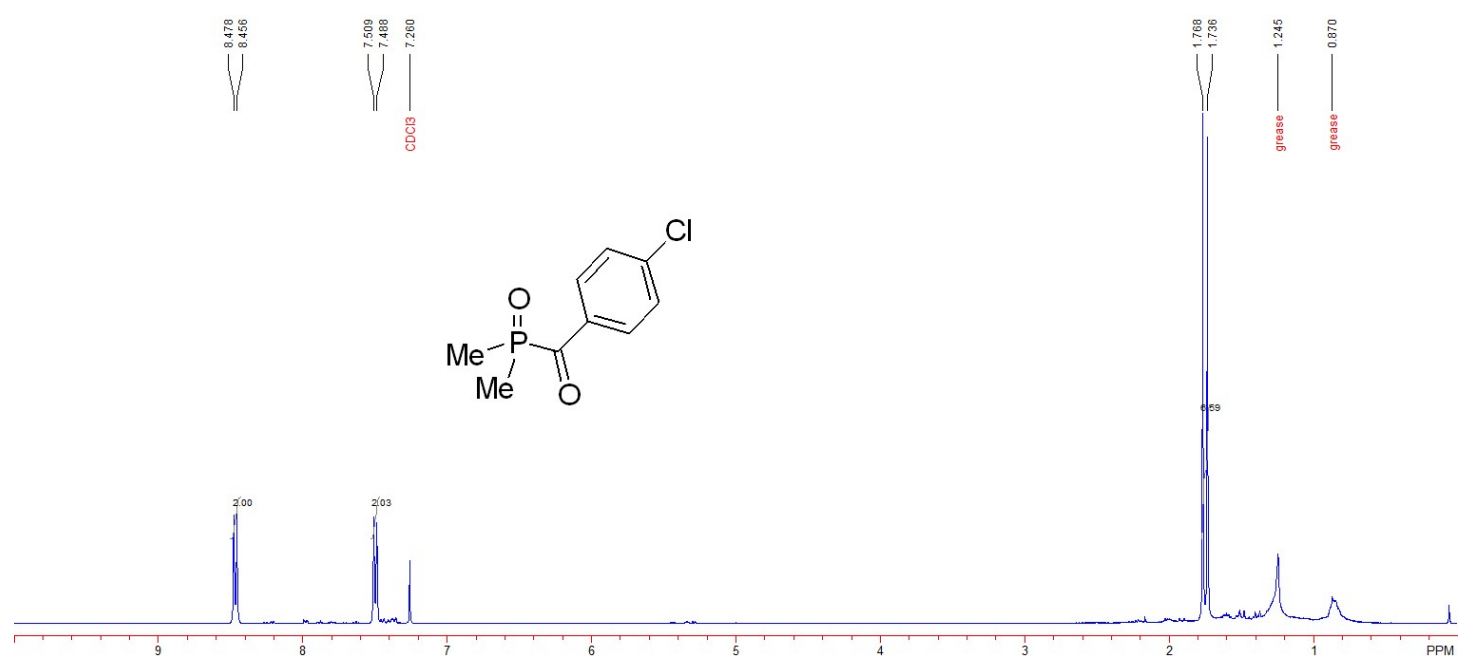

Compound 3f ${ }^{31} \mathrm{P}$ NMR (163 MHz, $\mathrm{CDCl}_{3}$ )<smiles>CP(C)(=O)C(=O)c1ccc(Cl)cc1</smiles>

Compound 3f ${ }^{13} \mathrm{C}$ NMR (125 MHz, $\left.\mathrm{CDCl}_{3}\right)$

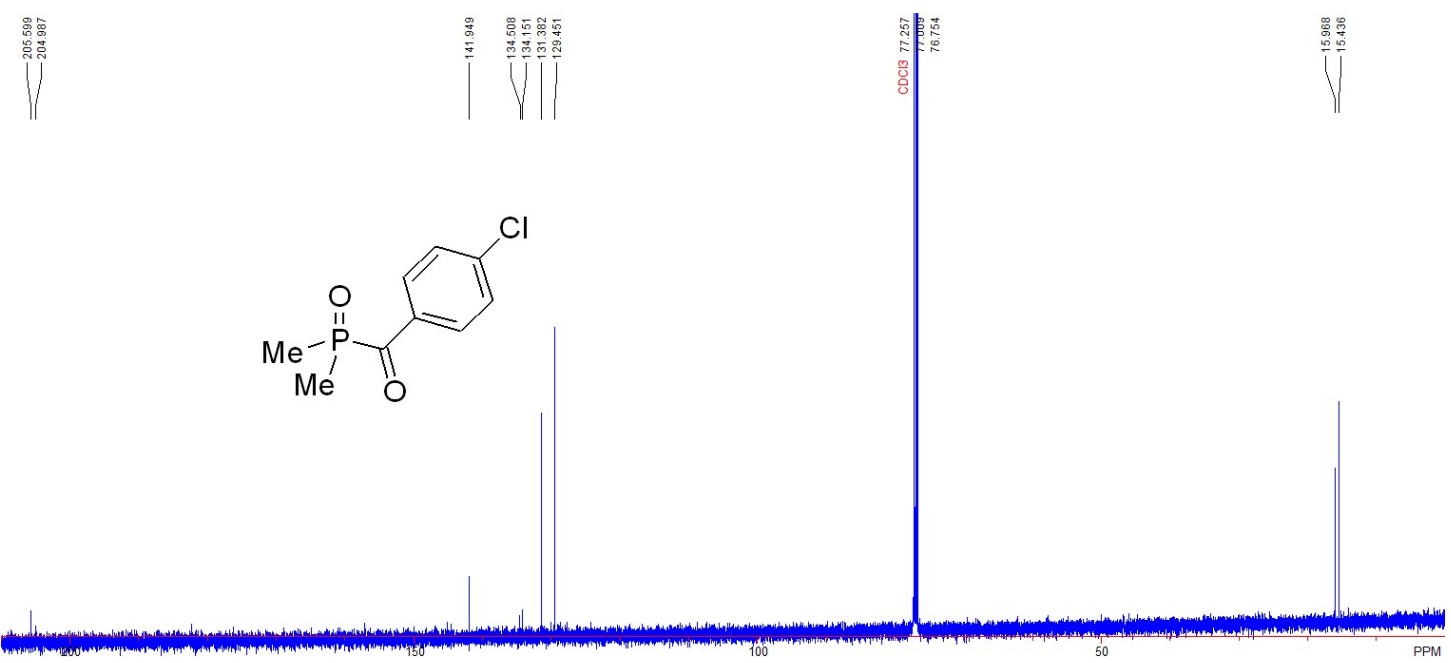


Compound 3g ${ }^{1} \mathrm{H}$ NMR (400 MHz, $\left.\mathrm{CDCl}_{3}\right)$

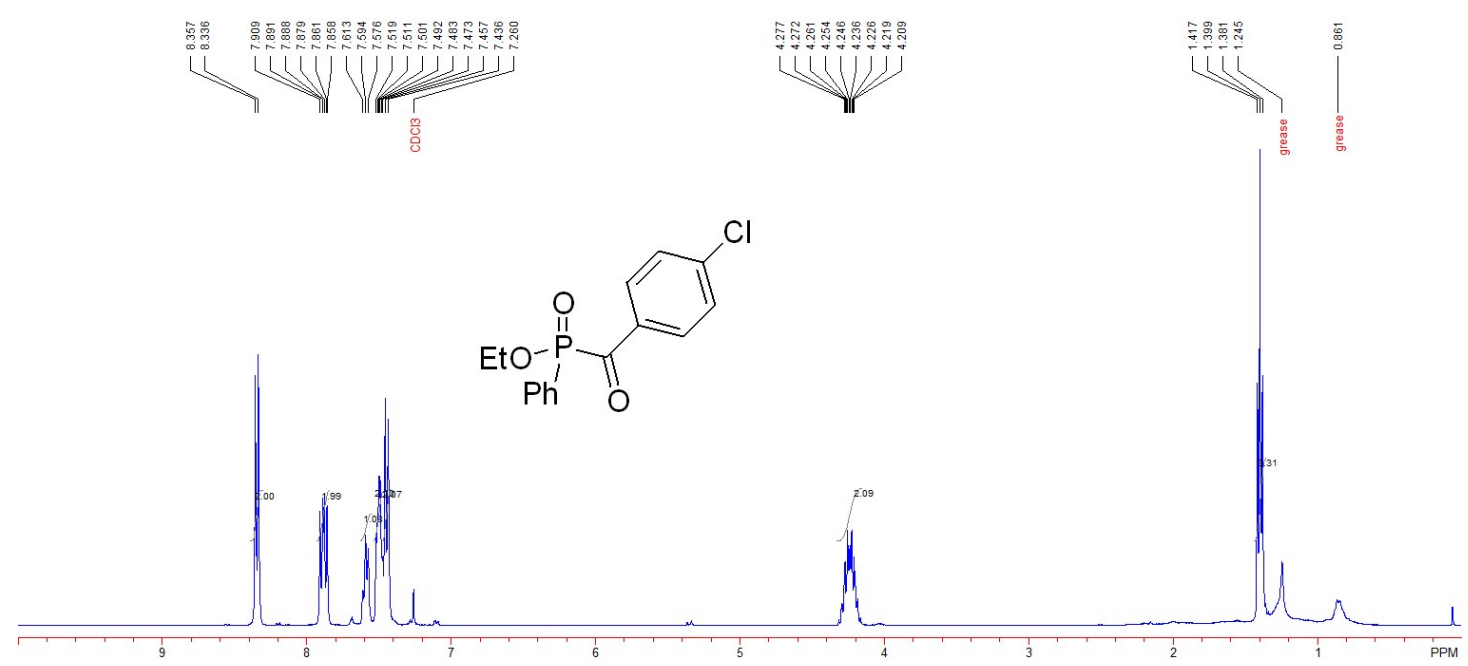

Compound 3g ${ }^{31} \mathrm{P}$ NMR (163 MHz, $\left.\mathrm{CDCl}_{3}\right)$

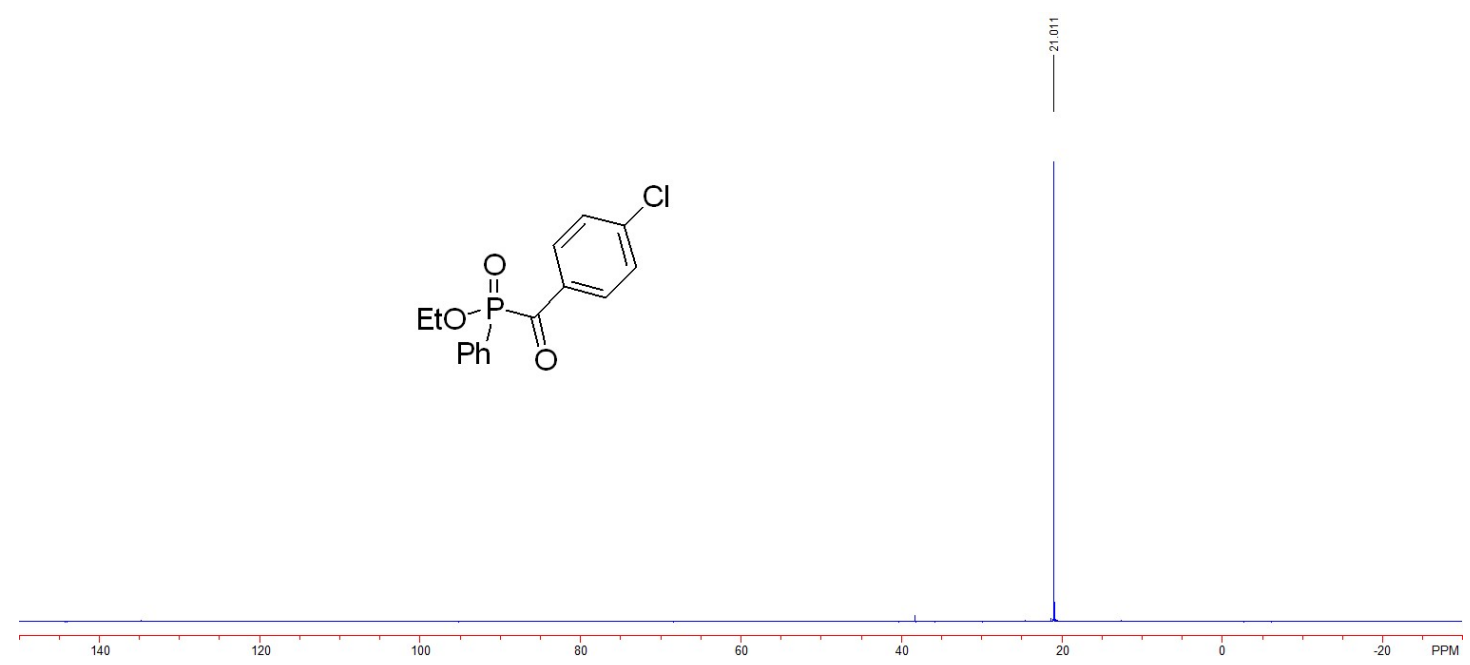

Compound 3g ${ }^{13} \mathrm{C}$ NMR (125 MHz, $\left.\mathrm{CDCl}_{3}\right)$

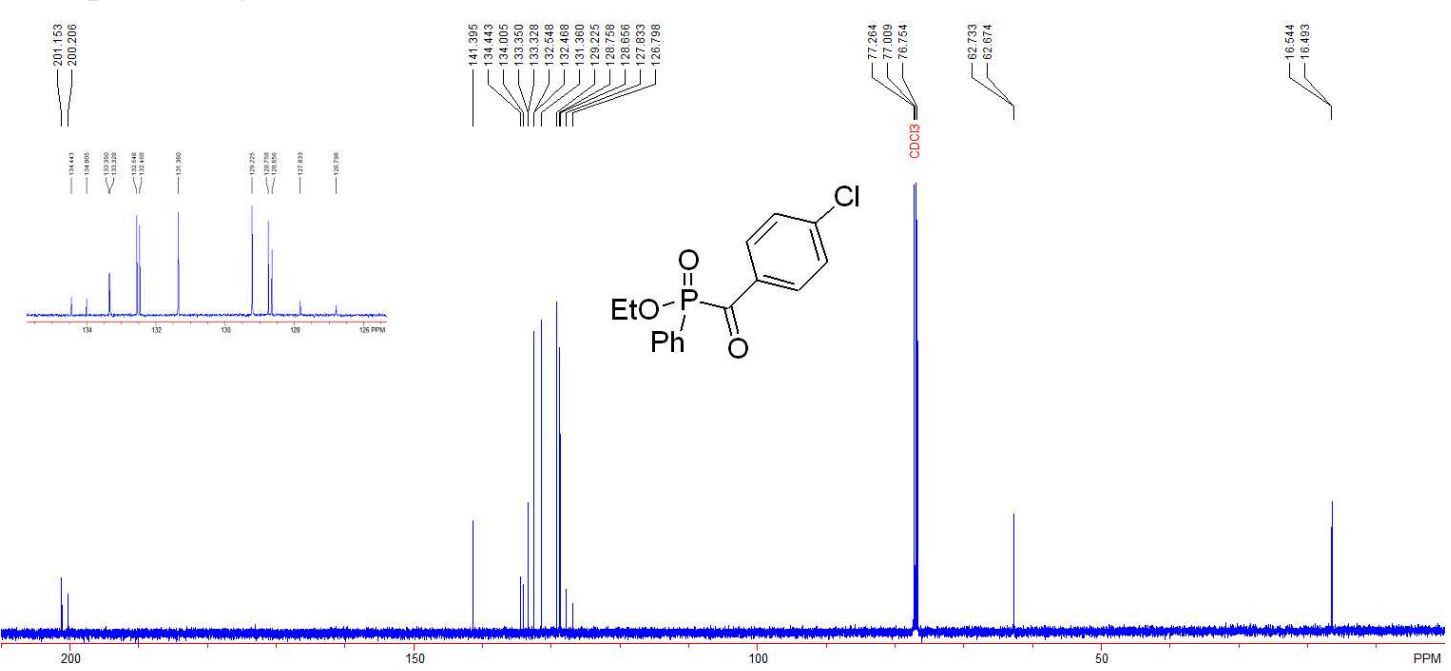


Compound 3h ${ }^{1} \mathrm{H}$ NMR (400 MHz, $\left.\mathrm{CDCl}_{3}\right)$

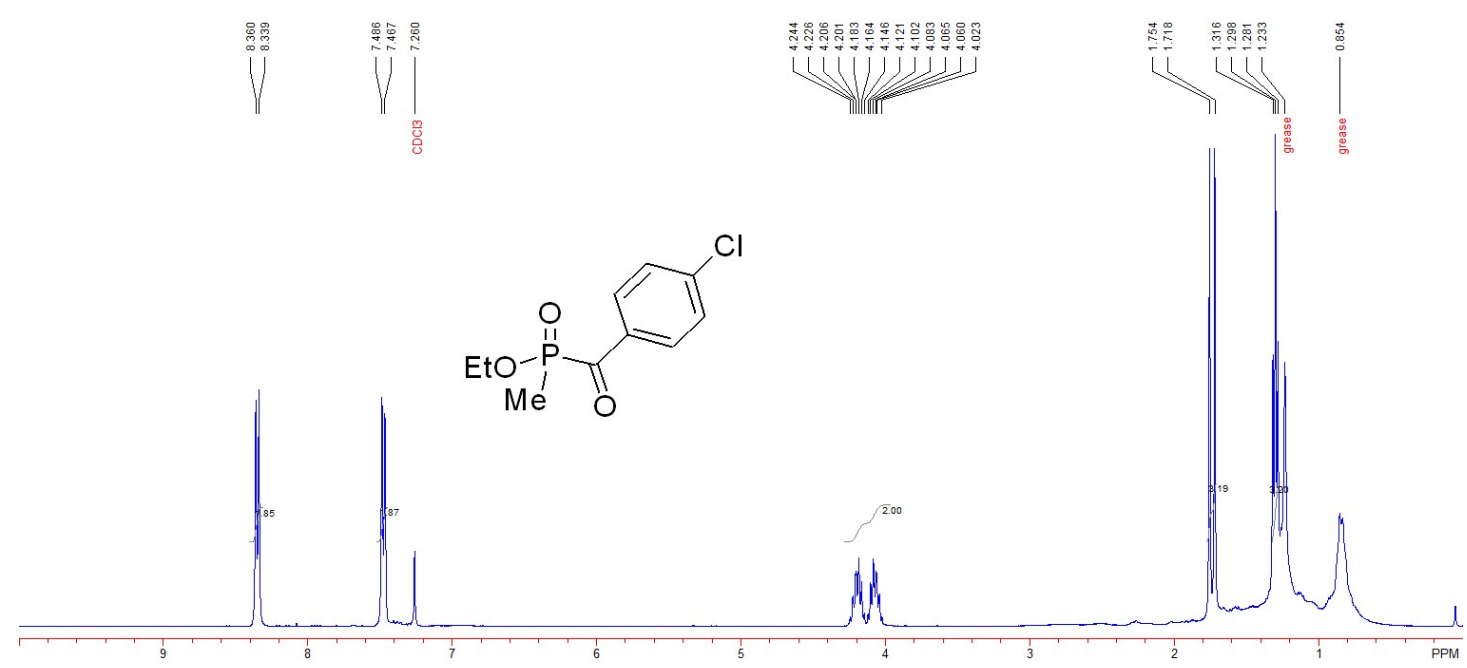

Compound 3h ${ }^{31} \mathrm{P}$ NMR (163 MHz, $\mathrm{CDCl}_{3}$ )
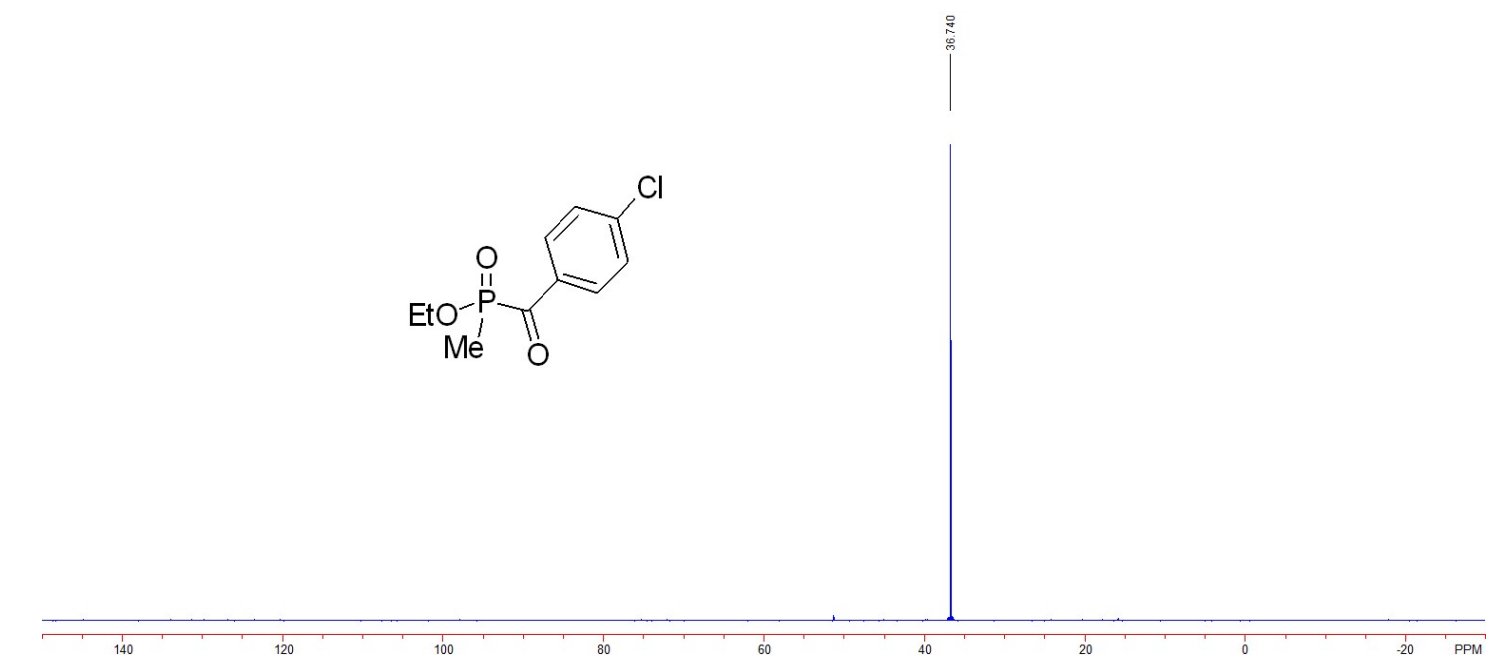

Compound $3 \mathbf{h}{ }^{13} \mathrm{C}$ NMR (125 MHz, $\left.\mathrm{CDCl}_{3}\right)$

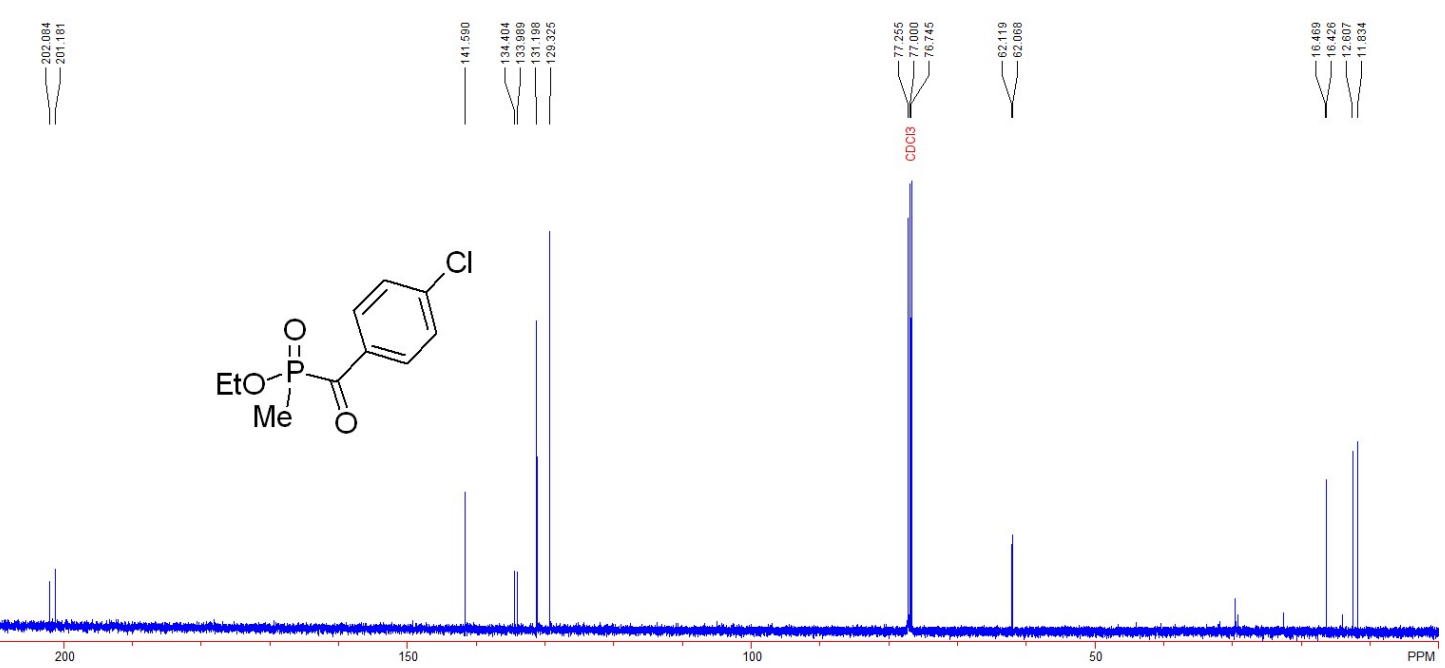


Compound 5a ${ }^{1} \mathrm{H}$ NMR (400 MHz, $\left.\mathrm{CDCl}_{3}\right)$
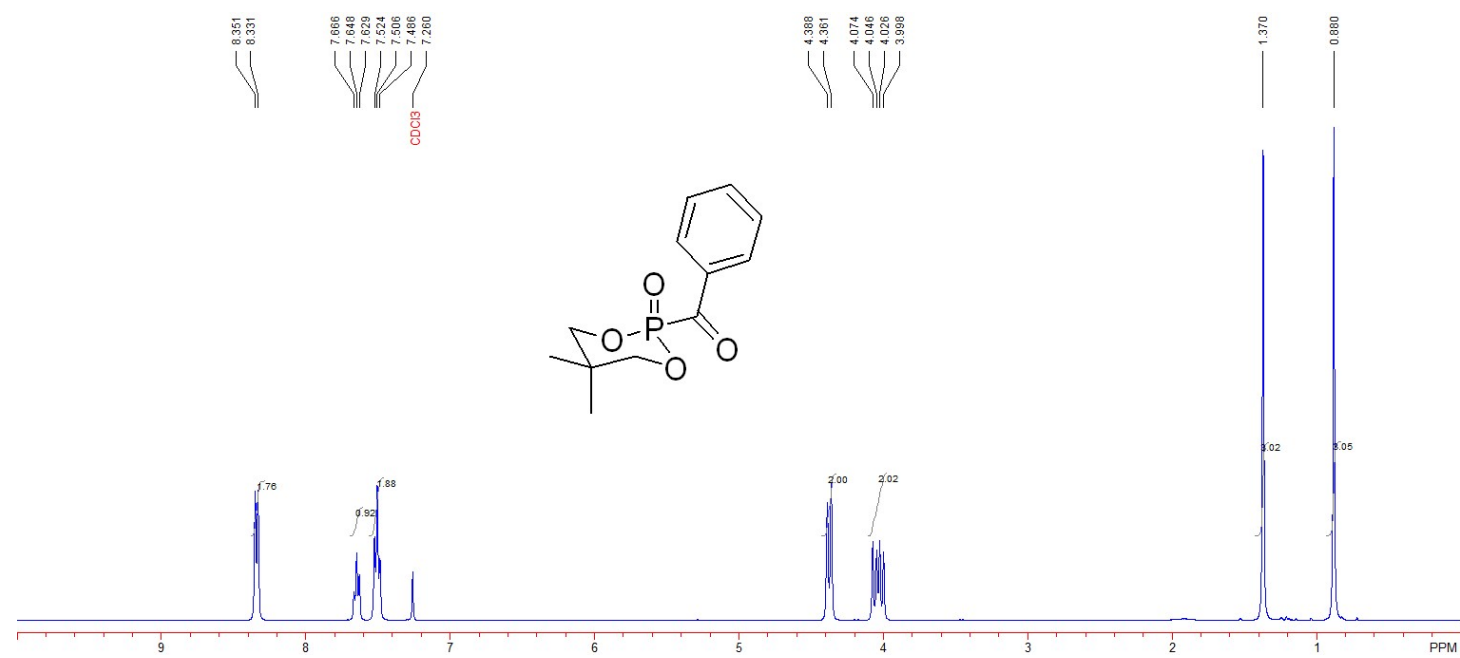

Compound 5a ${ }^{31} \mathrm{P}$ NMR (163 MHz, $\left.\mathrm{CDCl}_{3}\right)$

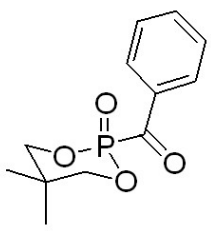

Compound 5a ${ }^{13} \mathrm{C}$ NMR (101 MHz, $\left.\mathrm{CDCl}_{3}\right)$
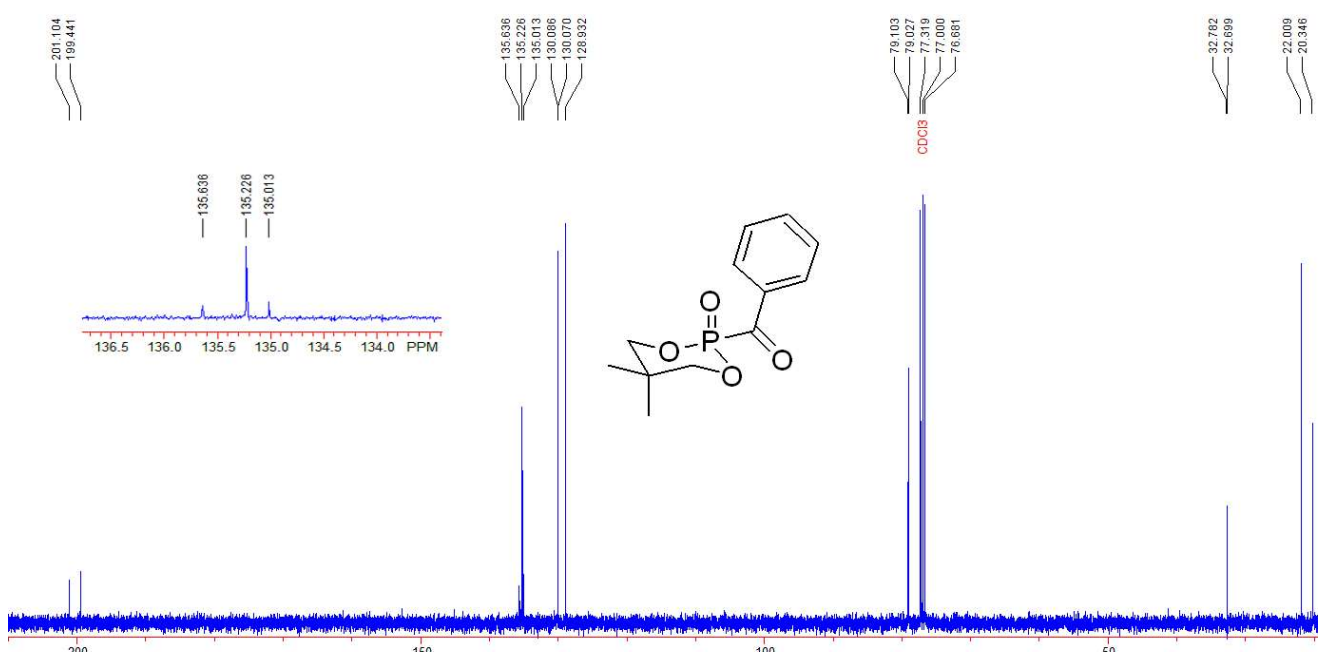


\section{Compound 5b}

${ }^{1} \mathrm{H}$ NMR (400 MHz, $\mathrm{CDCl}_{3}$ )

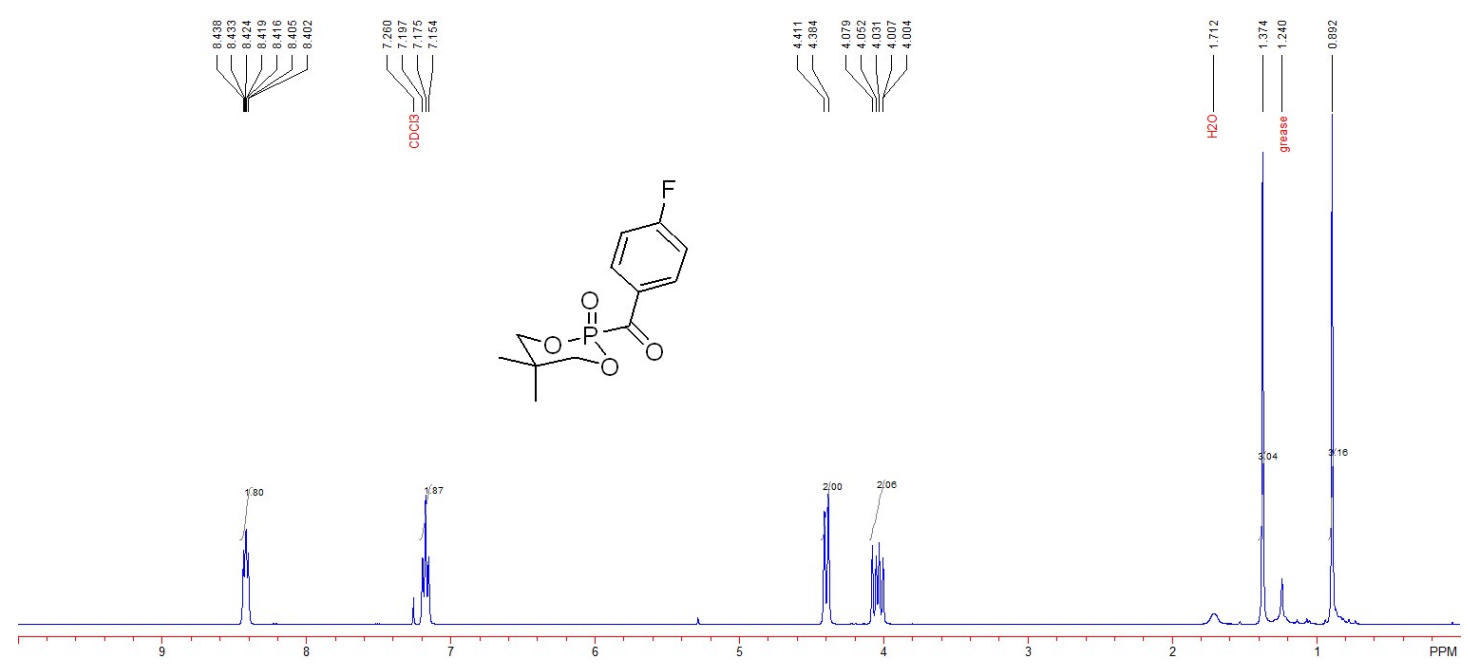

\section{Compound 5b}

${ }^{31} \mathrm{P}$ NMR (163 MHz, $\left.\mathrm{CDCl}_{3}\right)$

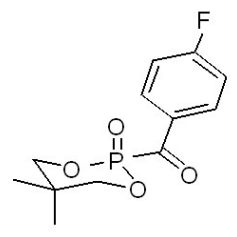




\section{Compound 5b}

${ }^{19} \mathrm{~F}$ NMR $\left(376 \mathrm{MHz}, \mathrm{CDCl}_{3}\right)$
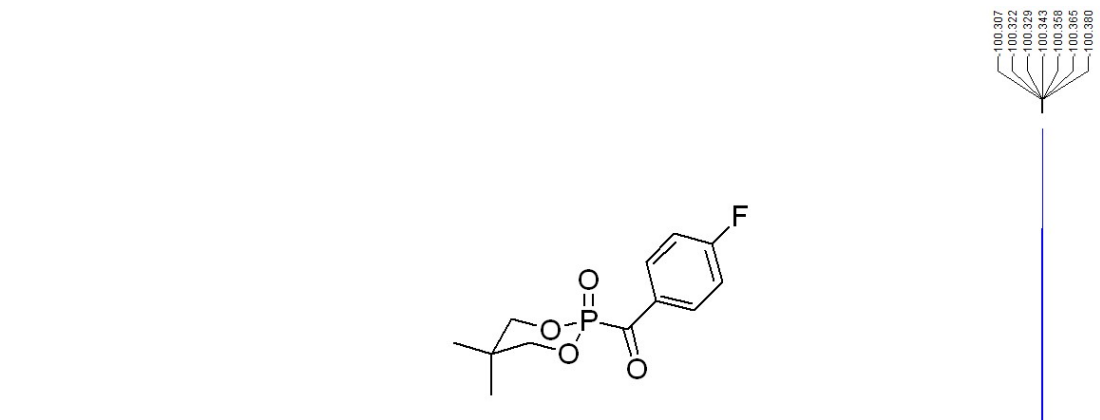

\section{Compound 5b}

${ }^{13} \mathrm{C} \mathrm{NMR}\left(101 \mathrm{MHz}, \mathrm{CDCl}_{3}\right)$
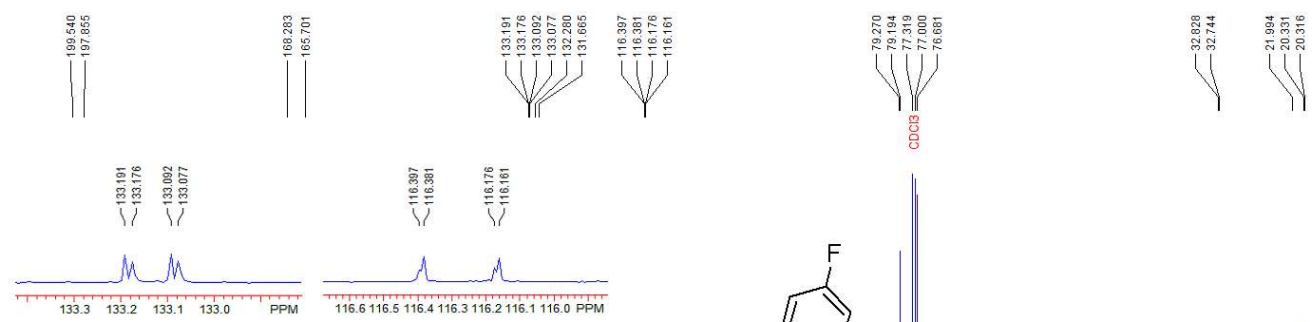
Compound 5c ${ }^{1} \mathrm{H}$ NMR (400 MHz, $\left.\mathrm{CDCl}_{3}\right)$<smiles>[B]C1=CC=[C]C1</smiles>
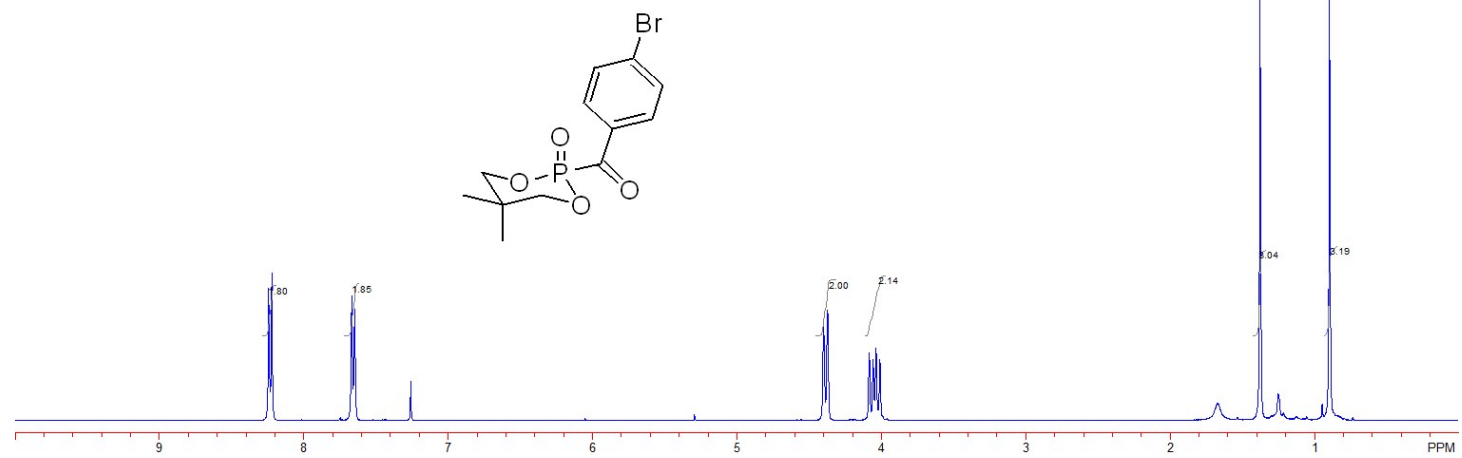

Compound 5c ${ }^{31} \mathrm{P}$ NMR (163 MHz, $\left.\mathrm{CDCl}_{3}\right)$

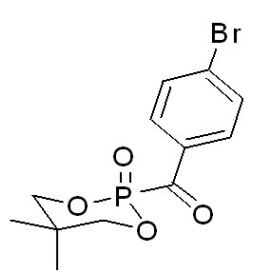

Compound 5c ${ }^{13} \mathrm{C}$ NMR (101 MHz, $\left.\mathrm{CDCl}_{3}\right)$

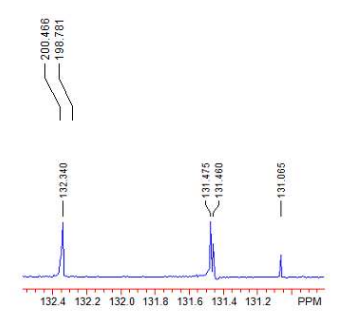

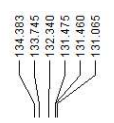
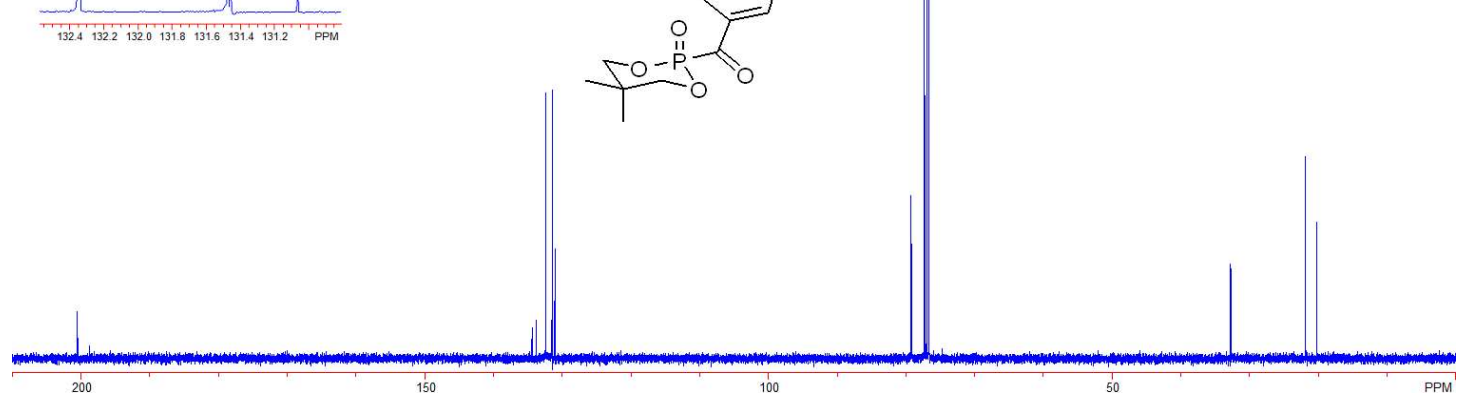
Compound 5d ${ }^{1} \mathrm{H}$ NMR (400 MHz, $\left.\mathrm{CDCl}_{3}\right)$<smiles>C=CC=[18O]</smiles>
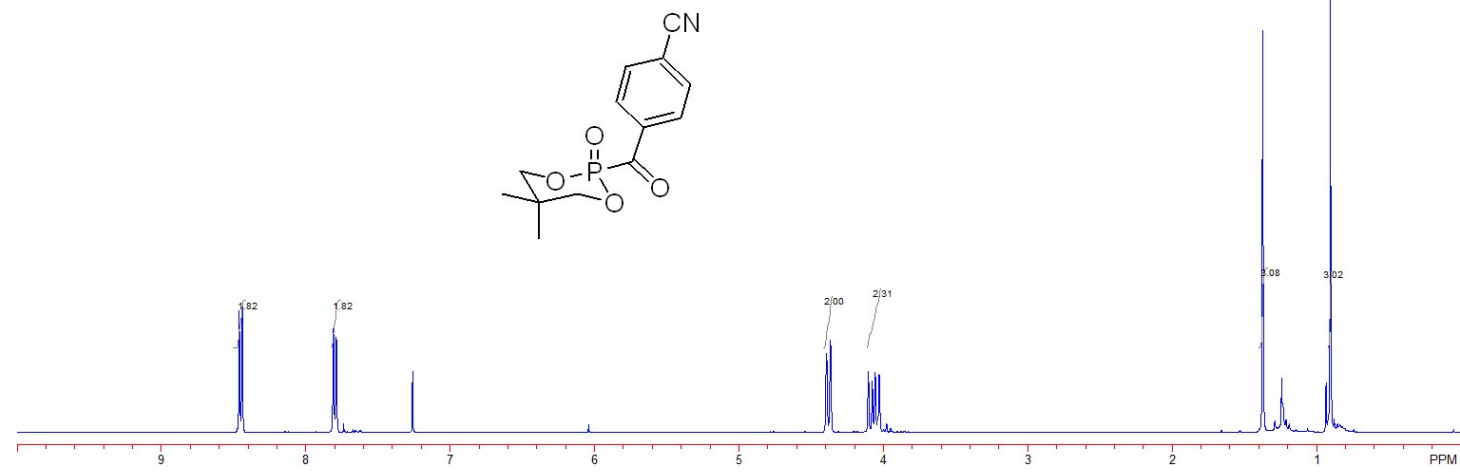

Compound 5d ${ }^{31} \mathrm{P}$ NMR (163 MHz, $\left.\mathrm{CDCl}_{3}\right)$

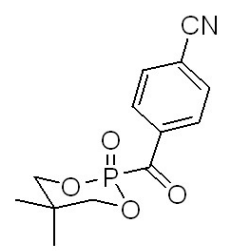

Compound 5d ${ }^{13} \mathrm{C}$ NMR (101 MHz, $\left.\mathrm{CDCl}_{3}\right)$
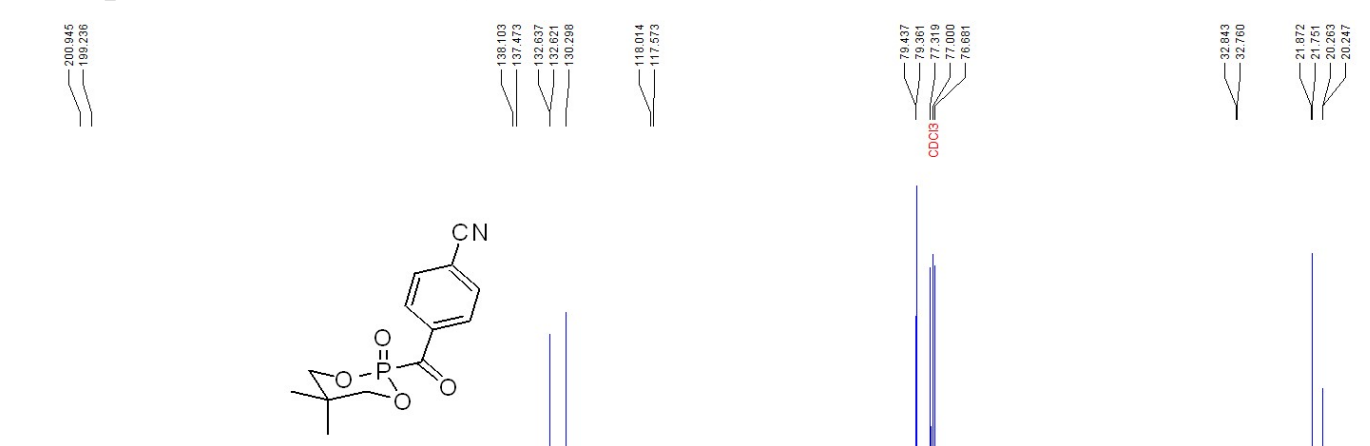
Compound 5e ${ }^{1} \mathrm{H}$ NMR (400 MHz, $\left.\mathrm{CDCl}_{3}\right)$

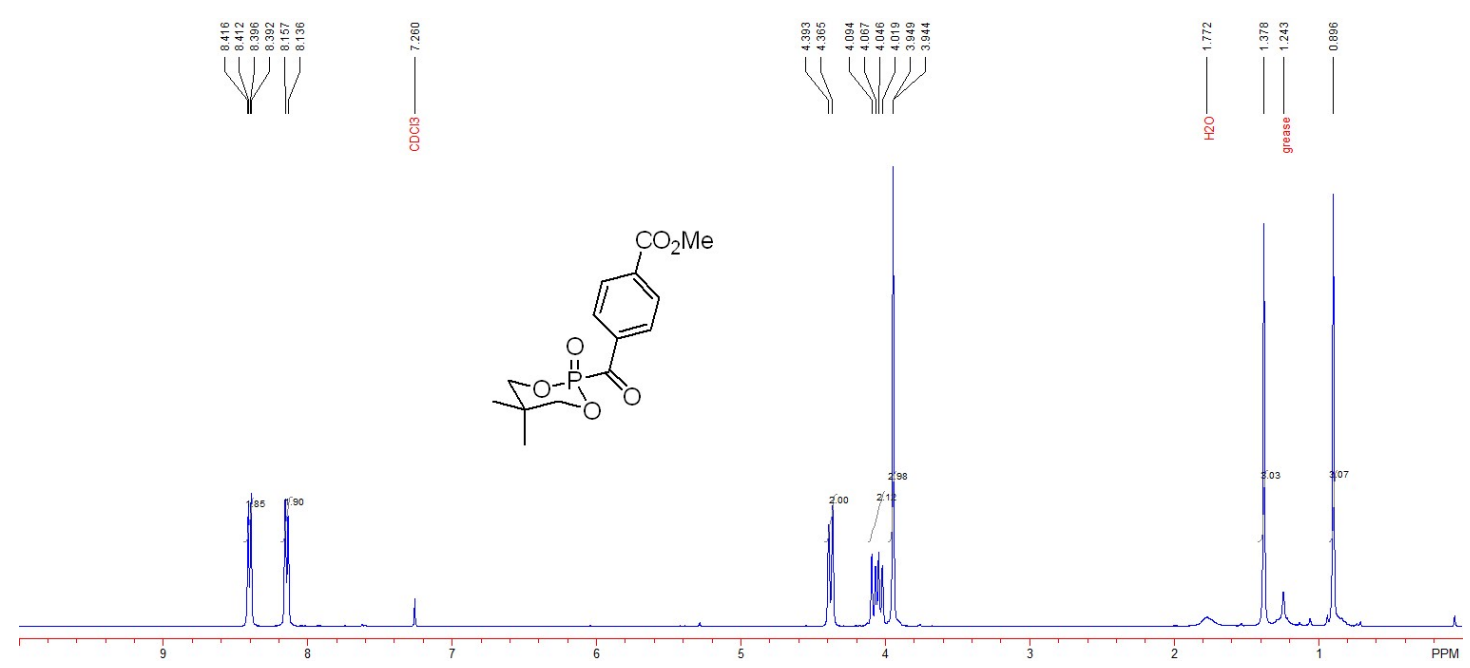

Compound 5e ${ }^{31} \mathrm{P}$ NMR (163 MHz, $\left.\mathrm{CDCl}_{3}\right)$

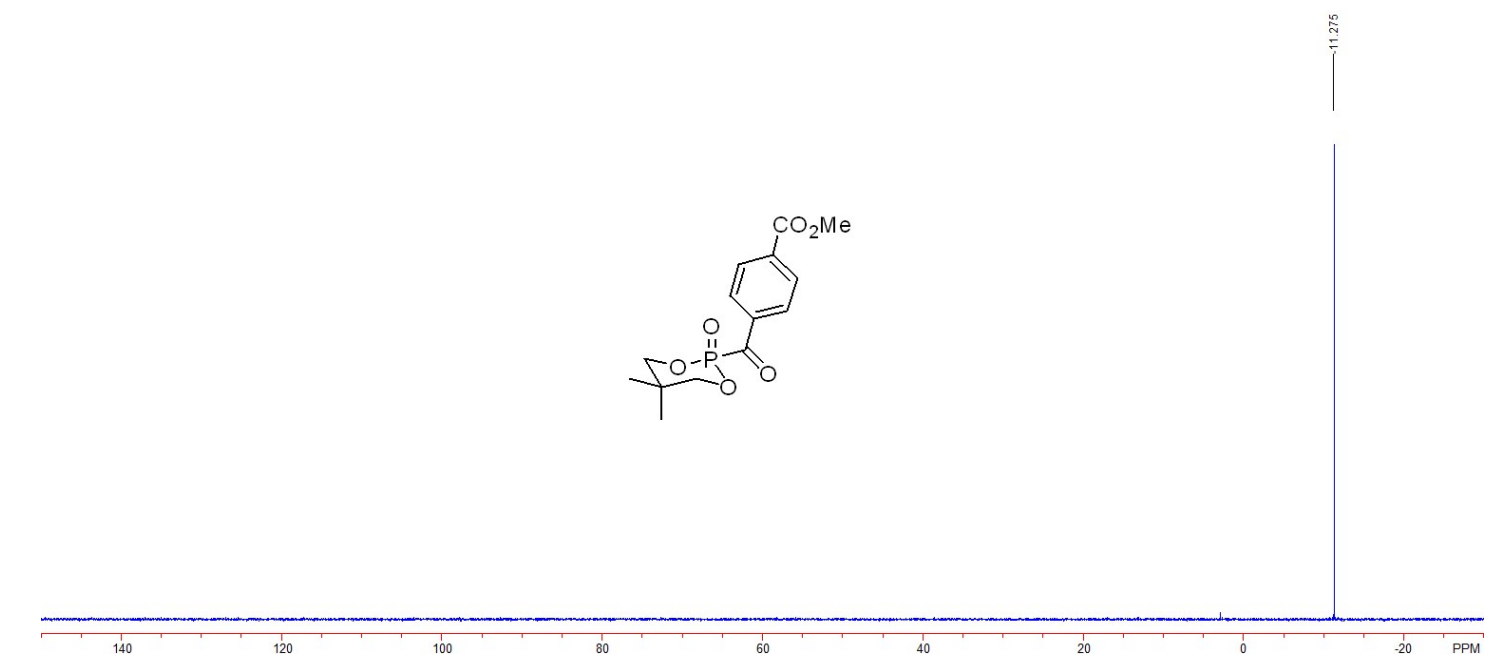

Compound 5e ${ }^{13} \mathrm{C}$ NMR (101 $\left.\mathrm{MHz}, \mathrm{CDCl}_{3}\right)$
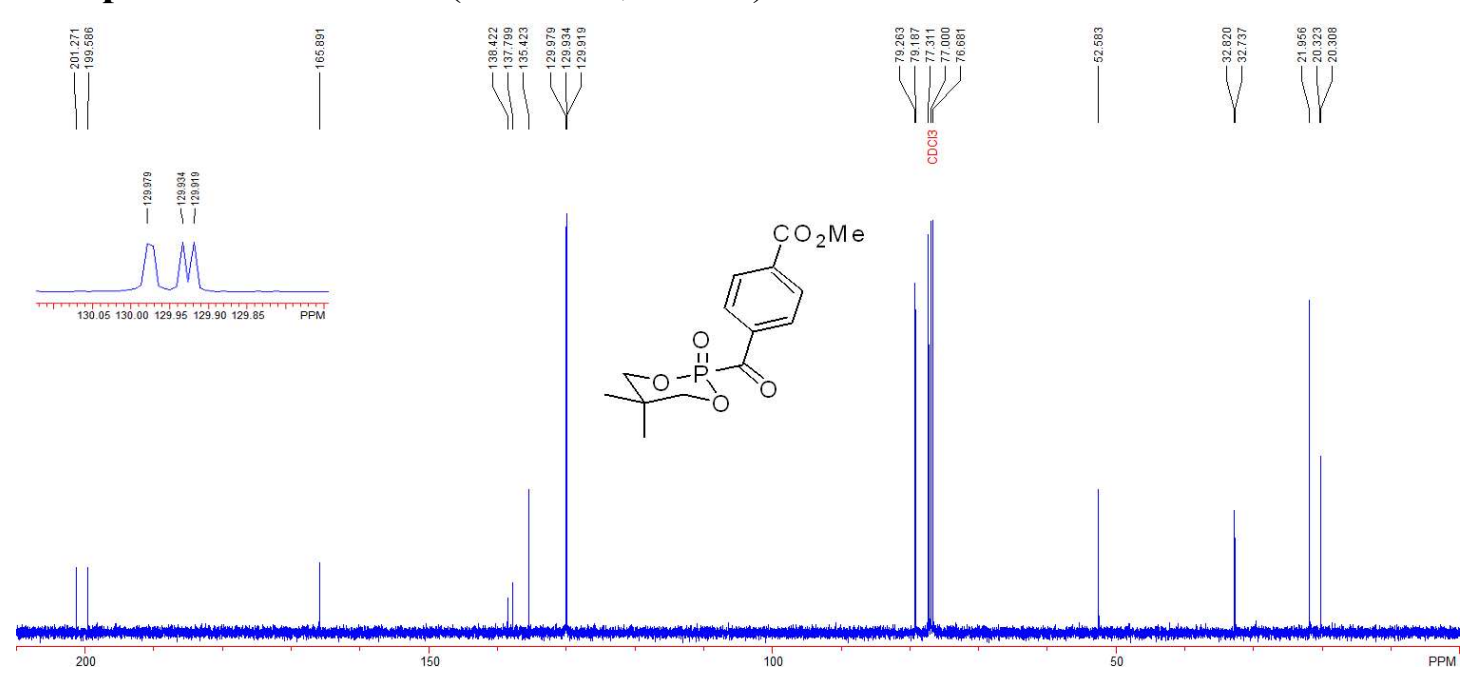


\section{Compound 5f}

${ }^{1} \mathrm{H}$ NMR (400 MHz, $\mathrm{CDCl}_{3}$ )
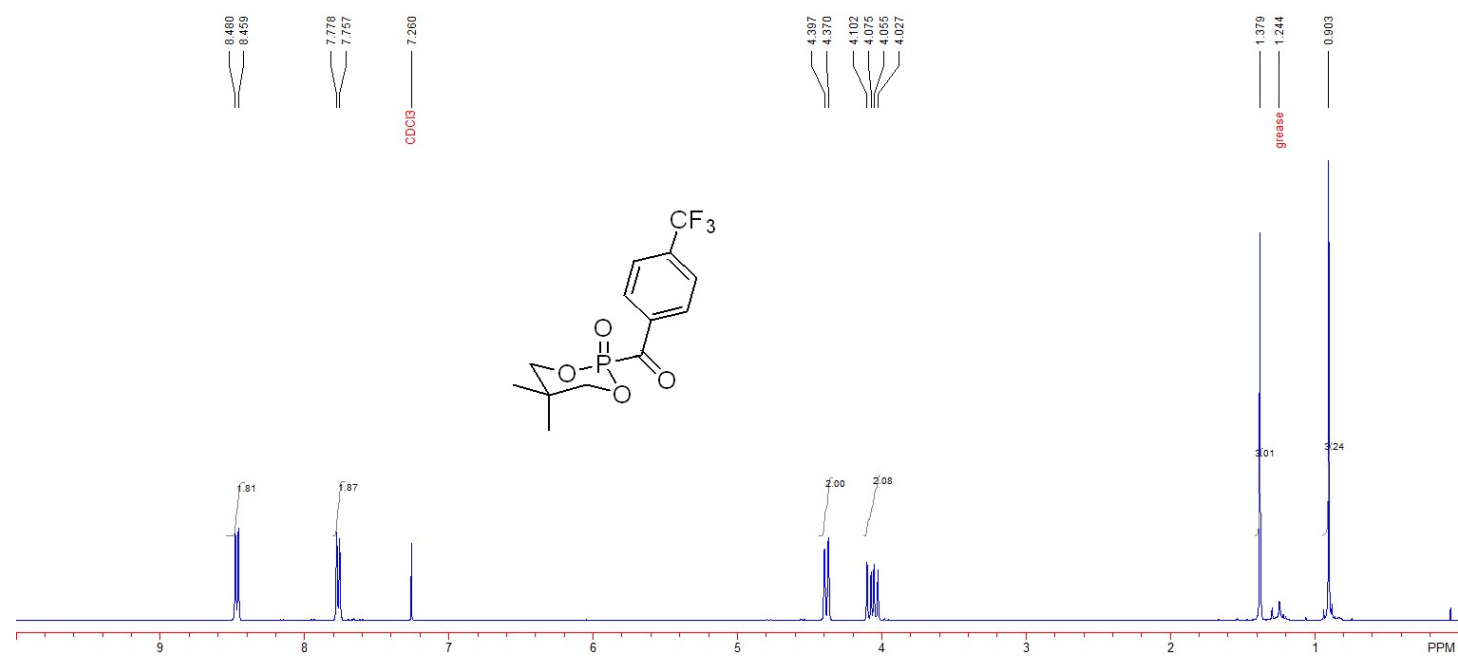

\section{Compound 5f}

${ }^{31} \mathrm{P}$ NMR (163 MHz, $\left.\mathrm{CDCl}_{3}\right)$

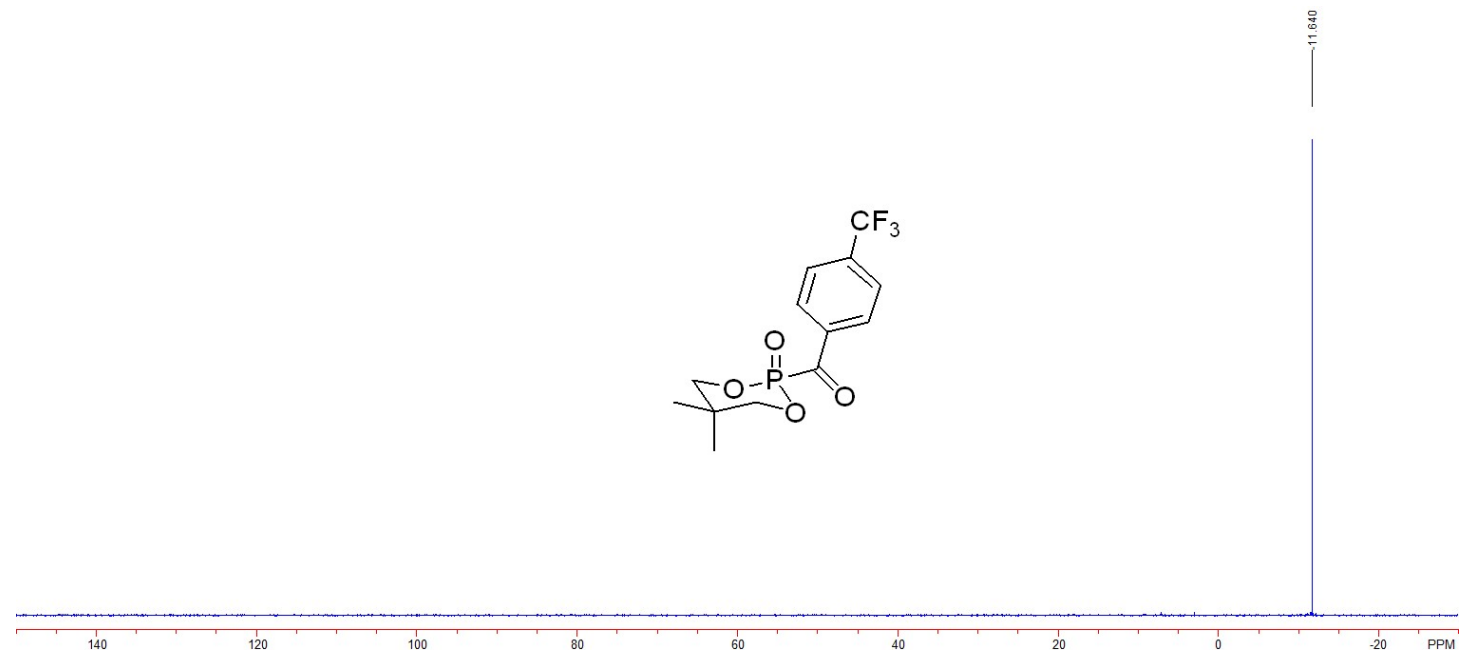




\section{Compound 5f}

${ }^{19} \mathrm{~F}$ NMR (376 MHz, $\mathrm{CDCl}_{3}$ )

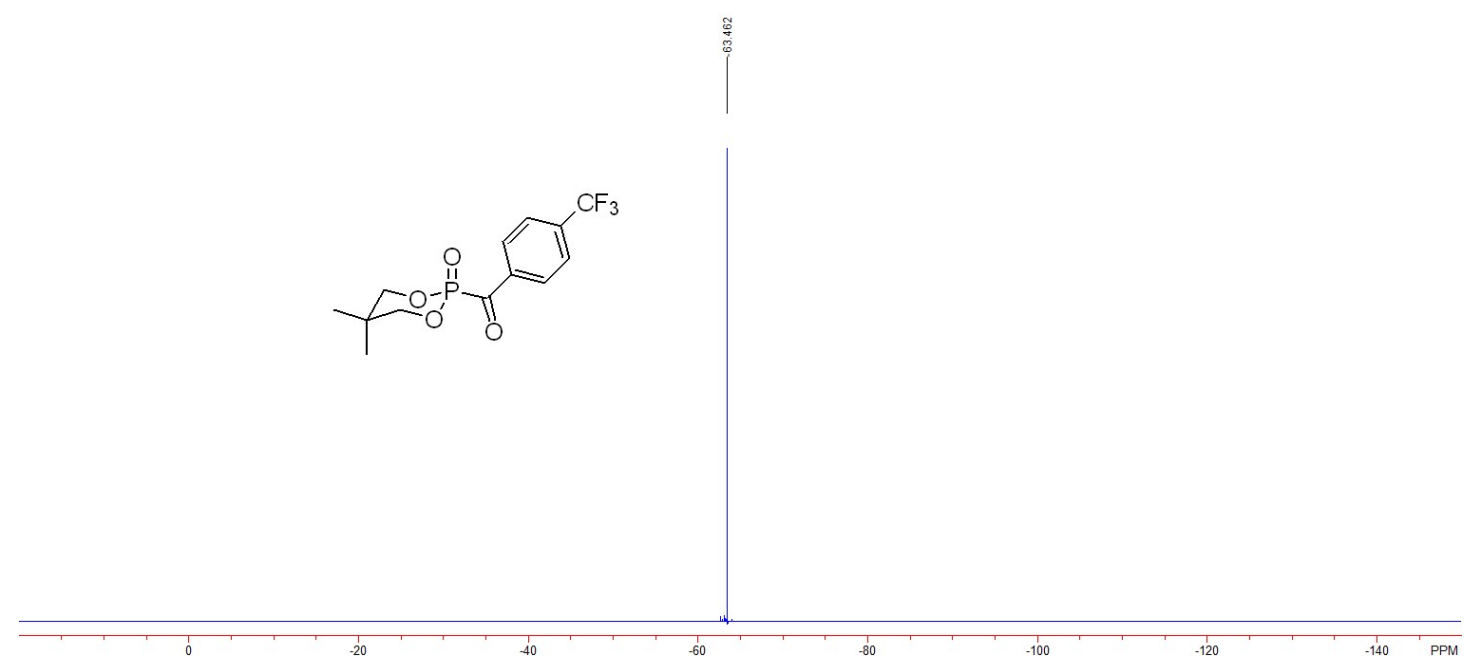

\section{Compound 5f}

${ }^{13} \mathrm{C} \mathrm{NMR}\left(101 \mathrm{MHz}, \mathrm{CDCl}_{3}\right)$
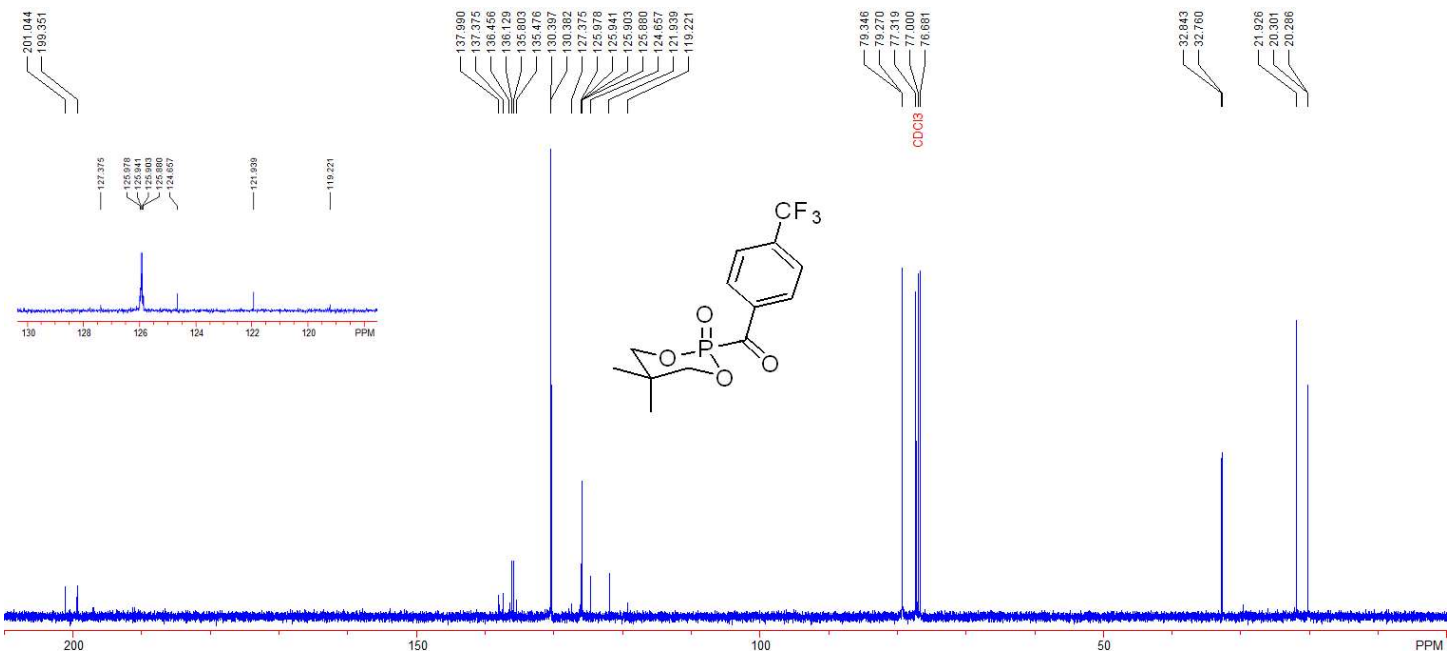


\section{Compound 5g}

${ }^{1} \mathrm{H}$ NMR (400 MHz, $\mathrm{CDCl}_{3}$ )
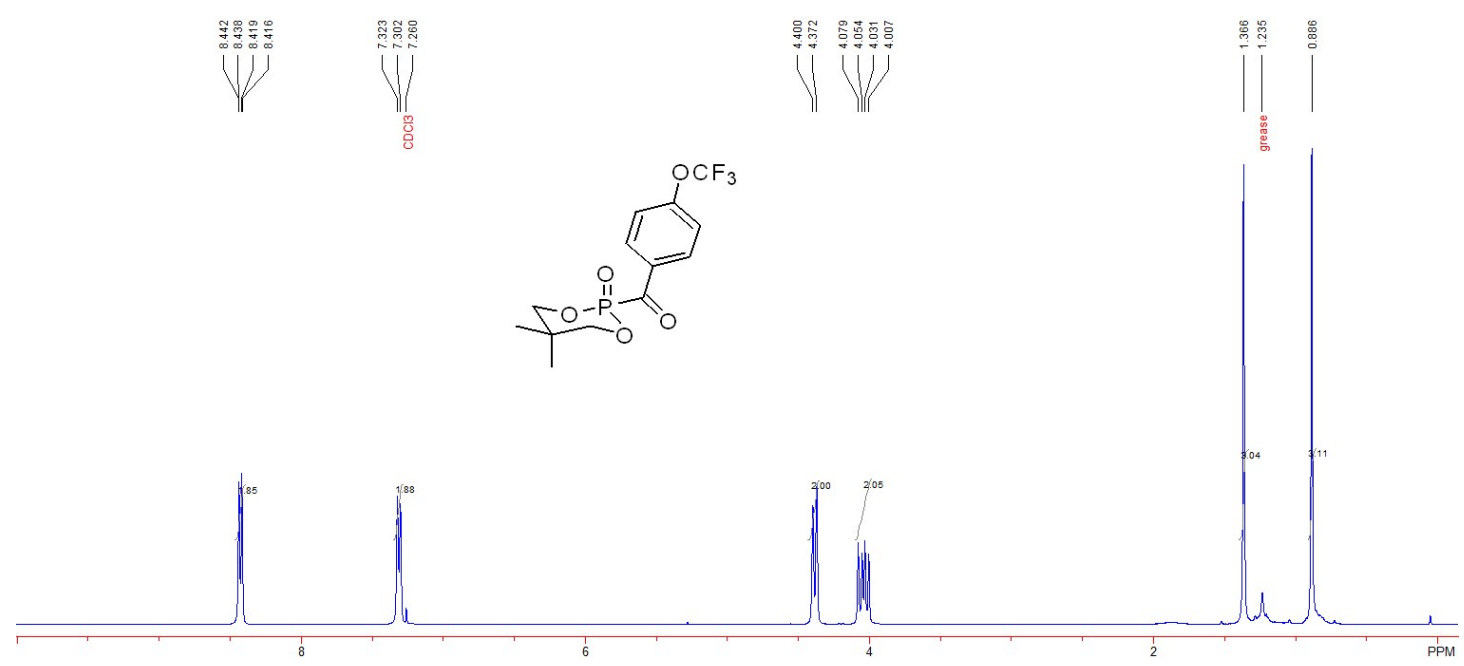

\section{Compound 5g}

${ }^{31} \mathrm{P}$ NMR (163 MHz, $\left.\mathrm{CDCl}_{3}\right)$

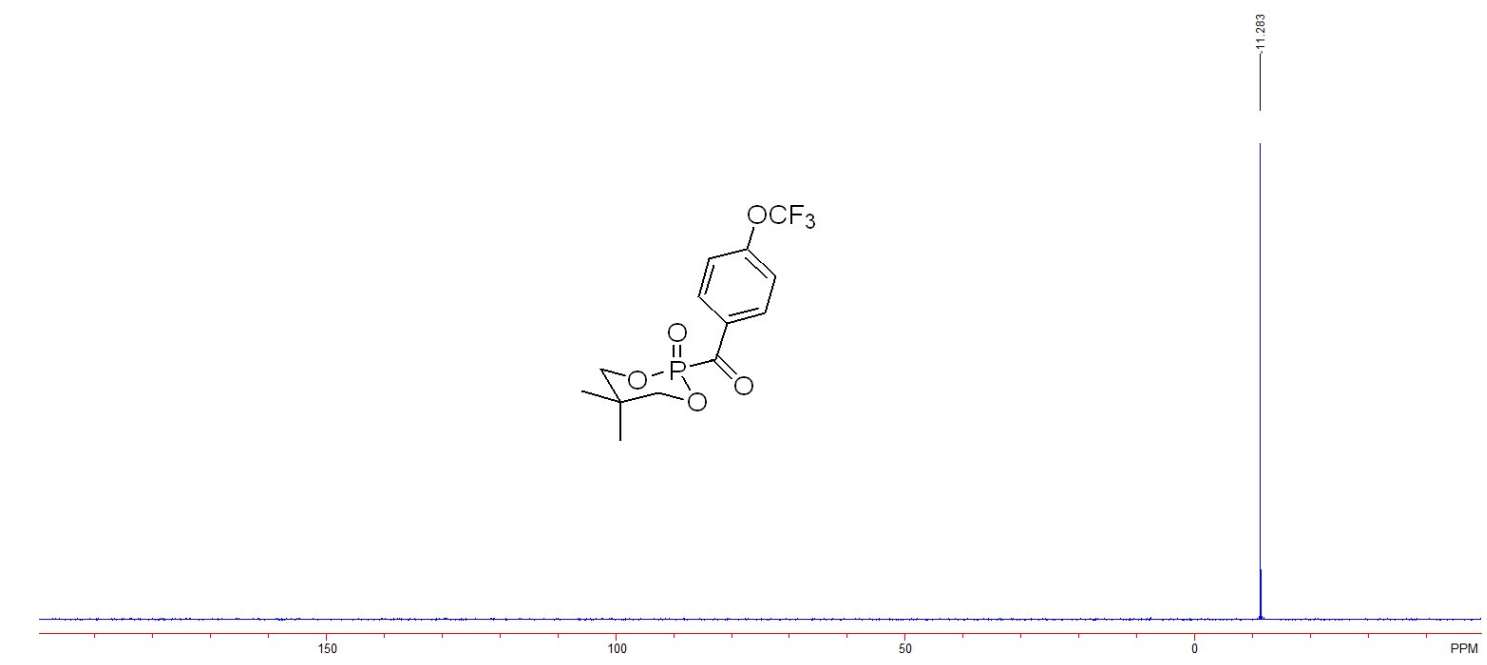




\section{Compound 5g}

${ }^{19} \mathrm{~F}$ NMR $\left(376 \mathrm{MHz}, \mathrm{CDCl}_{3}\right)$

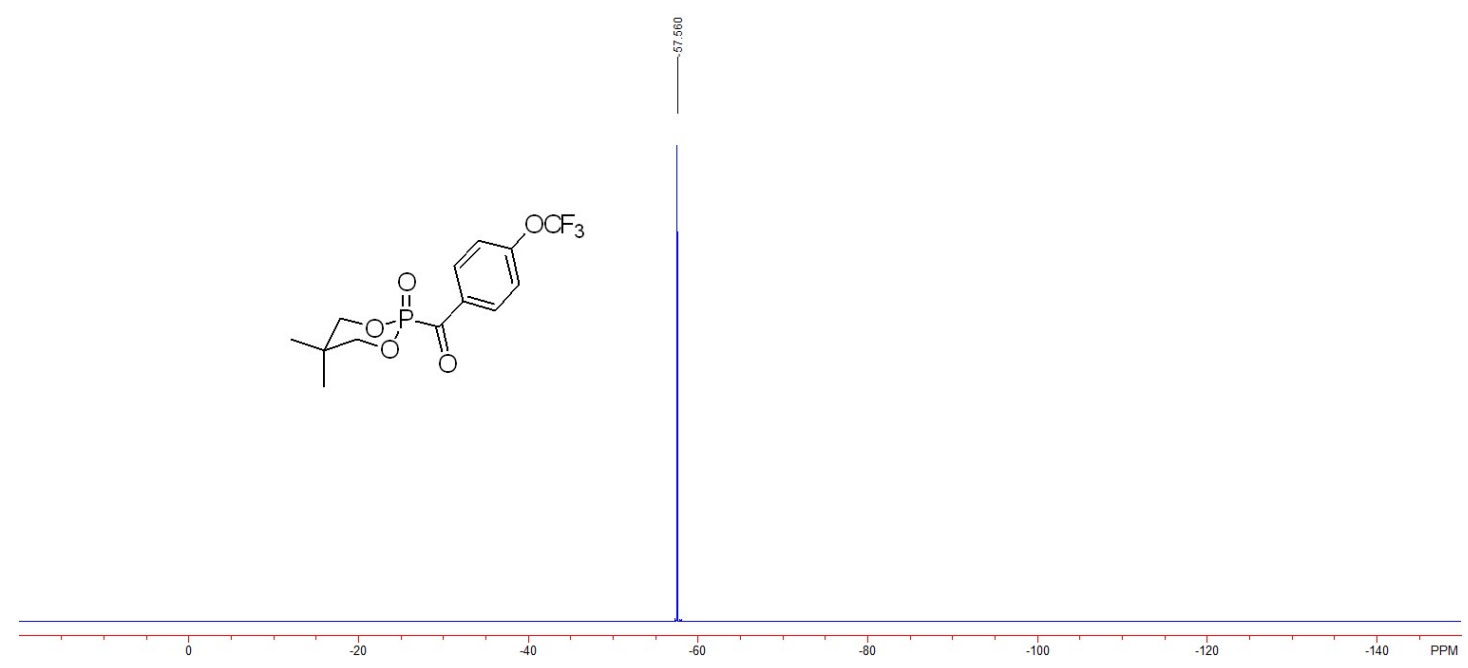

\section{Compound 5g}

${ }^{13} \mathrm{C} \mathrm{NMR}\left(101 \mathrm{MHz}, \mathrm{CDCl}_{3}\right)$

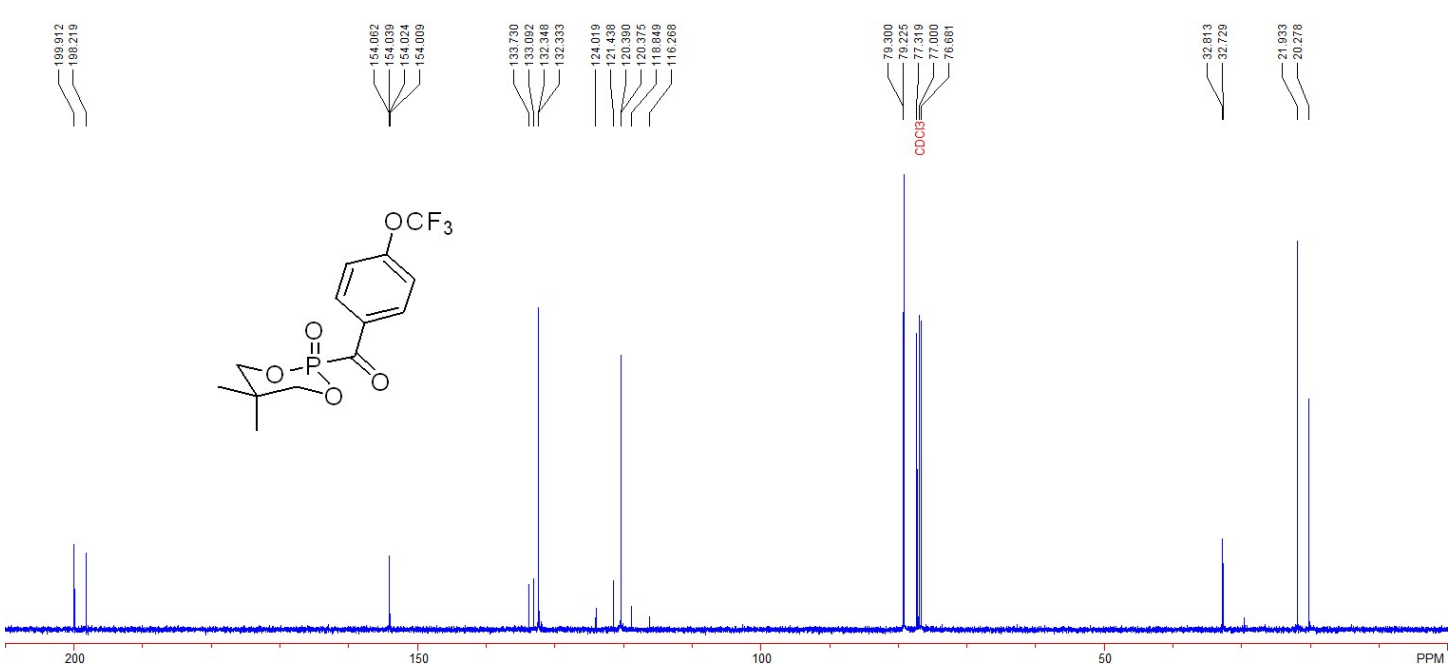


Compound 5h ${ }^{1} \mathrm{H}$ NMR (400 MHz, $\left.\mathrm{CDCl}_{3}\right)$

$|1|$

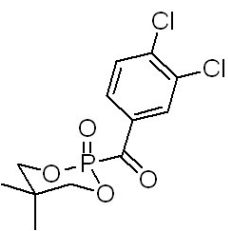

Compound 5h ${ }^{31} \mathrm{P}$ NMR (163 MHz, $\left.\mathrm{CDCl}_{3}\right)$

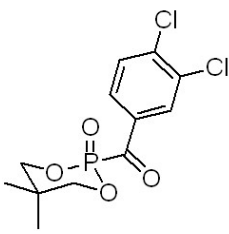

Compound 5h ${ }^{13} \mathrm{C}$ NMR (101 MHz, $\left.\mathrm{CDCl}_{3}\right)$
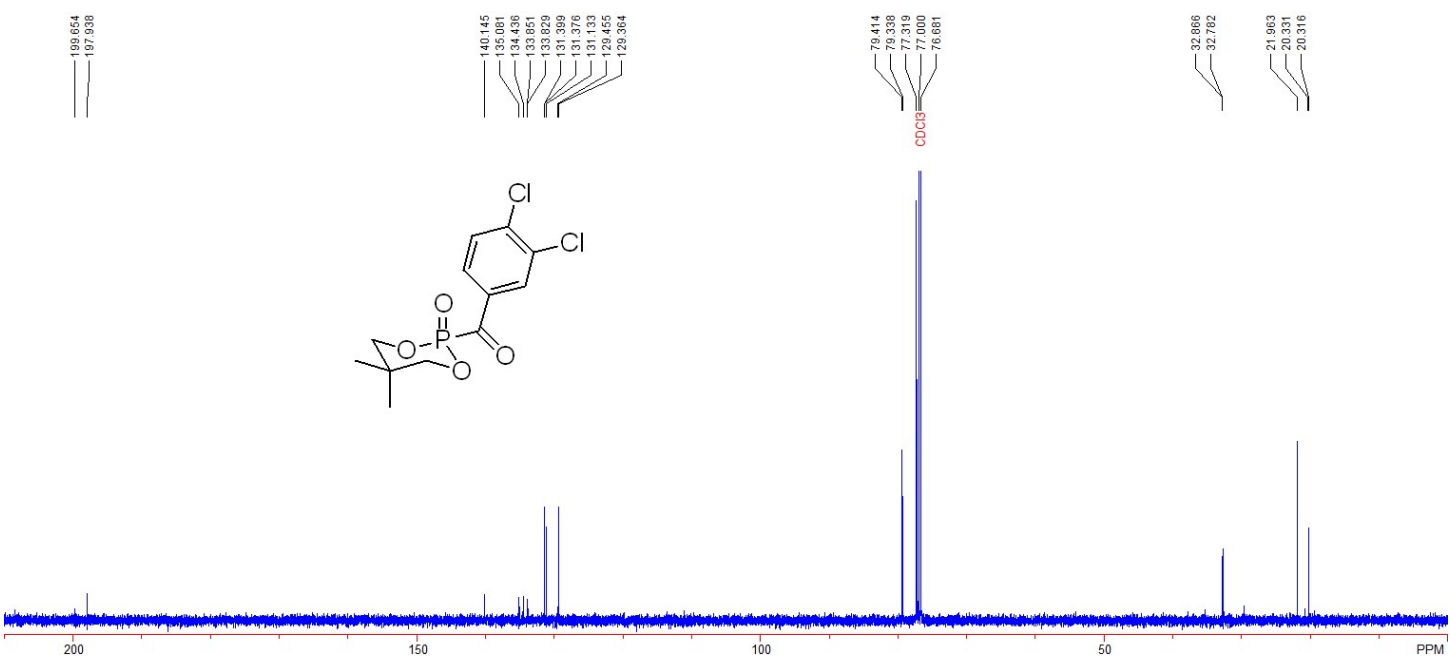
Compound 5i ${ }^{1} \mathrm{H}$ NMR (400 MHz, $\left.\mathrm{CDCl}_{3}\right)$

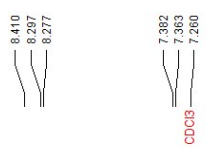

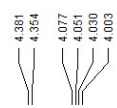
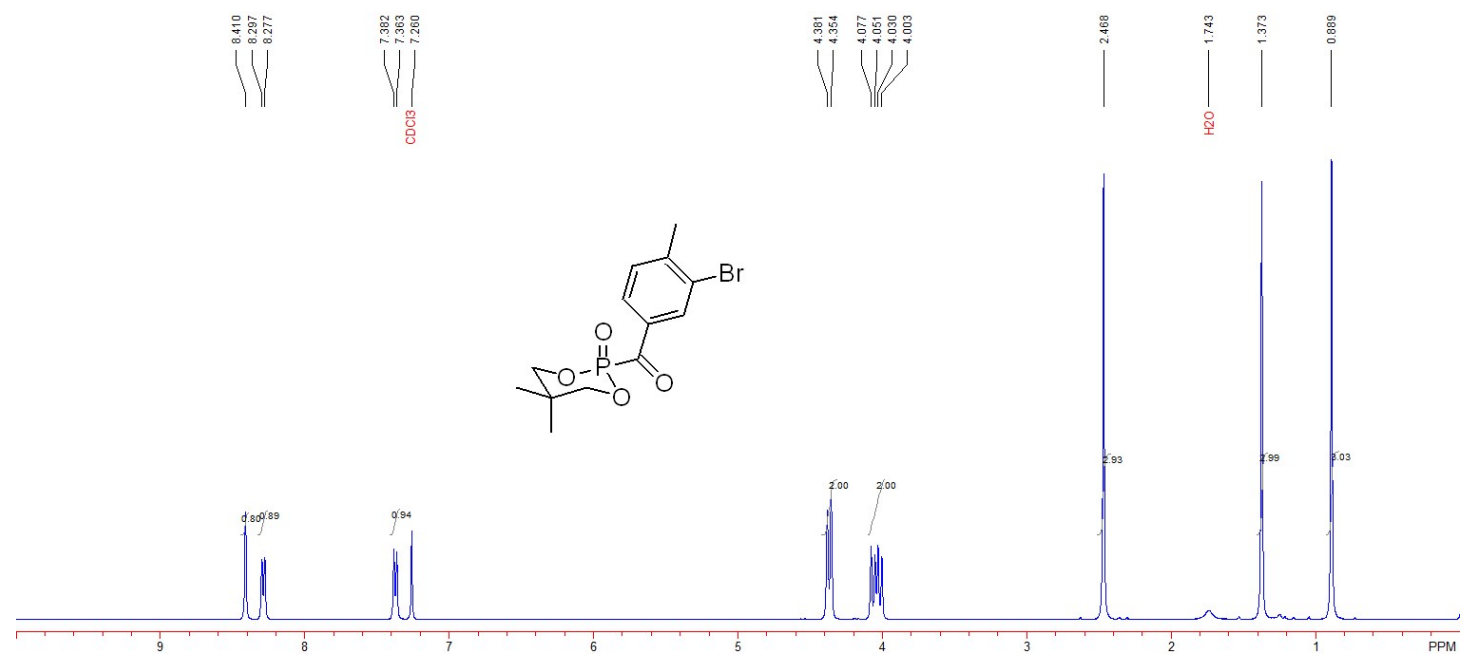

Compound 5i ${ }^{31} \mathrm{P}$ NMR (163 MHz, $\mathrm{CDCl}_{3}$ )

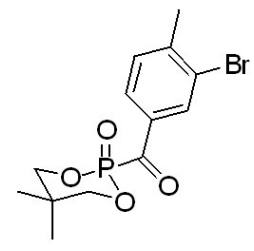

Compound 5i ${ }^{13} \mathrm{C}$ NMR (101 $\left.\mathrm{MHz}, \mathrm{CDCl}_{3}\right)$
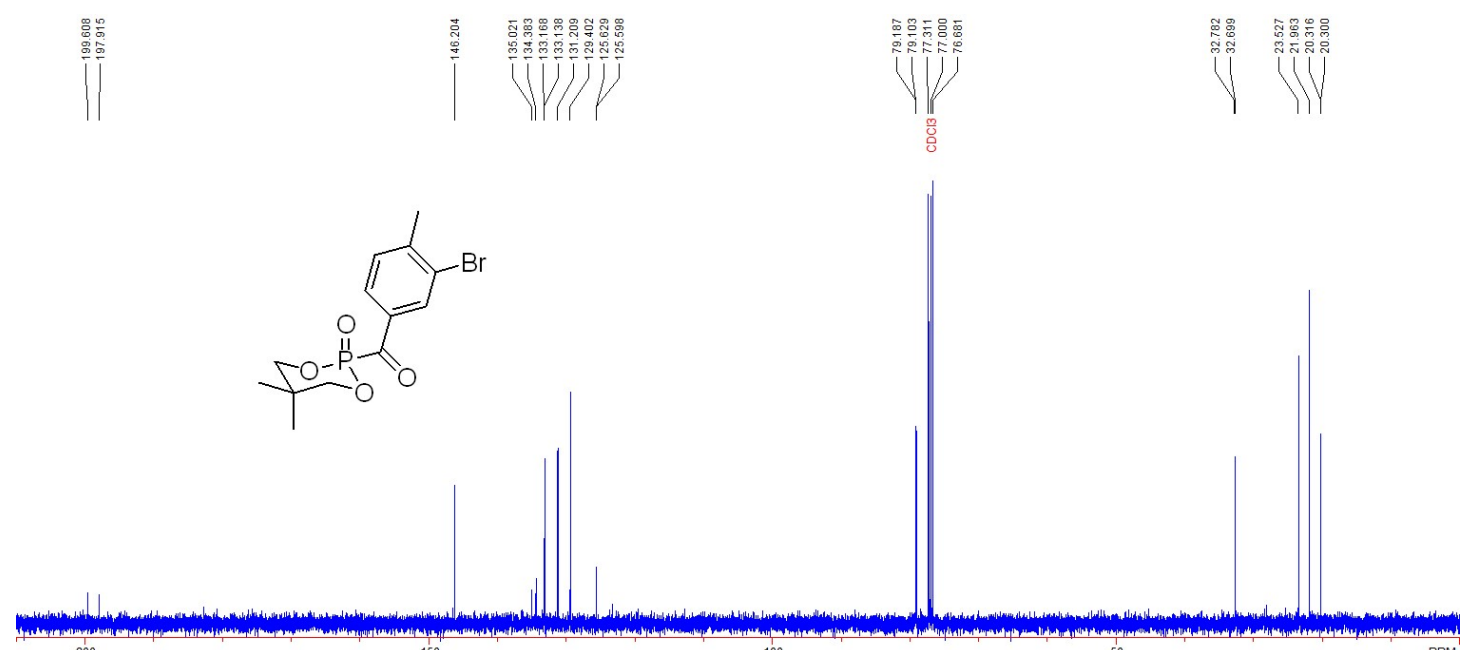
Compound 5j ${ }^{1} \mathrm{H}$ NMR (400 MHz, $\mathrm{CDCl}_{3}$ )

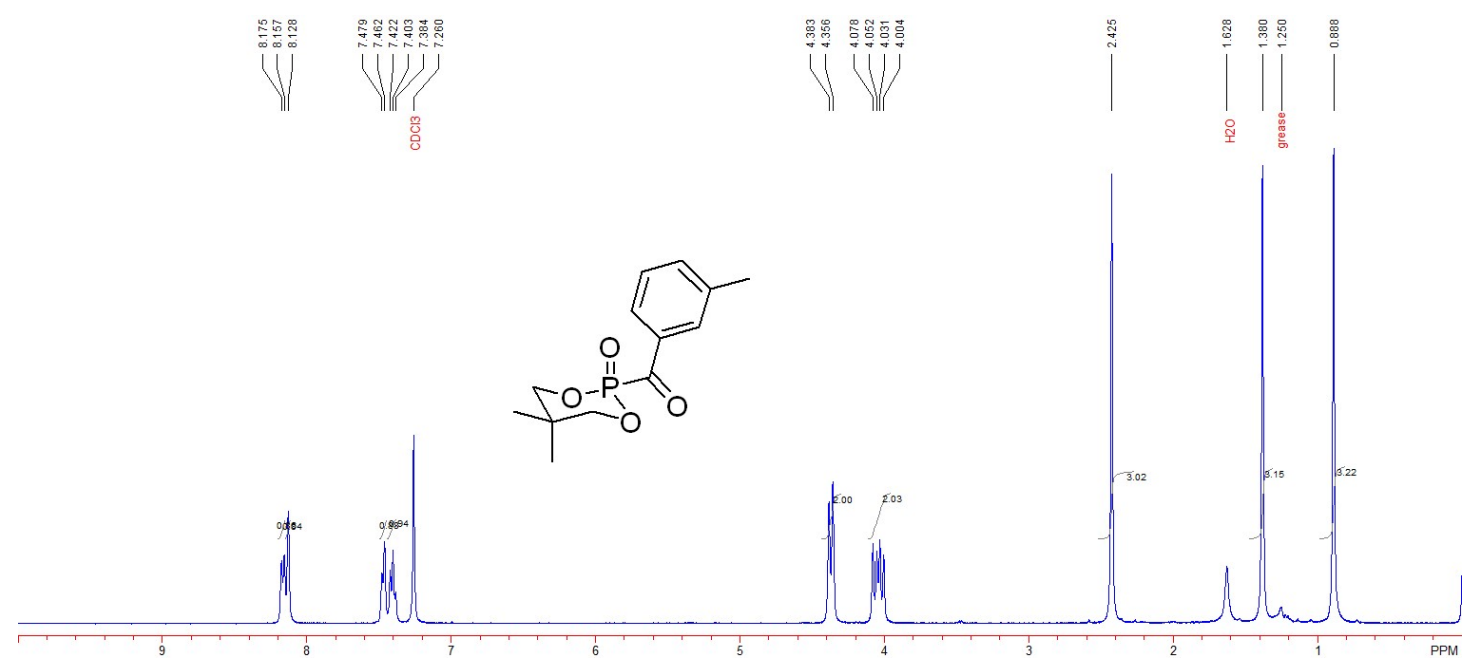

Compound 5j ${ }^{31} \mathrm{P}$ NMR (163 MHz, $\left.\mathrm{CDCl}_{3}\right)$

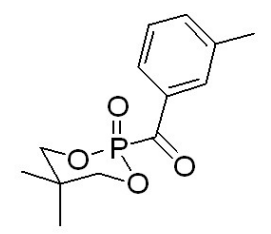

Compound 5j ${ }^{13} \mathrm{C}$ NMR (101 MHz, $\left.\mathrm{CDCl}_{3}\right)$

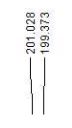

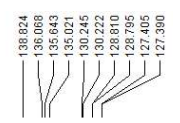
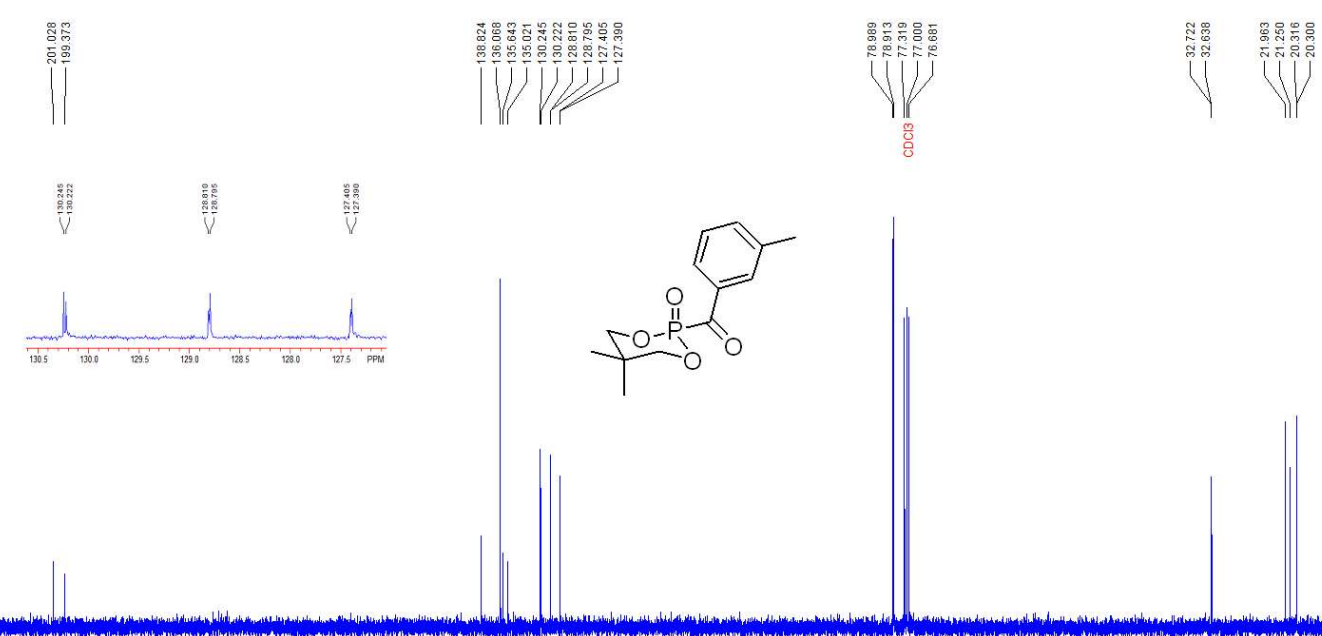
Compound 5k ${ }^{1} \mathrm{H}$ NMR (400 MHz, $\left.\mathrm{CDCl}_{3}\right)$

$\sqrt{1}$

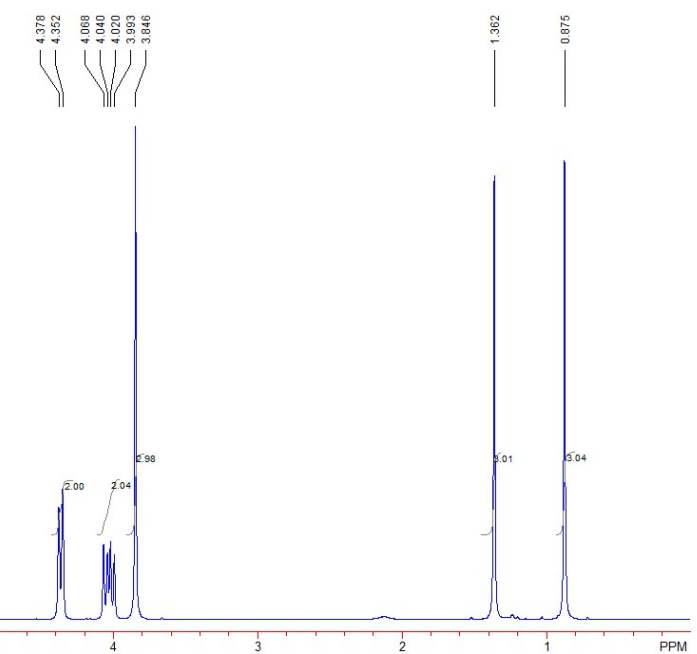

Compound 5k ${ }^{31} \mathrm{P}$ NMR (163 MHz, CDCl3)

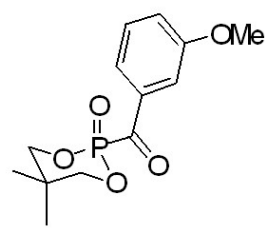

Compound 5k ${ }^{13} \mathrm{C}$ NMR (101 MHz, $\left.\mathrm{CDCl}_{3}\right)$
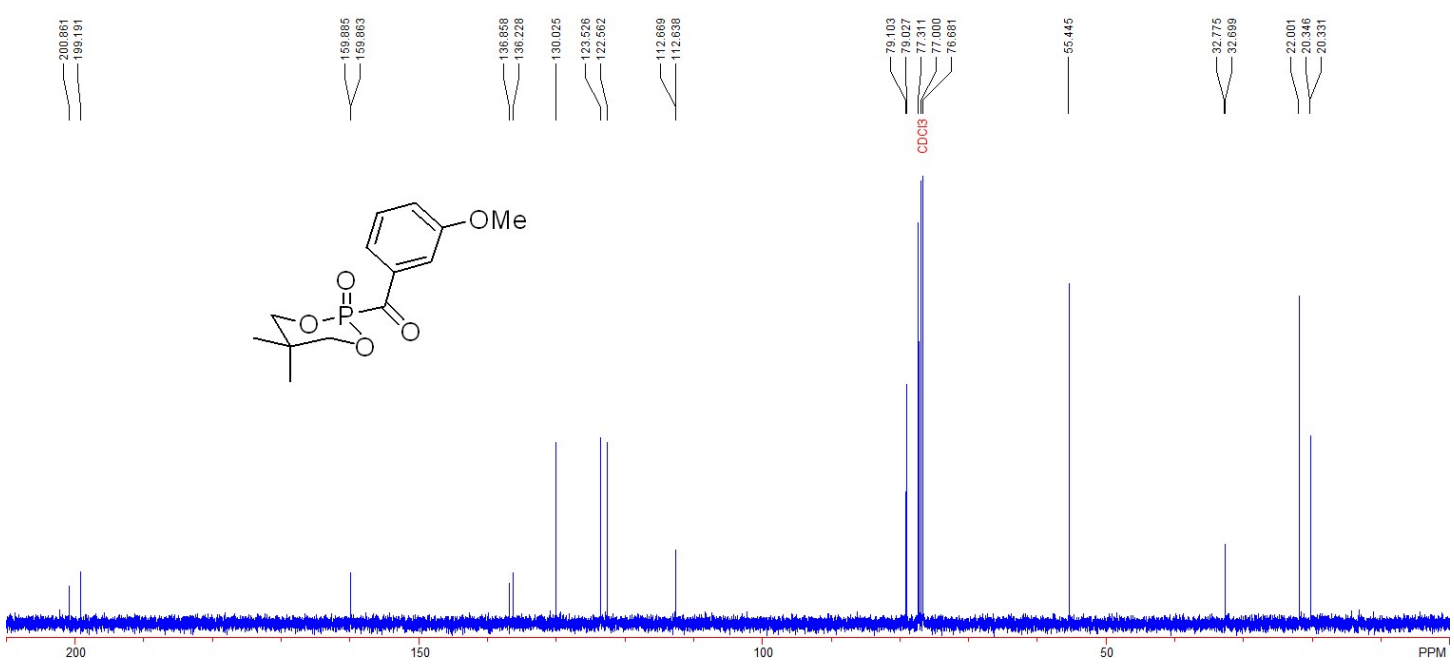
Compound $5 \mathbf{l}{ }^{1} \mathrm{H}$ NMR (400 MHz, $\left.\mathrm{CDCl}_{3}\right)$
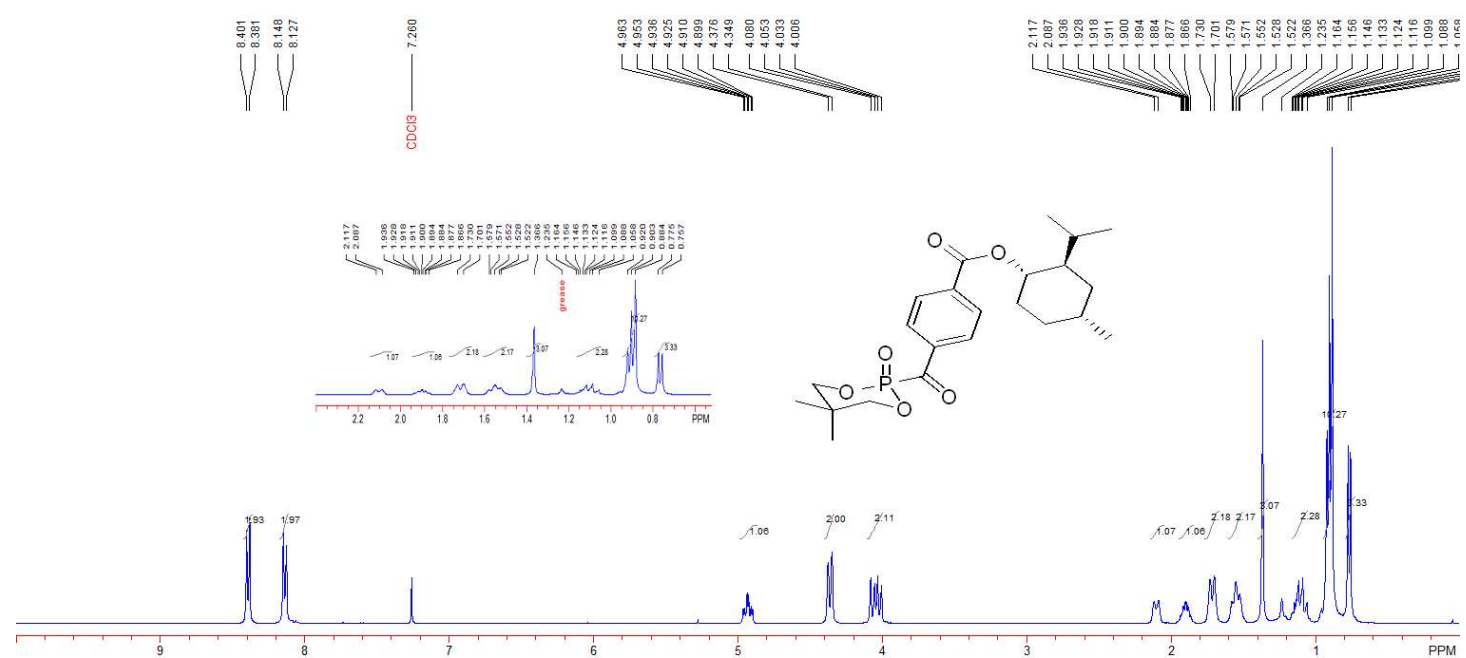

Compound 5l ${ }^{31} \mathrm{P}$ NMR (163 MHz, $\left.\mathrm{CDCl}_{3}\right)$

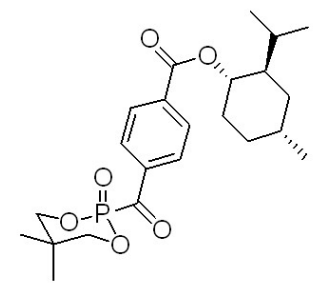

Compound 5l ${ }^{13} \mathrm{C}$ NMR (101 $\left.\mathrm{MHz}, \mathrm{CDCl}_{3}\right)$
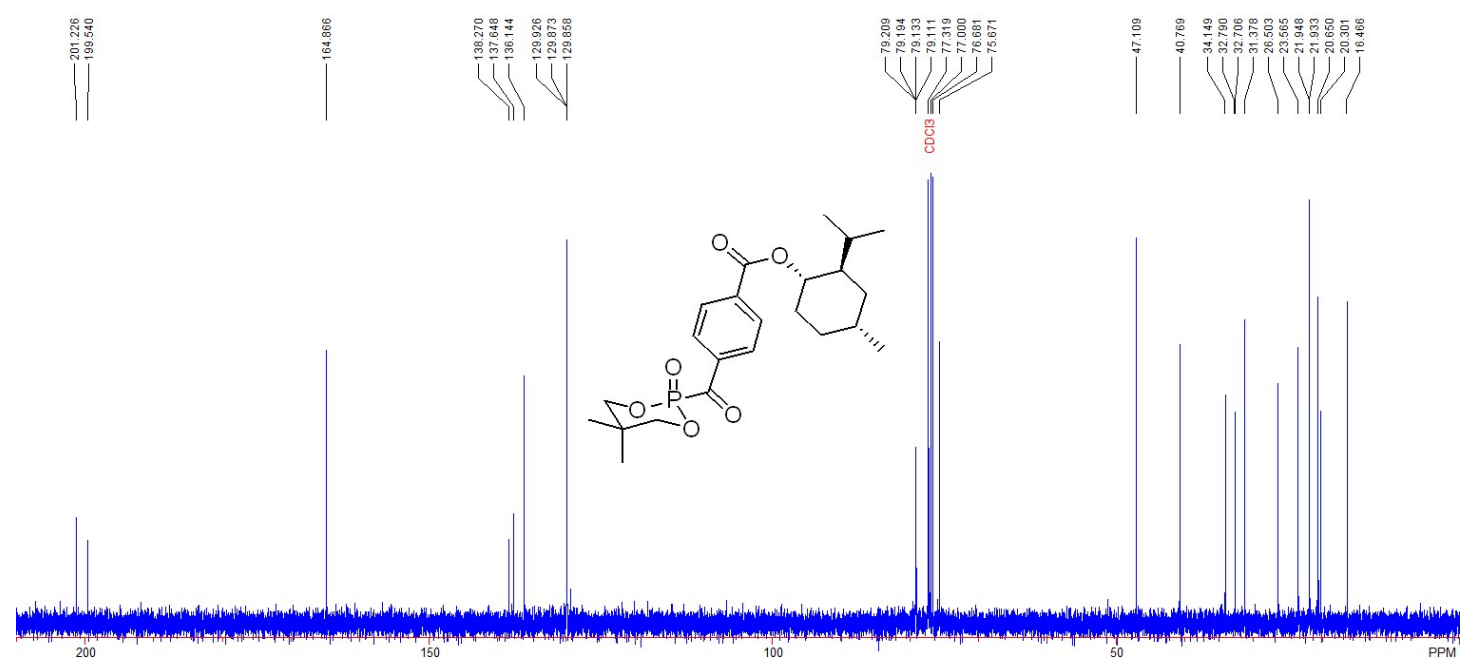
Compound 5m ${ }^{1} \mathrm{H}$ NMR (400 MHz, $\left.\mathrm{CDCl}_{3}\right)$

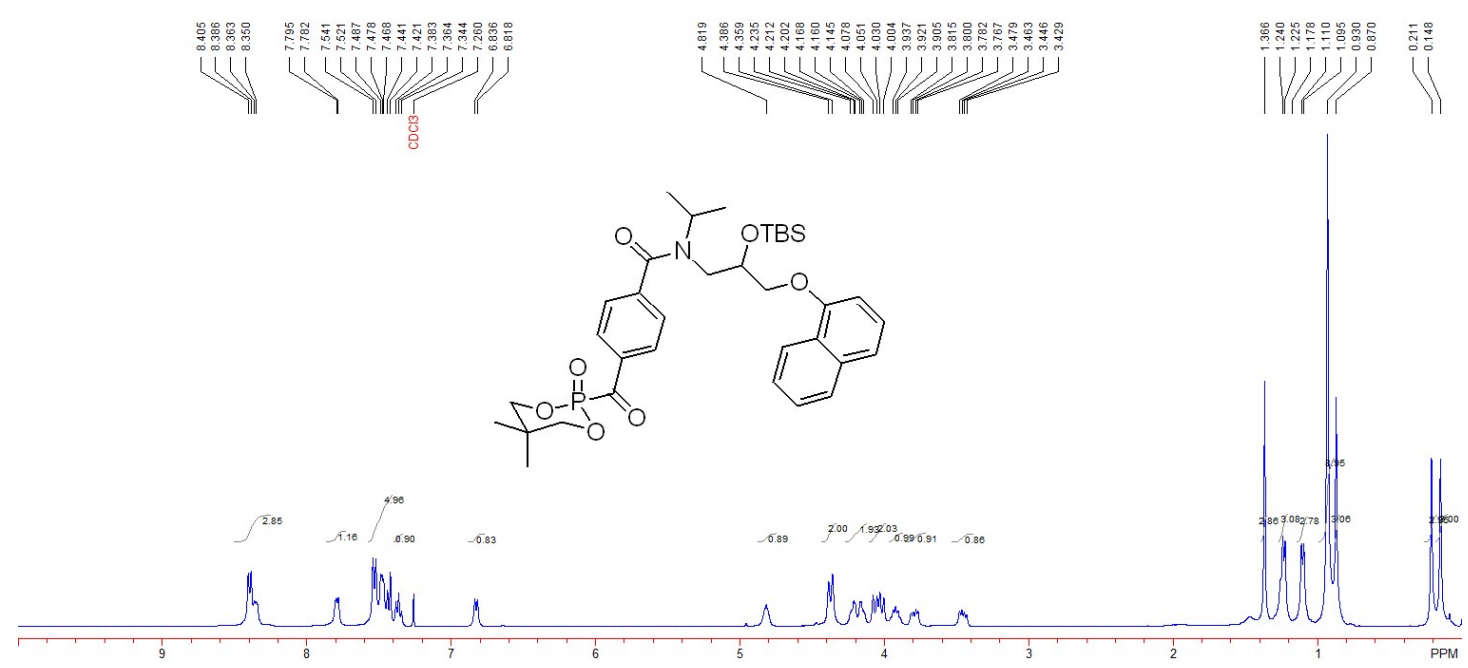

Compound 5m ${ }^{31} \mathrm{P}$ NMR (163 MHz, $\left.\mathrm{CDCl}_{3}\right)$

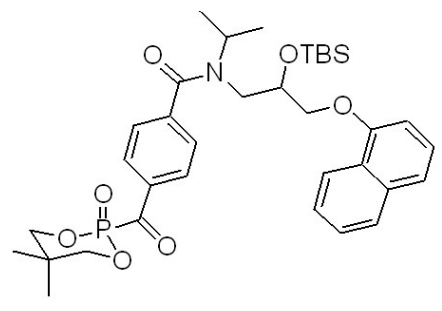

Compound 5m ${ }^{13} \mathrm{C}$ NMR (101 MHz, $\left.\mathrm{CDCl}_{3}\right)$

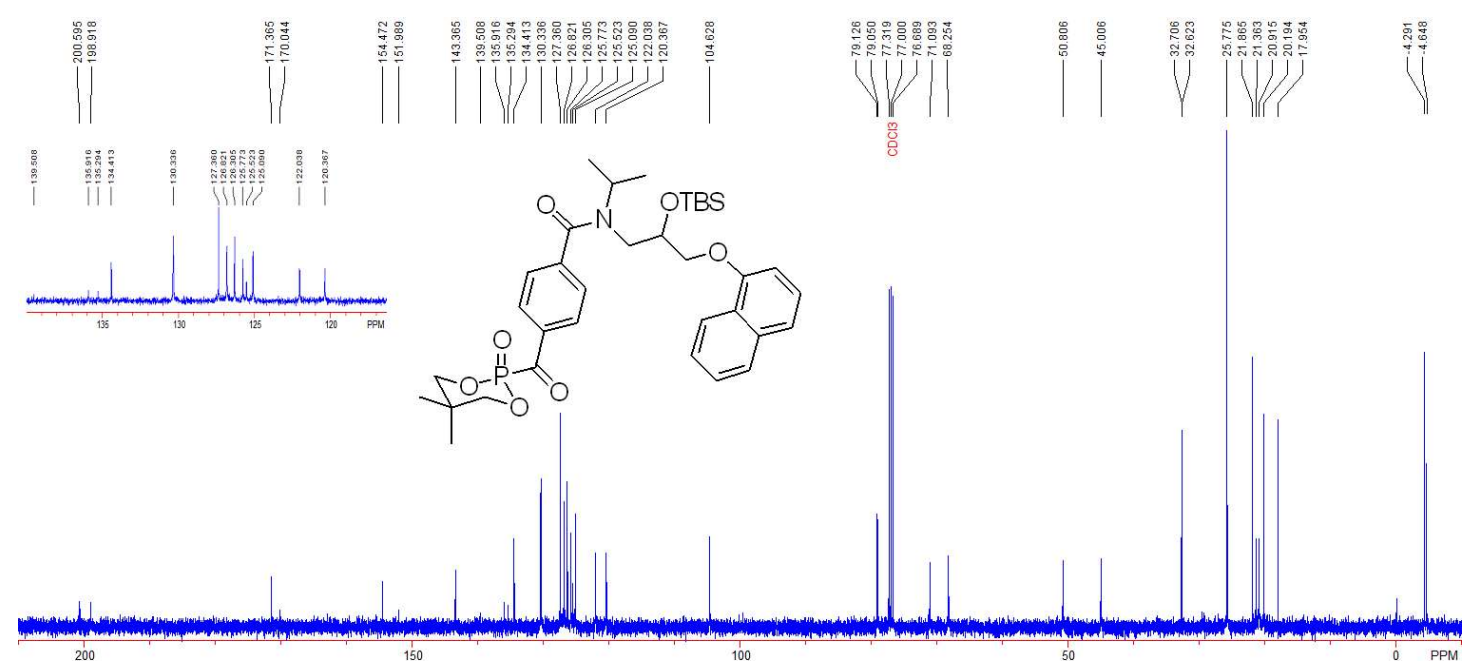


Compound 5n ${ }^{1} \mathrm{H}$ NMR (400 MHz, $\left.\mathrm{CDCl}_{3}\right)$ :

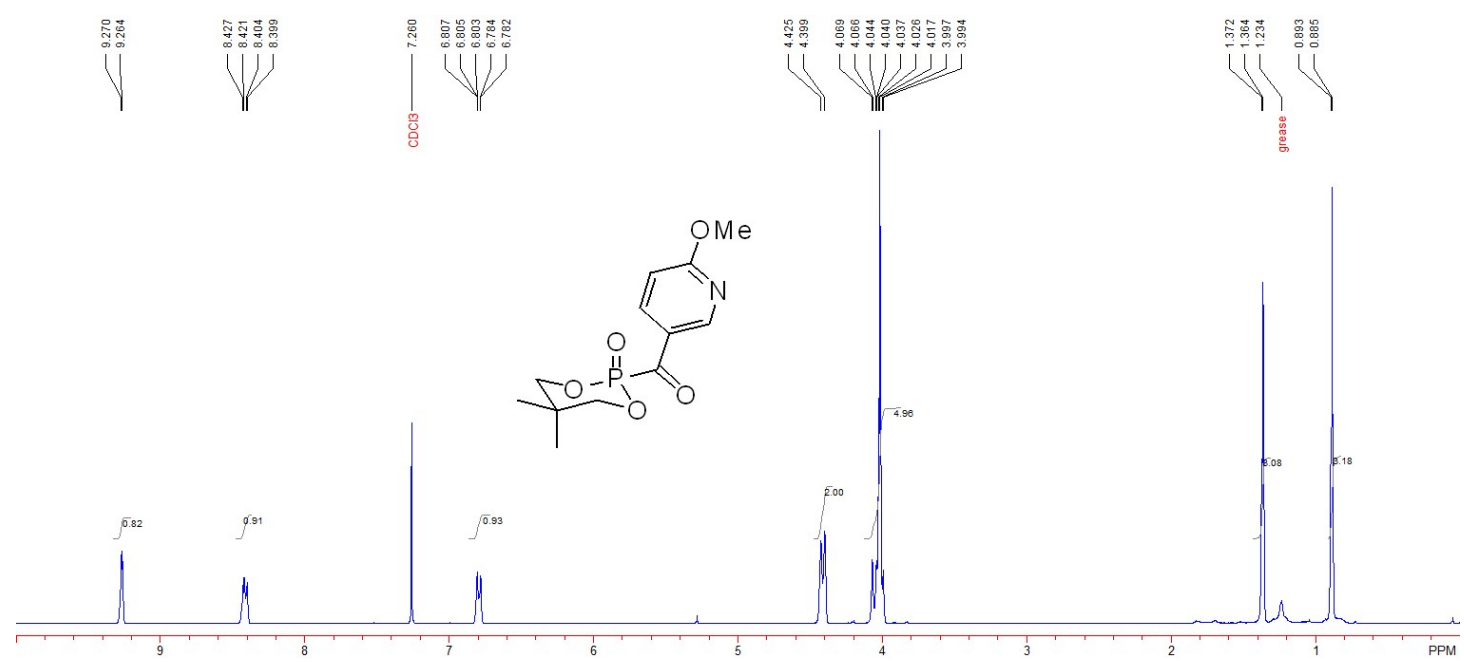

Compound 5n ${ }^{31} \mathrm{P}$ NMR (163 MHz, $\left.\mathrm{CDCl}_{3}\right)$

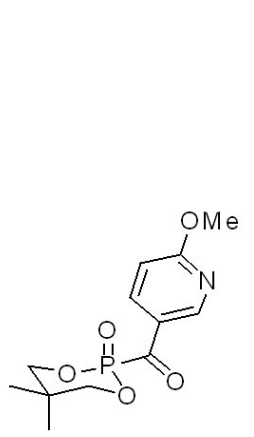

Compound 5n ${ }^{13} \mathrm{C}$ NMR (101 MHz, $\left.\mathrm{CDCl}_{3}\right)$
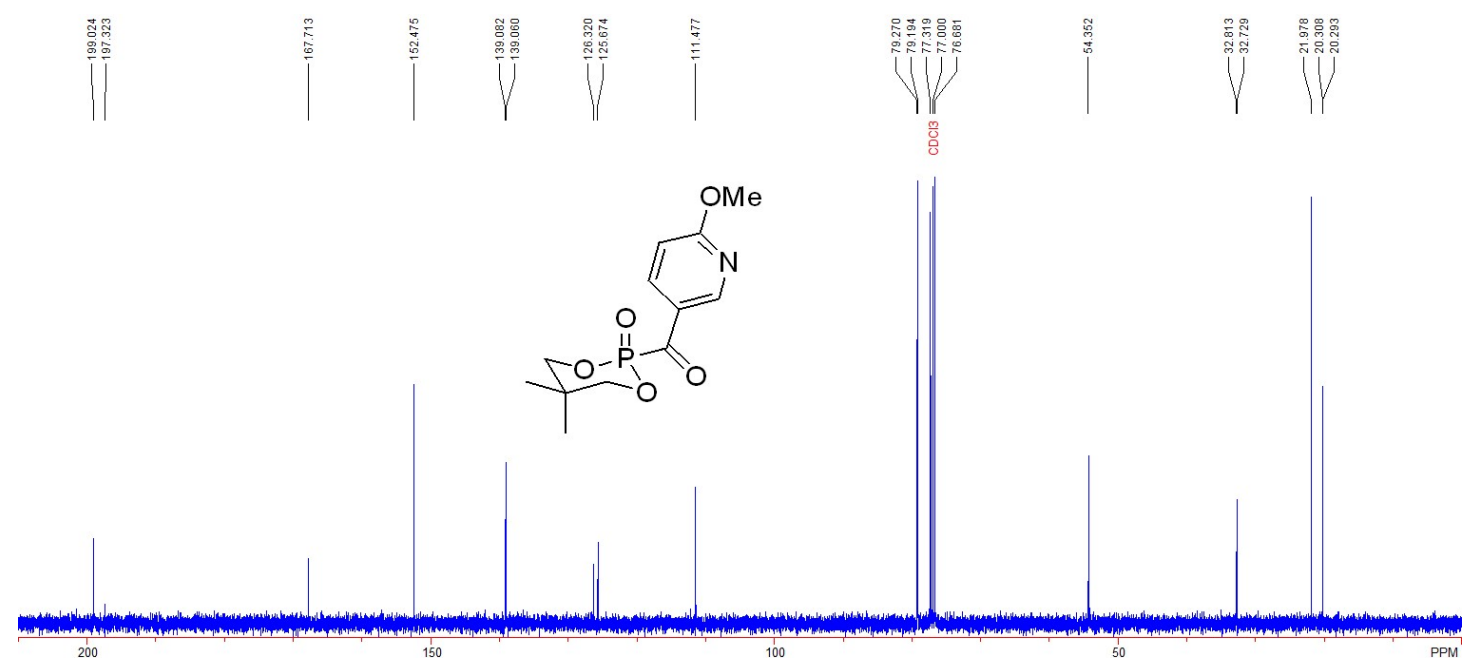
Compound $50{ }^{1} \mathrm{H}$ NMR (400 MHz, $\left.\mathrm{CDCl}_{3}\right)$

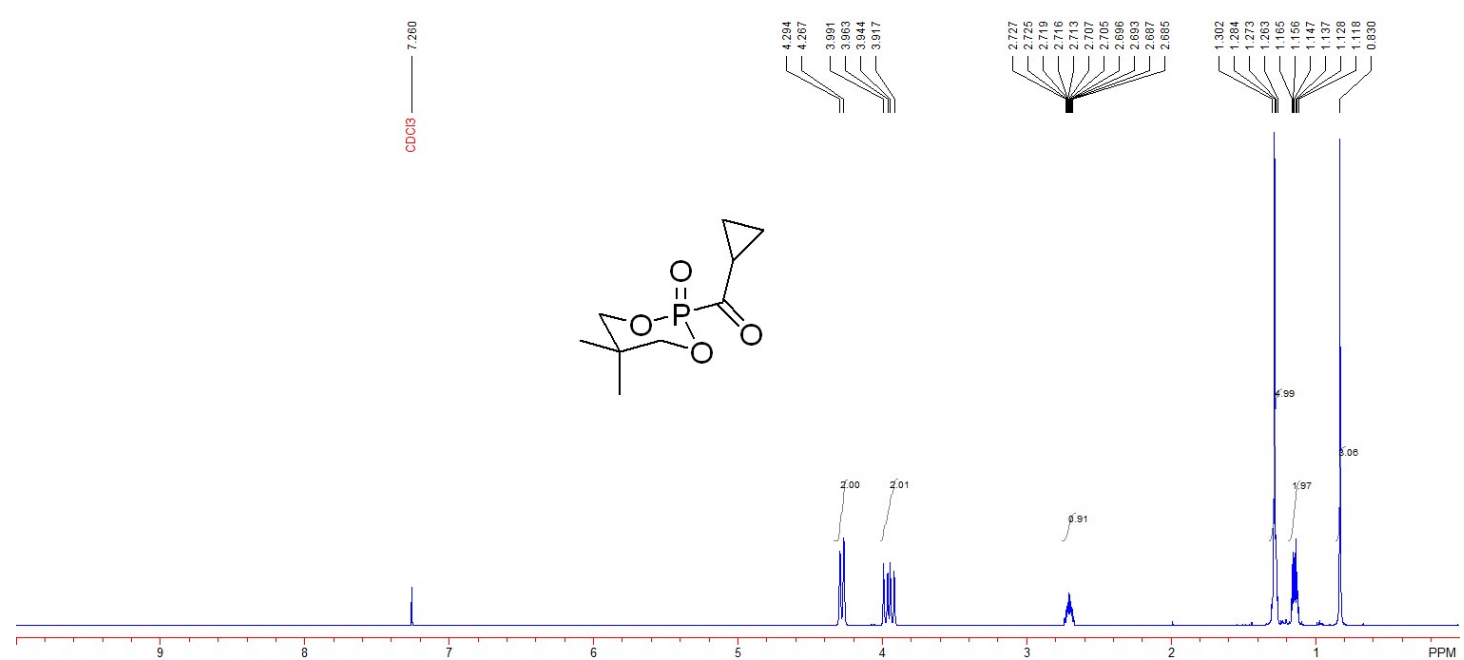

Compound 5o ${ }^{31} \mathrm{P}$ NMR (163 MHz, $\left.\mathrm{CDCl}_{3}\right)$

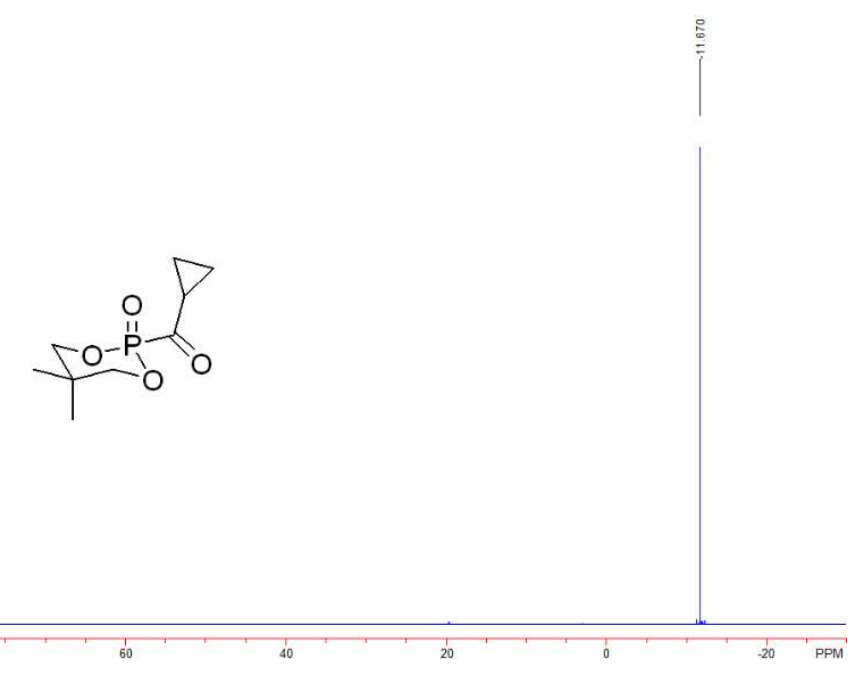

Compound 5o ${ }^{13} \mathrm{C}$ NMR (101 MHz, $\left.\mathrm{CDCl}_{3}\right)$

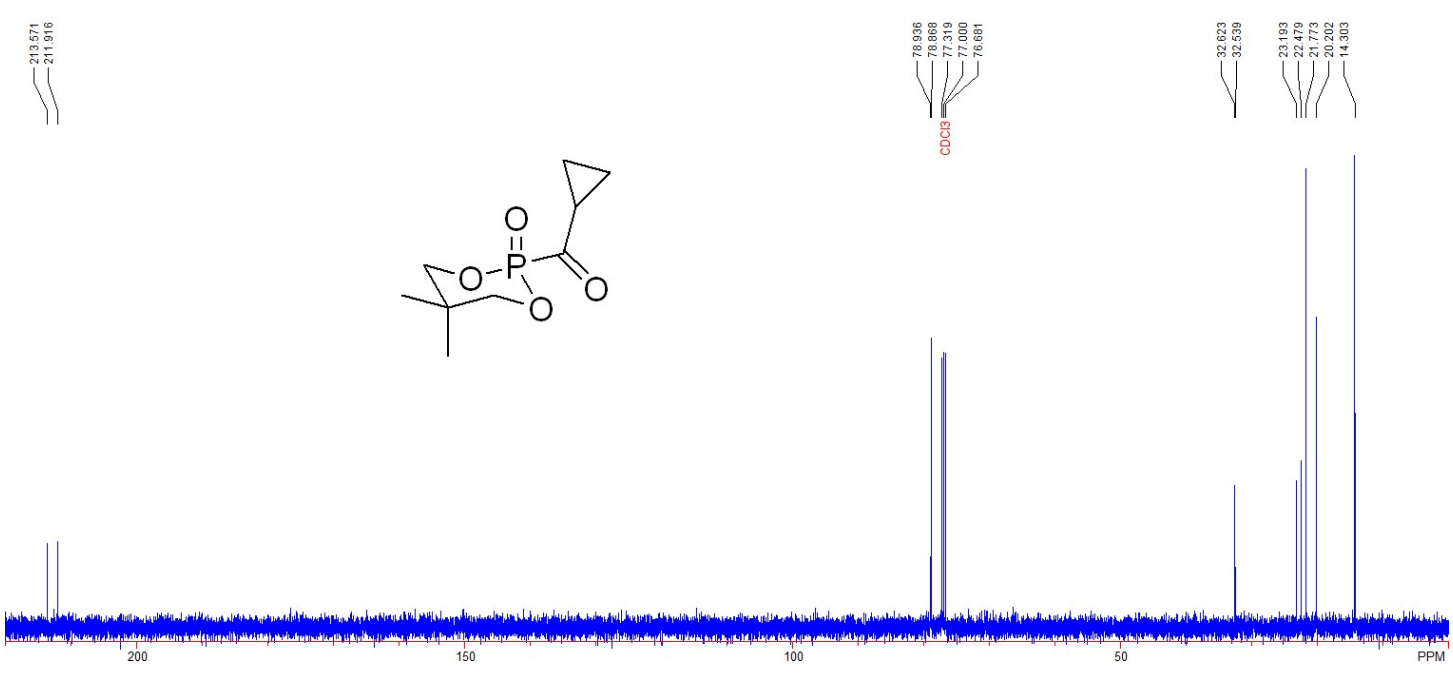


Compound 5p ${ }^{1} \mathrm{H}$ NMR $\left(400 \mathrm{MHz}, \mathrm{CDCl}_{3}\right)$

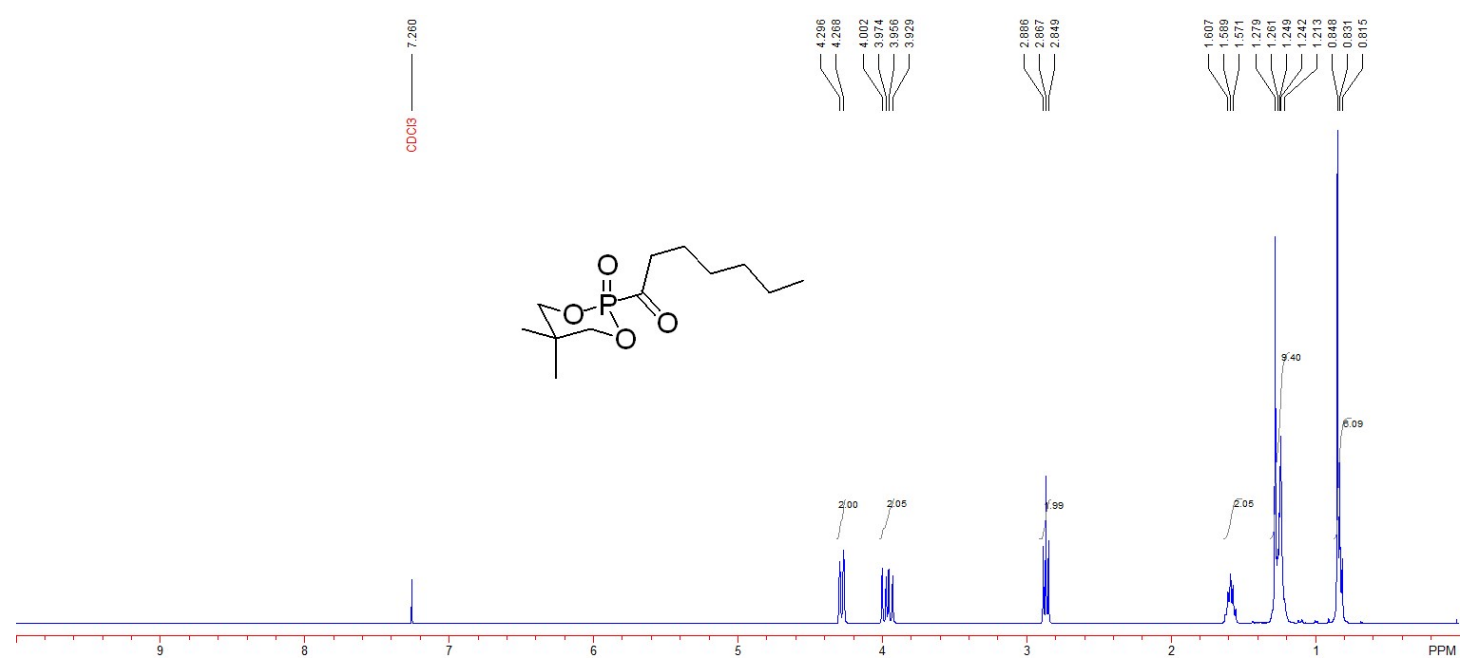

Compound 5p ${ }^{31} \mathrm{P}$ NMR (163 MHz, $\left.\mathrm{CDCl}_{3}\right)$

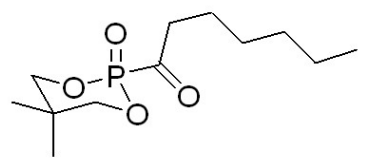

Compound 5p ${ }^{13} \mathrm{C}$ NMR (101 MHz, $\left.\mathrm{CDCl}_{3}\right)$
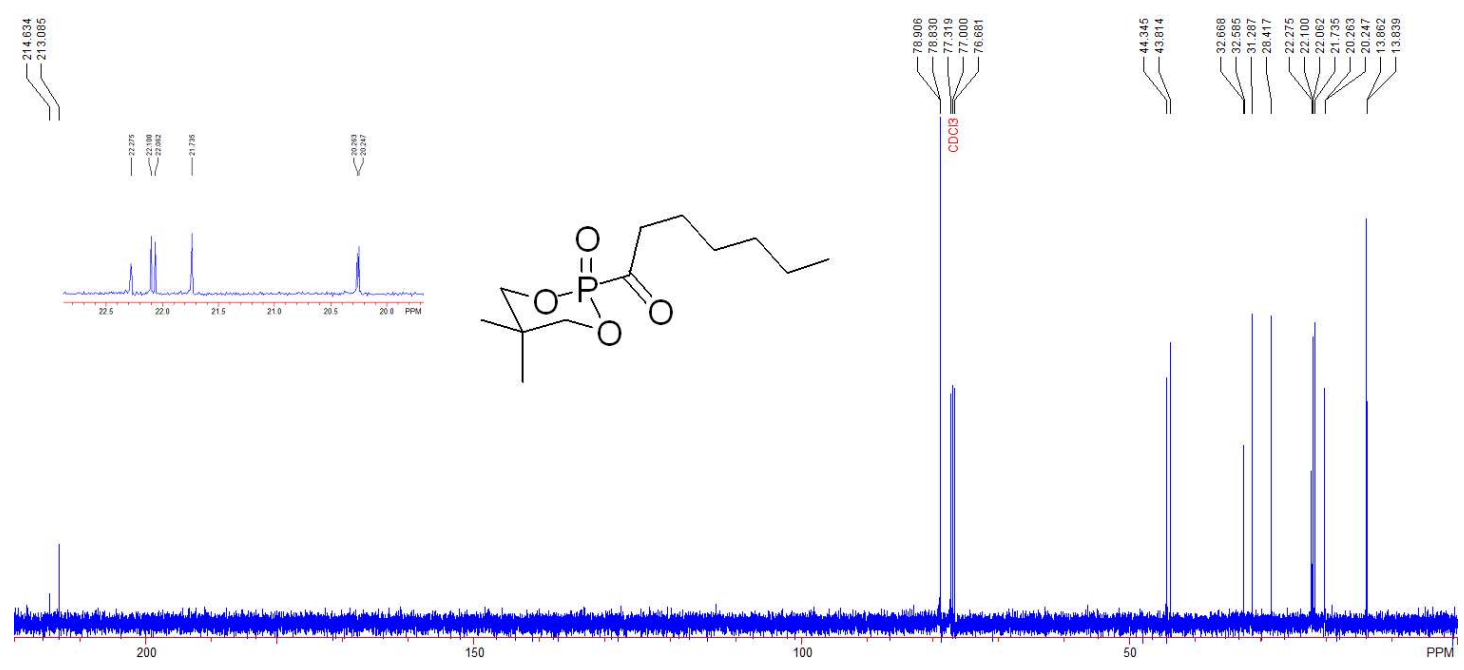
Compound 3i ${ }^{1} \mathrm{H}$ NMR (400 $\mathrm{MHz}, \mathrm{CDCl}_{3}$ )

U
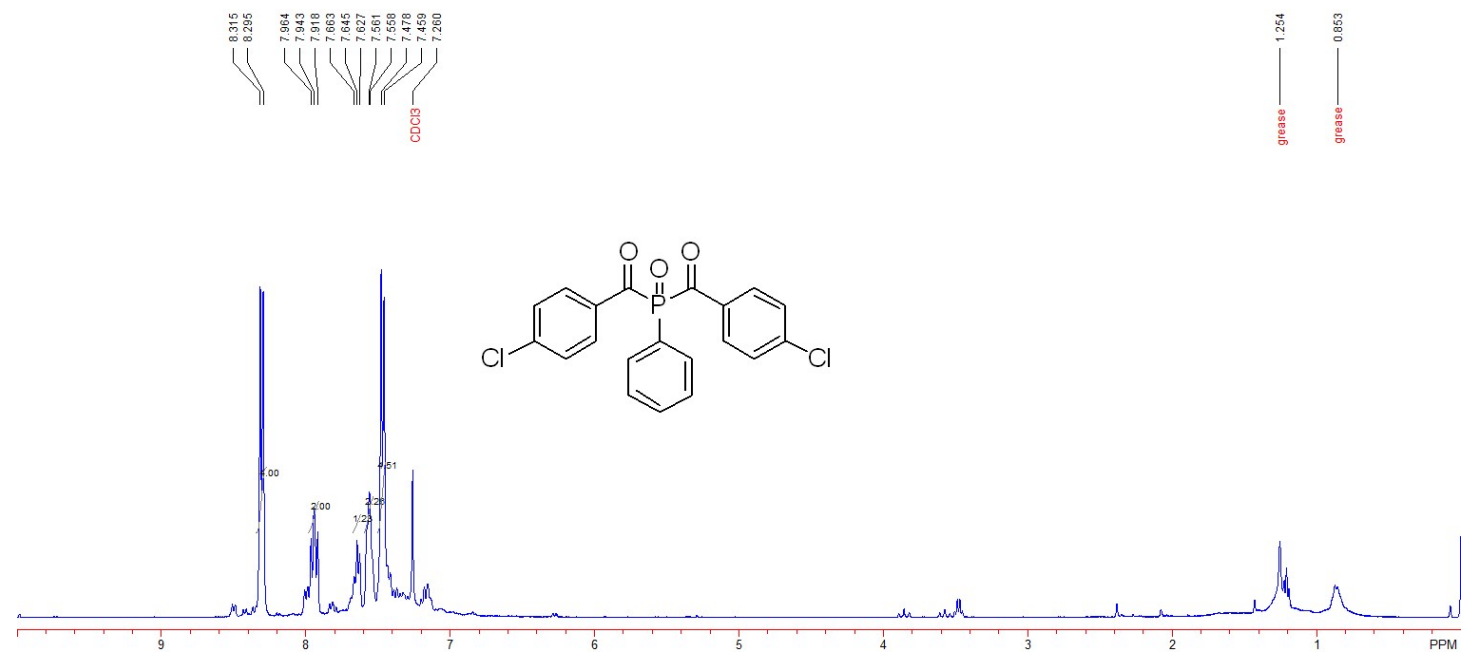

Compound $3 \mathbf{i}^{31} \mathrm{P}$ NMR (163 MHz, $\mathrm{CDCl}_{3}$ )

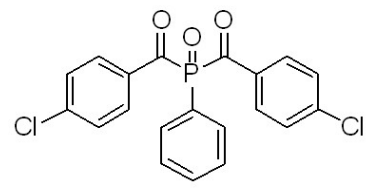

Compound $3 \mathbf{i}^{13} \mathrm{C}$ NMR (125 MHz, $\left.\mathrm{CDCl}_{3}\right)$

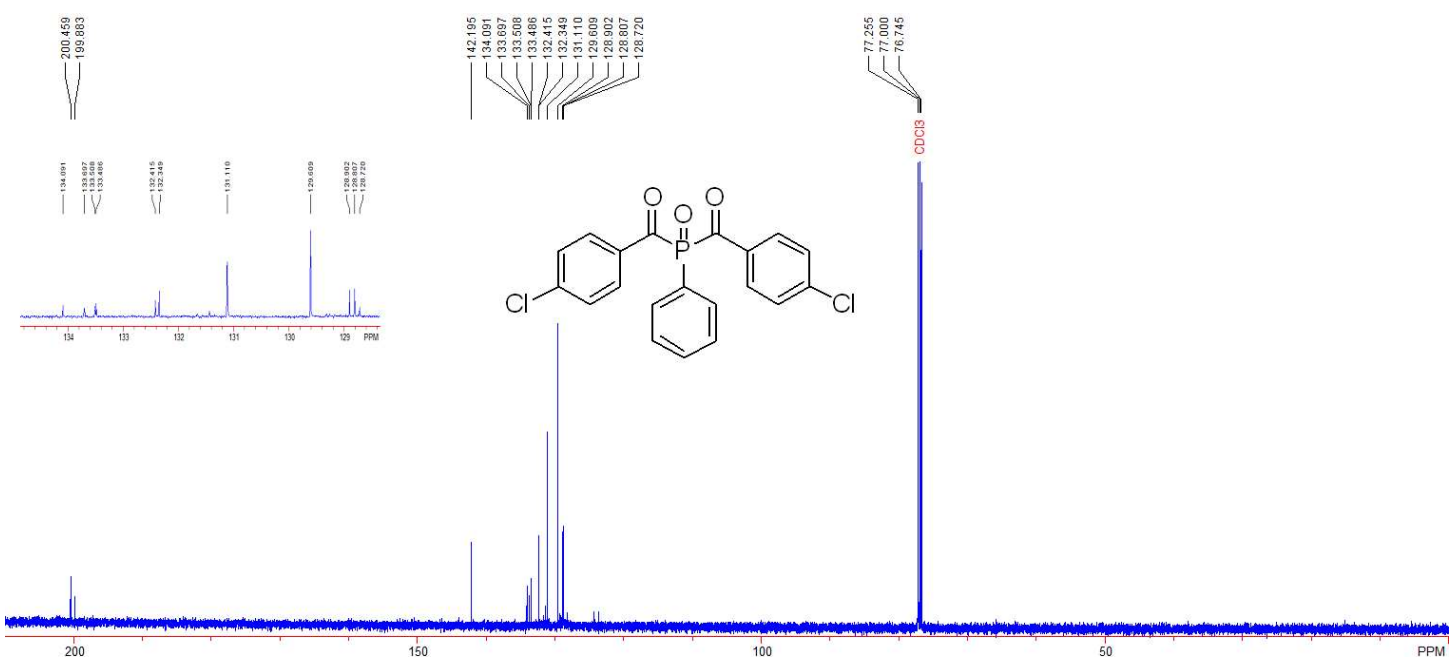


6. ${ }^{1} \mathrm{H},{ }^{13} \mathrm{C}$ and ${ }^{19} \mathrm{~F}$ NMR spectra of all products

Compound 4a ${ }^{1} \mathrm{H}$ NMR $\left(400 \mathrm{MHz}, \mathrm{CDCl}_{3}\right)$

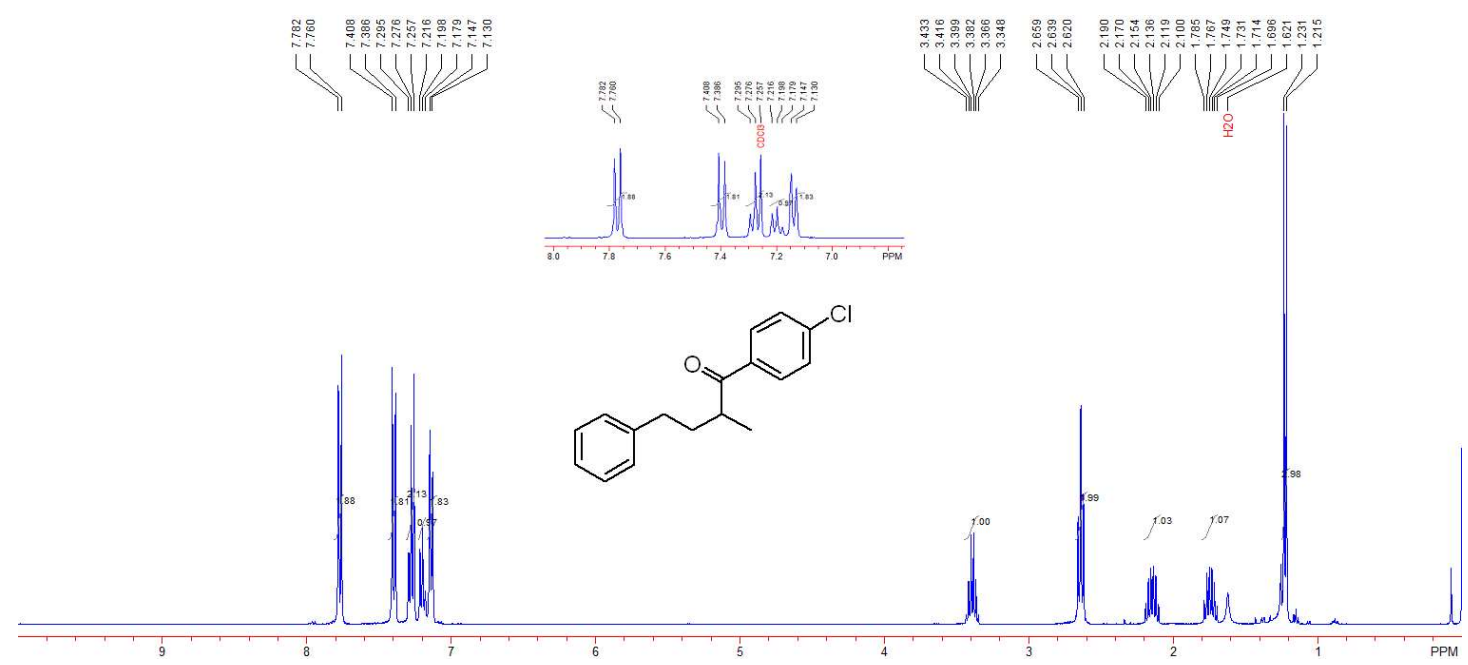

Compound 4a ${ }^{13} \mathrm{C}$ NMR (101 MHz, $\left.\mathrm{CDCl}_{3}\right)$
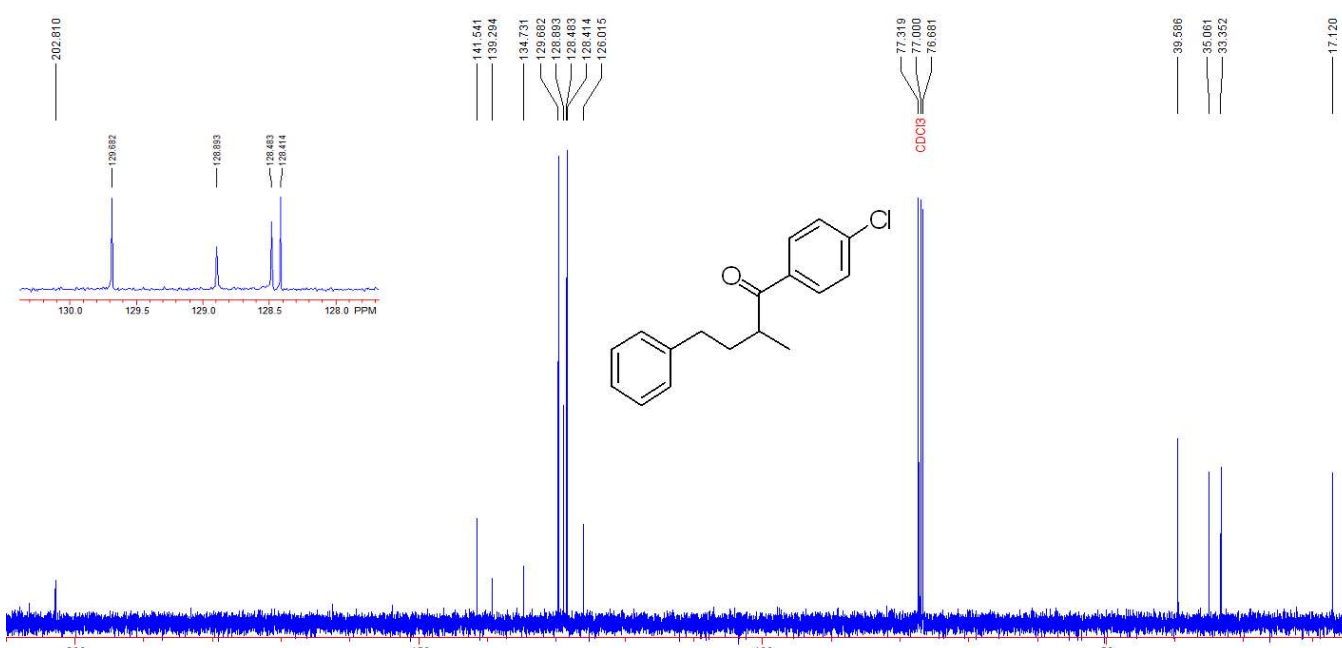


\section{Compound 4b}

${ }^{1} \mathrm{H} \mathrm{NMR}\left(400 \mathrm{MHz}, \mathrm{CDCl}_{3}\right)$

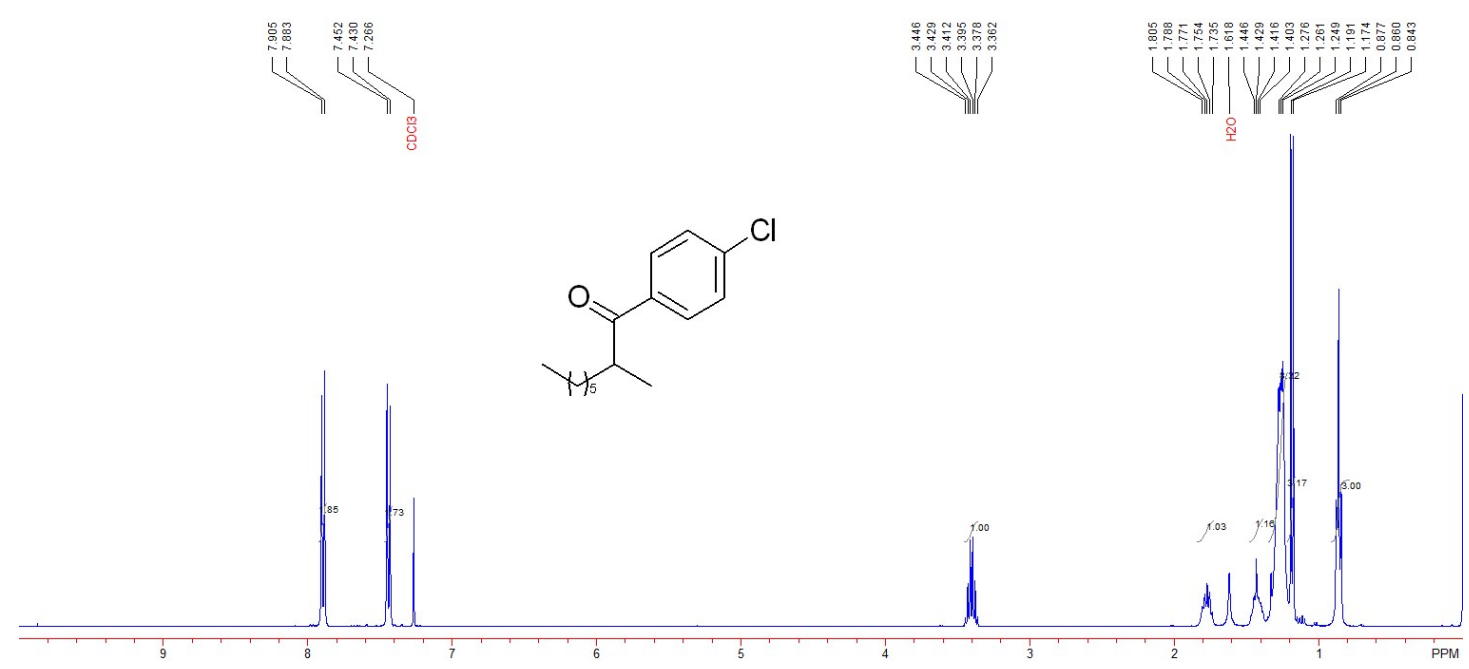

\section{Compound 4b}

${ }^{13} \mathrm{C} \mathrm{NMR}\left(101 \mathrm{MHz}, \mathrm{CDCl}_{3}\right)$

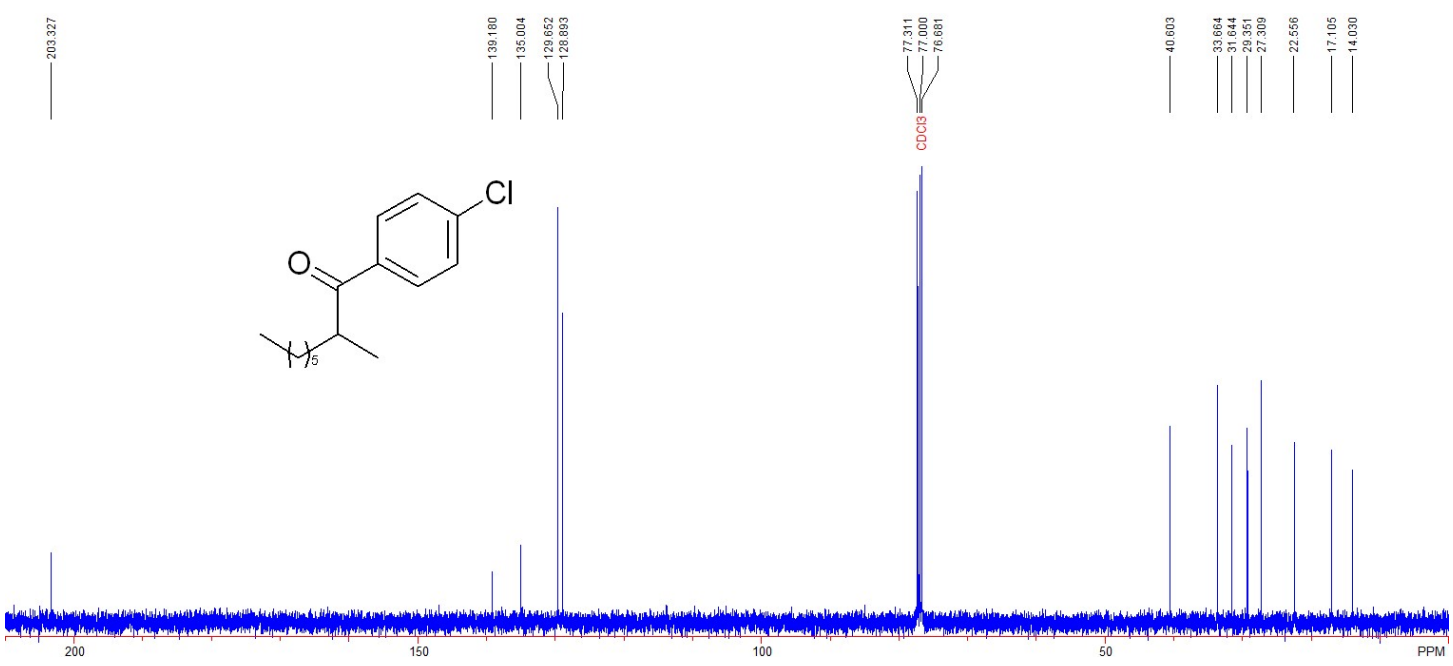




\section{Compound 4c}

${ }^{1} \mathrm{H}$ NMR (400 MHz, $\left.\mathrm{CDCl}_{3}\right)$

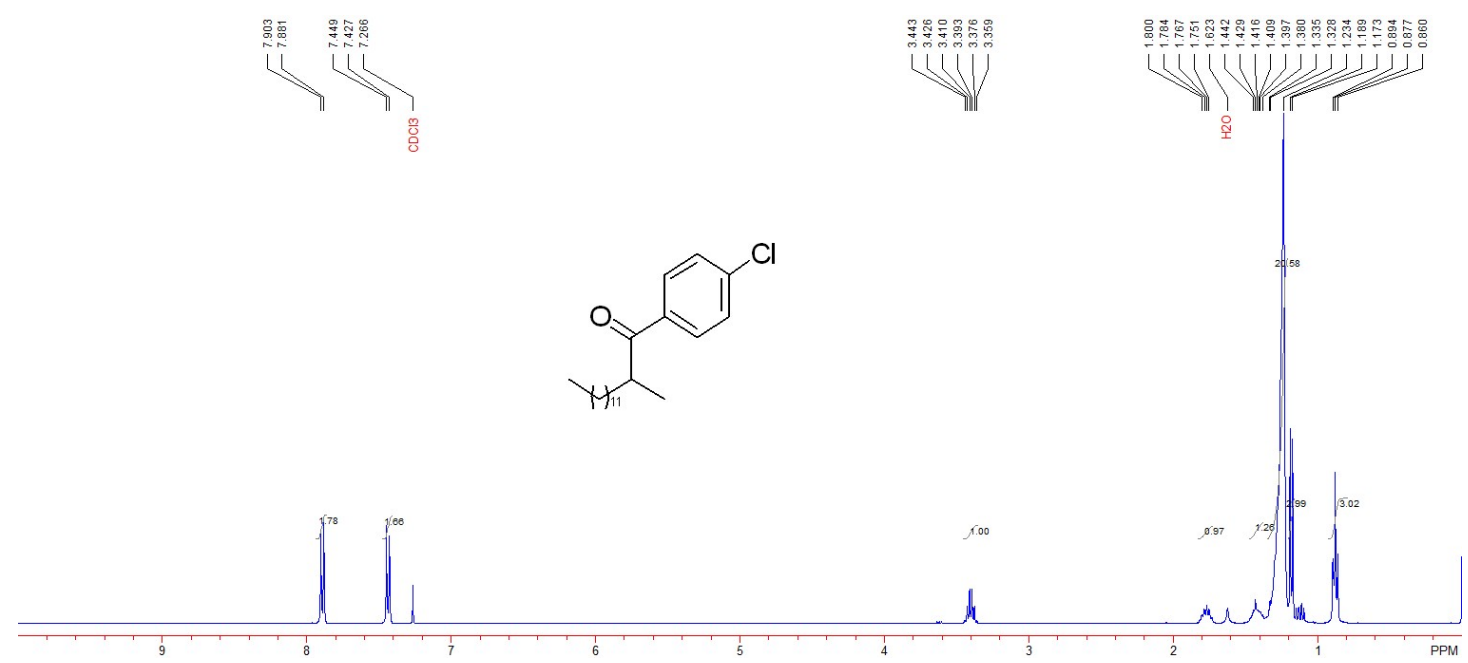

\section{Compound 4c}

${ }^{13} \mathrm{C} \mathrm{NMR}\left(101 \mathrm{MHz}, \mathrm{CDCl}_{3}\right)$
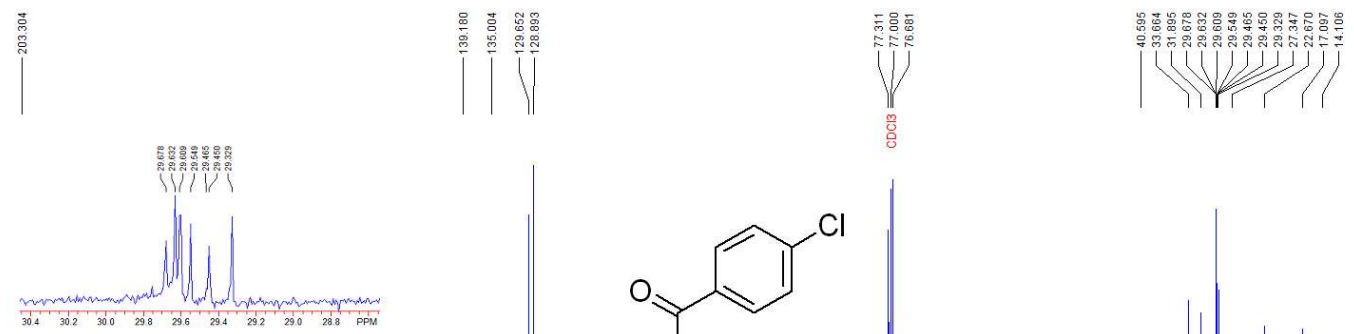

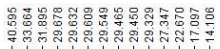

11
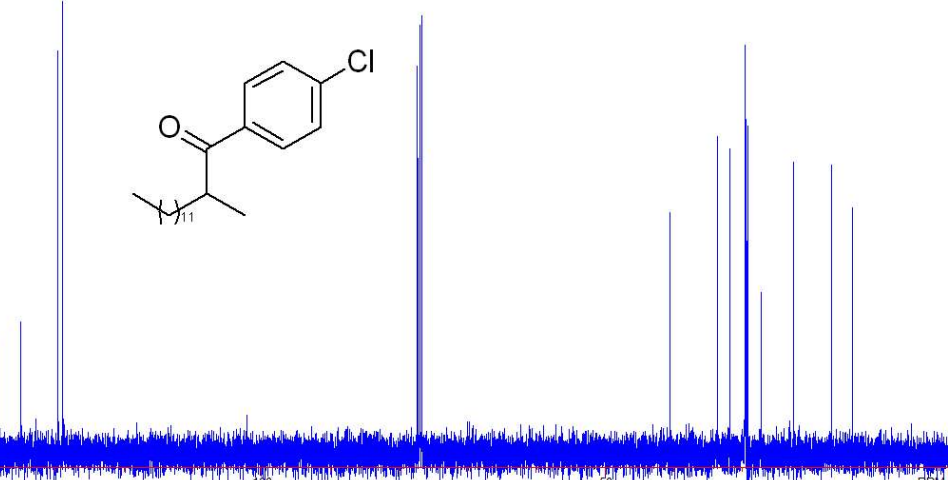


\section{Compound 4d}

${ }^{1} \mathrm{H}$ NMR (400 MHz, $\left.\mathrm{CDCl}_{3}\right)$

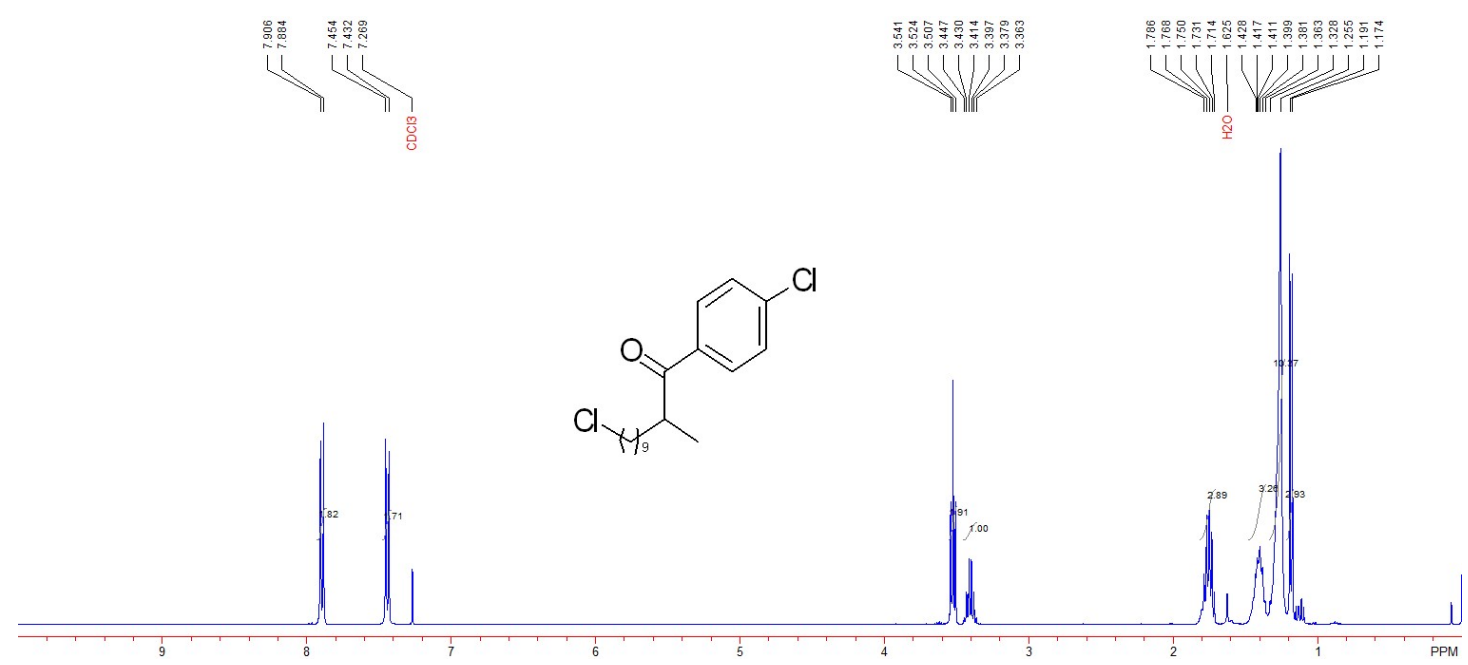

\section{Compound 4d}

${ }^{13} \mathrm{C} \mathrm{NMR}\left(101 \mathrm{MHz}, \mathrm{CDCl}_{3}\right)$

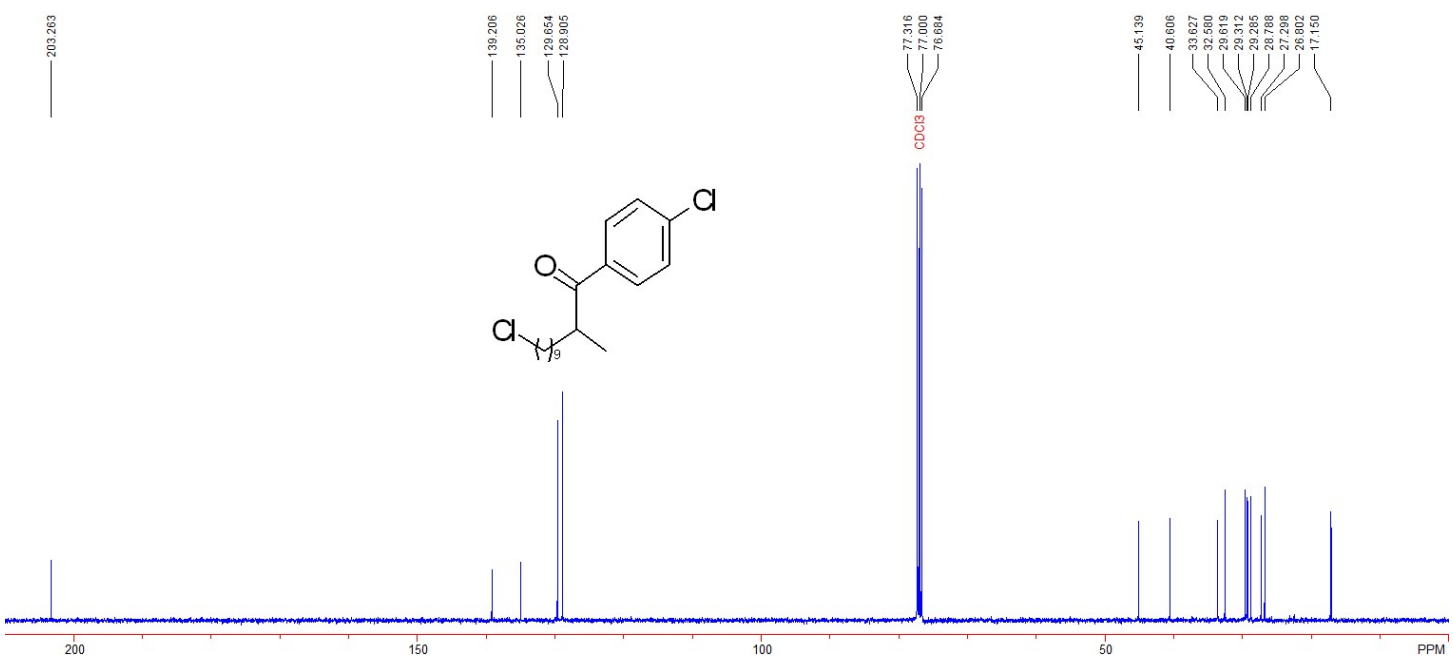




\section{Compound 4e}

${ }^{1} \mathrm{H}$ NMR (400 MHz, $\left.\mathrm{CDCl}_{3}\right)$

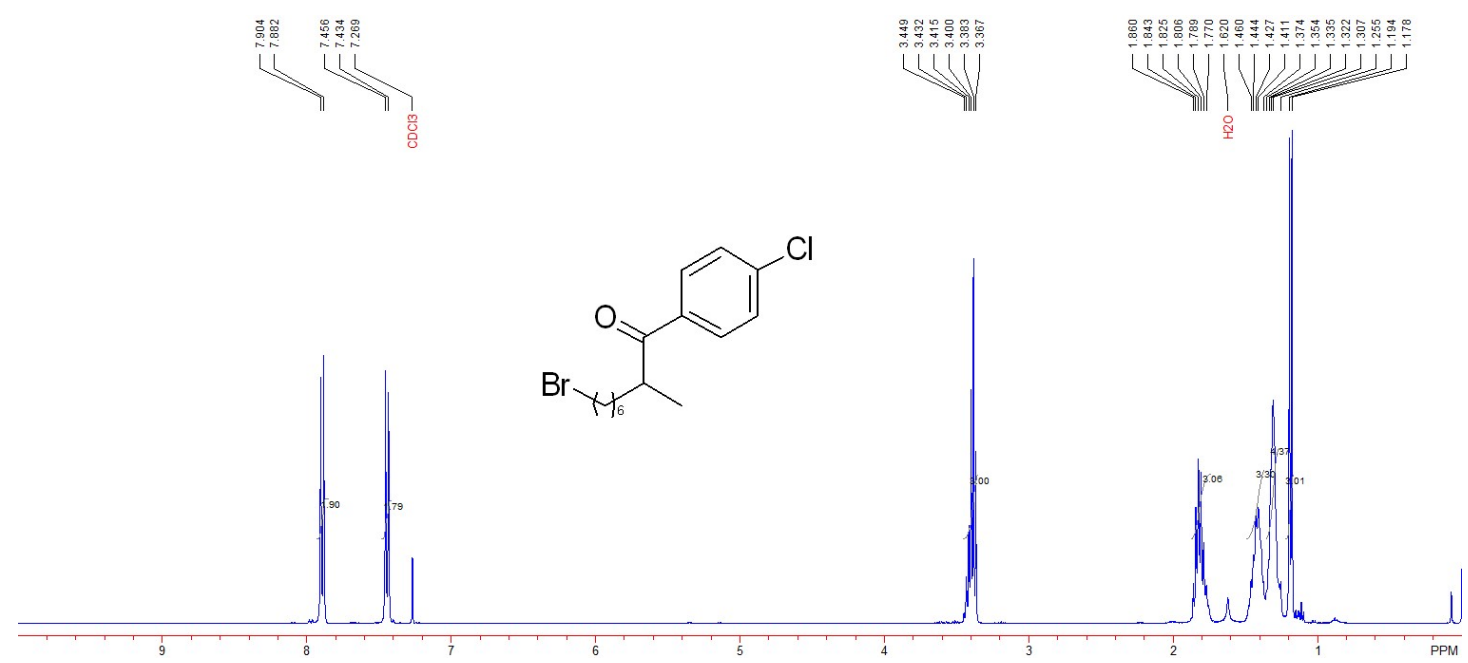

\section{Compound 4e}

${ }^{13} \mathrm{C} \mathrm{NMR}\left(101 \mathrm{MHz}, \mathrm{CDCl}_{3}\right)$
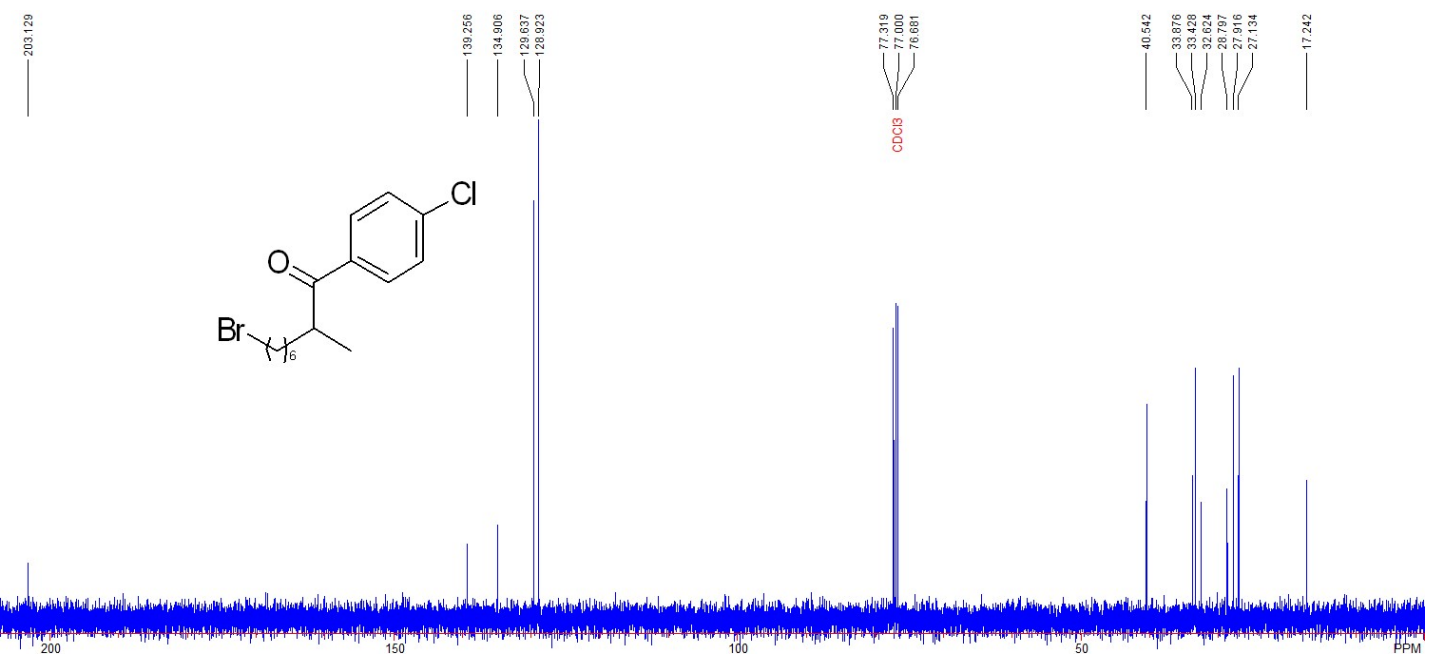


\section{Compound 4f}

${ }^{1} \mathrm{H}$ NMR (400 MHz, $\mathrm{CDCl}_{3}$ )

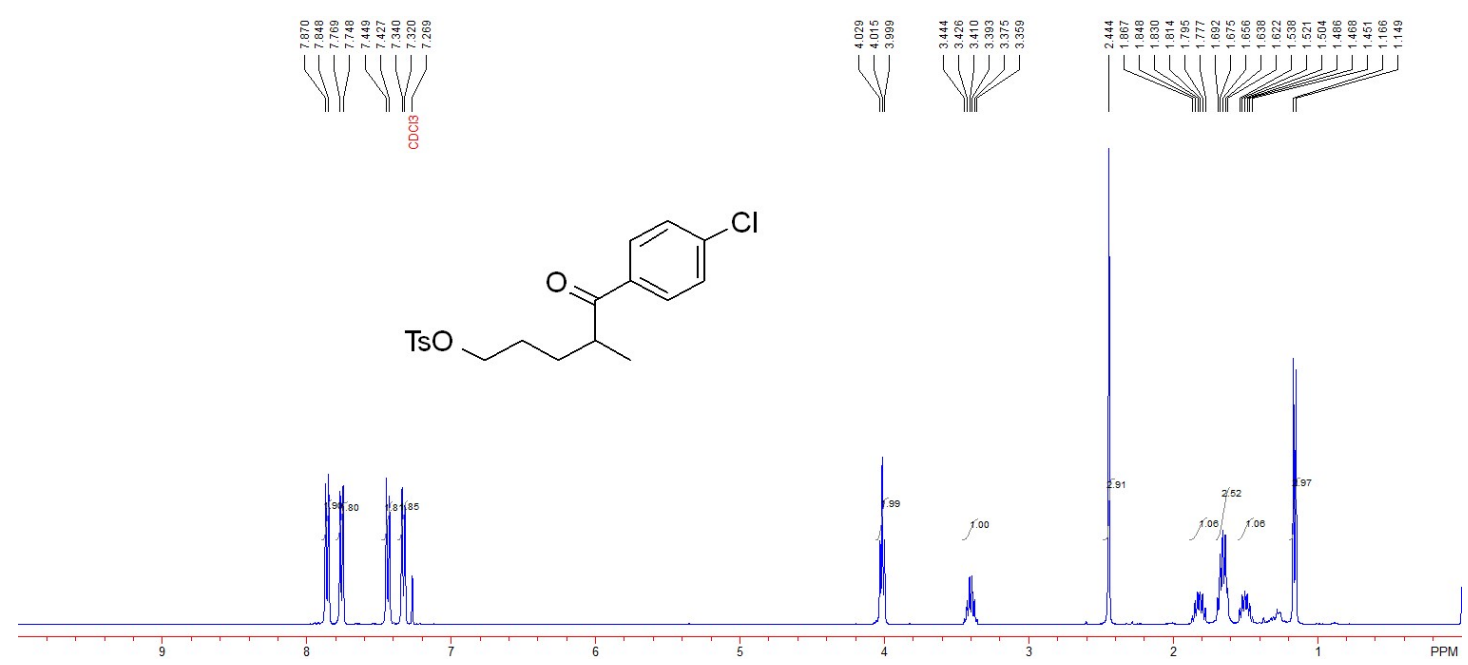

\section{Compound 4f}

${ }^{13} \mathrm{C} \mathrm{NMR}\left(101 \mathrm{MHz}, \mathrm{CDCl}_{3}\right)$
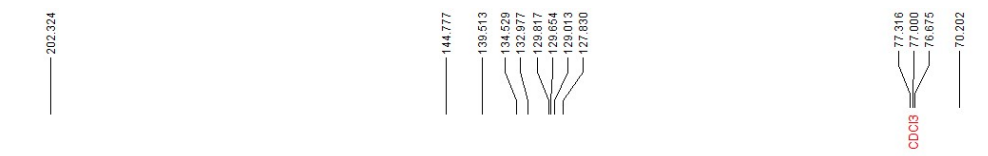

I lil|

$\mathrm{TsO}$
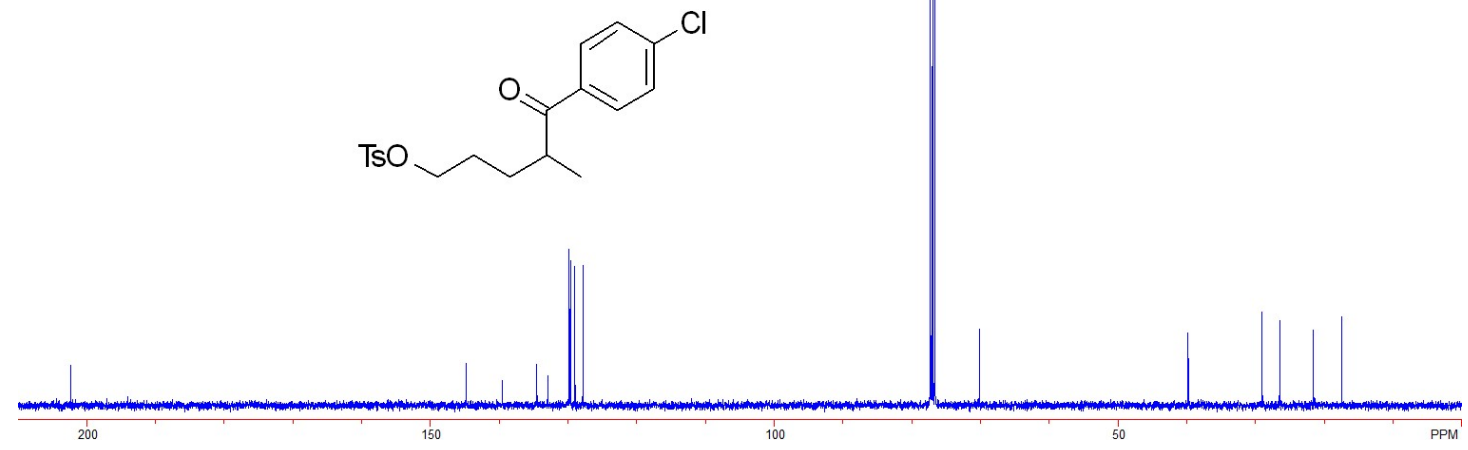


\section{Compound 4g}

${ }^{1} \mathrm{H}$ NMR (400 MHz, $\mathrm{CDCl}_{3}$ )

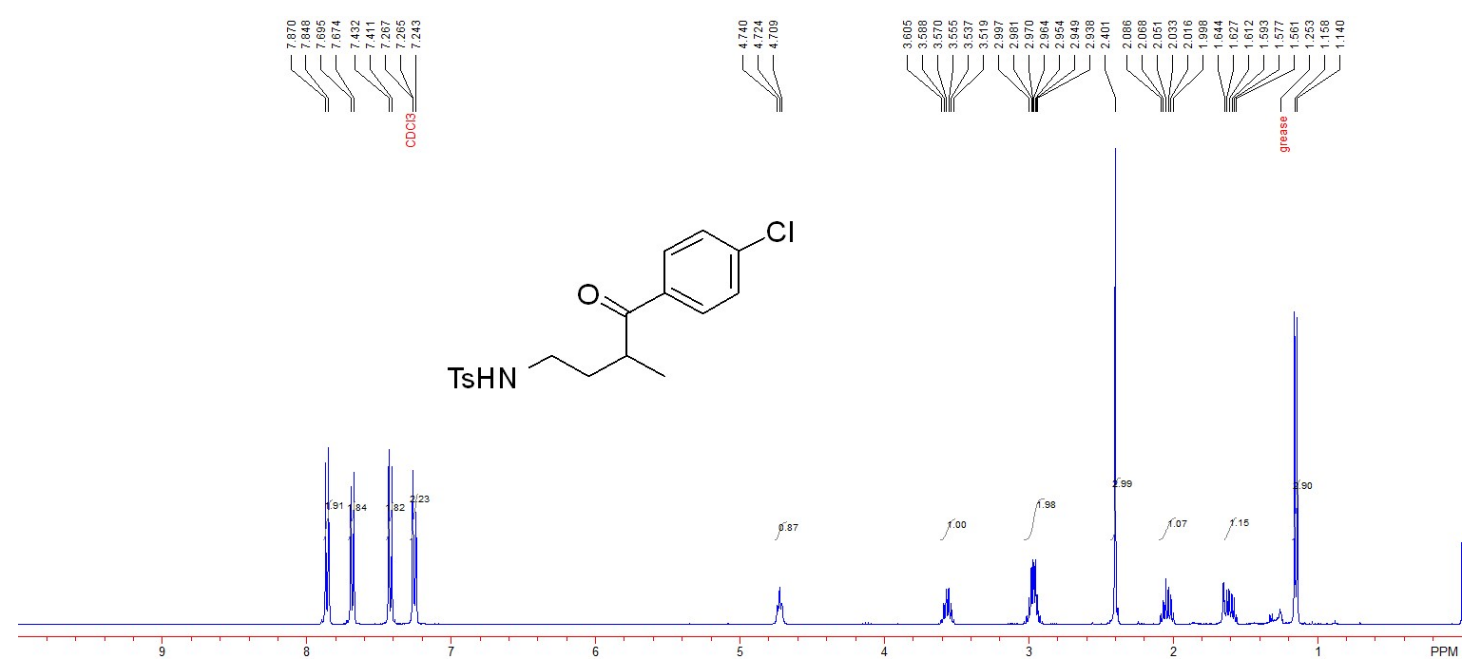

\section{Compound 4g}

${ }^{13} \mathrm{C} \mathrm{NMR}\left(101 \mathrm{MHz}, \mathrm{CDCl}_{3}\right)$
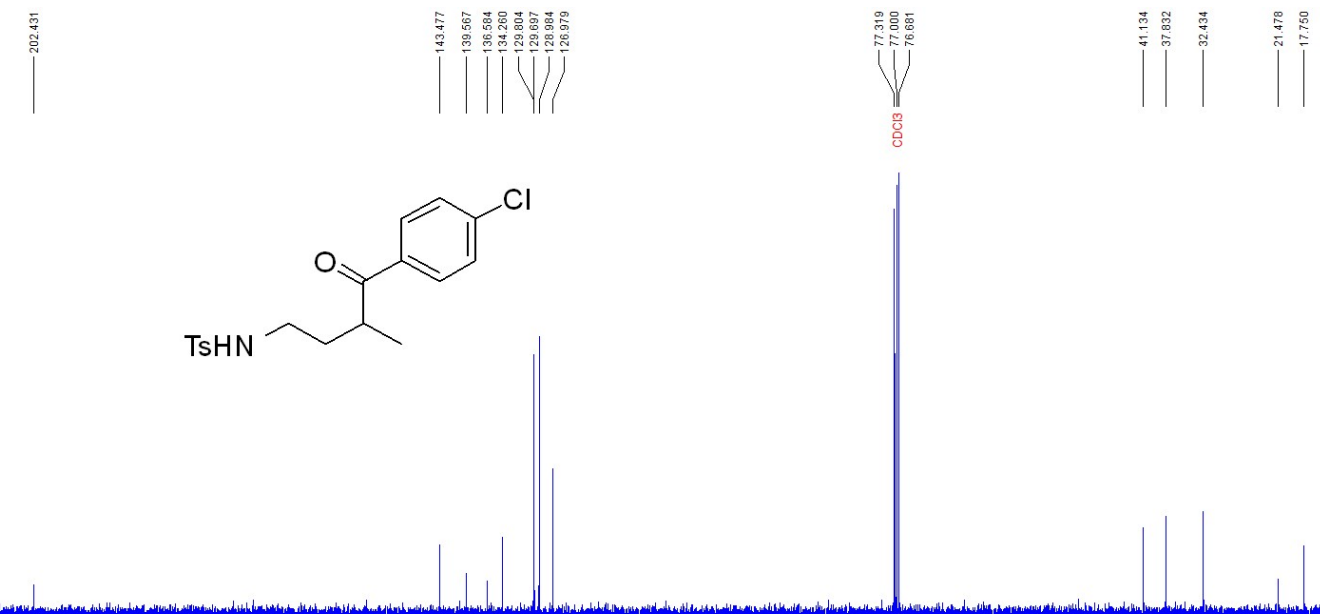


\section{Compound 4h}

${ }^{1} \mathrm{H} \mathrm{NMR}\left(400 \mathrm{MHz}, \mathrm{CDCl}_{3}\right)$

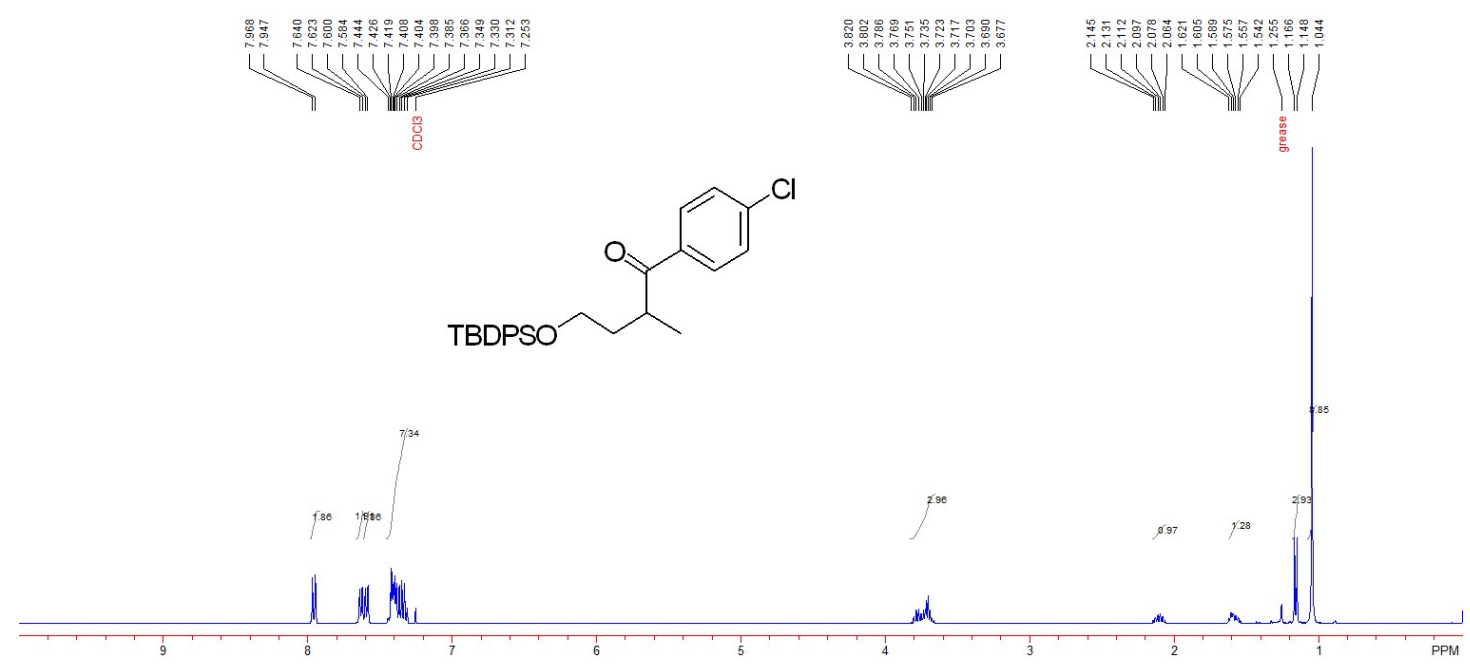

\section{Compound 4h}

${ }^{13} \mathrm{C} \mathrm{NMR}\left(101 \mathrm{MHz}, \mathrm{CDCl}_{3}\right)$

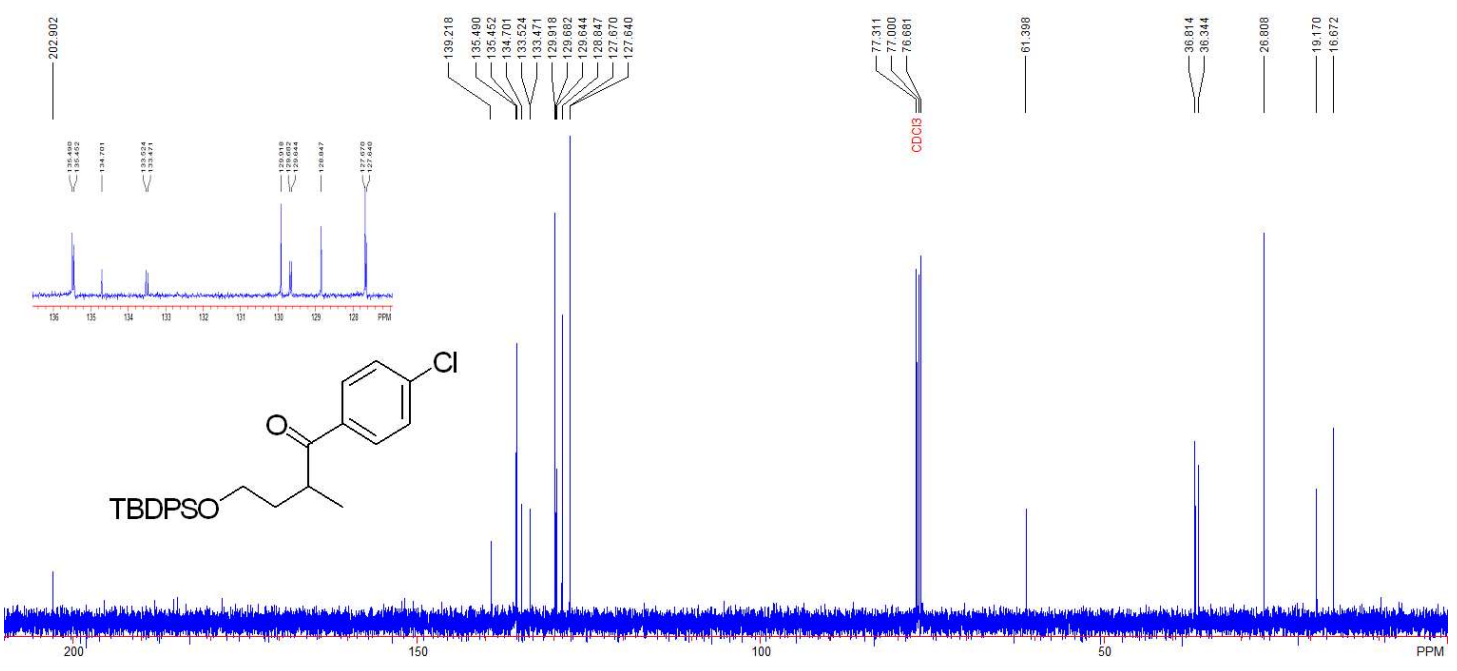




\section{Compound 4i}

${ }^{1} \mathrm{H}$ NMR (400 MHz, $\mathrm{CDCl}_{3}$ )

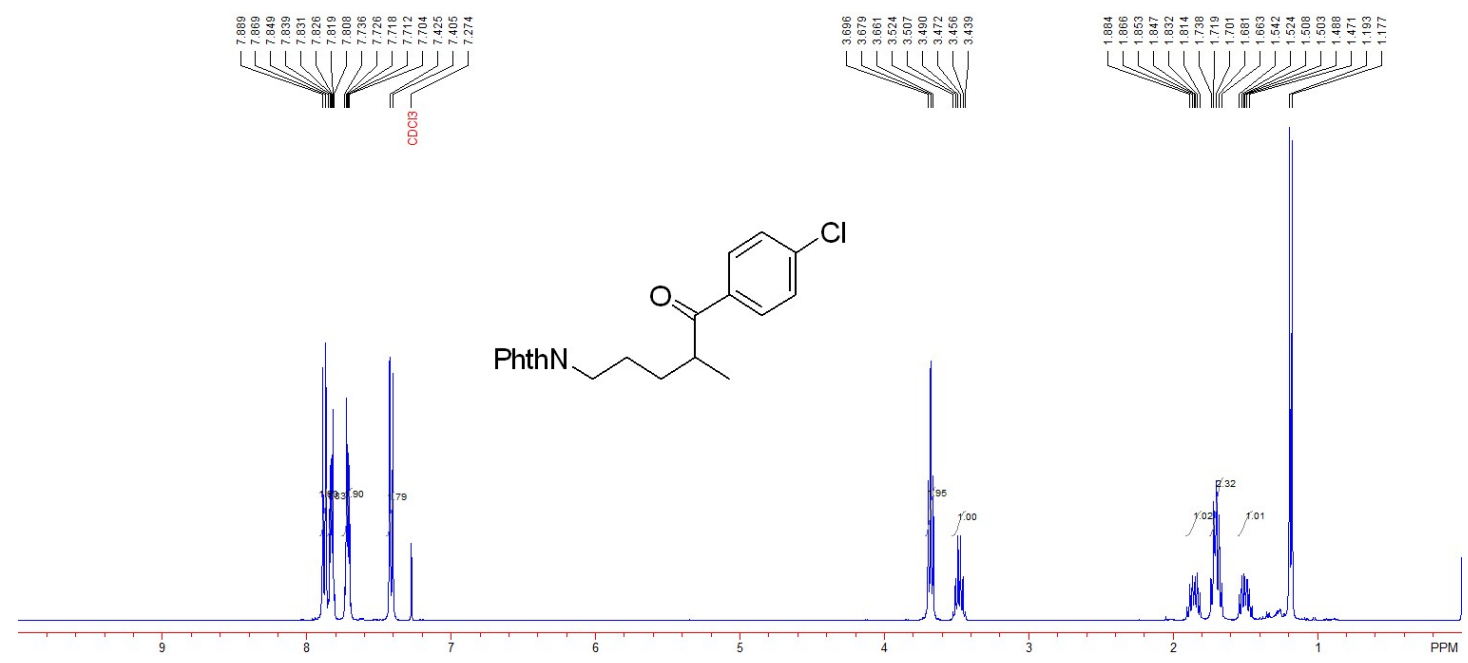

\section{Compound 4i}

${ }^{13} \mathrm{C} \mathrm{NMR}\left(101 \mathrm{MHz}, \mathrm{CDCl}_{3}\right)$
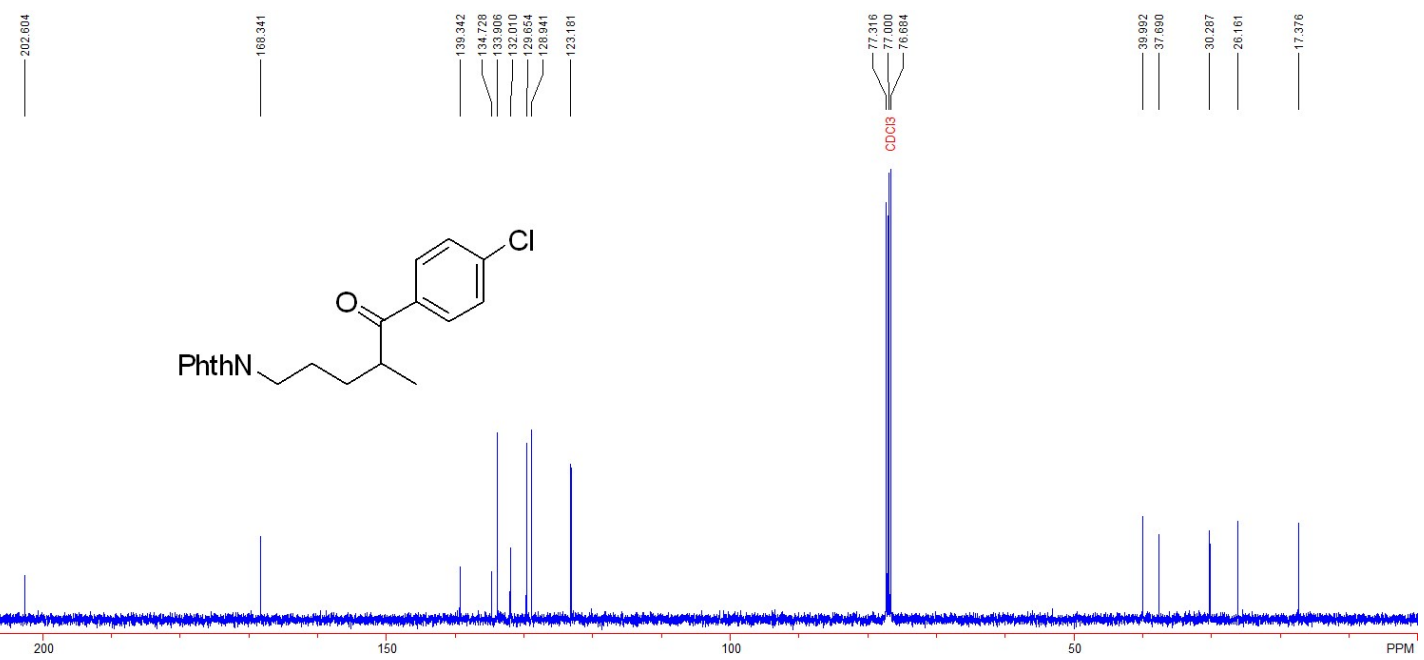


\section{Compound 4j}

${ }^{1} \mathrm{H}$ NMR (400 MHz, $\left.\mathrm{CDCl}_{3}\right)$

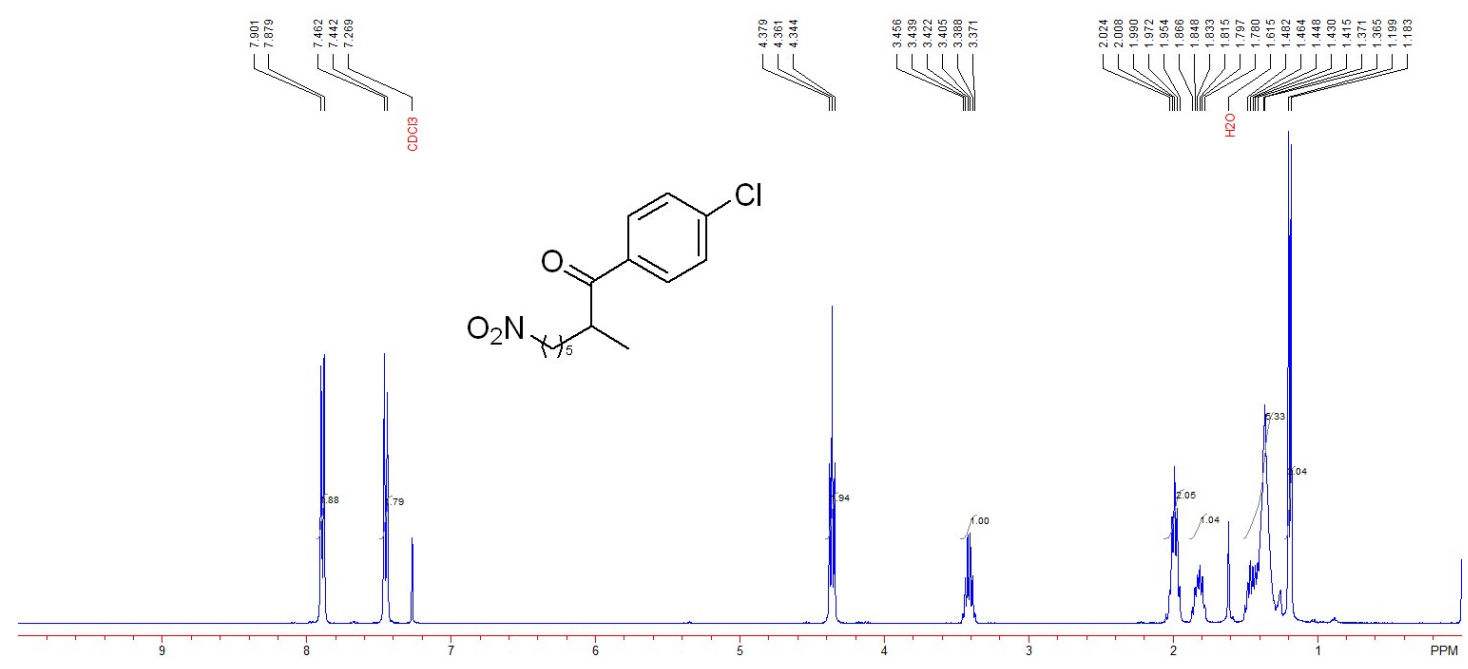

\section{Compound 4j}

${ }^{13} \mathrm{C} \mathrm{NMR}\left(101 \mathrm{MHz}, \mathrm{CDCl}_{3}\right)$
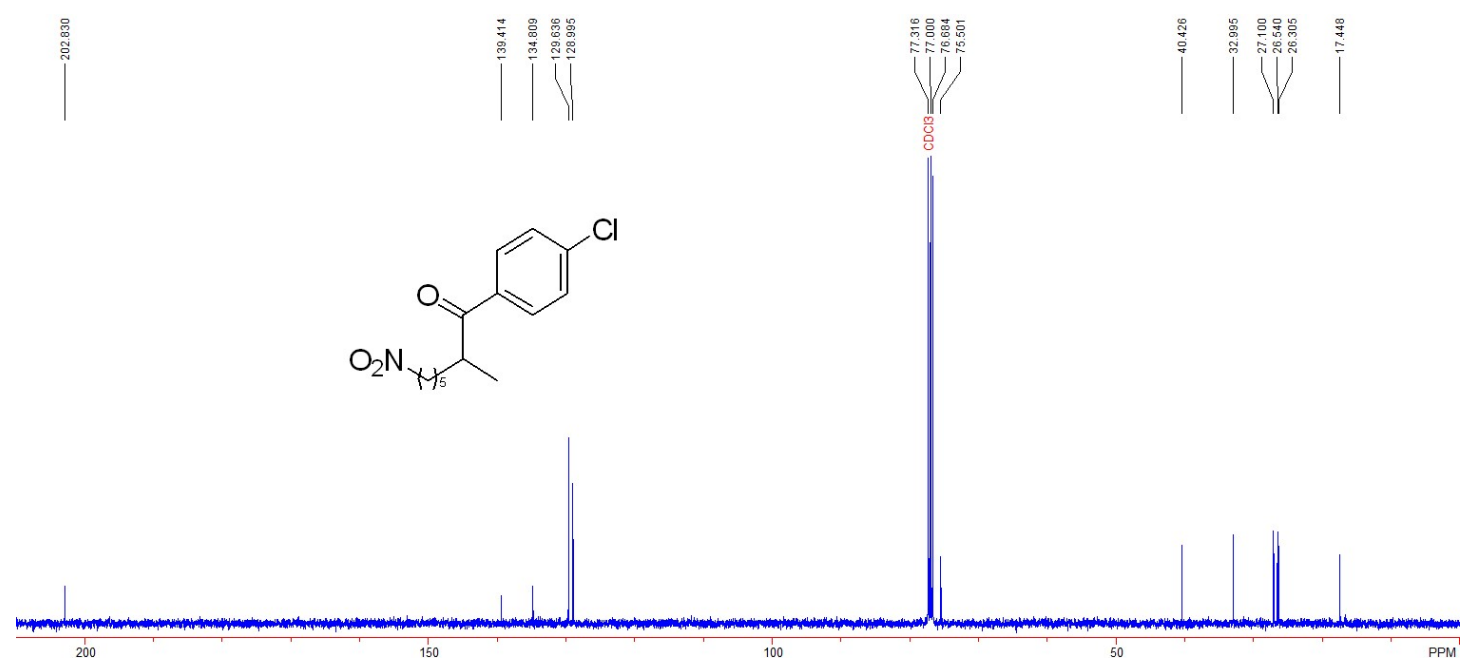


\section{Compound 4k}

${ }^{1} \mathrm{H}$ NMR (400 MHz, $\mathrm{CDCl}_{3}$ )
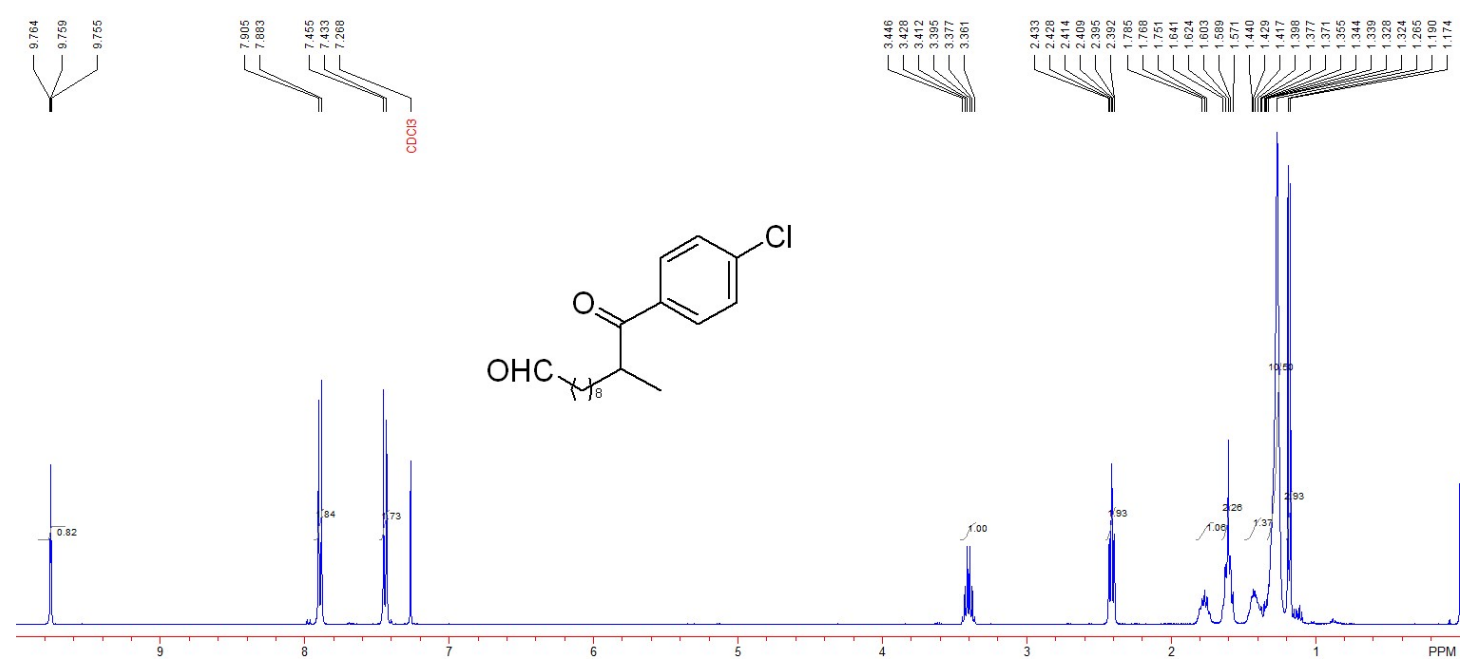

\section{Compound 4k}

${ }^{13} \mathrm{C} \mathrm{NMR}\left(101 \mathrm{MHz}, \mathrm{CDCl}_{3}\right)$

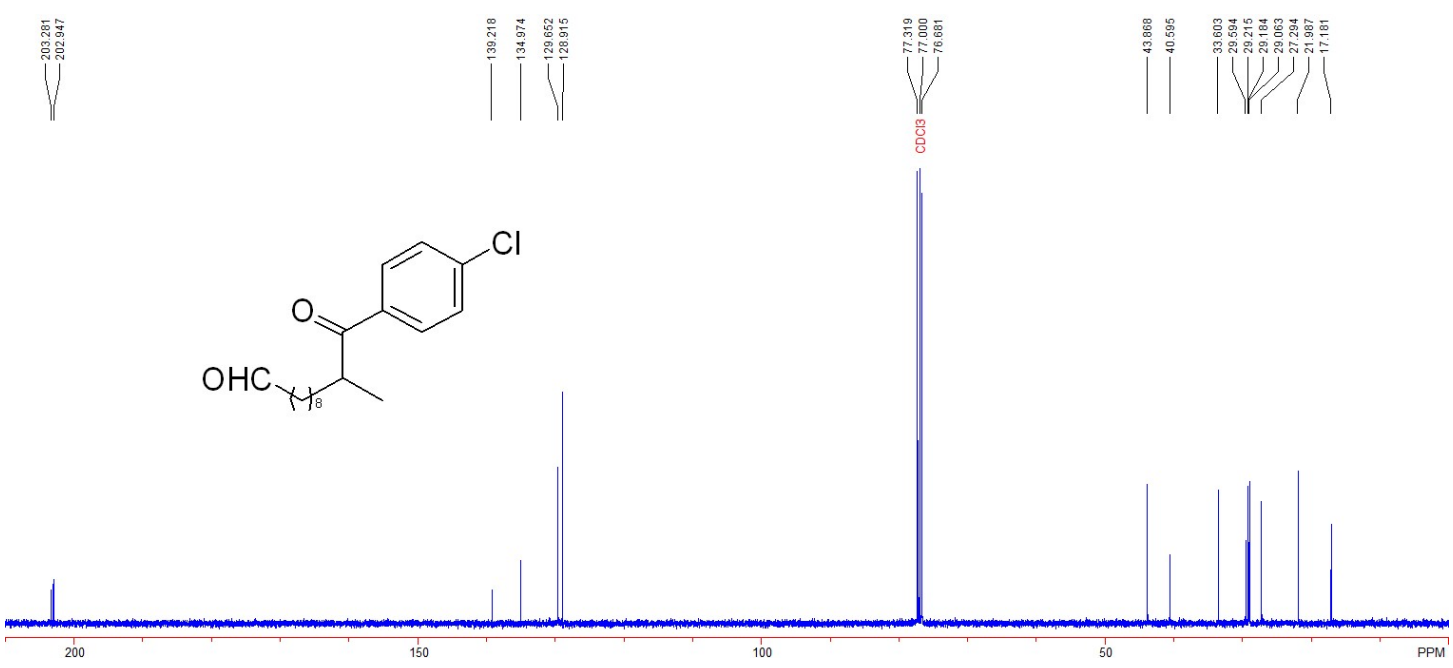




\section{Compound 41}

${ }^{1} \mathrm{H}$ NMR (400 MHz, $\mathrm{CDCl}_{3}$ )
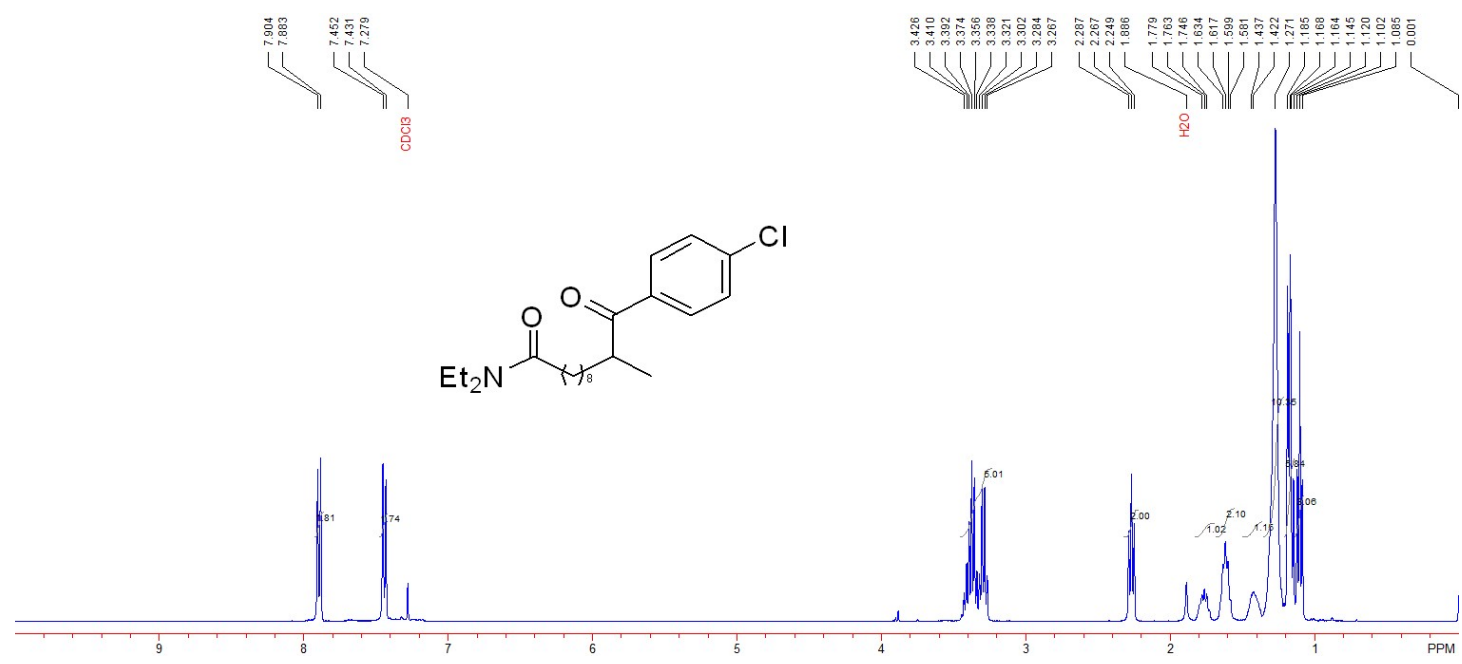

\section{Compound 41}

${ }^{13} \mathrm{C} \mathrm{NMR}\left(101 \mathrm{MHz}, \mathrm{CDCl}_{3}\right)$
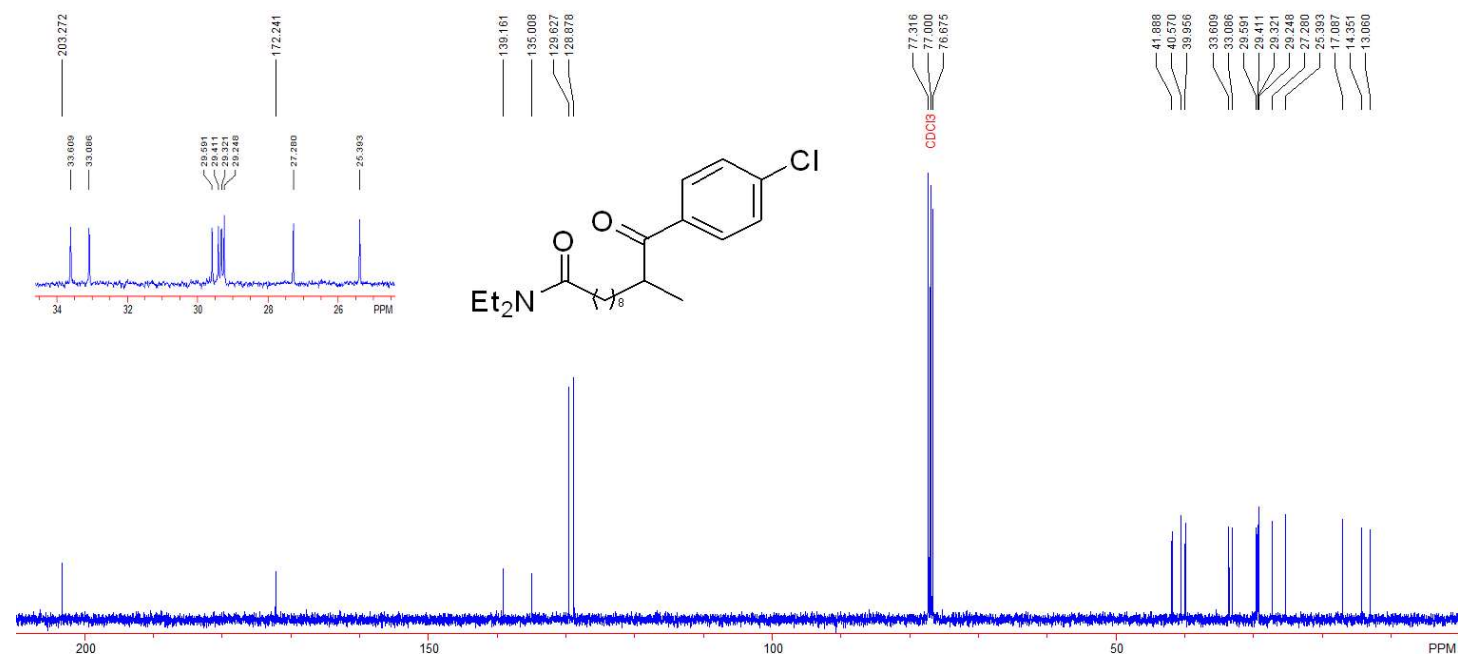


\section{Compound 4m}

${ }^{1} \mathrm{H}$ NMR (400 MHz, $\mathrm{CDCl}_{3}$ )
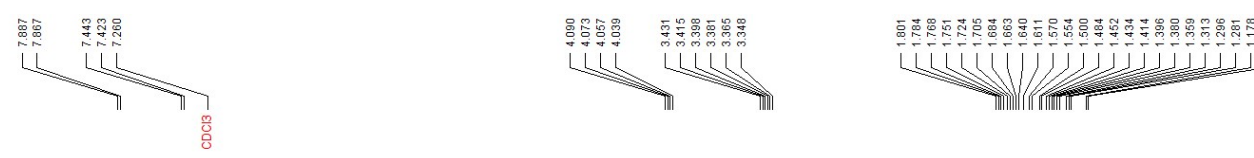

LLUUVIIIJIJIIJ

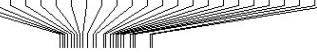<smiles>CCOP(=O)(NC(C)C(=O)c1ccc(Cl)cc1)OCC</smiles>

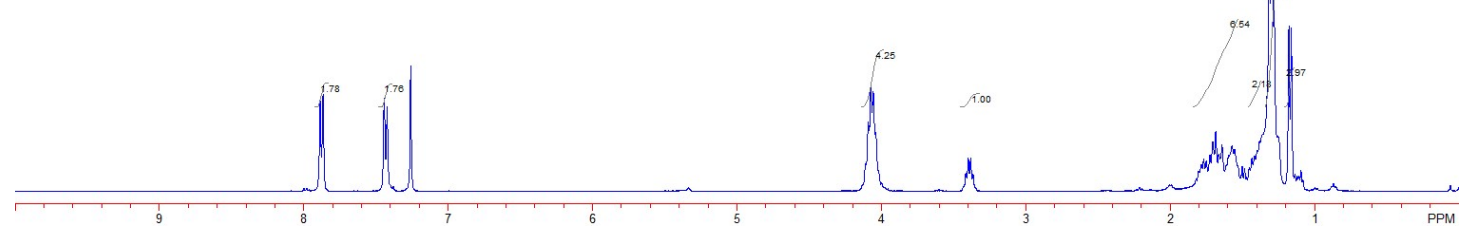

\section{Compound 4m}

${ }^{13} \mathrm{C} \mathrm{NMR}\left(101 \mathrm{MHz}, \mathrm{CDCl}_{3}\right)$
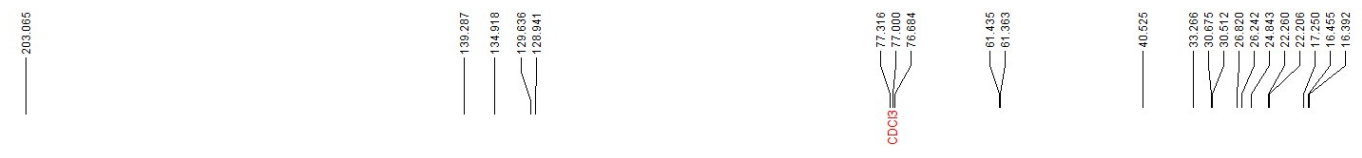<smiles>CCOP(=O)(NC(C)C(=O)c1ccc(Cl)cc1)OCC</smiles>

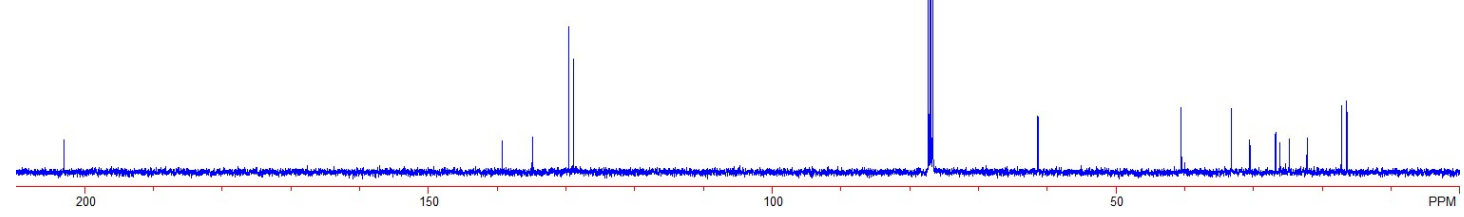


Compound 4n ${ }^{1} \mathrm{H}$ NMR (400 MHz, $\left.\mathrm{CDCl}_{3}\right)$
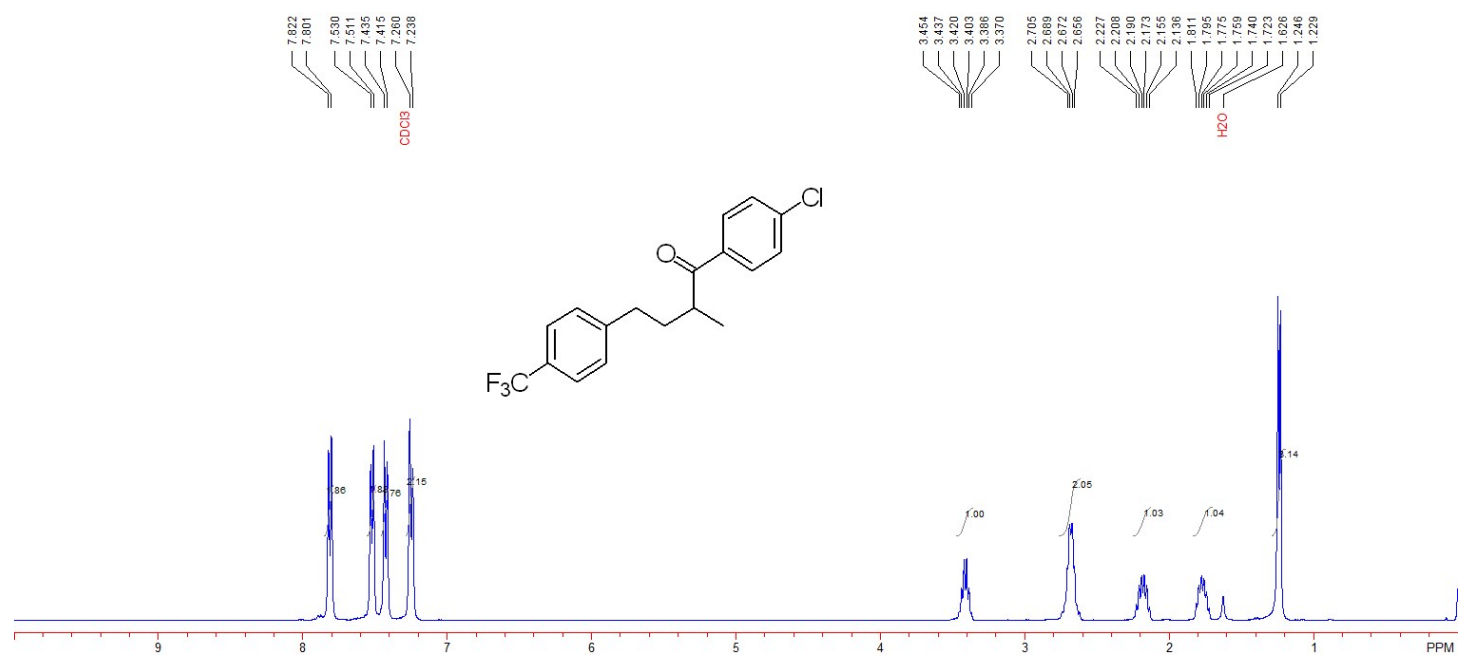

Compound 4n ${ }^{19} \mathrm{~F}$ NMR (376 MHz, $\left.\mathrm{CDCl}_{3}\right)$

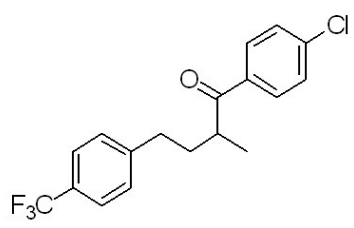

Compound $4 \mathbf{n}{ }^{13} \mathrm{C}$ NMR (101 MHz, $\left.\mathrm{CDCl}_{3}\right)$

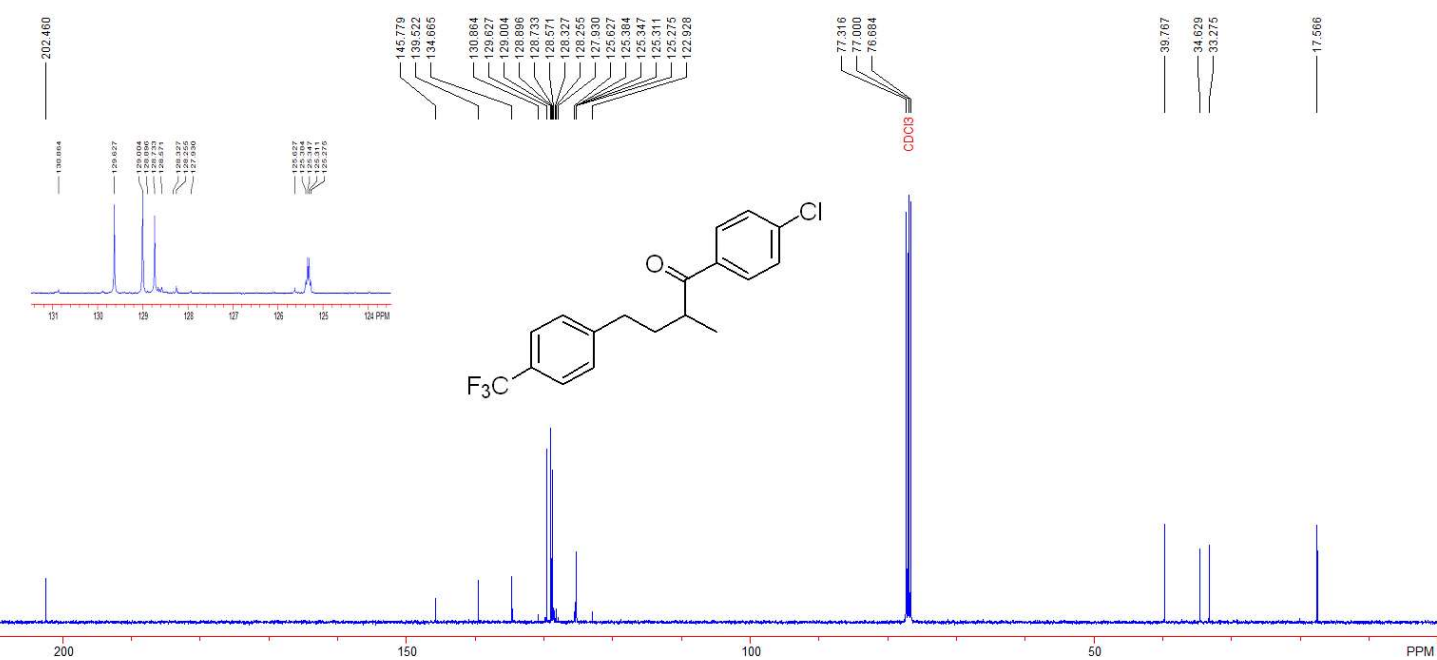




\section{Compound 4o}

${ }^{1} \mathrm{H}$ NMR (400 MHz, $\left.\mathrm{CDCl}_{3}\right)$

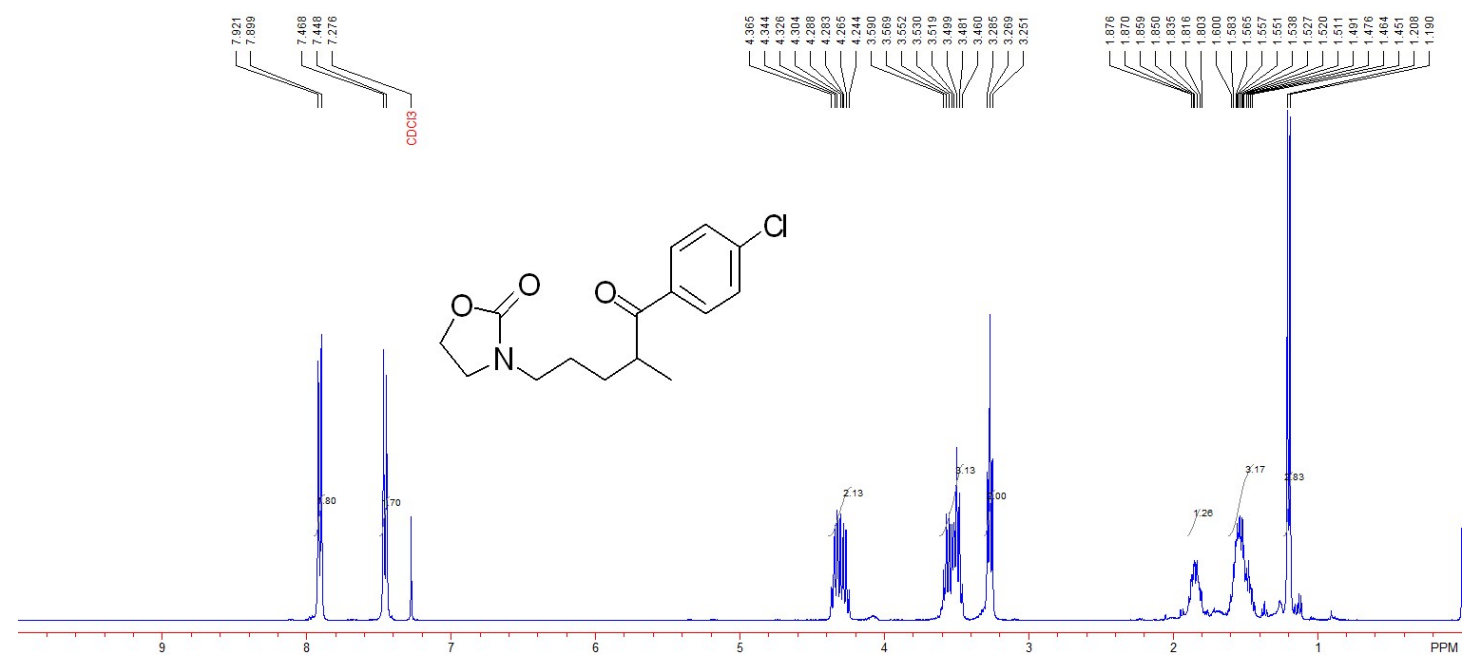

\section{Compound 4o}

${ }^{13} \mathrm{C} \mathrm{NMR}\left(101 \mathrm{MHz}, \mathrm{CDCl}_{3}\right)$

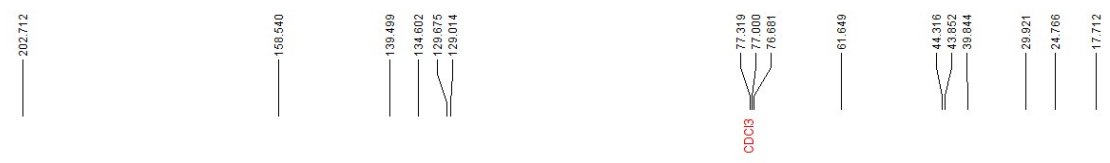<smiles>CC(CCCN1CCOC1=O)C(=O)c1ccc(Cl)cc1</smiles> 


\section{Compound 4p}

${ }^{1} \mathrm{H}$ NMR (400 MHz, $\mathrm{CDCl}_{3}$ )

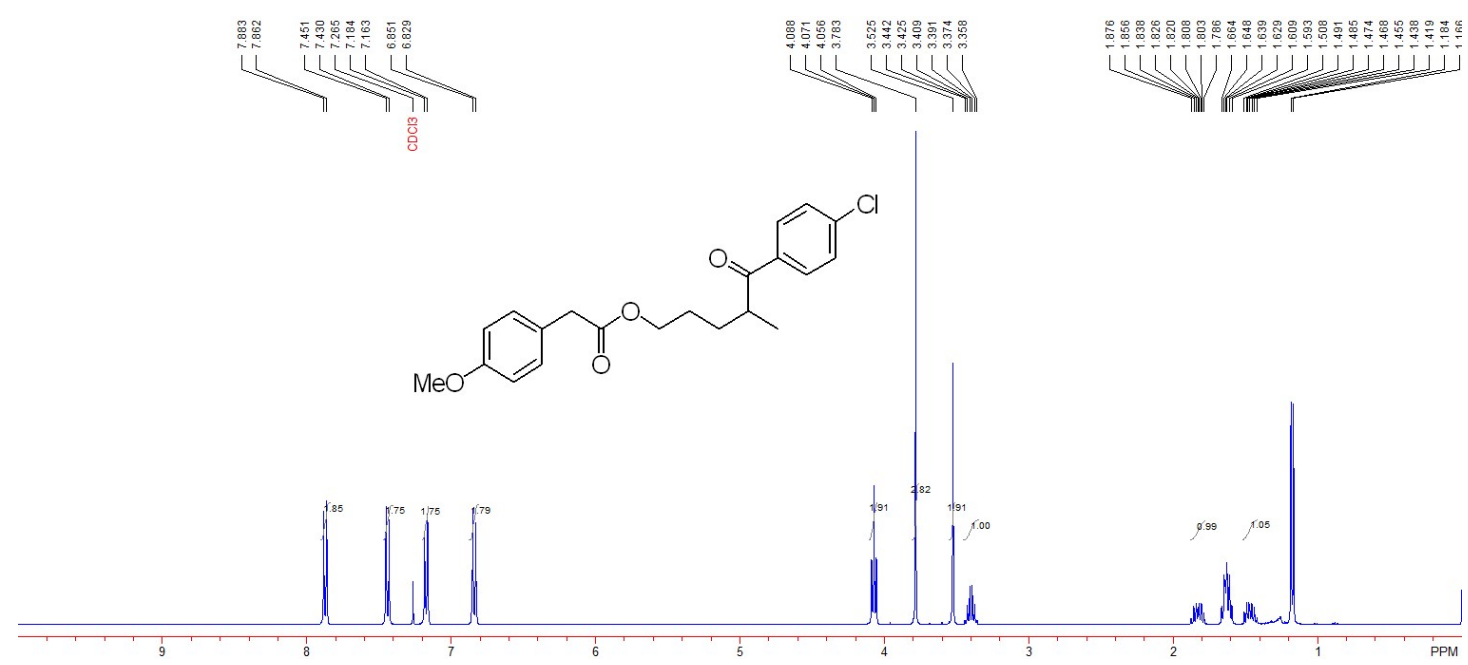

\section{Compound 4p}

${ }^{13} \mathrm{C} \mathrm{NMR}\left(101 \mathrm{MHz}, \mathrm{CDCl}_{3}\right)$
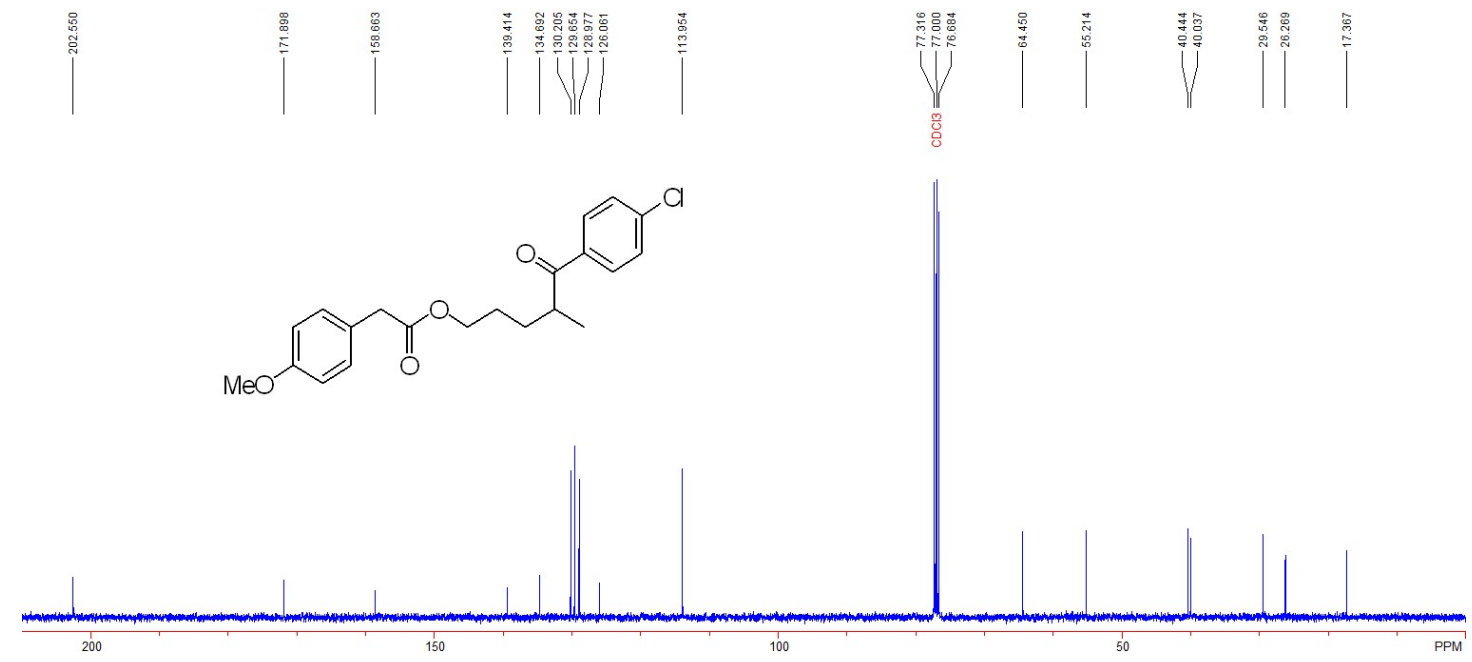


\section{Compound 4q}

${ }^{1} \mathrm{H}$ NMR (400 MHz, $\left.\mathrm{CDCl}_{3}\right)$

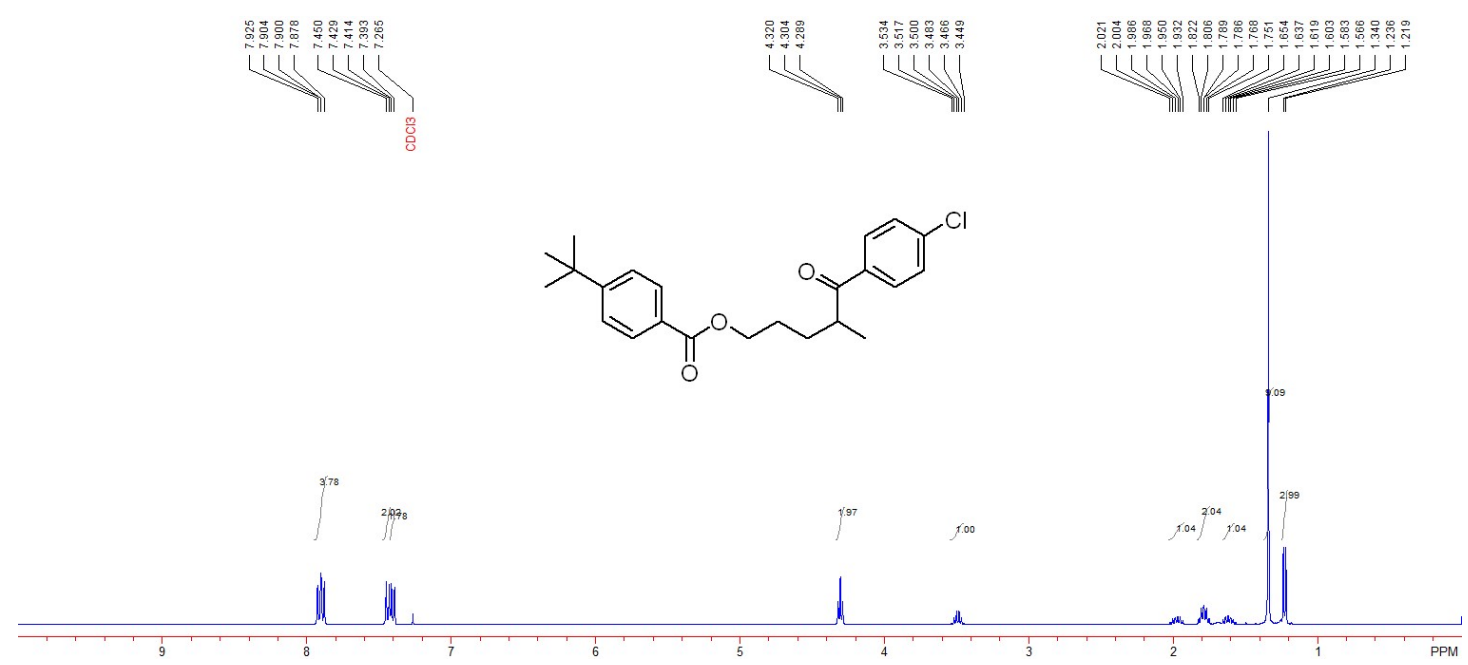

\section{Compound 4q}

${ }^{13} \mathrm{C} \mathrm{NMR}\left(101 \mathrm{MHz}, \mathrm{CDCl}_{3}\right)$
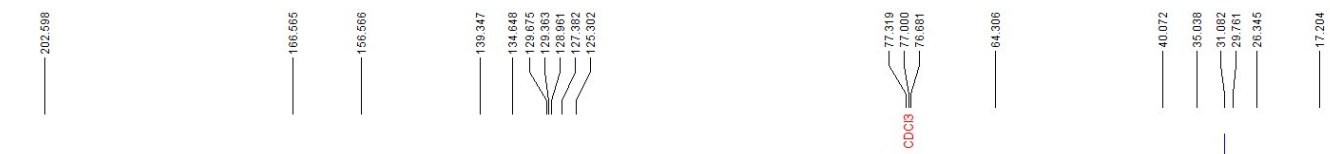<smiles>CC(CCCOC(=O)c1ccc(C(C)(C)C)cc1)C(=O)c1ccc(Cl)cc1</smiles>

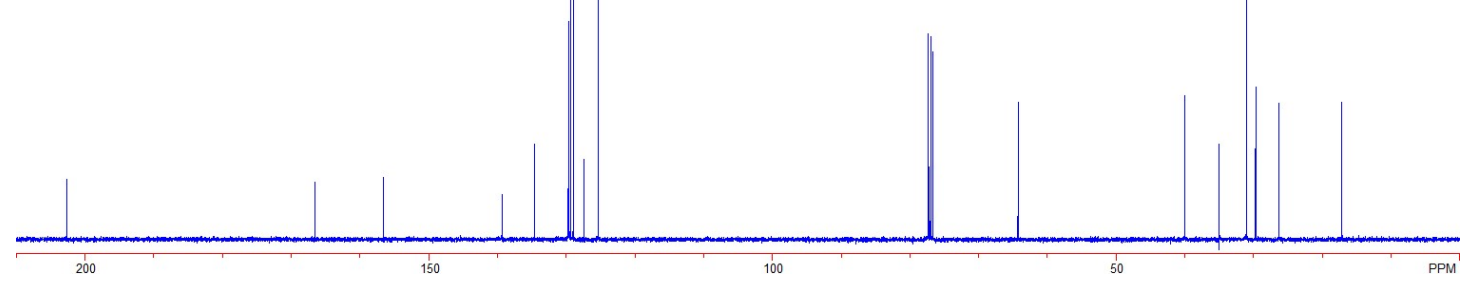




\section{Compound 4r}

${ }^{1} \mathrm{H}$ NMR (400 MHz, $\mathrm{CDCl}_{3}$ )

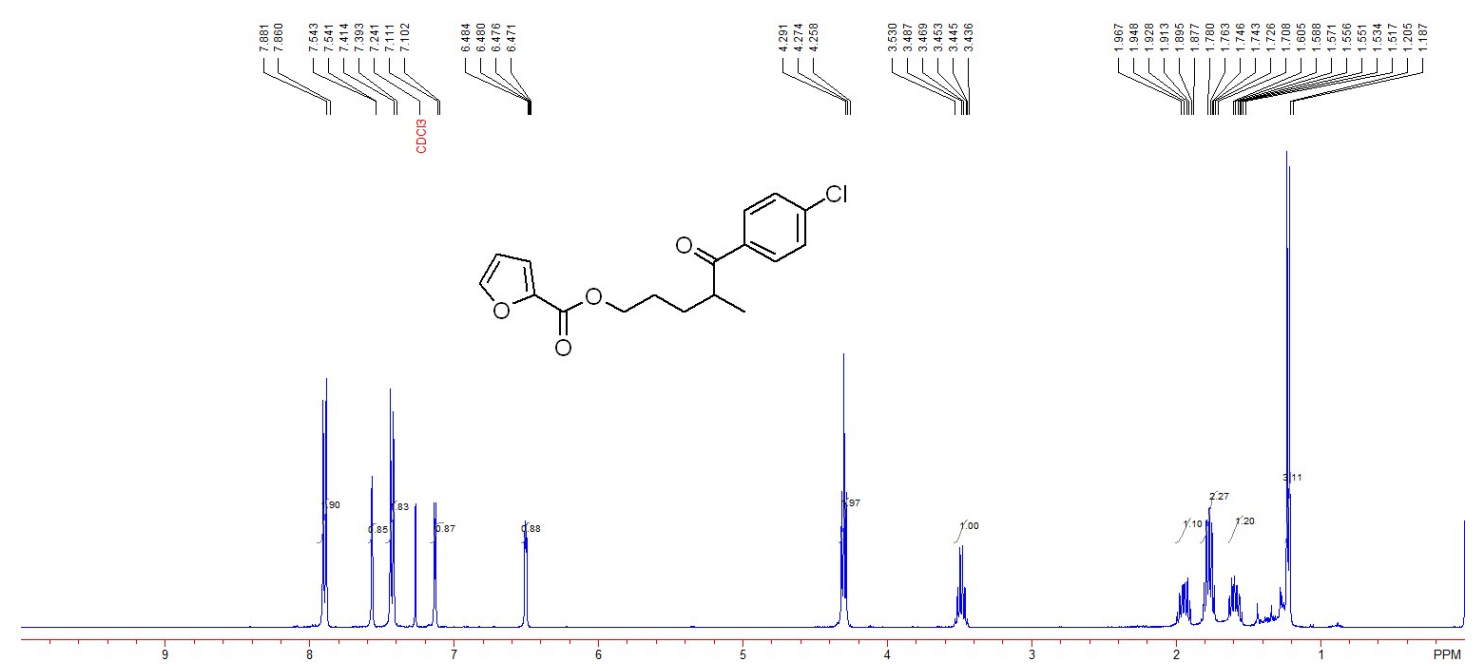

\section{Compound 4r}

${ }^{13} \mathrm{C} \mathrm{NMR}\left(101 \mathrm{MHz}, \mathrm{CDCl}_{3}\right)$

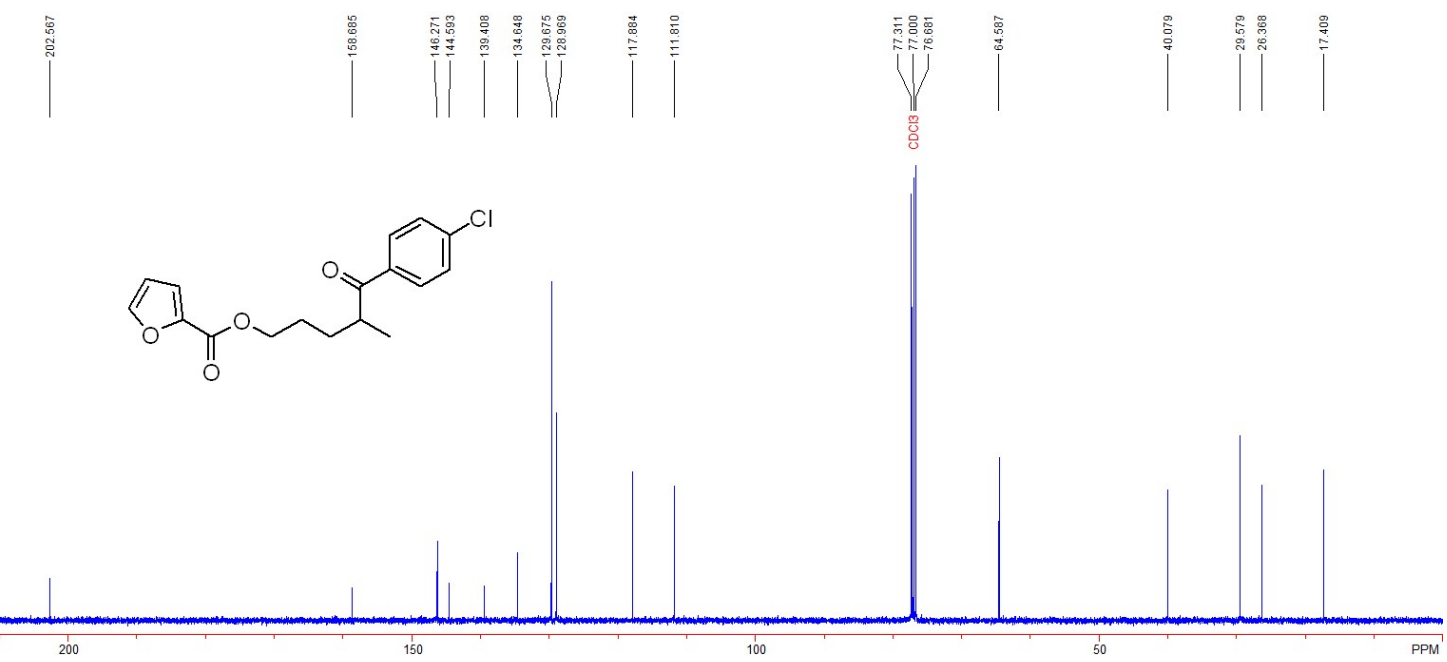




\section{Compound 4s}

${ }^{1} \mathrm{H}$ NMR (400 MHz, $\mathrm{CDCl}_{3}$ )

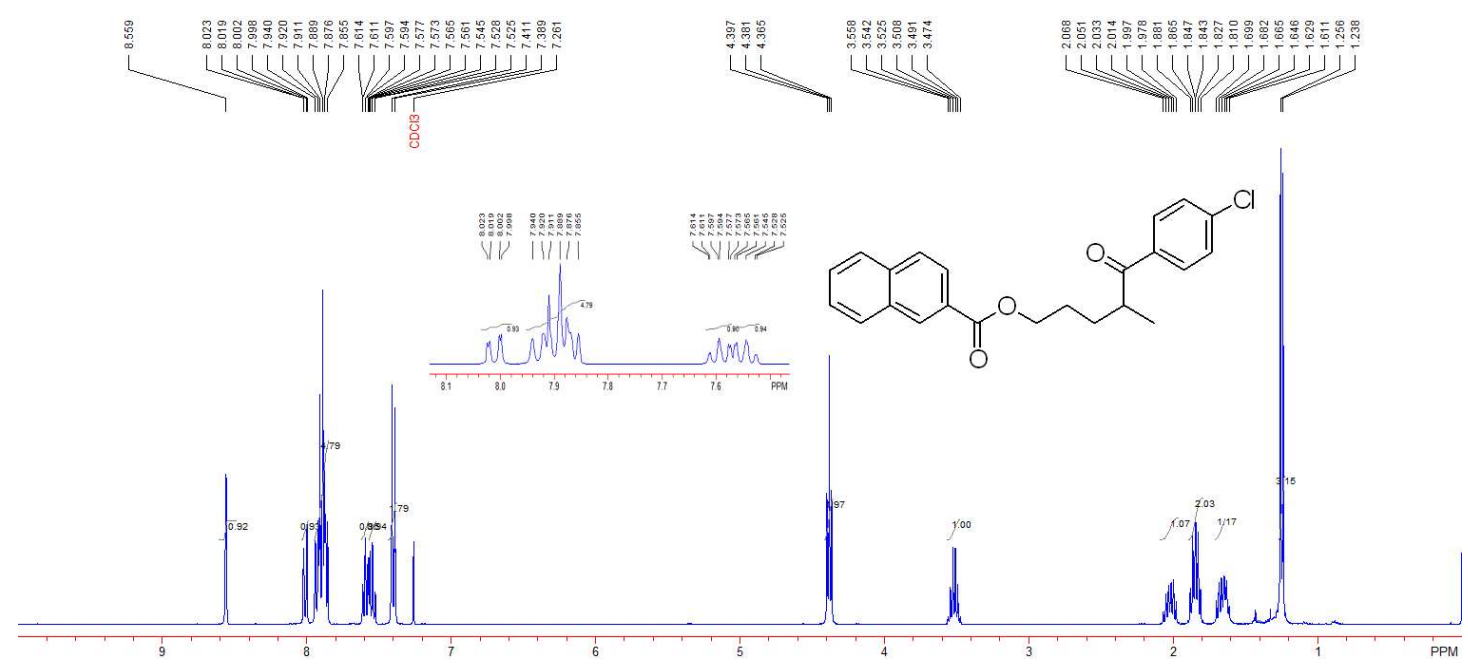

\section{Compound 4s}

${ }^{13} \mathrm{C} \mathrm{NMR}\left(101 \mathrm{MHz}, \mathrm{CDCl}_{3}\right)$
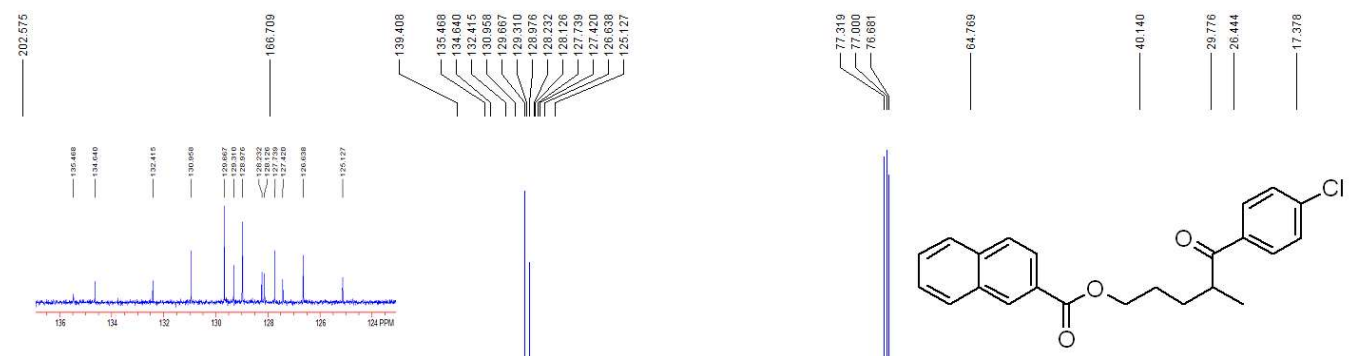


\section{Compound 4t}

${ }^{1} \mathrm{H}$ NMR (400 MHz, $\left.\mathrm{CDCl}_{3}\right)$

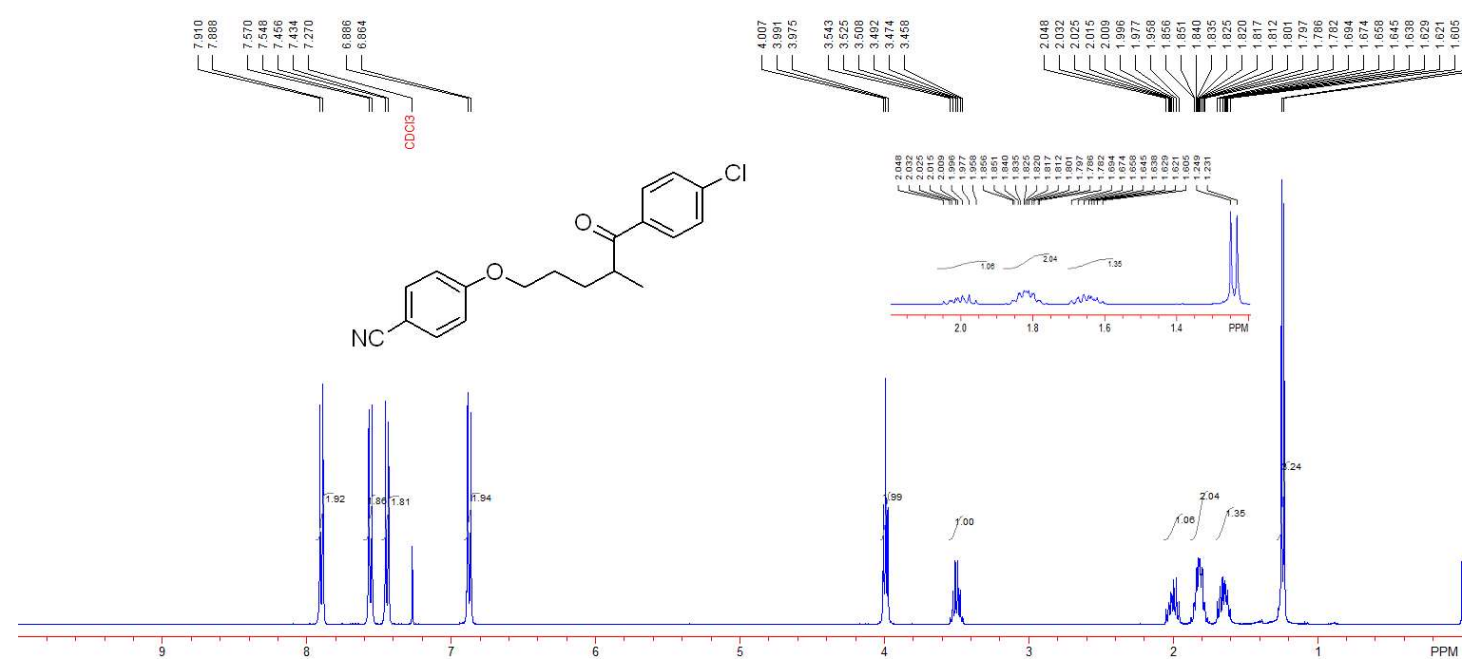

\section{Compound 4t}

${ }^{13} \mathrm{C} \mathrm{NMR}\left(101 \mathrm{MHz}, \mathrm{CDCl}_{3}\right)$

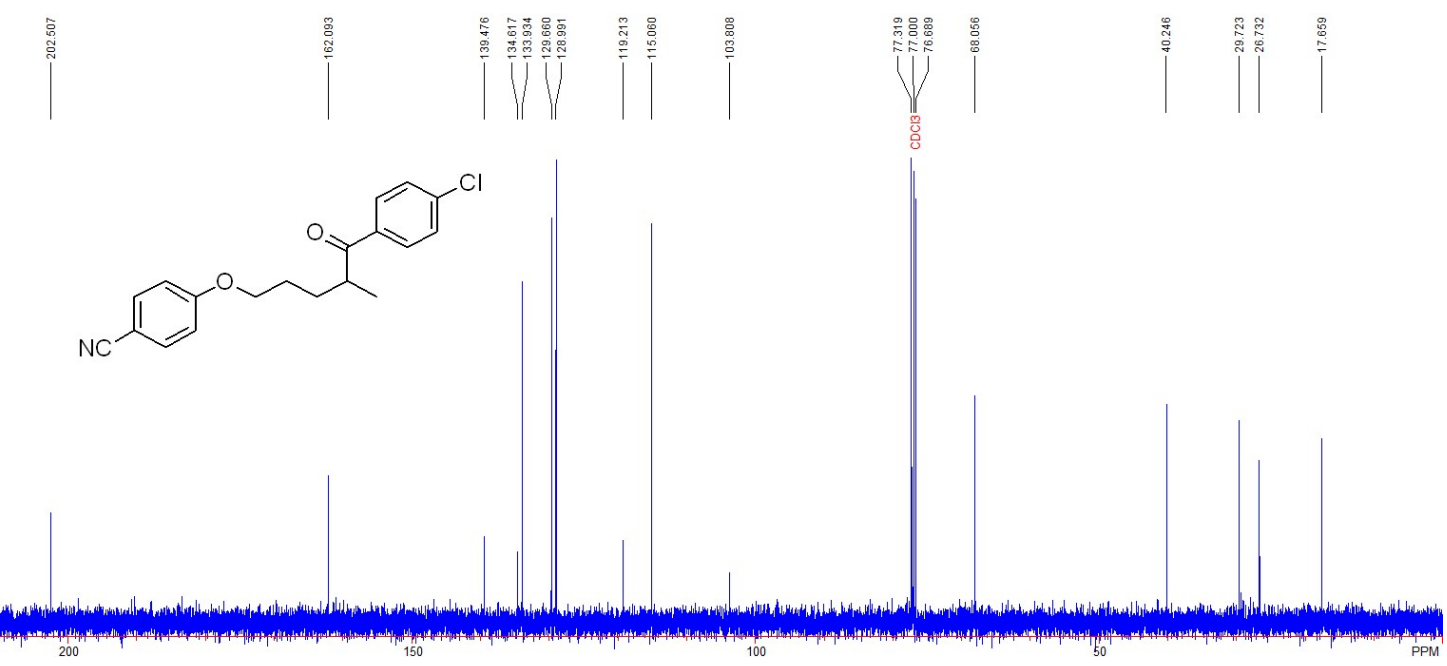




\section{Compound 4u}

${ }^{1} \mathrm{H}$ NMR (400 MHz, $\mathrm{CDCl}_{3}$ )
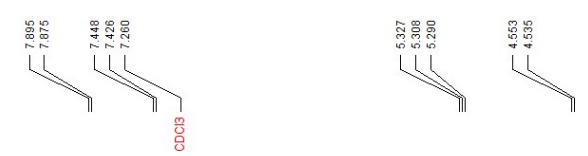

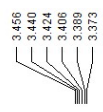

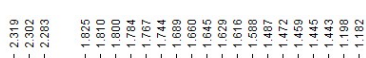

uiviviJisisis

Th<smiles>CC(C)=CCOC(=O)CCCC(C)C(=O)c1ccc(Cl)cc1</smiles>

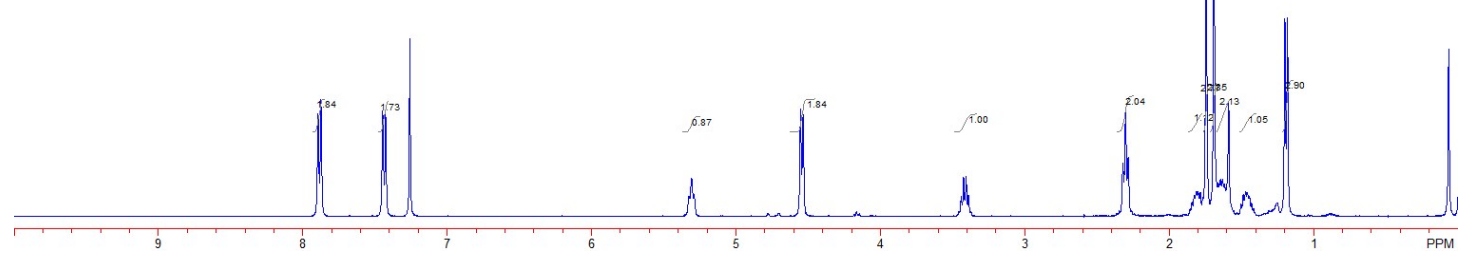

\section{Compound $4 u$}

${ }^{13} \mathrm{C} \mathrm{NMR}\left(101 \mathrm{MHz}, \mathrm{CDCl}_{3}\right)$

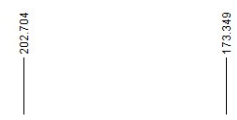<smiles>CC(C)=CCOC(=O)CCCC(C)C(=O)c1ccc(Cl)cc1</smiles>

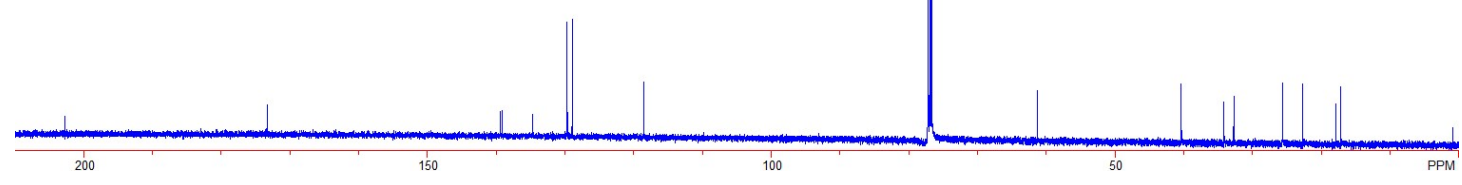


Compound Av

${ }^{1} \mathrm{H}$ NMR (400 MHz, $\mathrm{CDCl}_{3}$ )
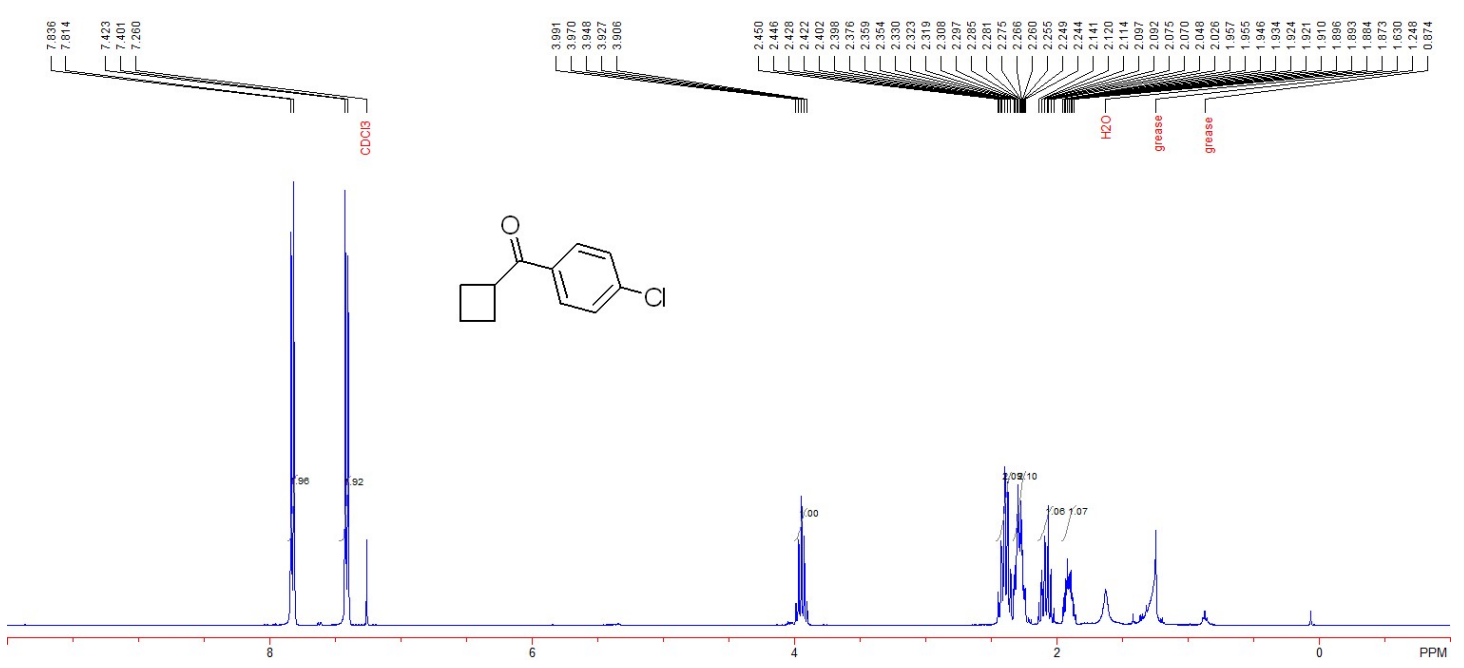

Compound Av

${ }^{13} \mathrm{C}$ NMR (101 MHz, $\mathrm{CDCl}_{3}$ )

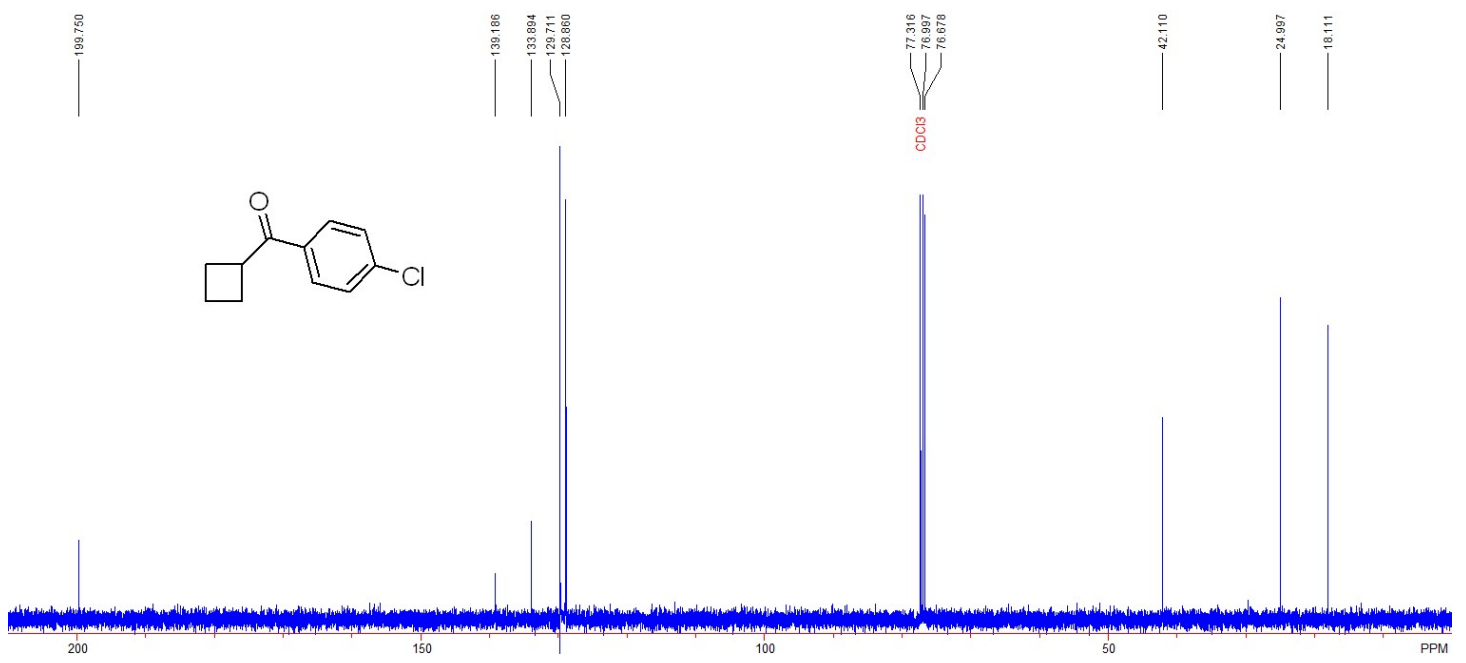

S95 


\section{Compound 4w}

${ }^{1} \mathrm{H}$ NMR (400 MHz, $\mathrm{CDCl}_{3}$ )

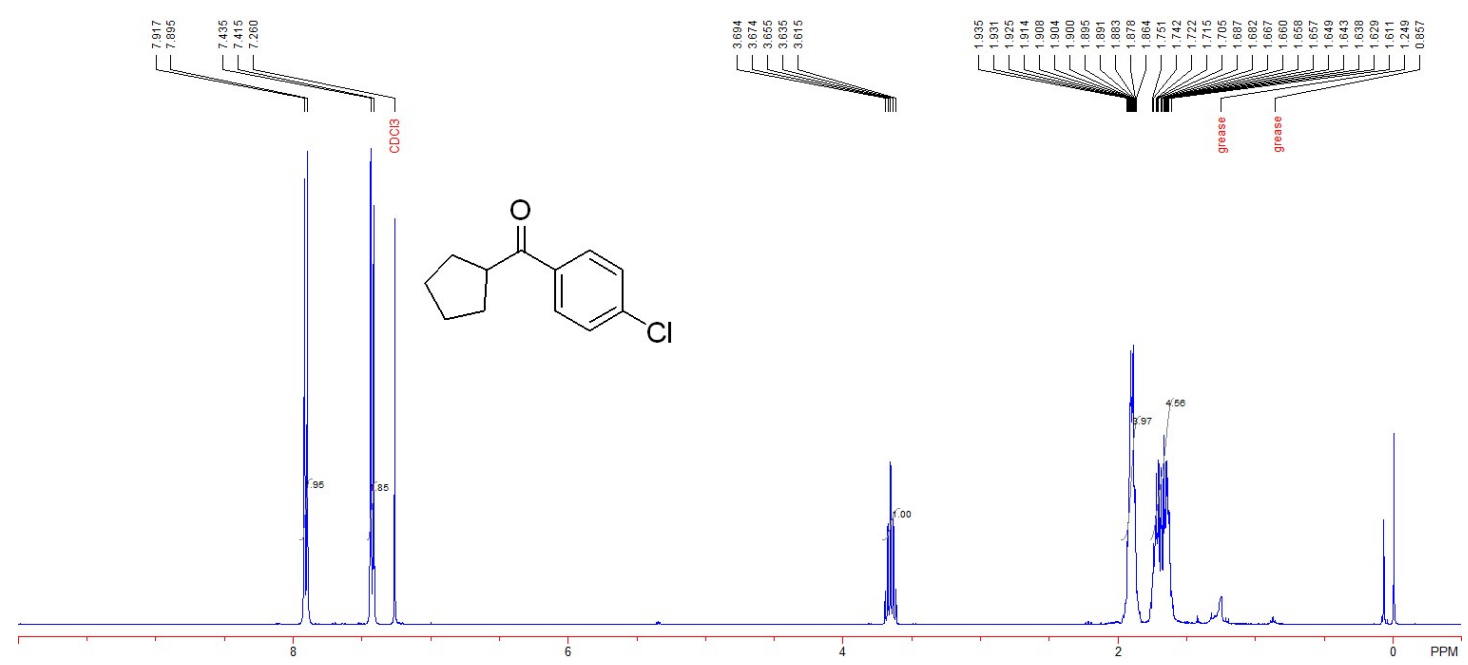

\section{Compound 4w}

${ }^{13} \mathrm{C} \mathrm{NMR}\left(101 \mathrm{MHz}, \mathrm{CDCl}_{3}\right)$

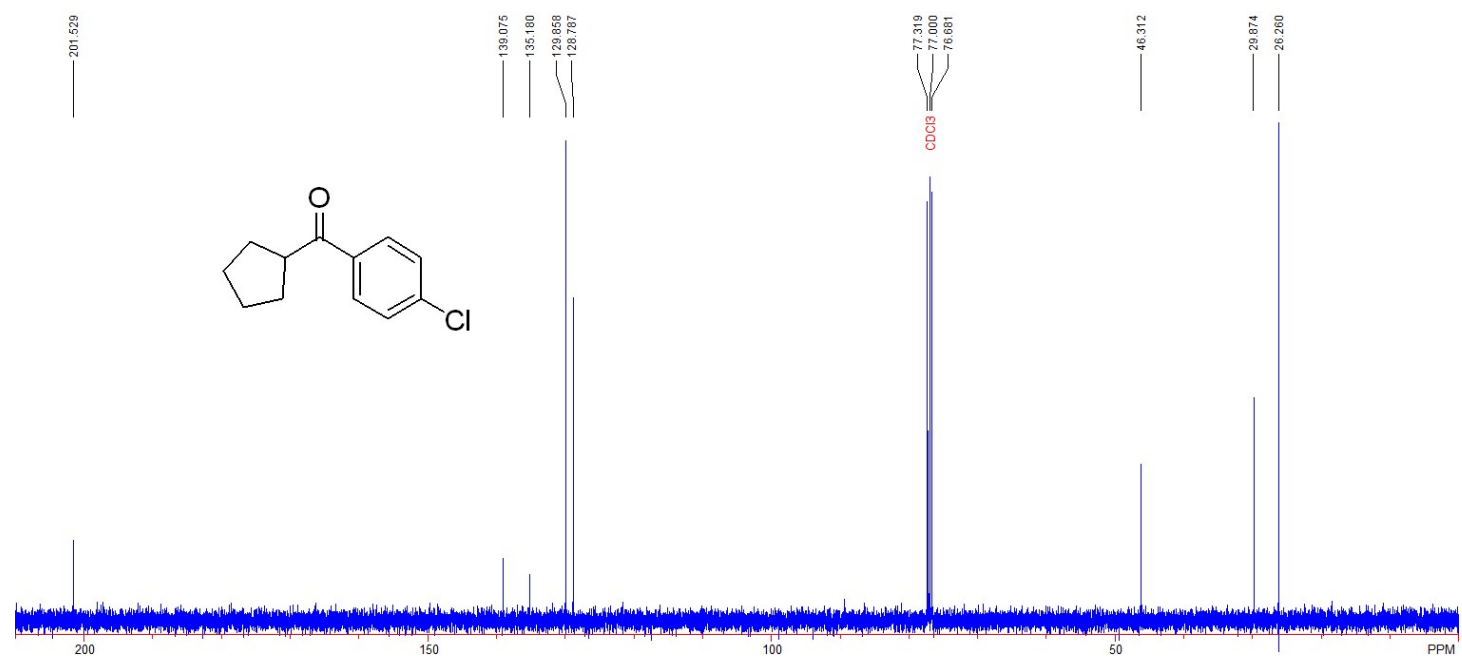




\section{Compound 4x}

${ }^{1} \mathrm{H}$ NMR (400 MHz, $\mathrm{CDCl}_{3}$ )

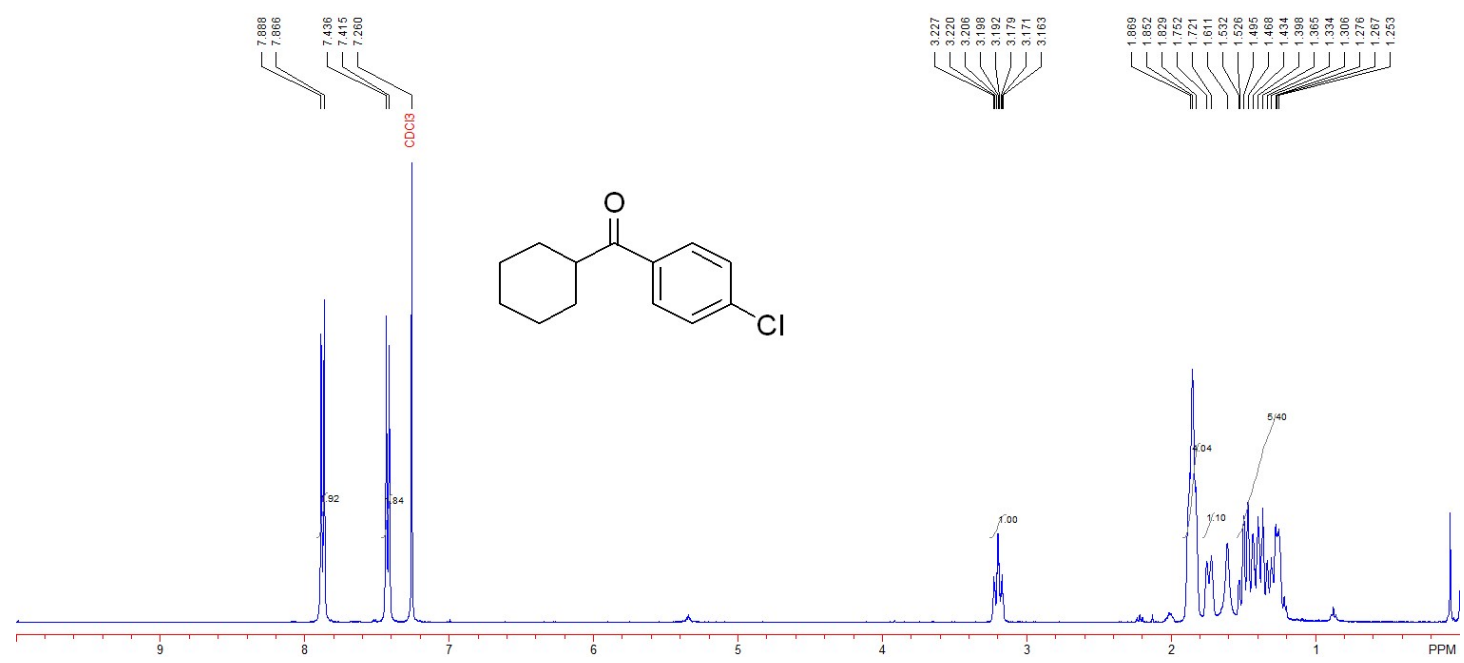

\section{Compound 4x}

${ }^{13} \mathrm{C} \mathrm{NMR}\left(101 \mathrm{MHz}, \mathrm{CDCl}_{3}\right)$

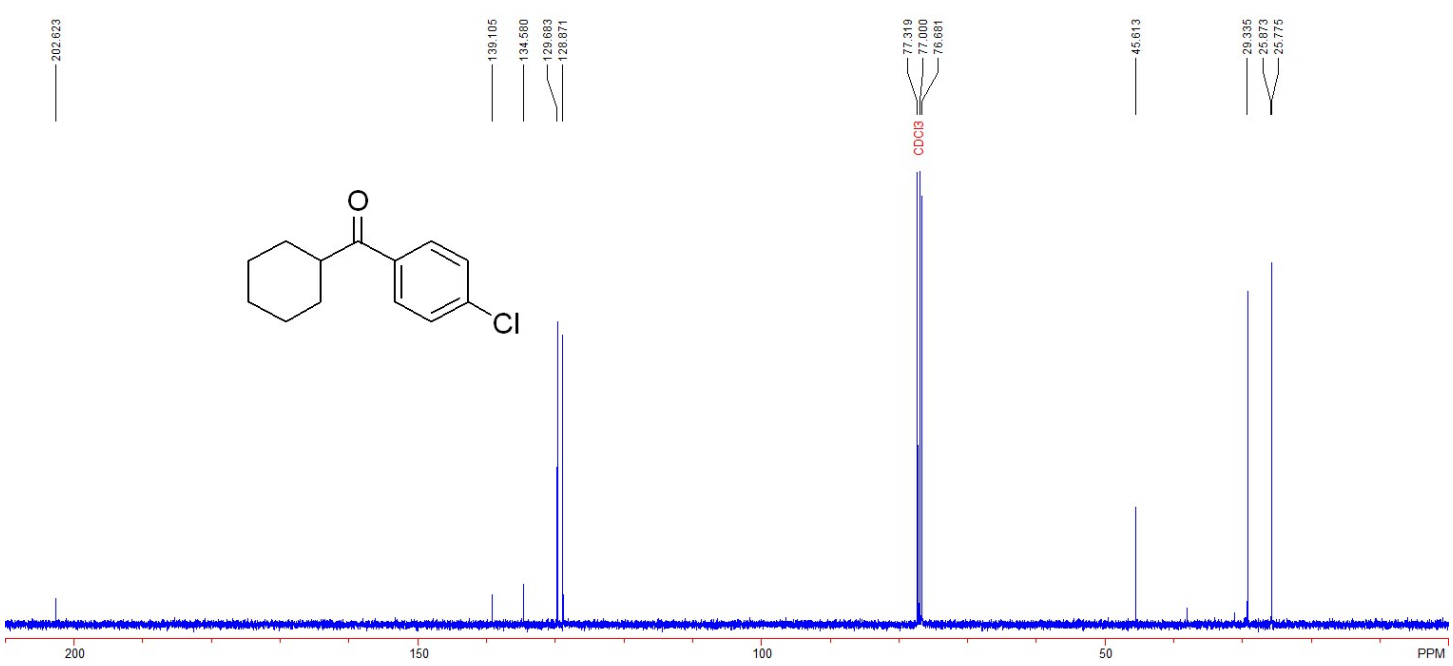


Compound $4 y$-one isomer

${ }^{1} \mathrm{H}$ NMR (400 MHz, $\left.\mathrm{CDCl}_{3}\right)$
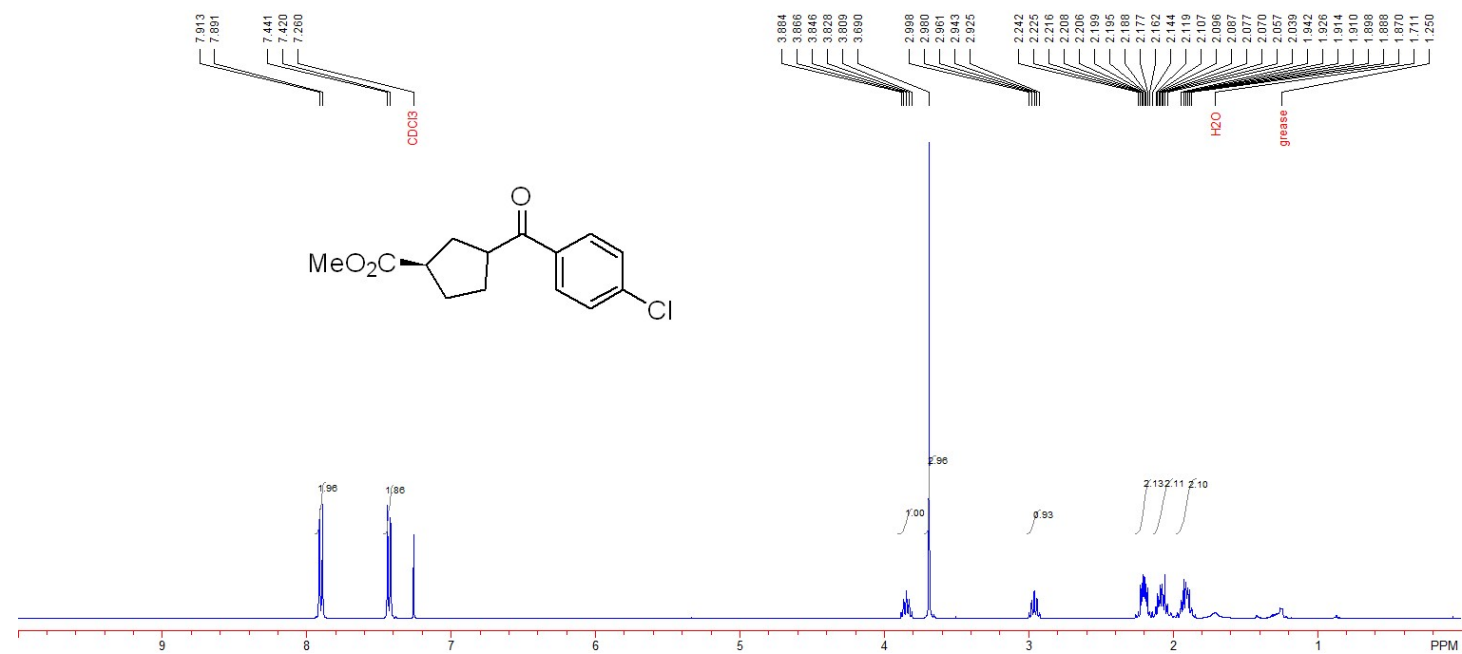

\section{Compound $4 y$-one isomer}

${ }^{13} \mathrm{C} \mathrm{NMR}\left(101 \mathrm{MHz}, \mathrm{CDCl}_{3}\right)$

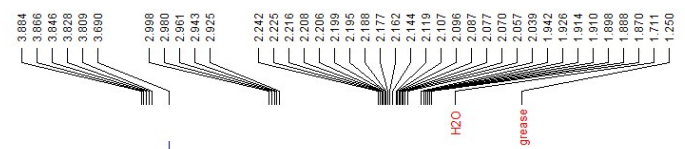

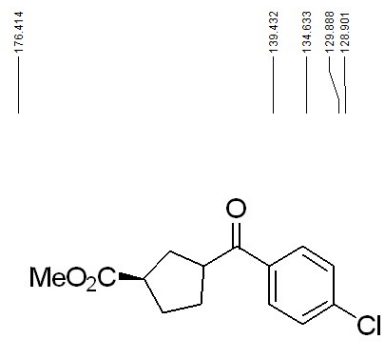
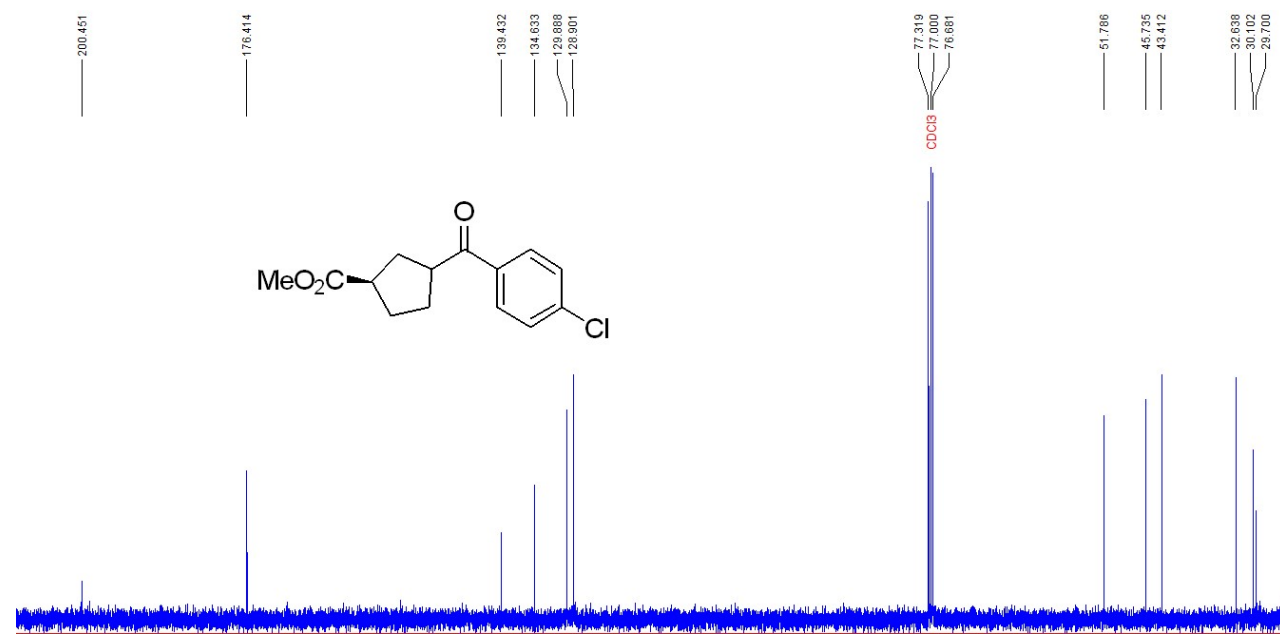
Compound $4 y$-the other isomer

${ }^{1} \mathrm{H}$ NMR (400 MHz, $\left.\mathrm{CDCl}_{3}\right)$

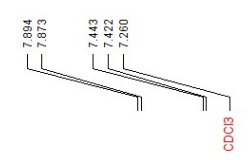<smiles>COC(=O)C1CCC(C(=O)c2ccc(Cl)cc2)C1</smiles>

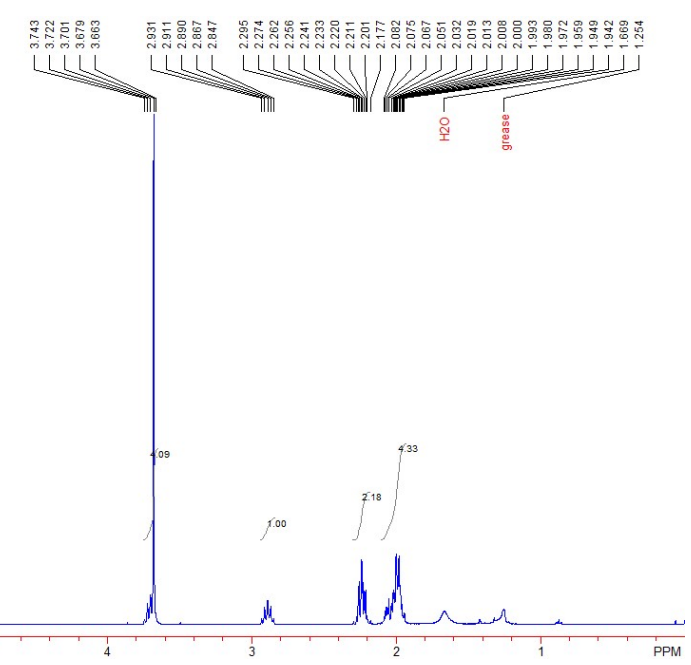

\section{Compound $4 y$-the other isomer}

${ }^{13} \mathrm{C} \mathrm{NMR}\left(101 \mathrm{MHz}, \mathrm{CDCl}_{3}\right)$
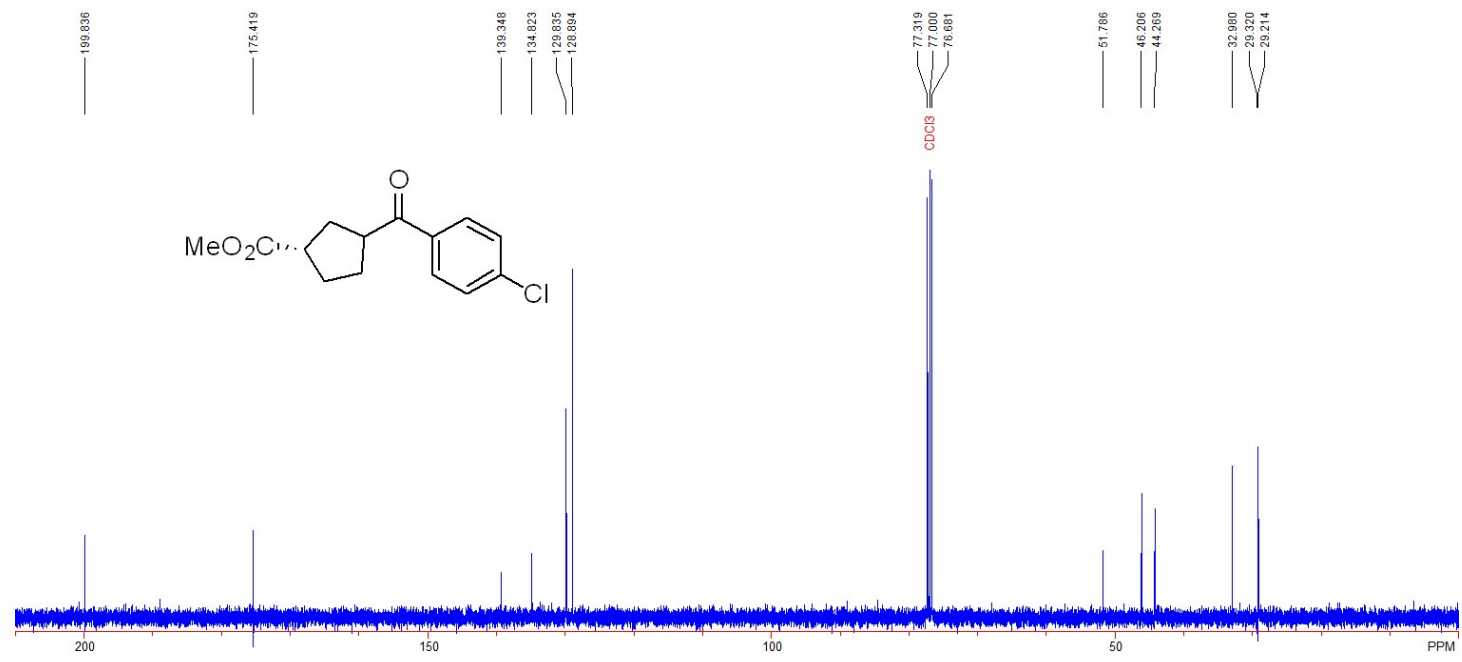


\section{Compound 4za}

${ }^{1} \mathrm{H}$ NMR (400 MHz, $\mathrm{CDCl}_{3}$ )

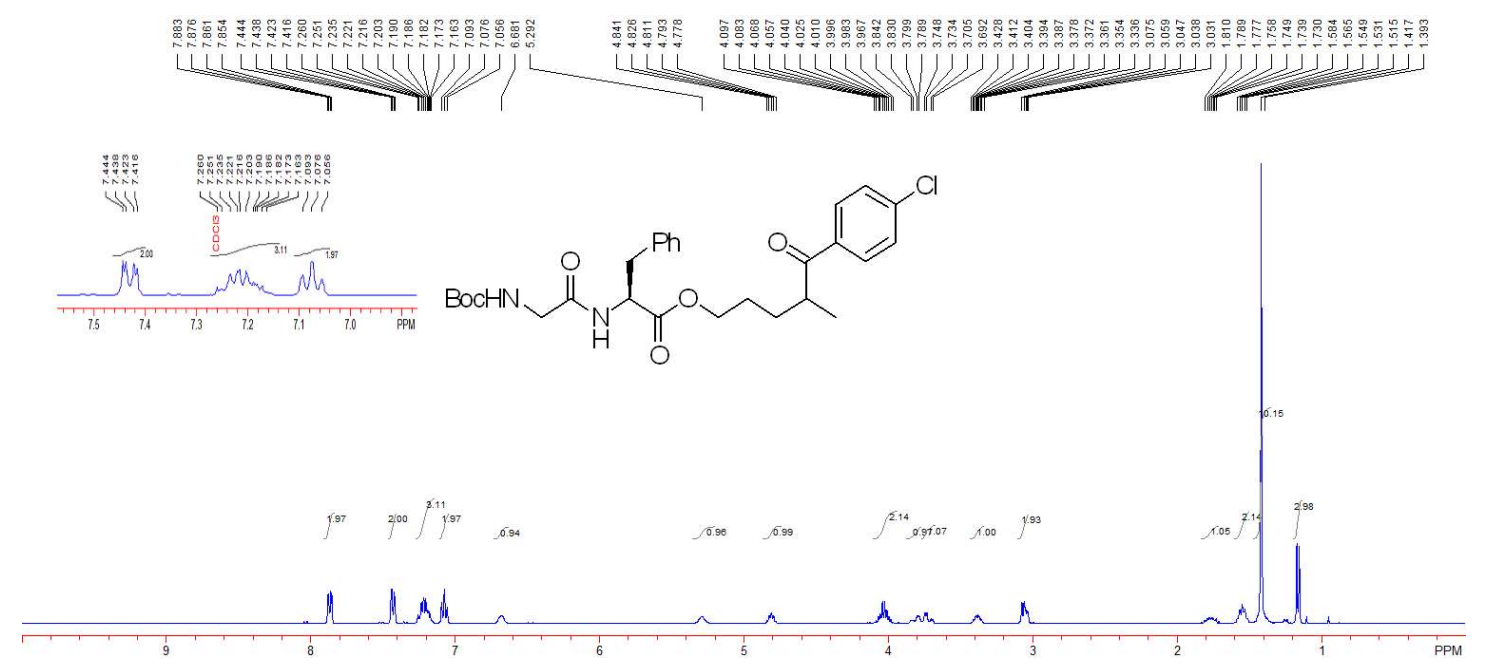

\section{Compound 4za}

${ }^{13} \mathrm{C} \mathrm{NMR}\left(101 \mathrm{MHz}, \mathrm{CDCl}_{3}\right)$

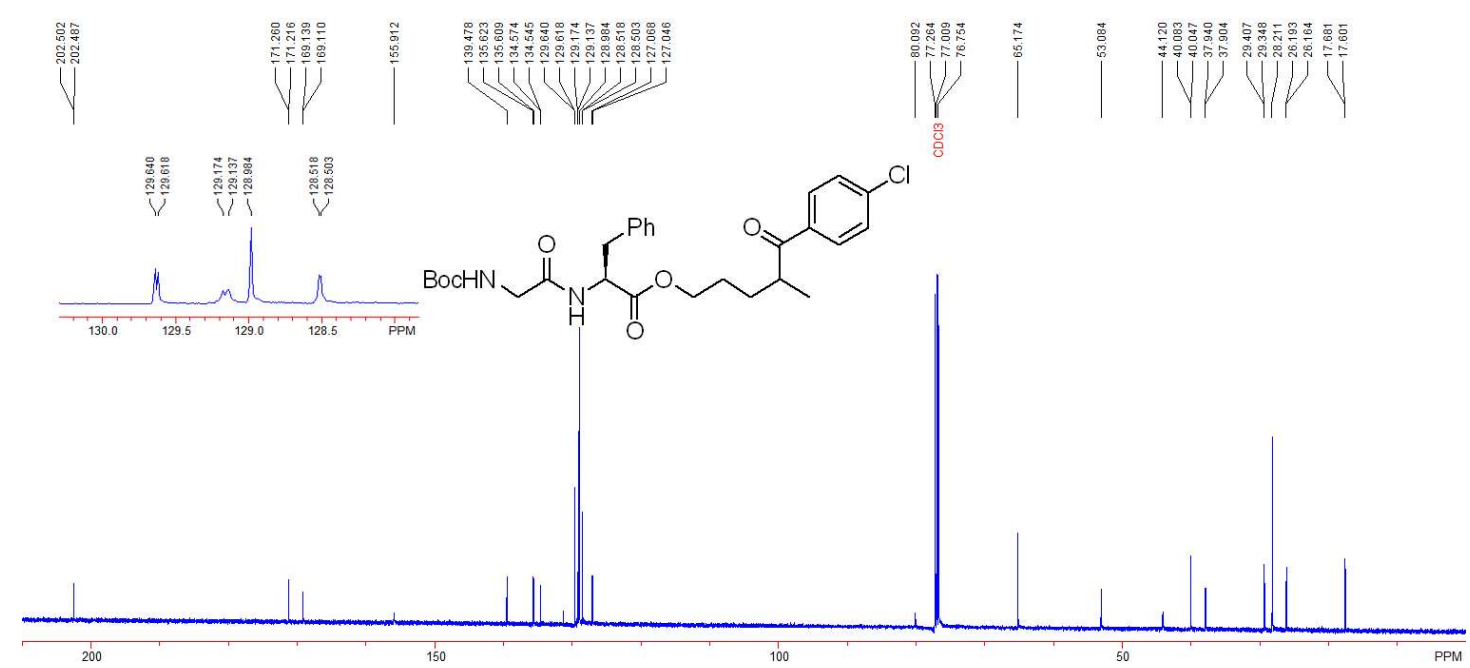




\section{Compound 4zb}

${ }^{1} \mathrm{H}$ NMR (400 MHz, $\mathrm{CDCl}_{3}$ )

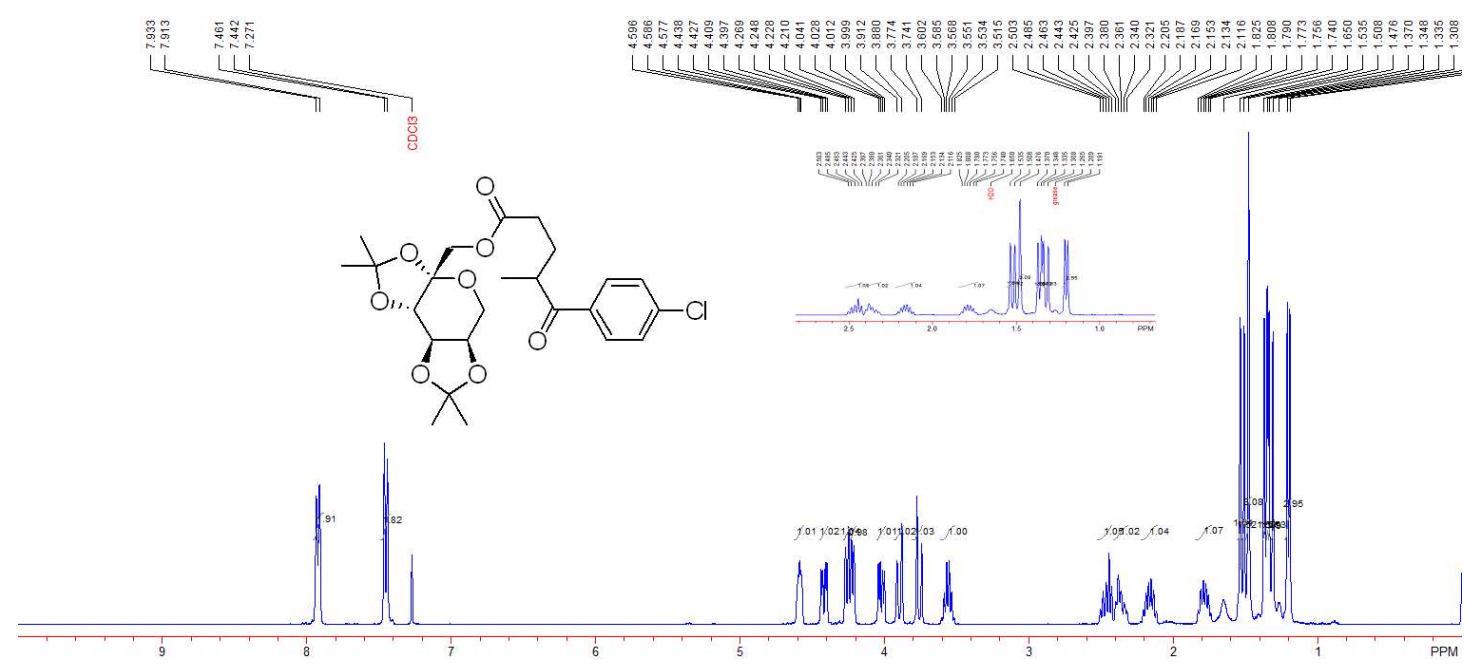

\section{Compound 4zb}

${ }^{13} \mathrm{C} \mathrm{NMR}\left(101 \mathrm{MHz}, \mathrm{CDCl}_{3}\right)$
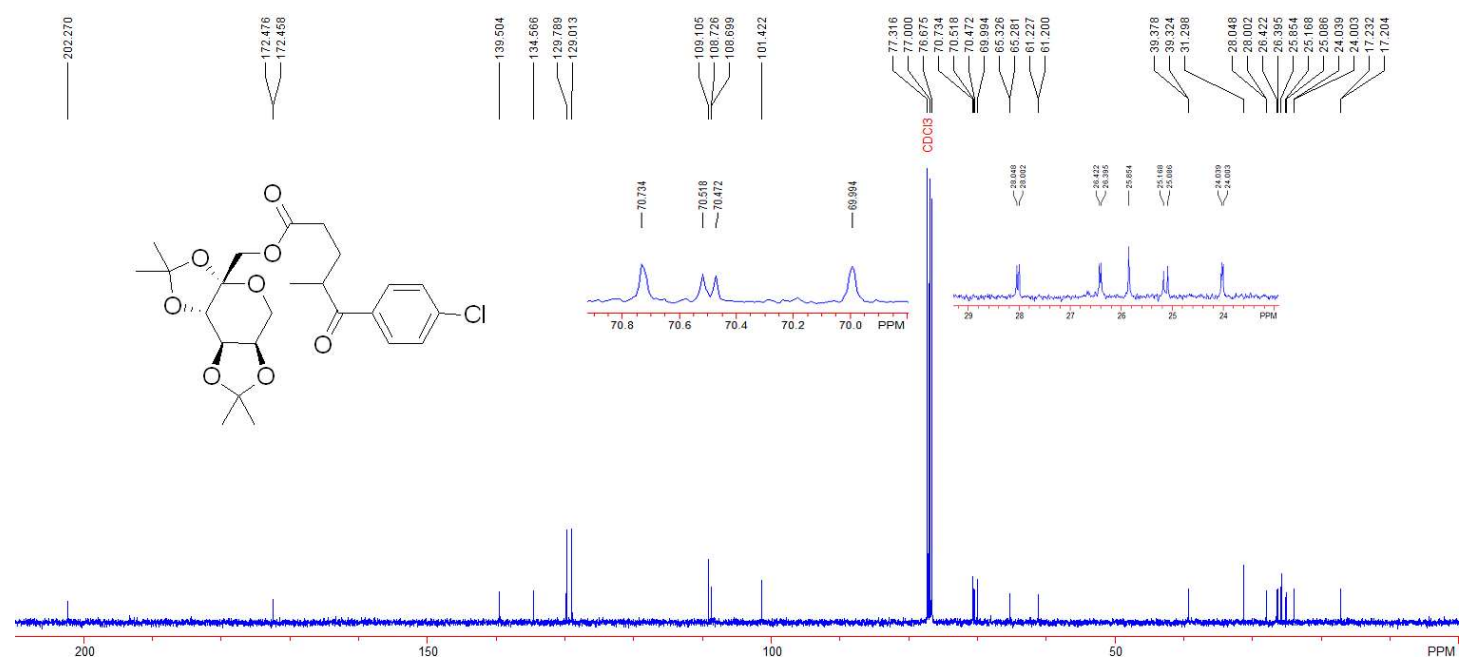


\section{Compound 4ze}

${ }^{1} \mathrm{H}$ NMR (400 MHz, $\left.\mathrm{CDCl}_{3}\right)$

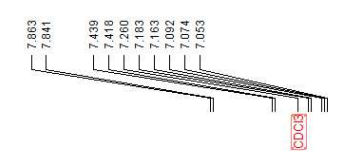

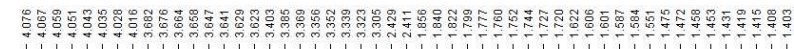
LILLLLLUW LIL
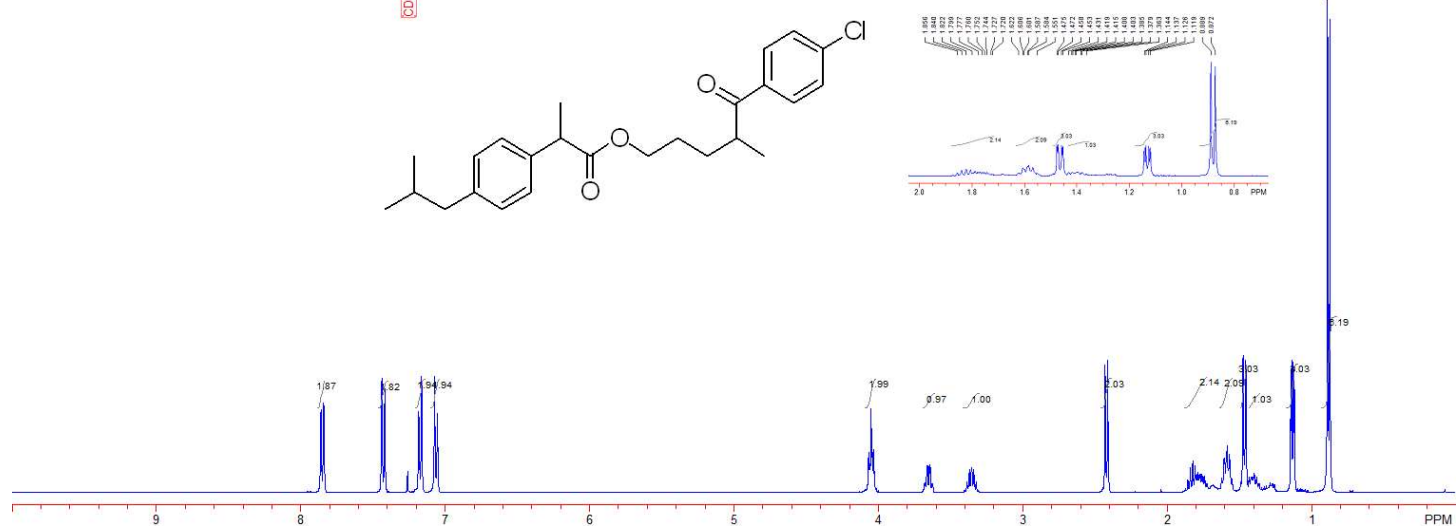

\section{Compound 4zc}

${ }^{13} \mathrm{C}$ NMR (101 MHz, $\left.\mathrm{CDCl}_{3}\right)$
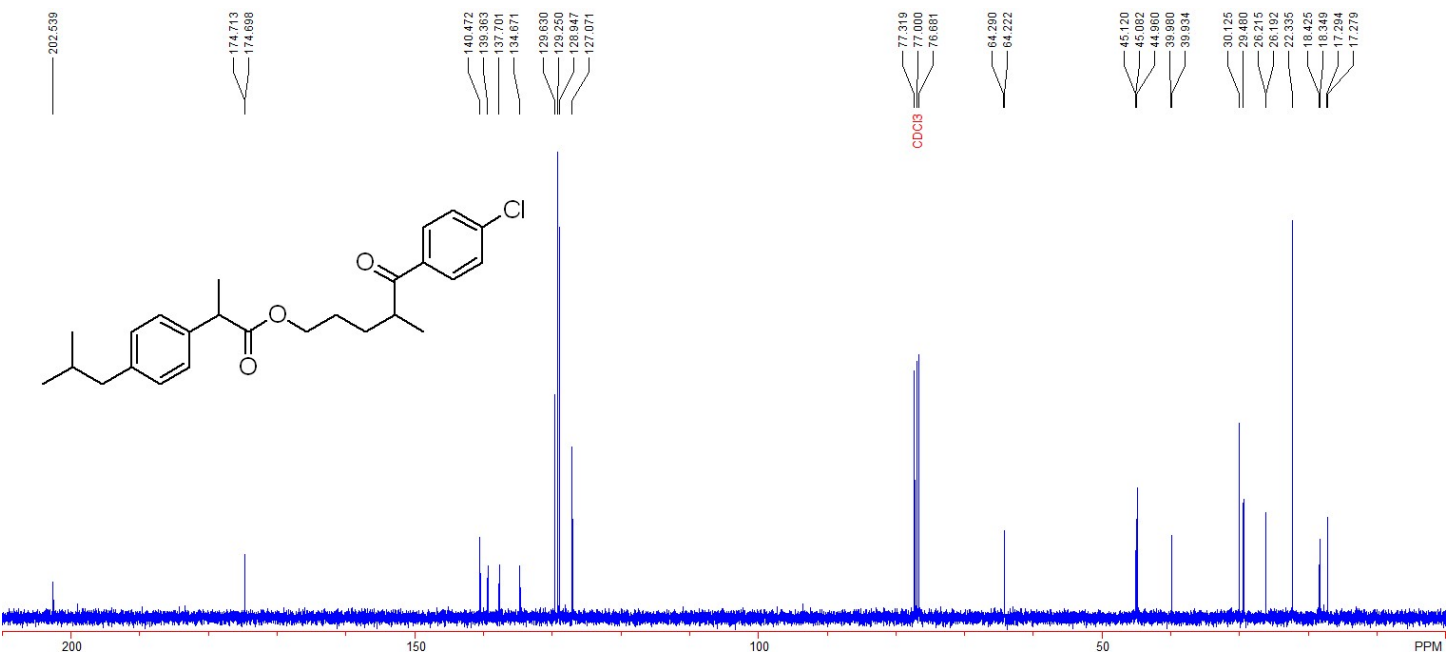


\section{Compound 4zd}

${ }^{1} \mathrm{H}$ NMR (400 MHz, $\mathrm{CDCl}_{3}$ )

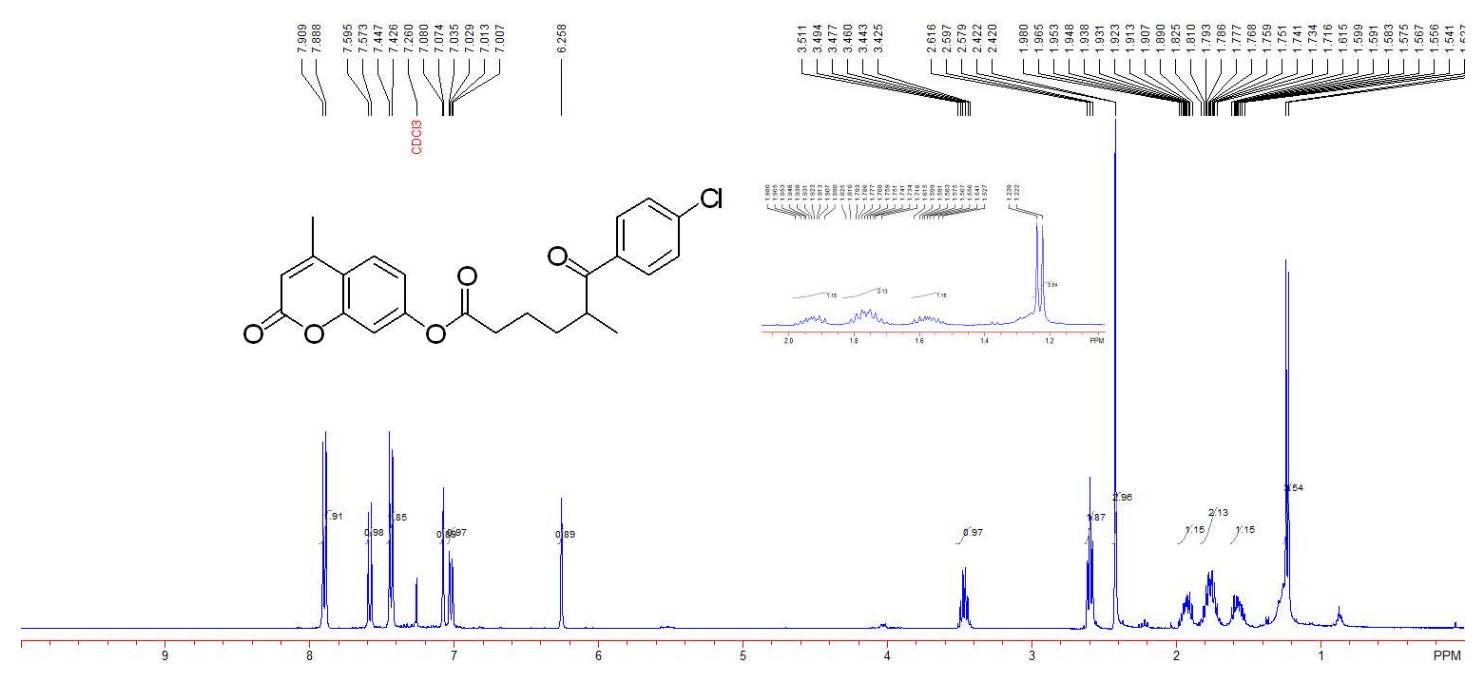

\section{Compound 4zd}

${ }^{13} \mathrm{C} \mathrm{NMR}\left(101 \mathrm{MHz}, \mathrm{CDCl}_{3}\right)$

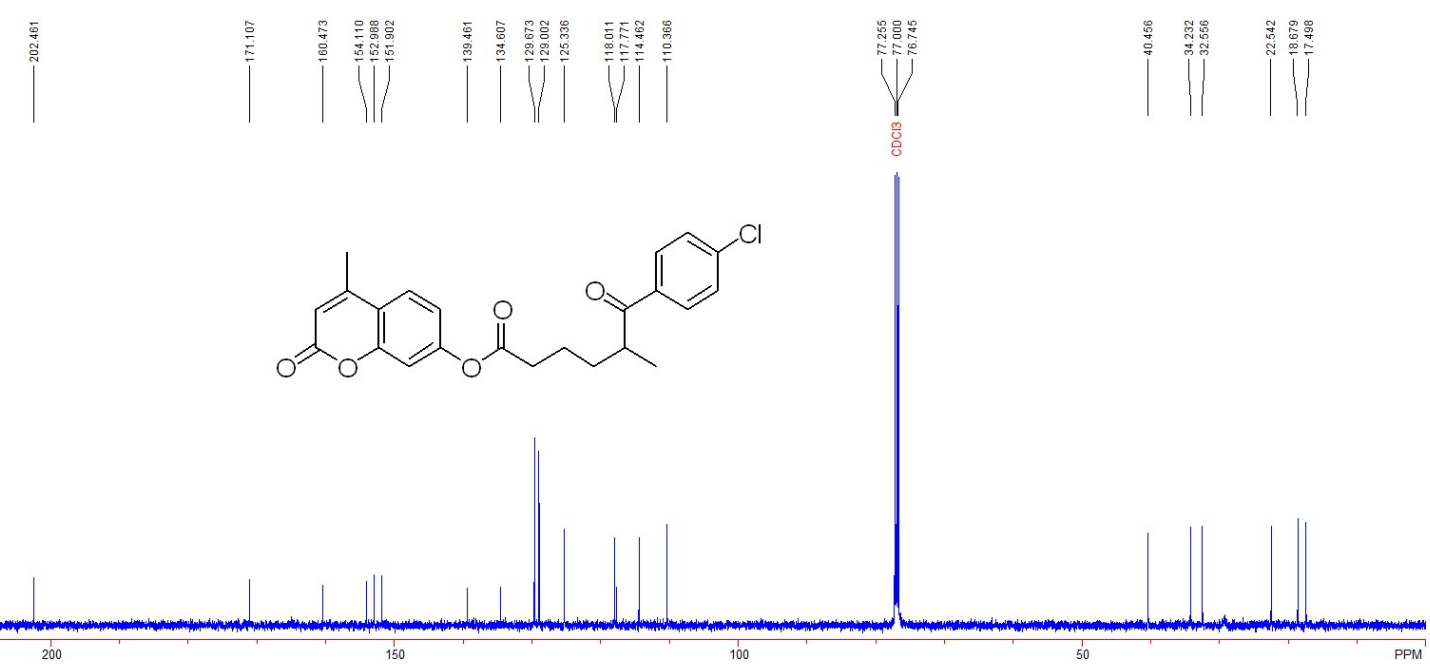




\section{Compound 4ze}

${ }^{1} \mathrm{H}$ NMR (400 MHz, $\mathrm{CDCl}_{3}$ )

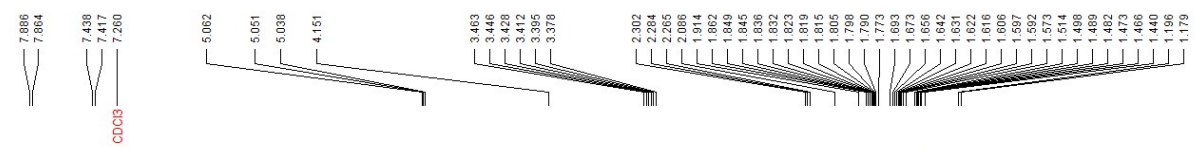<smiles>CC(CCCC(=O)OC1CC2CCC(C1)[N+]2([O-])c1ccccc1)C(=O)c1ccc(Cl)cc1</smiles>

\section{Compound 4ze}

${ }^{13} \mathrm{C} \mathrm{NMR}\left(101 \mathrm{MHz}, \mathrm{CDCl}_{3}\right)$
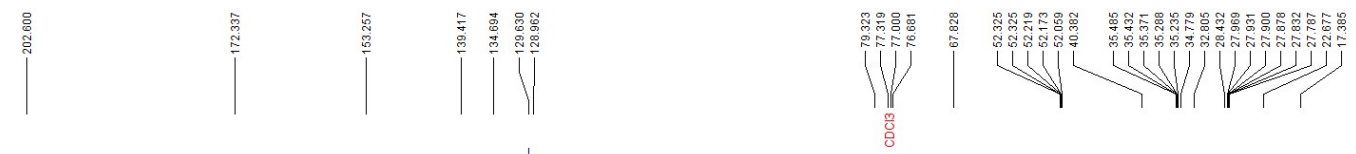<smiles>CC(CCCC(=O)OC1CC2CCC(C1)N2C(=O)c1ccccc1)C(=O)c1ccc(Cl)cc1</smiles>

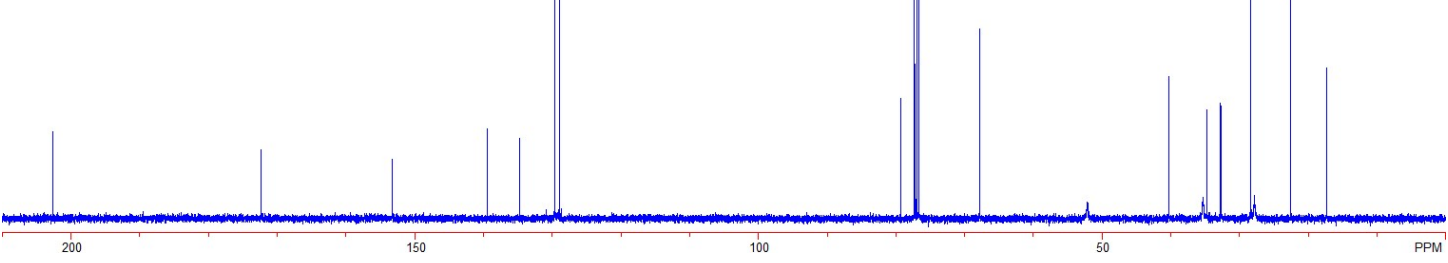




\section{Compound 4zf}

${ }^{1} \mathrm{H}$ NMR (400 MHz, $\mathrm{CDCl}_{3}$ )
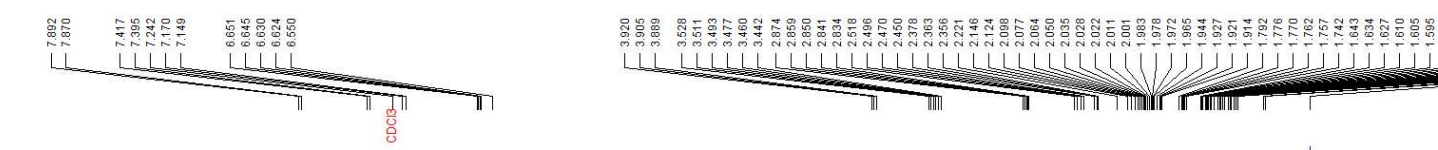

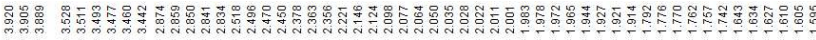

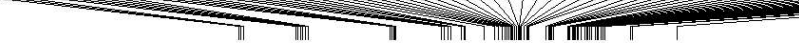

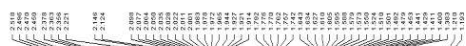
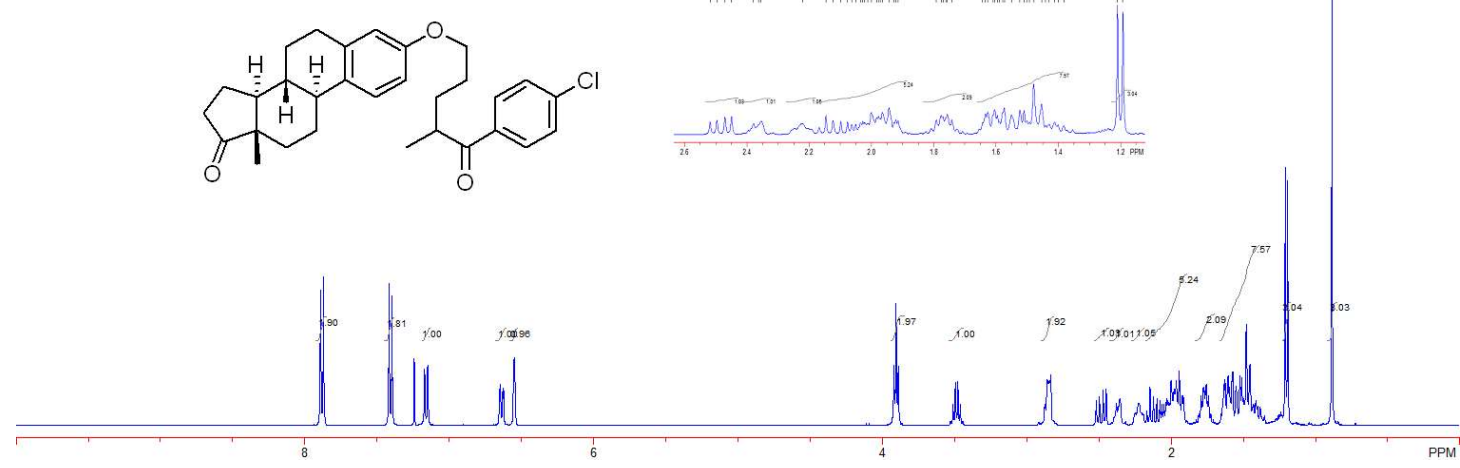

\section{Compound 4zf}

${ }^{13} \mathrm{C}$ NMR (101 MHz, $\left.\mathrm{CDCl}_{3}\right)$
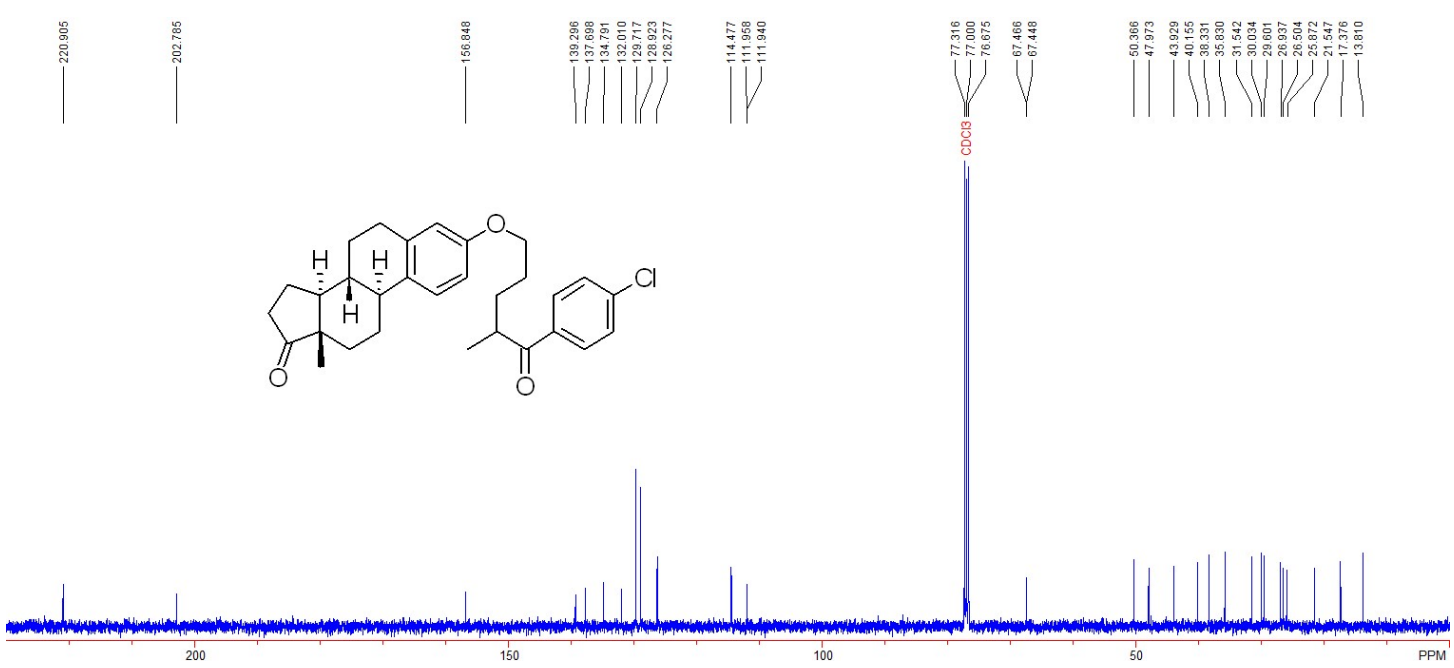


\section{Compound 6a}

${ }^{1} \mathrm{H} \mathrm{NMR}\left(400 \mathrm{MHz}, \mathrm{CDCl}_{3}\right)$

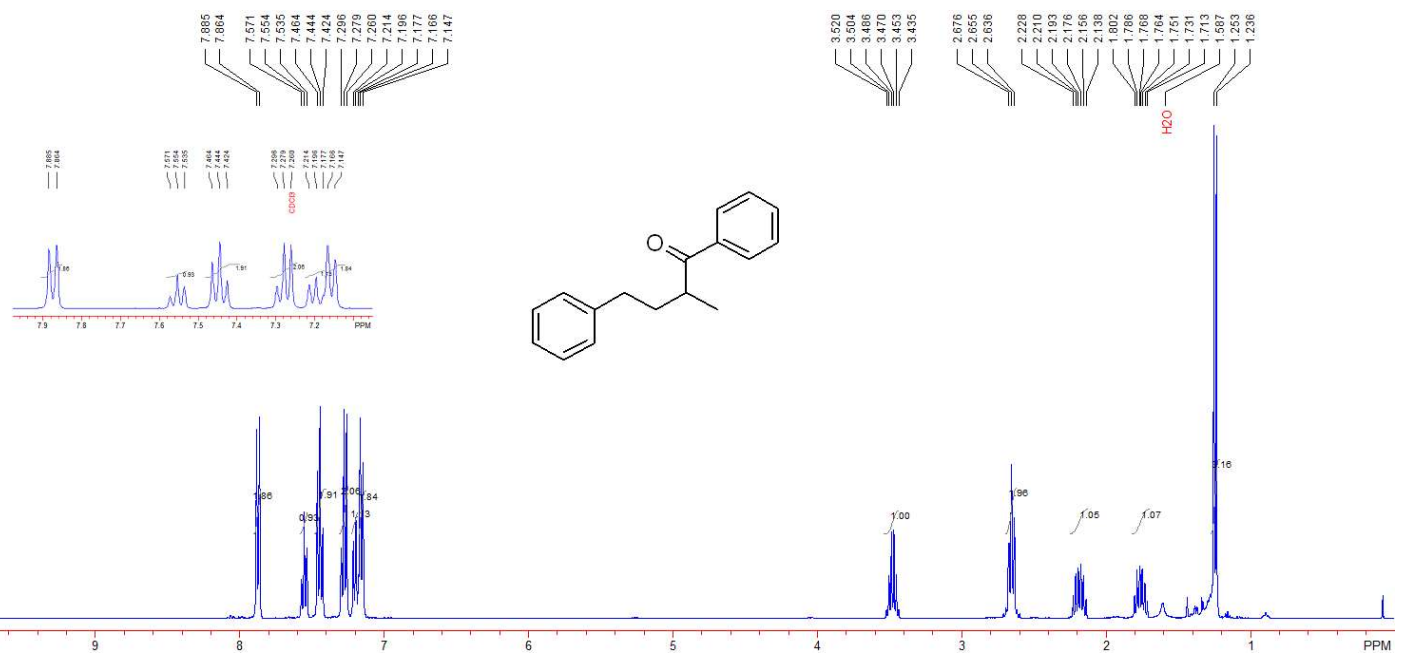

\section{Compound 6a}

${ }^{13} \mathrm{C} \mathrm{NMR}\left(101 \mathrm{MHz}, \mathrm{CDCl}_{3}\right)$

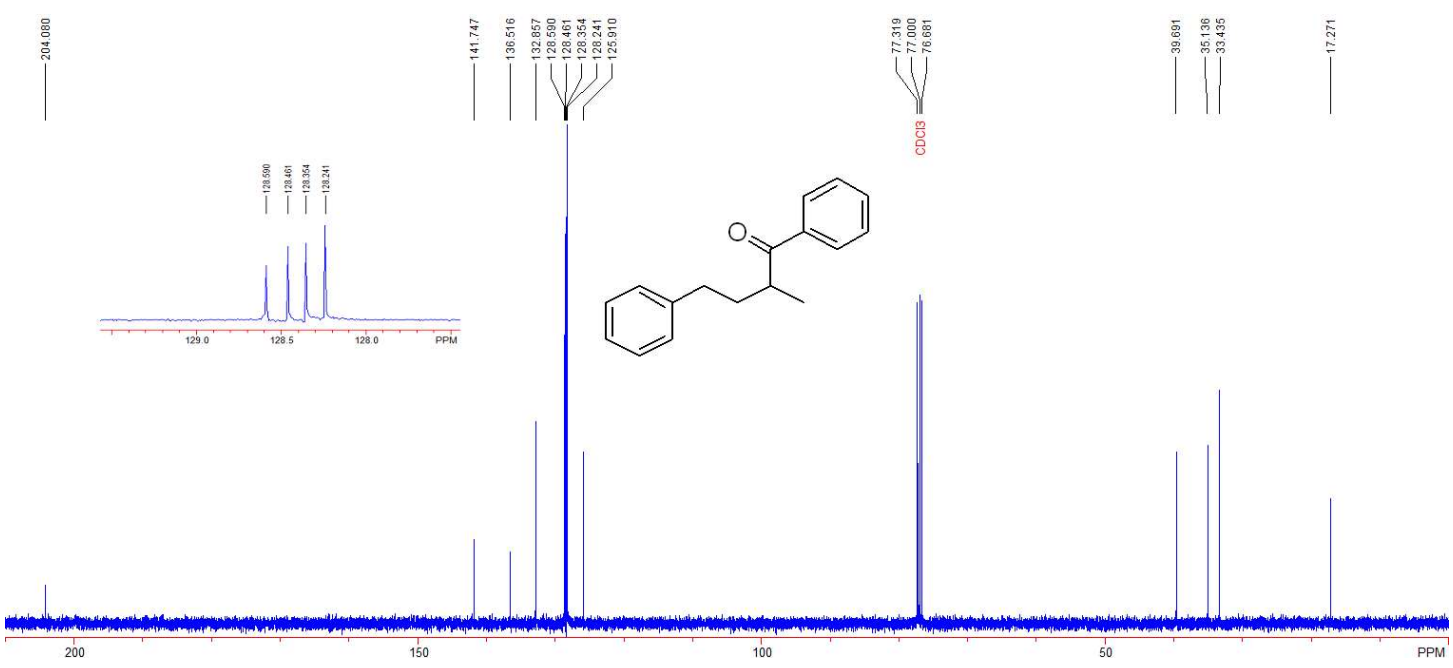


Compound bb ${ }^{1} \mathrm{H}$ NMR (400 MHz, $\mathrm{CDCl}_{3}$ )
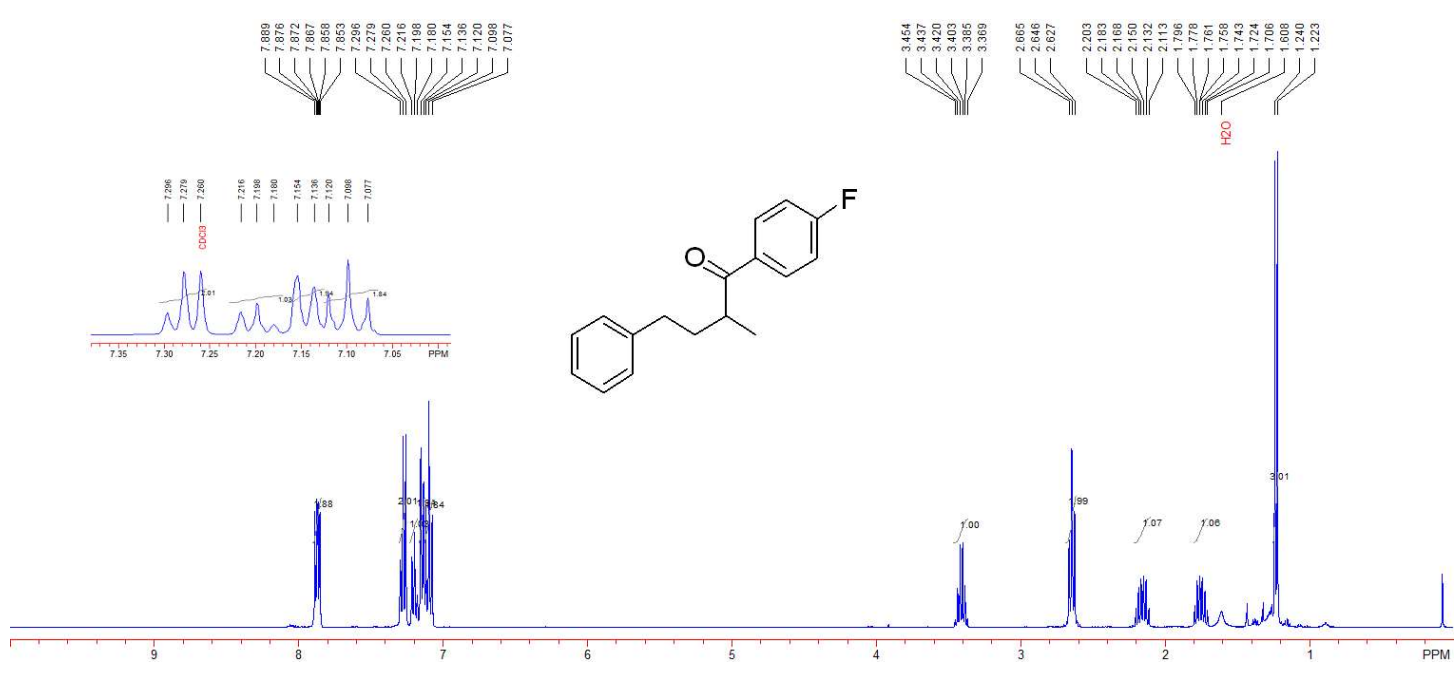

Compound bb ${ }^{19} \mathrm{~F}$ NMR (376 MHz, $\mathrm{CDCl}_{3}$ )
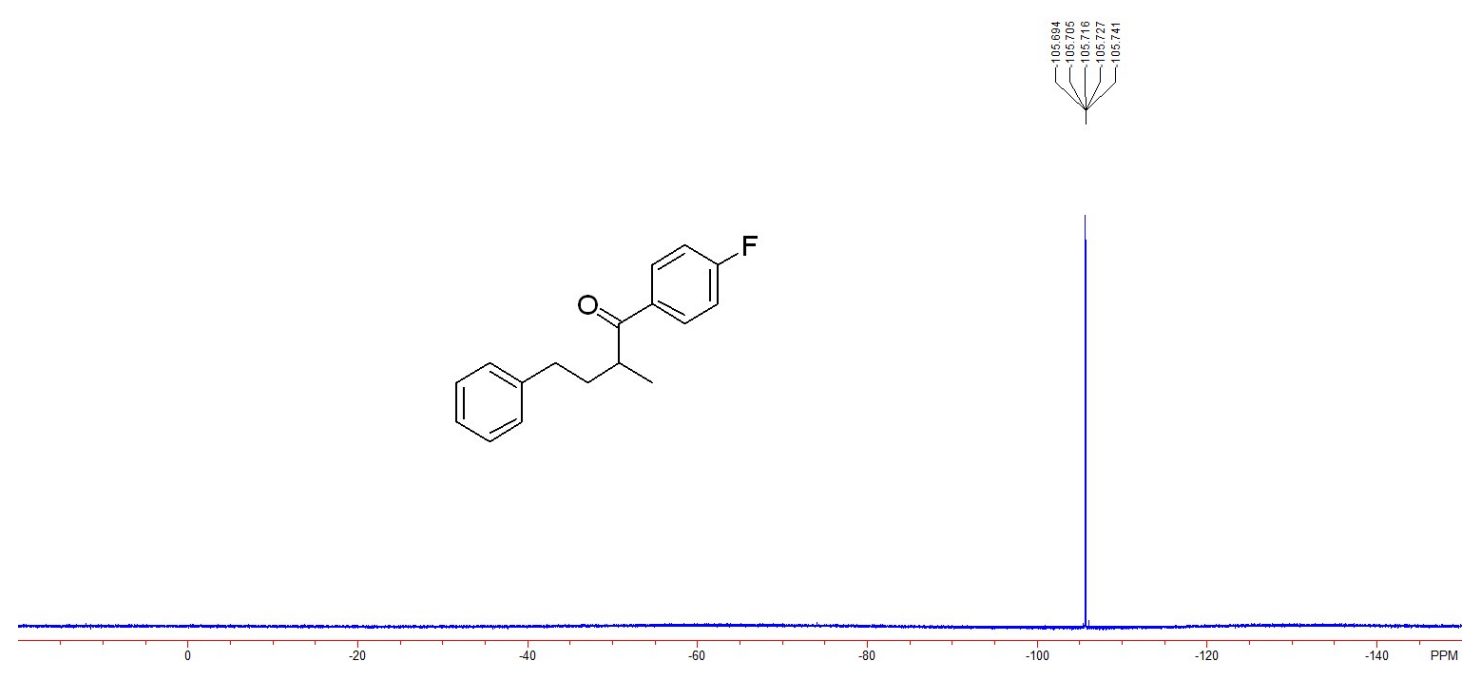

Compound $6 \mathbf{b}{ }^{13} \mathrm{C}$ NMR (101 MHz, $\left.\mathrm{CDCl}_{3}\right)$
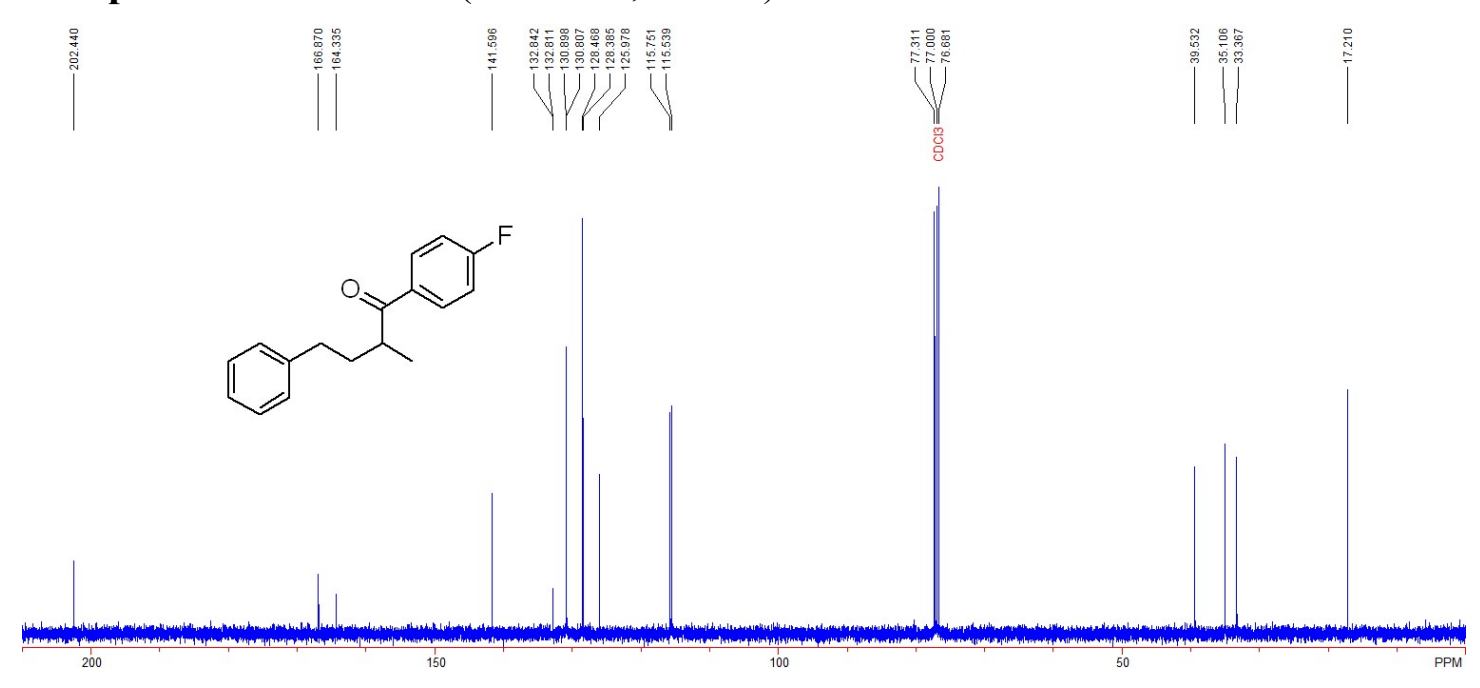

S107 


\section{Compound 6c}

${ }^{1} \mathrm{H}$ NMR (400 MHz, $\left.\mathrm{CDCl}_{3}\right)$

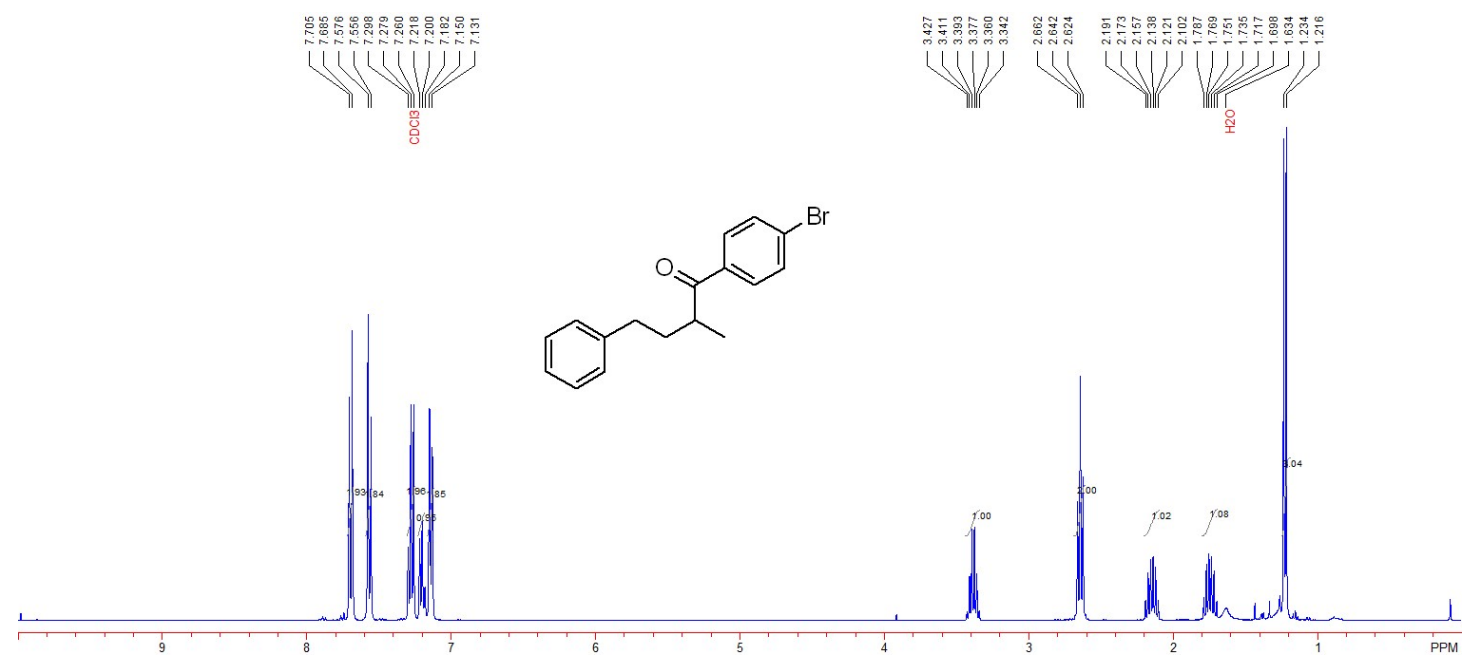

\section{Compound 6c}

${ }^{13} \mathrm{C} \mathrm{NMR}\left(101 \mathrm{MHz}, \mathrm{CDCl}_{3}\right)$

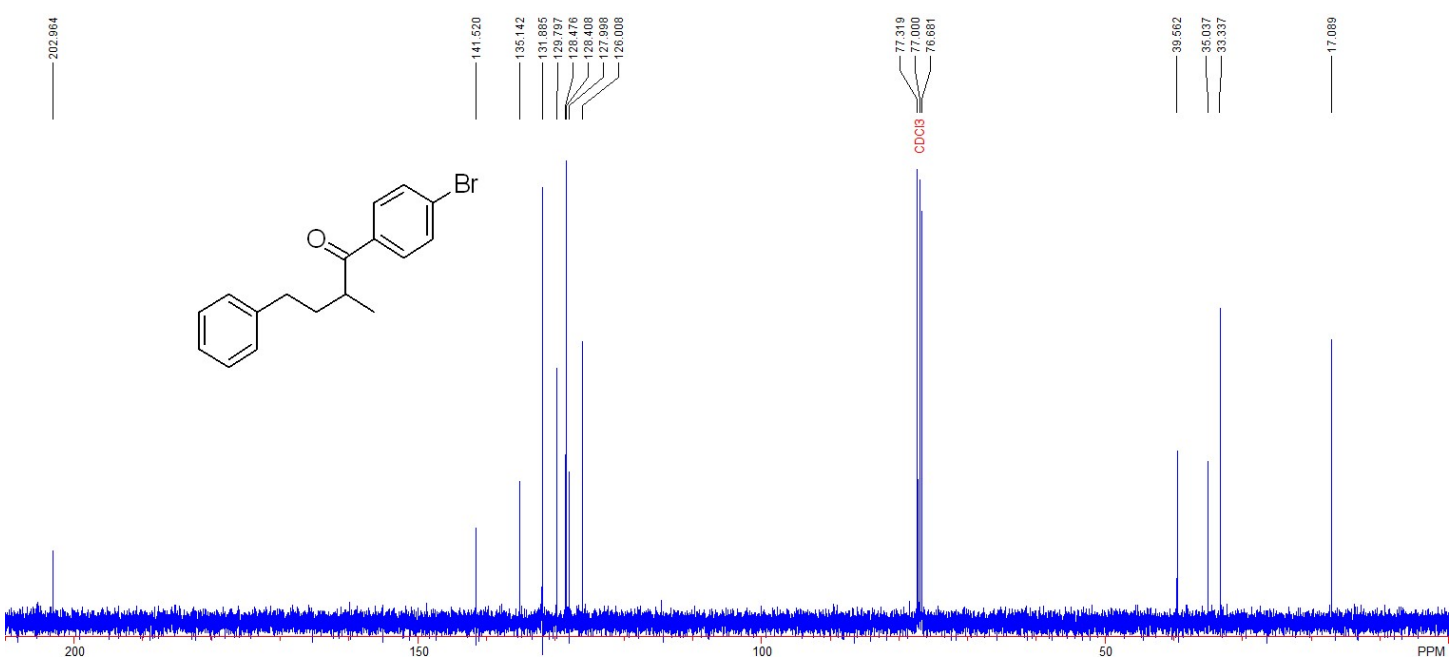




\section{Compound 6d}

${ }^{1} \mathrm{H}$ NMR (400 MHz, $\left.\mathrm{CDCl}_{3}\right)$

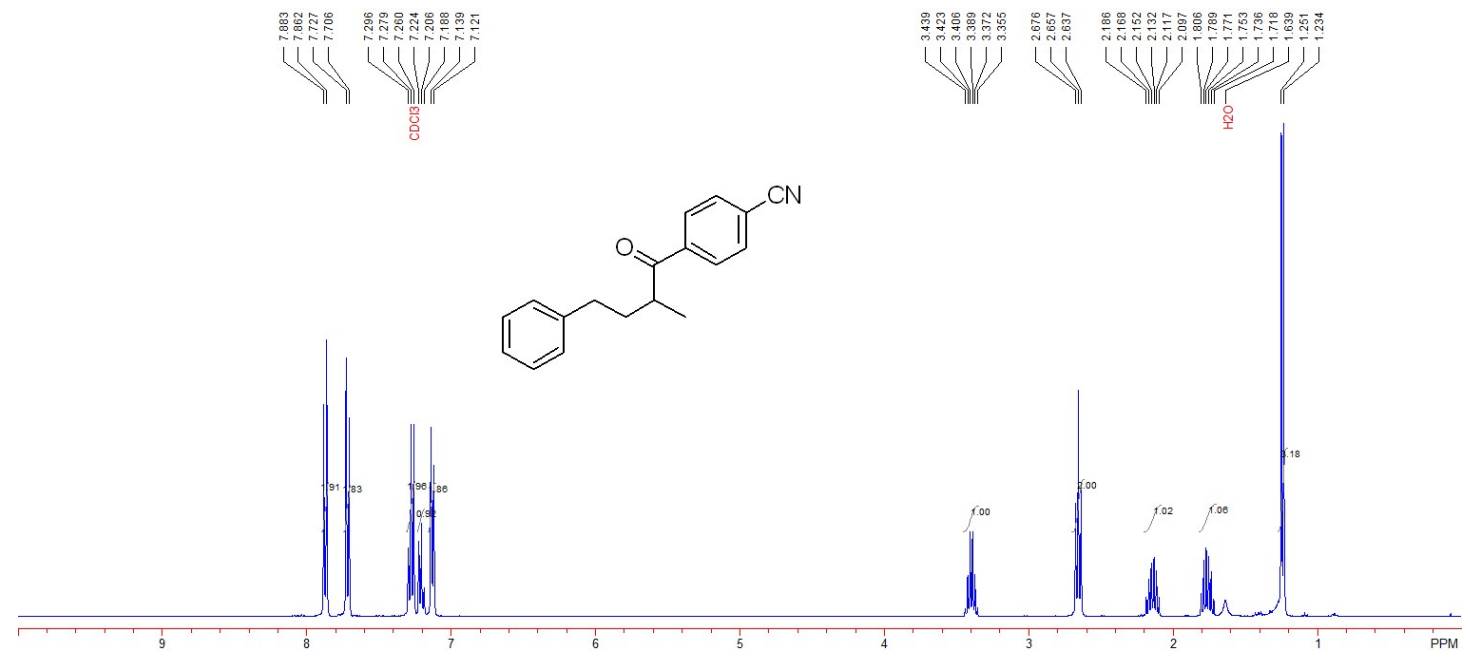

\section{Compound 6d}

${ }^{13} \mathrm{C} \mathrm{NMR}\left(101 \mathrm{MHz}, \mathrm{CDCl}_{3}\right)$

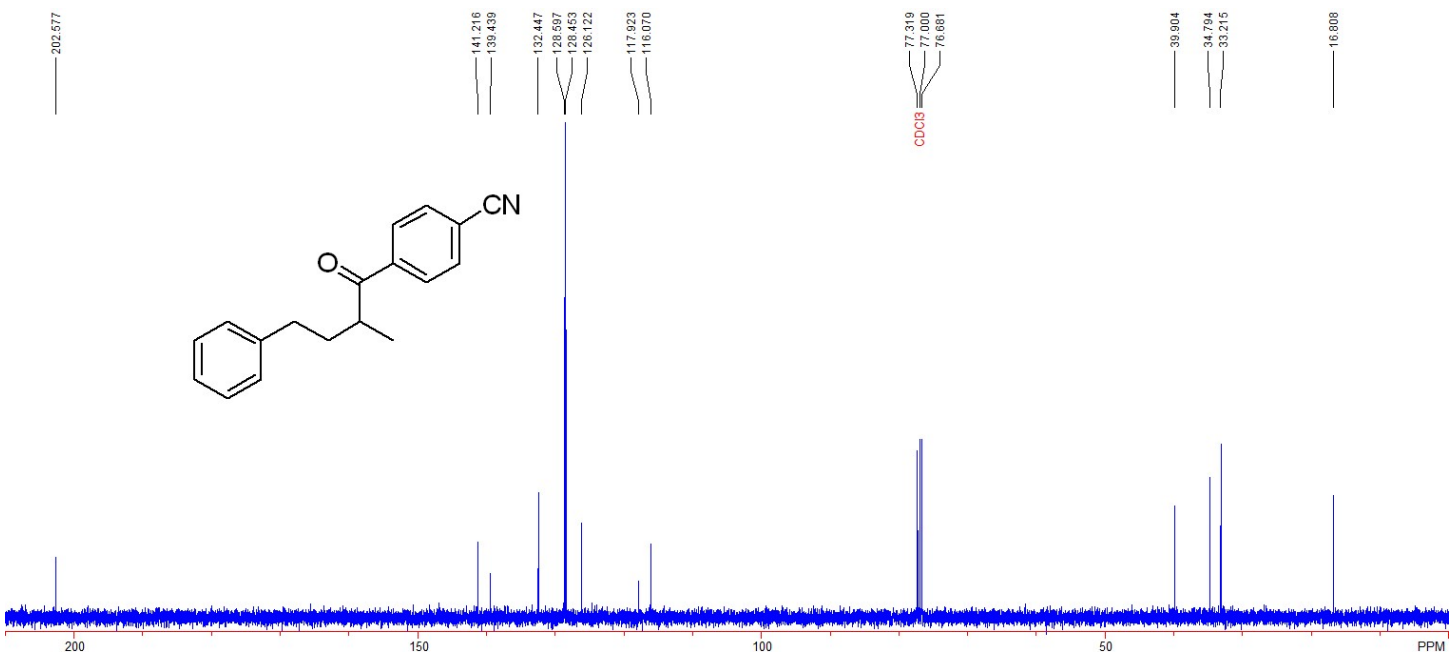




\section{Compound 6e}

${ }^{1} \mathrm{H}$ NMR (400 MHz, $\mathrm{CDCl}_{3}$ )

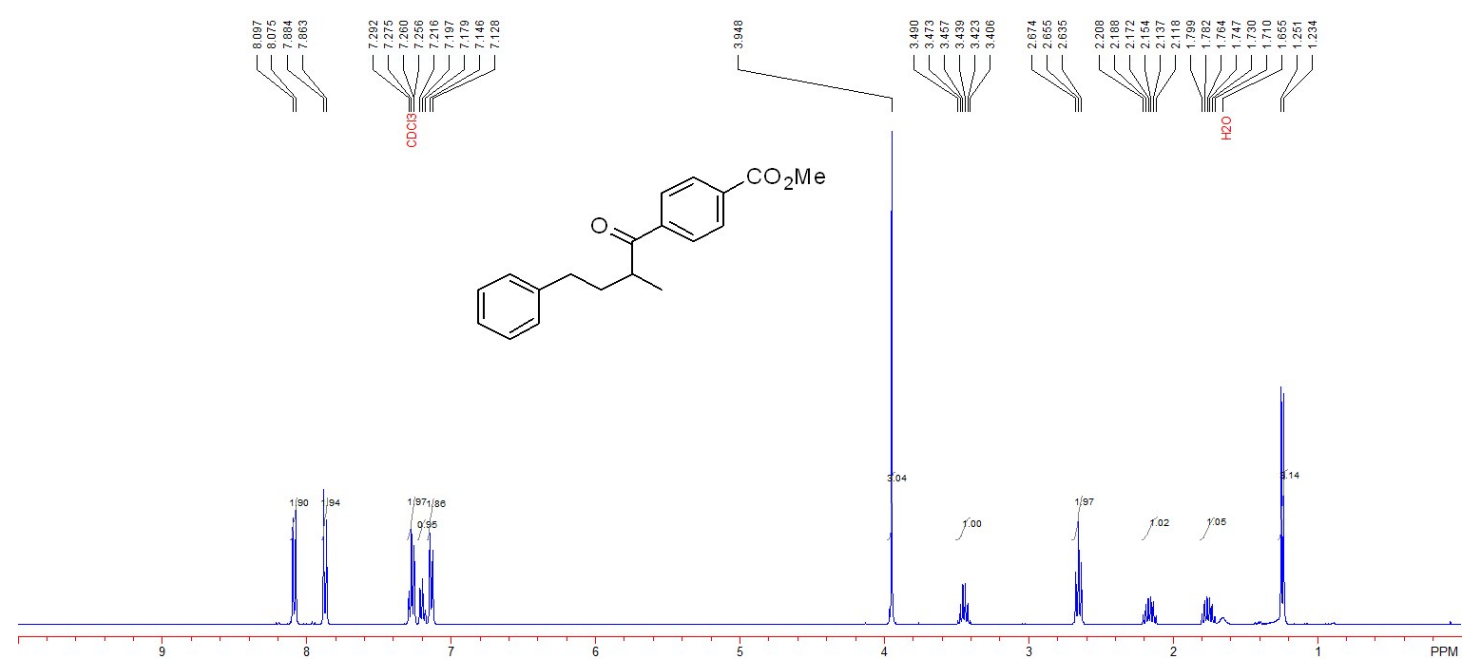

\section{Compound 6e}

${ }^{13} \mathrm{C} \mathrm{NMR}\left(101 \mathrm{MHz}, \mathrm{CDCl}_{3}\right)$

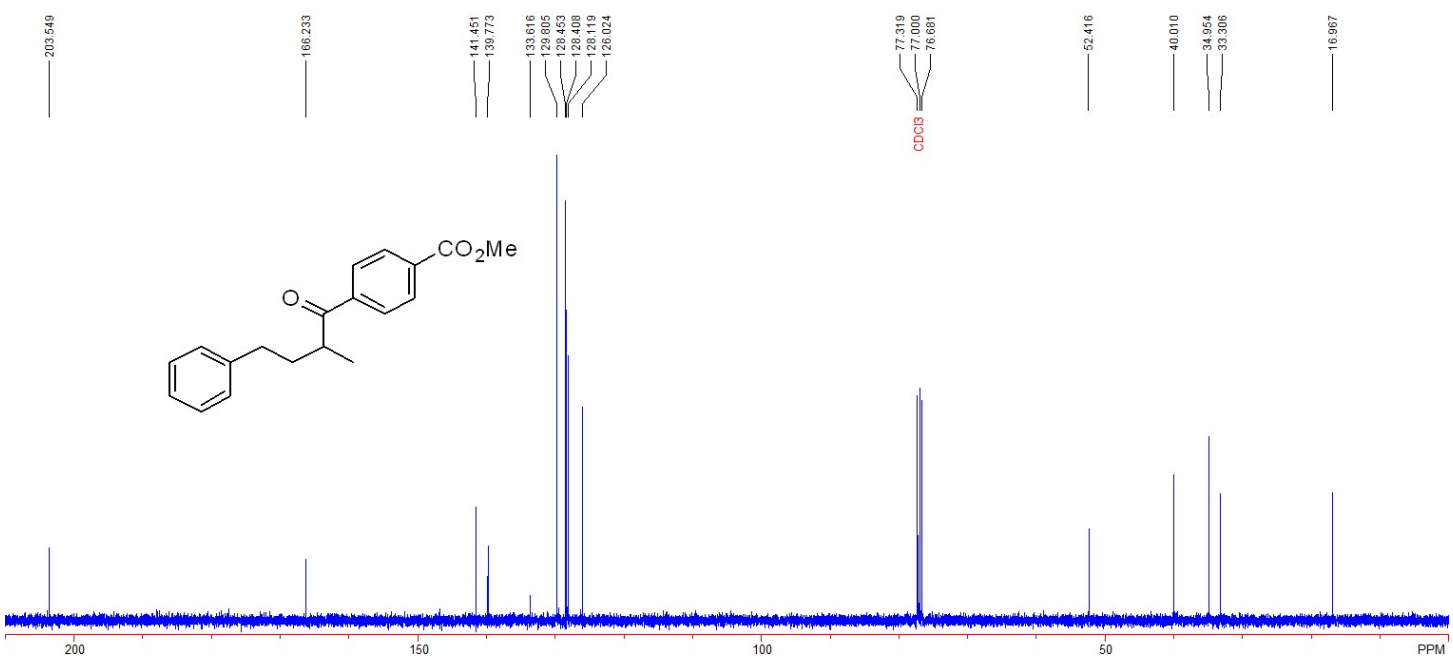


Compound $6{ }^{1} \mathrm{H}$ NMR (400 MHz, $\mathrm{CDCl}_{3}$ )
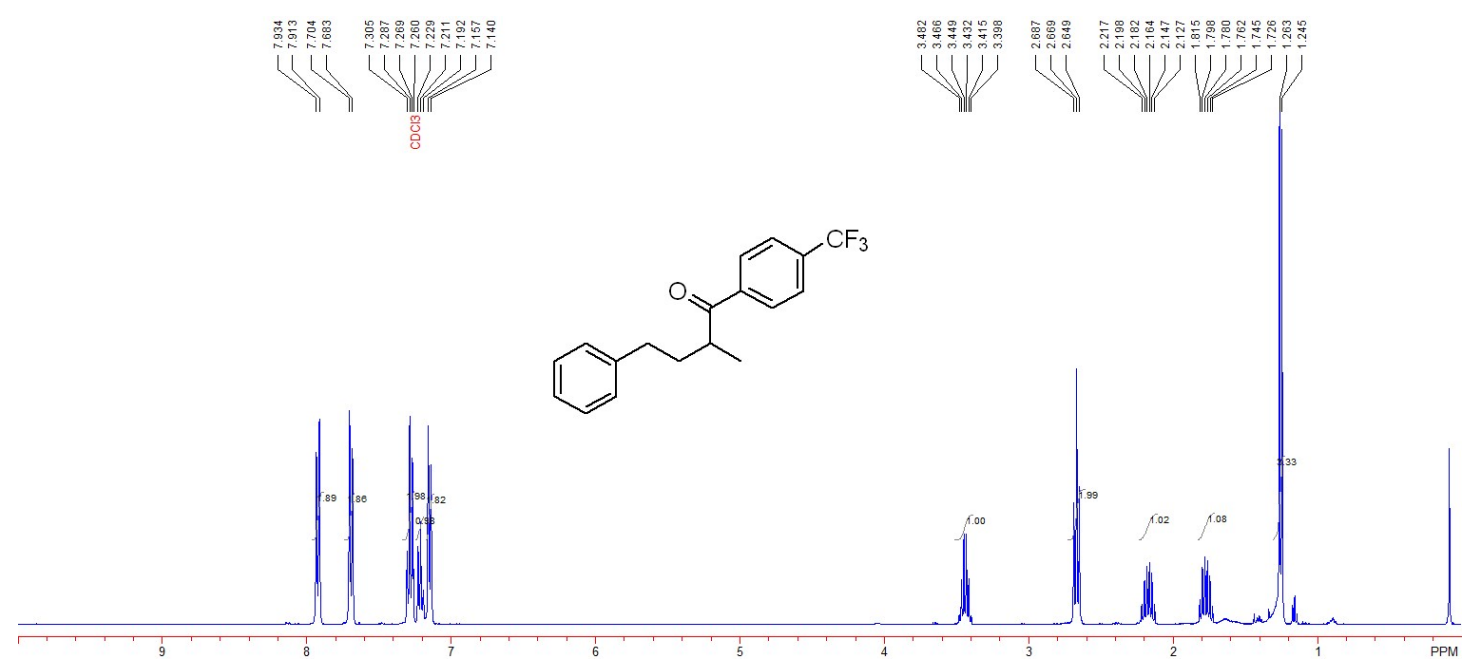

Compound $6 f{ }^{19} \mathrm{~F}$ NMR (376 MHz, $\left.\mathrm{CDCl}_{3}\right)$

$\left.\right|^{3}$
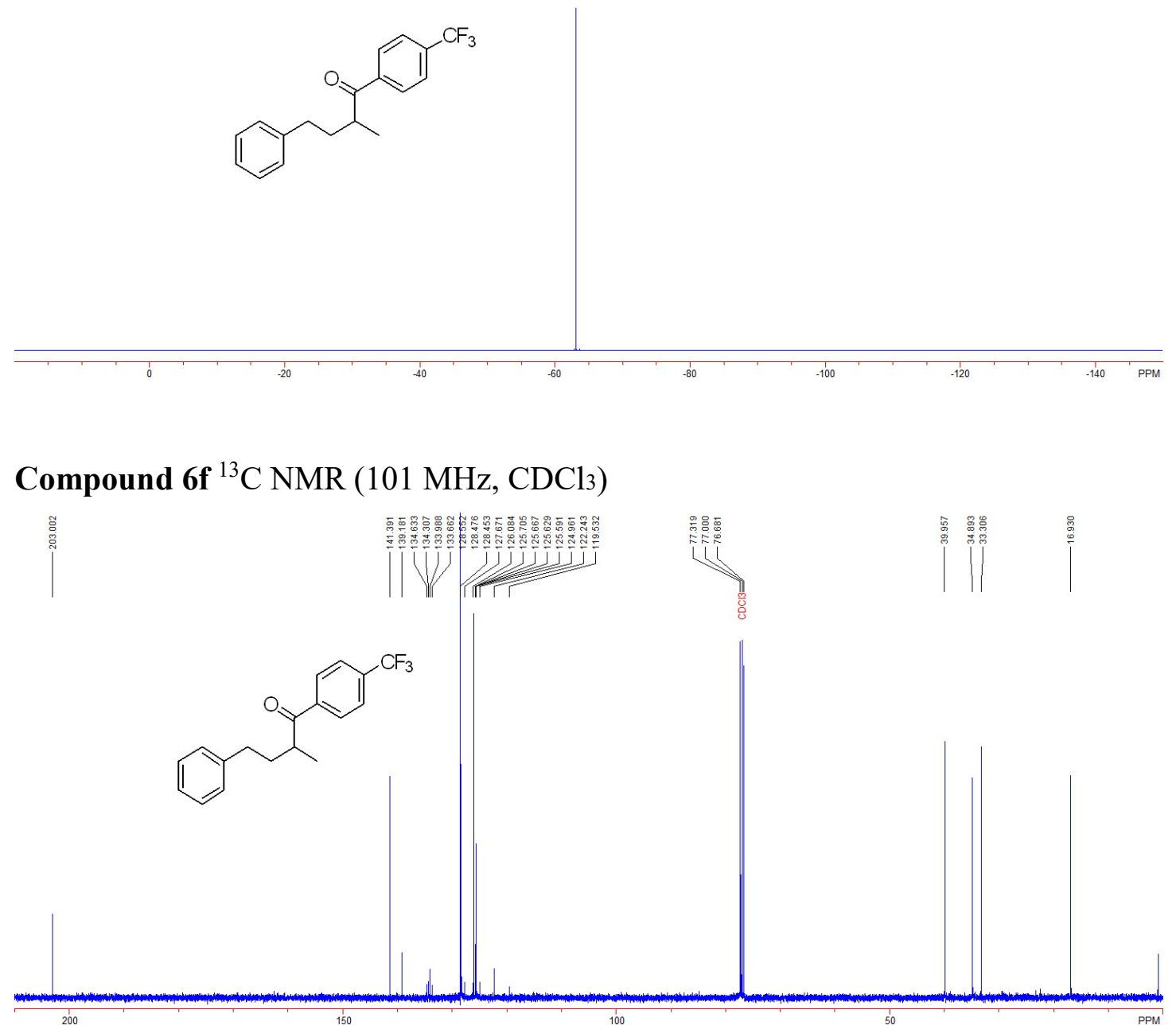

S111 


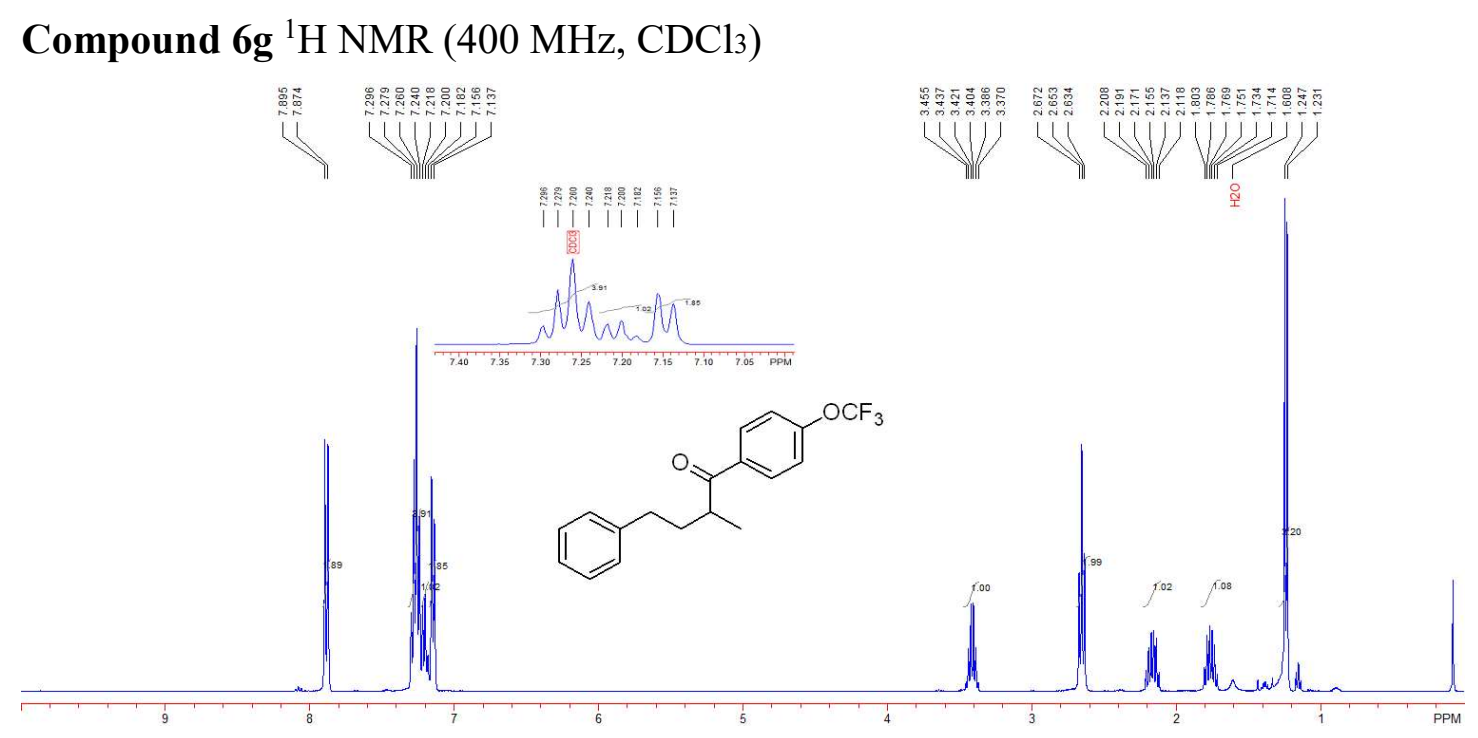

Compound 6g ${ }^{19} \mathrm{~F}$ NMR (376 MHz, $\left.\mathrm{CDCl}_{3}\right)$

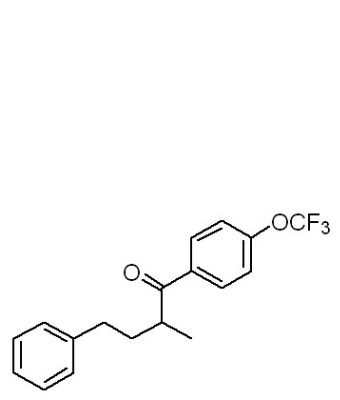

?

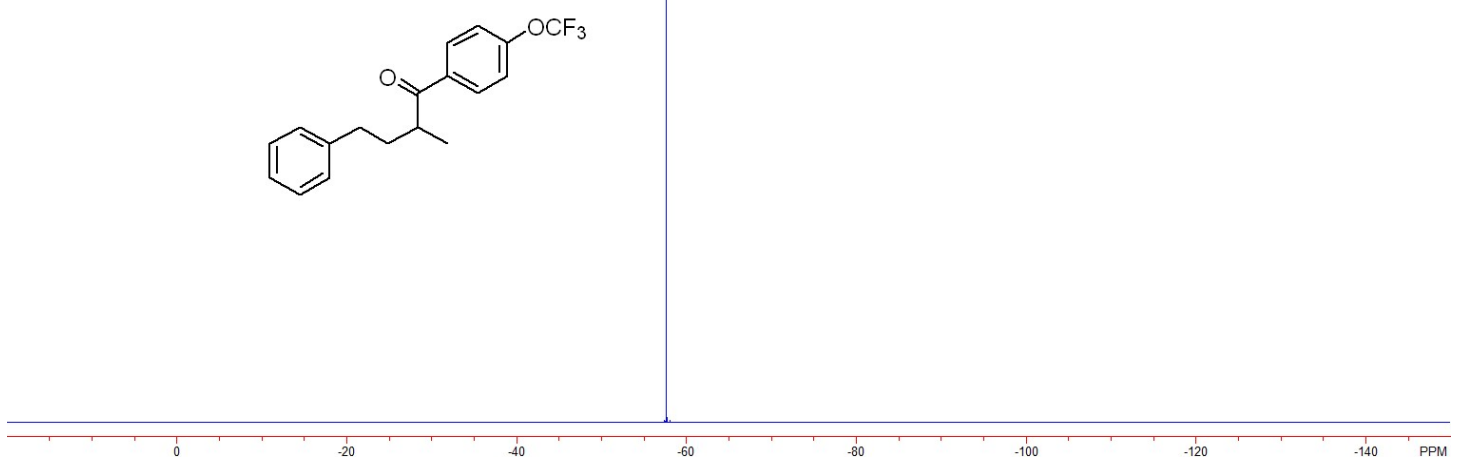

Compound mg ${ }^{13} \mathrm{C}$ NMR (101 MHz, $\left.\mathrm{CDCl}_{3}\right)$

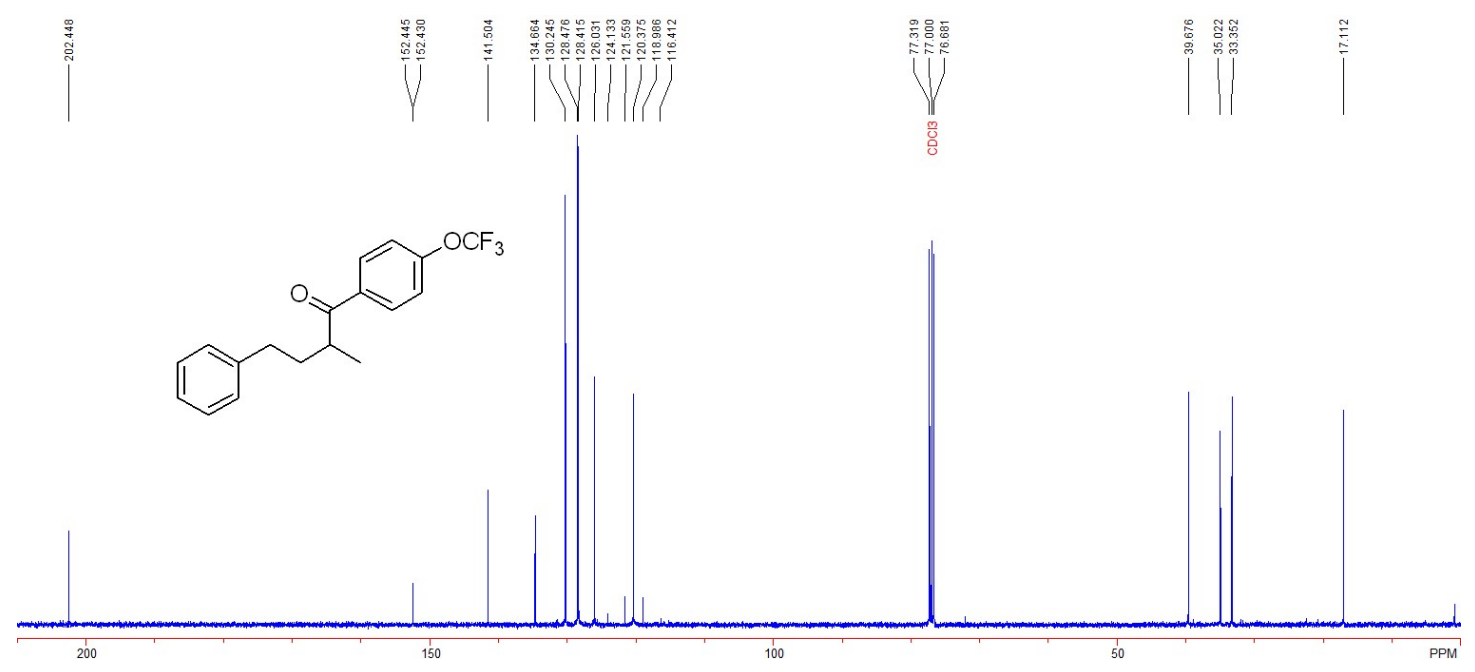

S112 


\section{Compound 6h}

${ }^{1} \mathrm{H} \mathrm{NMR}\left(400 \mathrm{MHz}, \mathrm{CDCl}_{3}\right)$
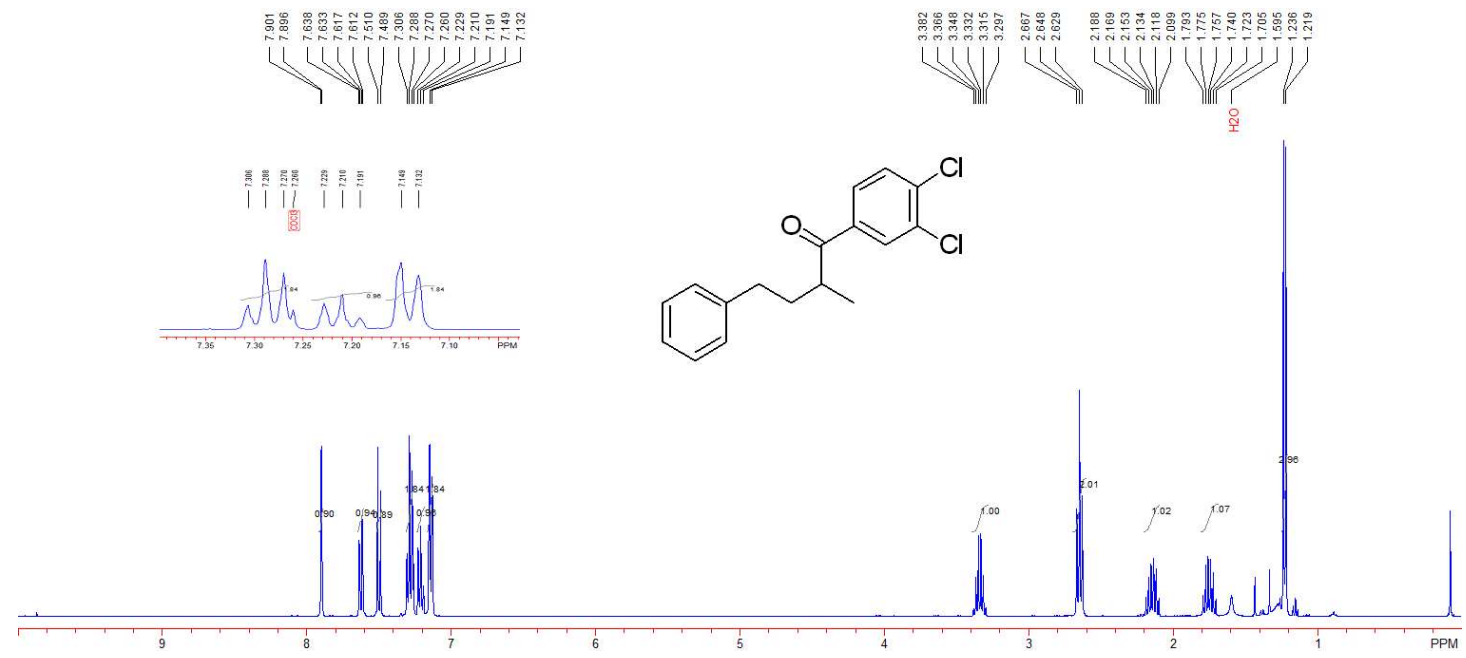

\section{Compound 6h}

${ }^{13} \mathrm{C} \mathrm{NMR}\left(101 \mathrm{MHz}, \mathrm{CDCl}_{3}\right)$

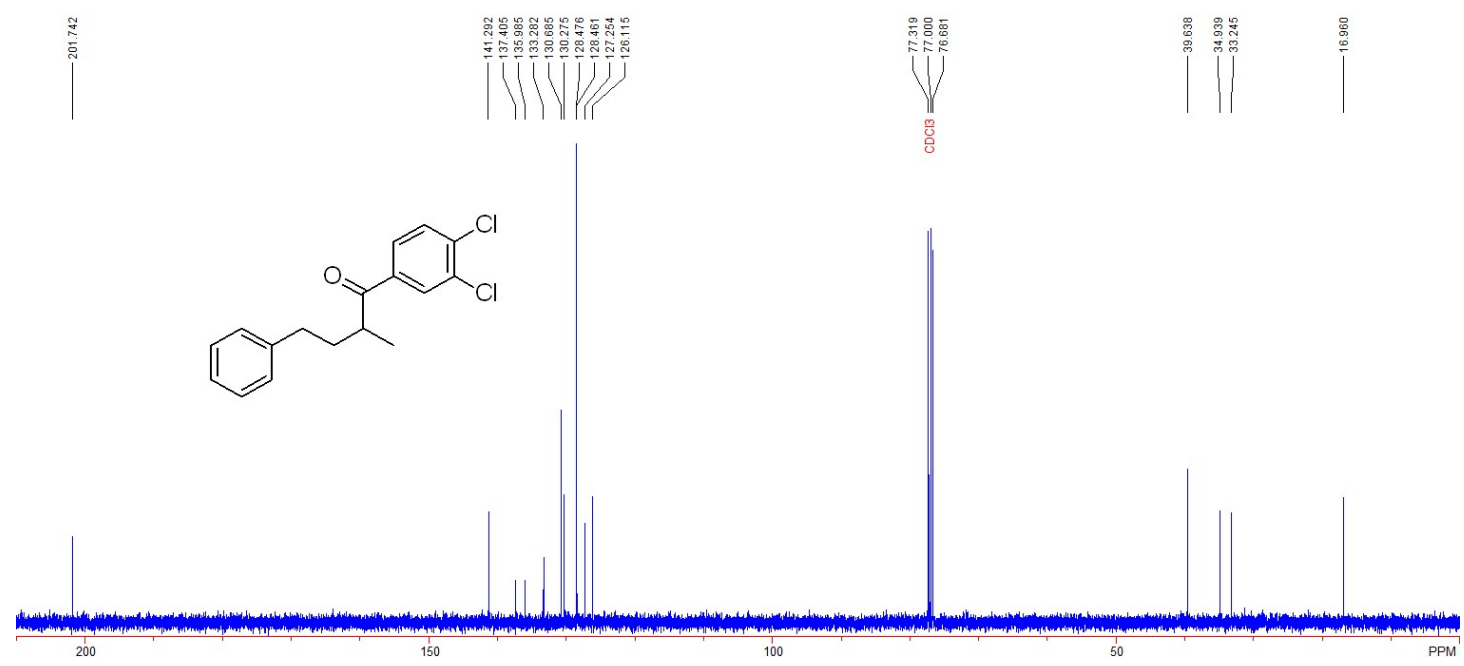




\section{Compound 6i}

${ }^{1} \mathrm{H} \mathrm{NMR}\left(400 \mathrm{MHz}, \mathrm{CDCl}_{3}\right)$

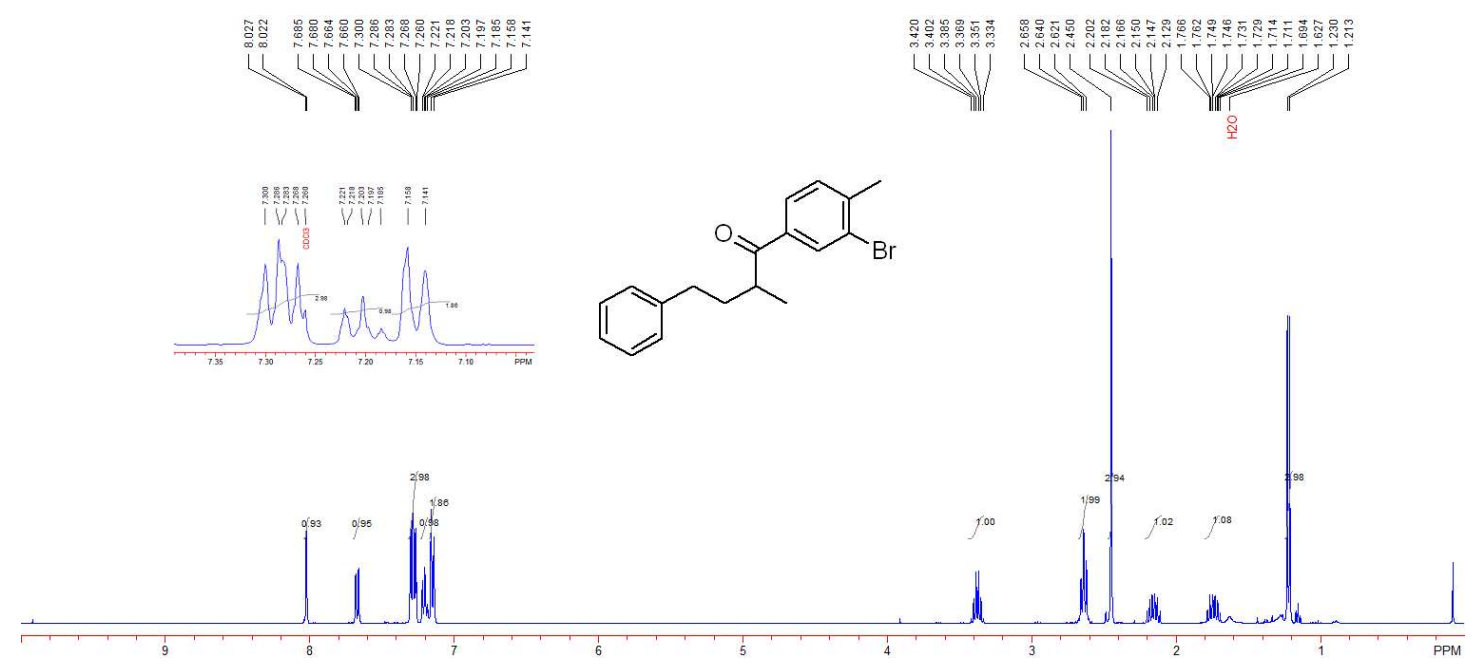

\section{Compound 6i}

${ }^{13} \mathrm{C} \mathrm{NMR}\left(101 \mathrm{MHz}, \mathrm{CDCl}_{3}\right)$
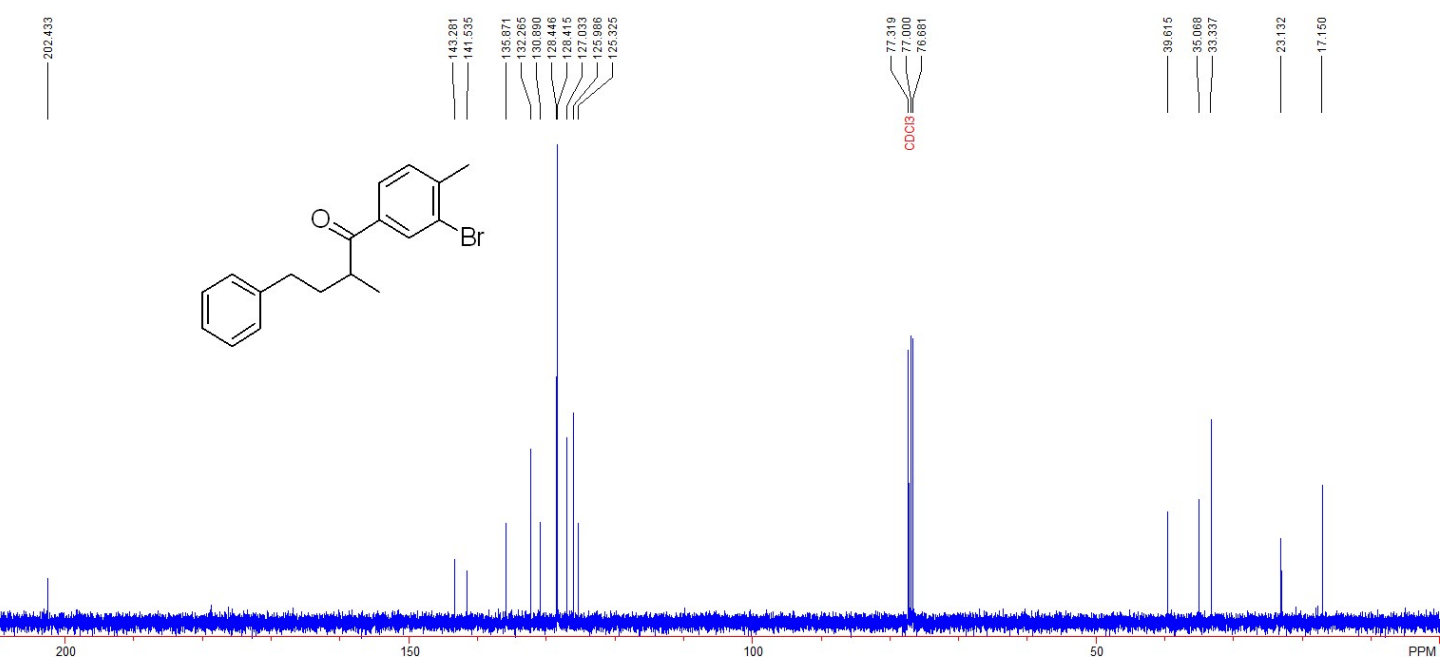
Compound oj

${ }^{1} \mathrm{H}$ NMR (400 MHz, $\mathrm{CDCl}_{3}$ )

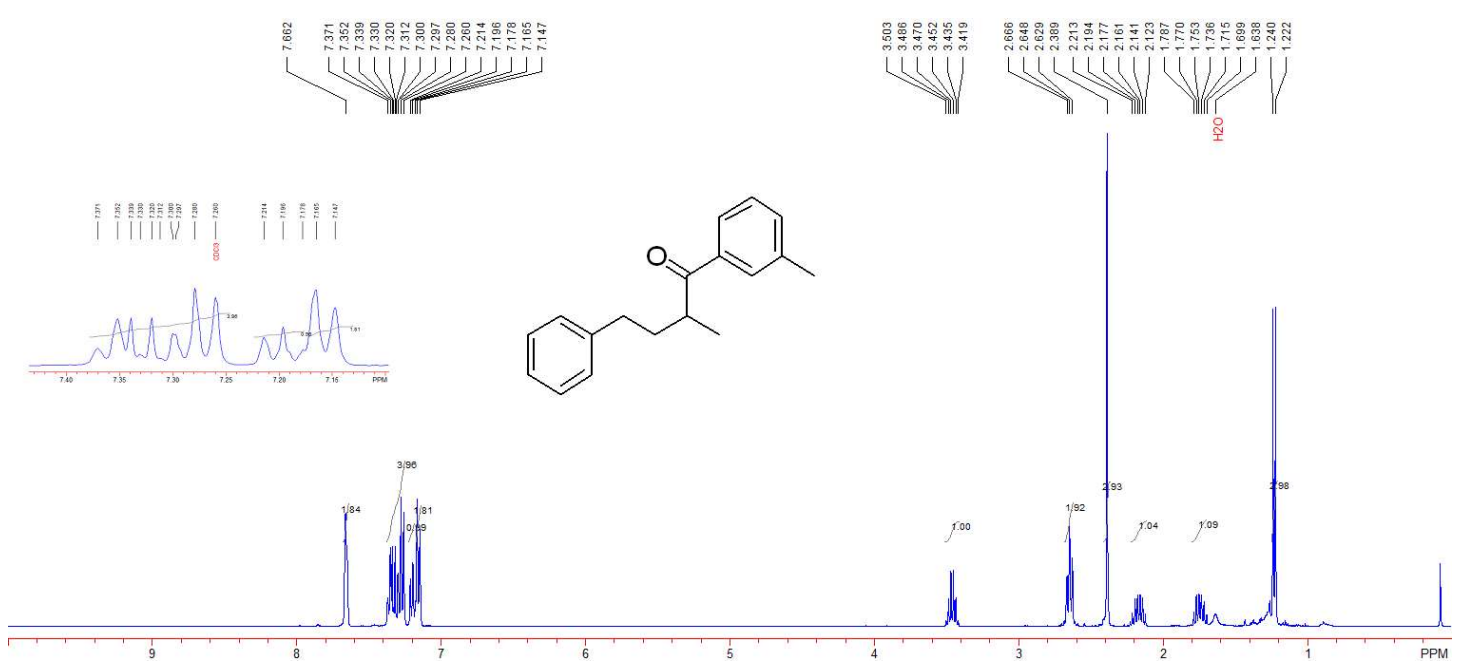

Compound oj

${ }^{13} \mathrm{C} \mathrm{NMR}\left(101 \mathrm{MHz}, \mathrm{CDCl}_{3}\right)$

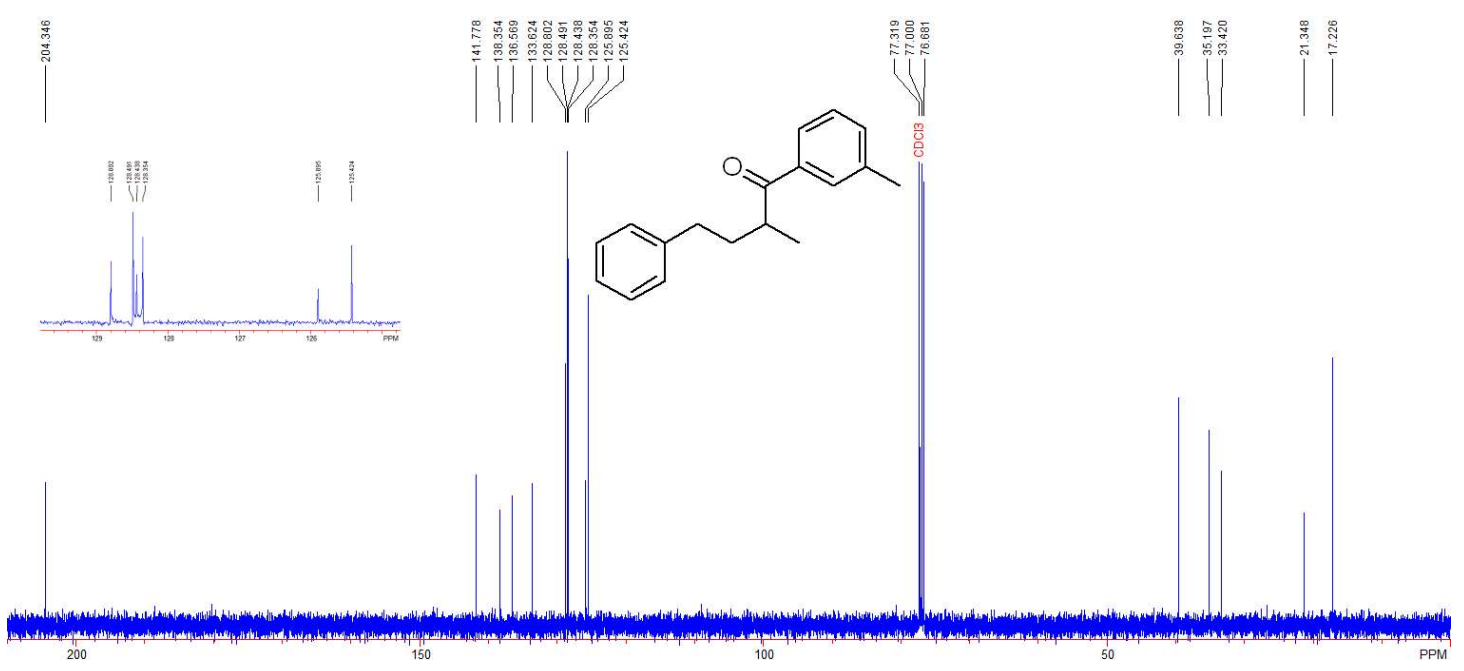

S115 


\section{Compound 6k}

${ }^{1} \mathrm{H}$ NMR (400 MHz, $\mathrm{CDCl}_{3}$ )

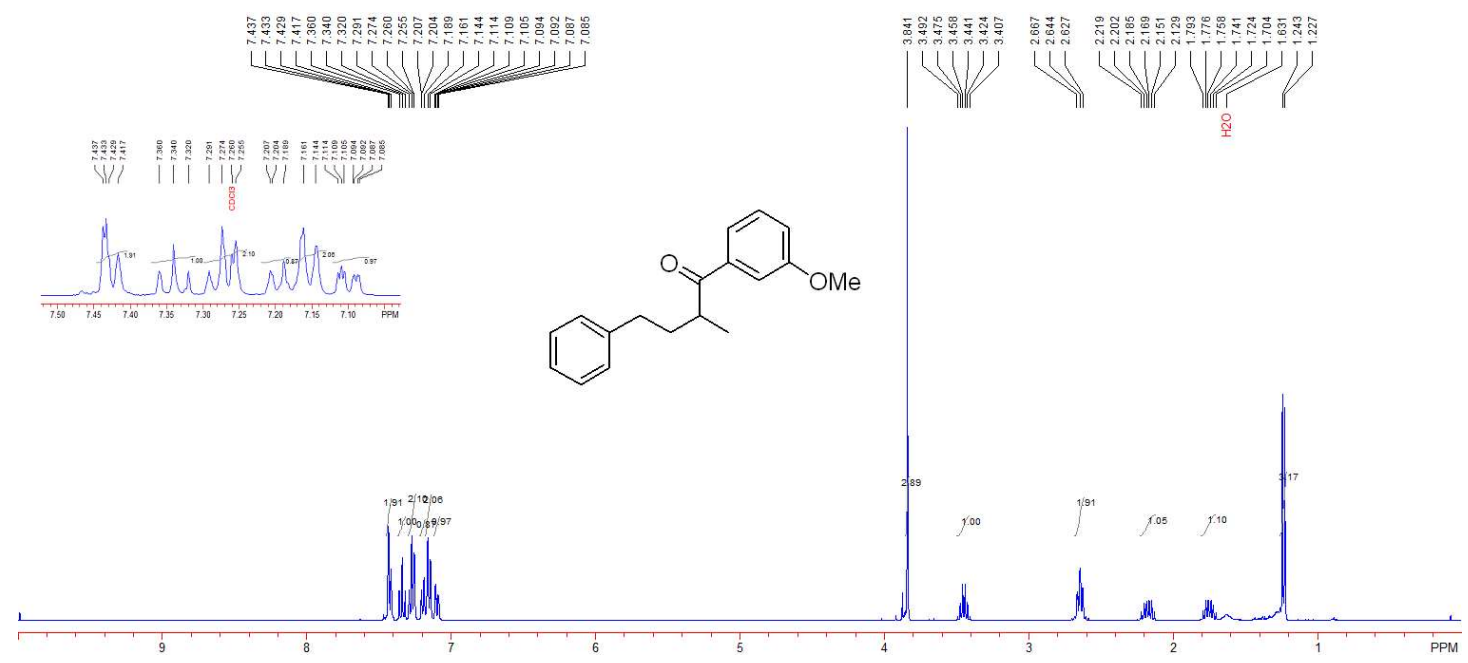

\section{Compound 6k}

${ }^{13} \mathrm{C} \mathrm{NMR}\left(101 \mathrm{MHz}, \mathrm{CDCl}_{3}\right)$

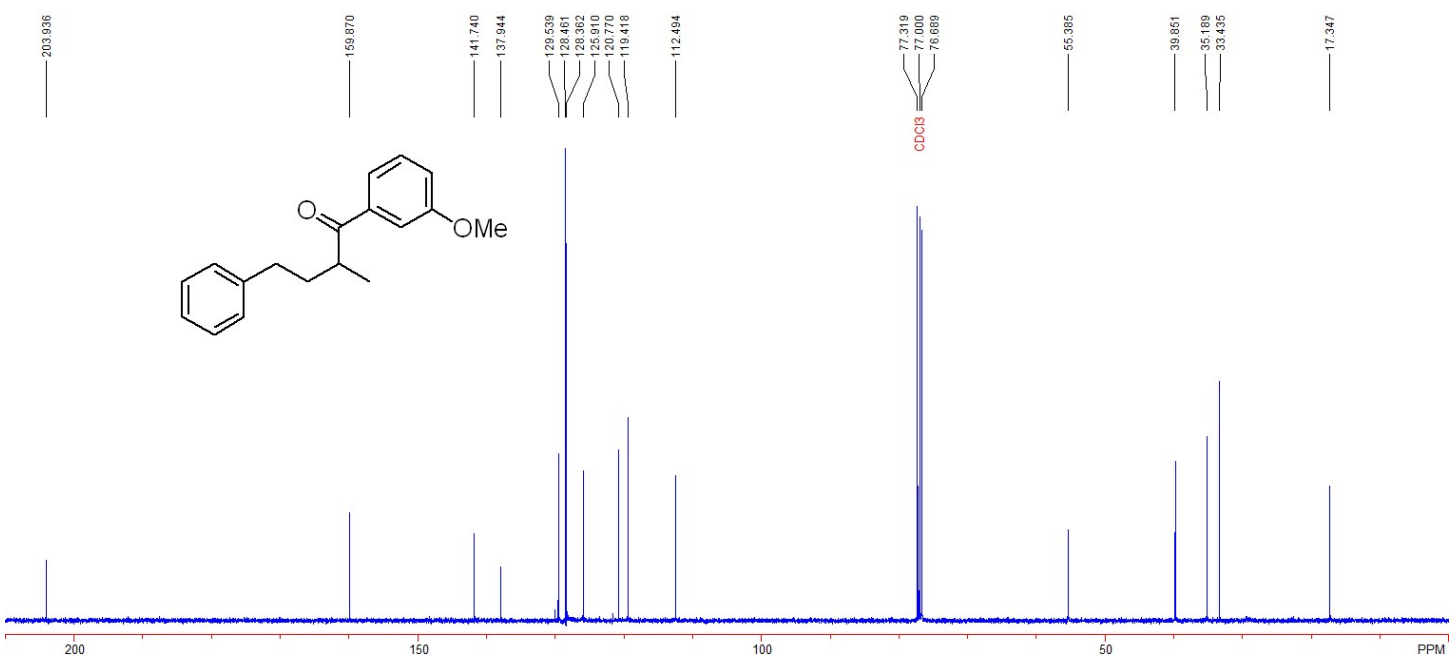




\section{Compound 61}

${ }^{1} \mathrm{H}$ NMR (400 MHz, $\mathrm{CDCl}_{3}$ )

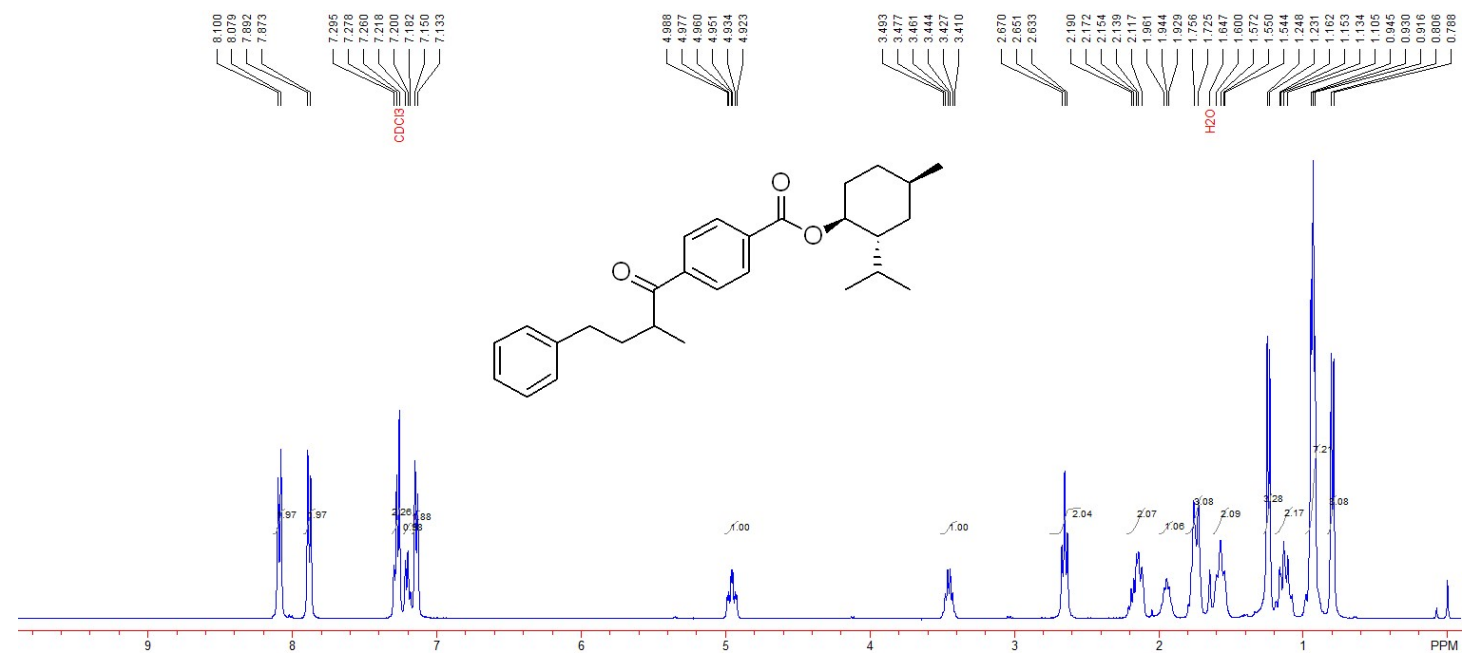

\section{Compound 61}

${ }^{13} \mathrm{C} \mathrm{NMR}\left(101 \mathrm{MHz}, \mathrm{CDCl}_{3}\right)$

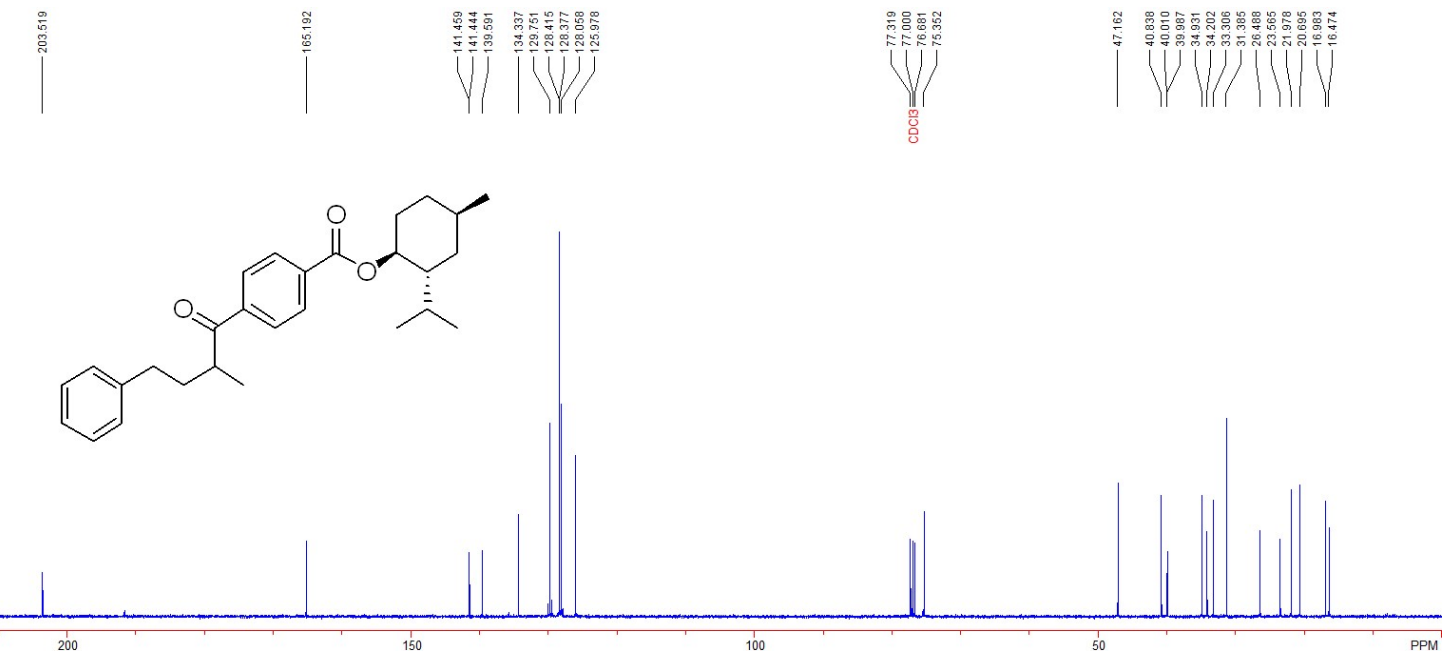




\section{Compound 6m}

${ }^{1} \mathrm{H}$ NMR (400 MHz, $\left.\mathrm{CDCl}_{3}\right)$

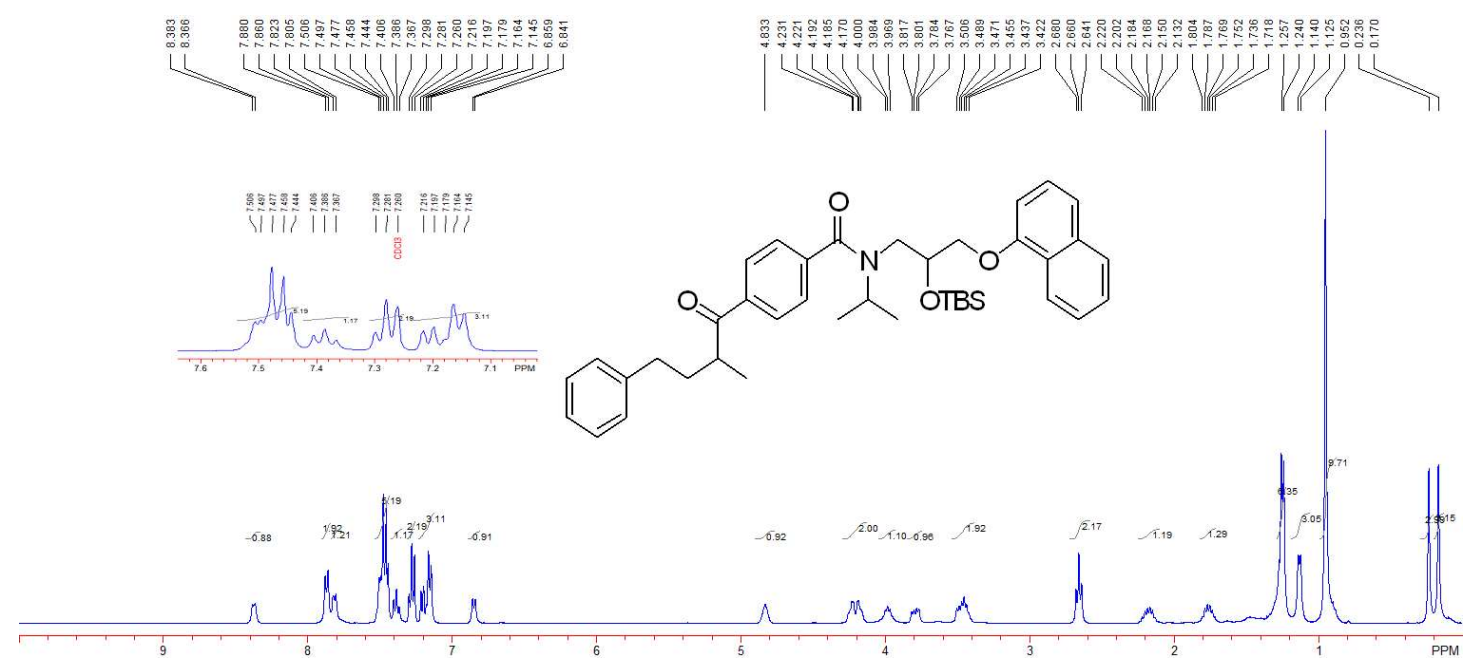

\section{Compound 6m}

${ }^{13} \mathrm{C} \mathrm{NMR}\left(101 \mathrm{MHz}, \mathrm{CDCl}_{3}\right)$

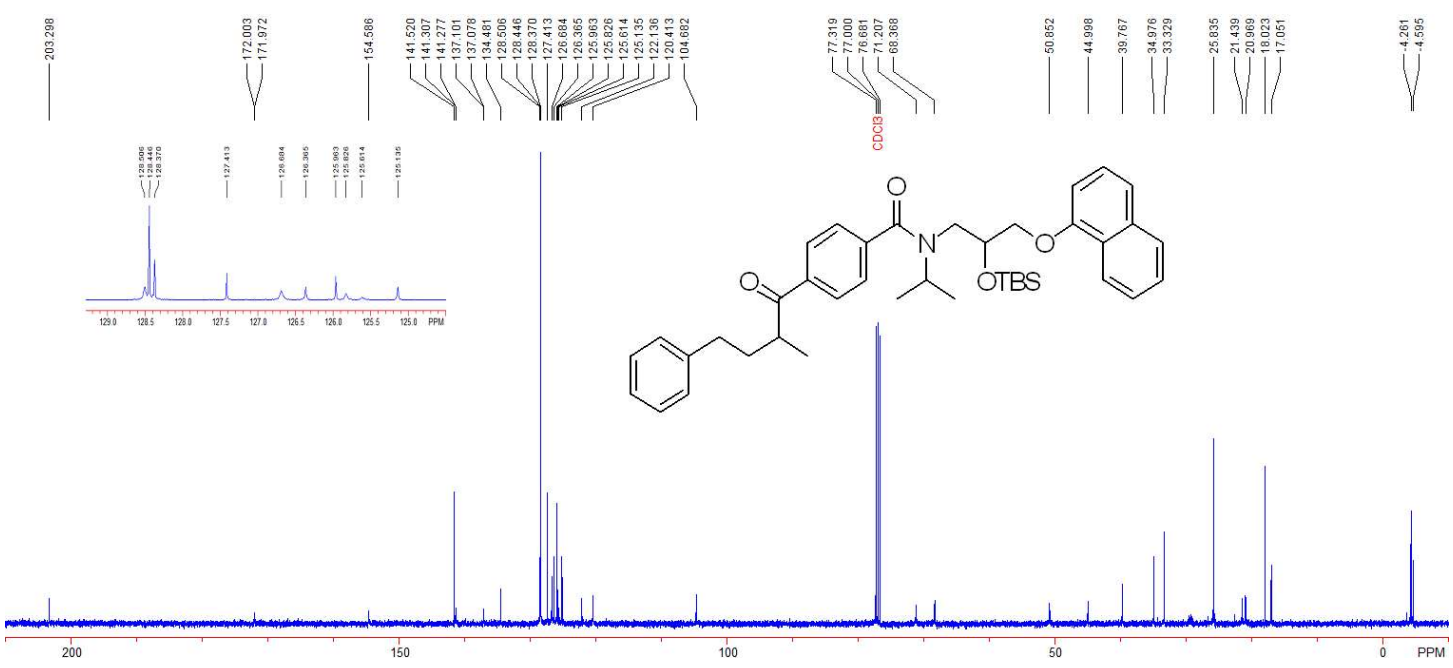




\section{Compound 6n}

${ }^{1} \mathrm{H} \mathrm{NMR}\left(400 \mathrm{MHz}, \mathrm{CDCl}_{3}\right)$

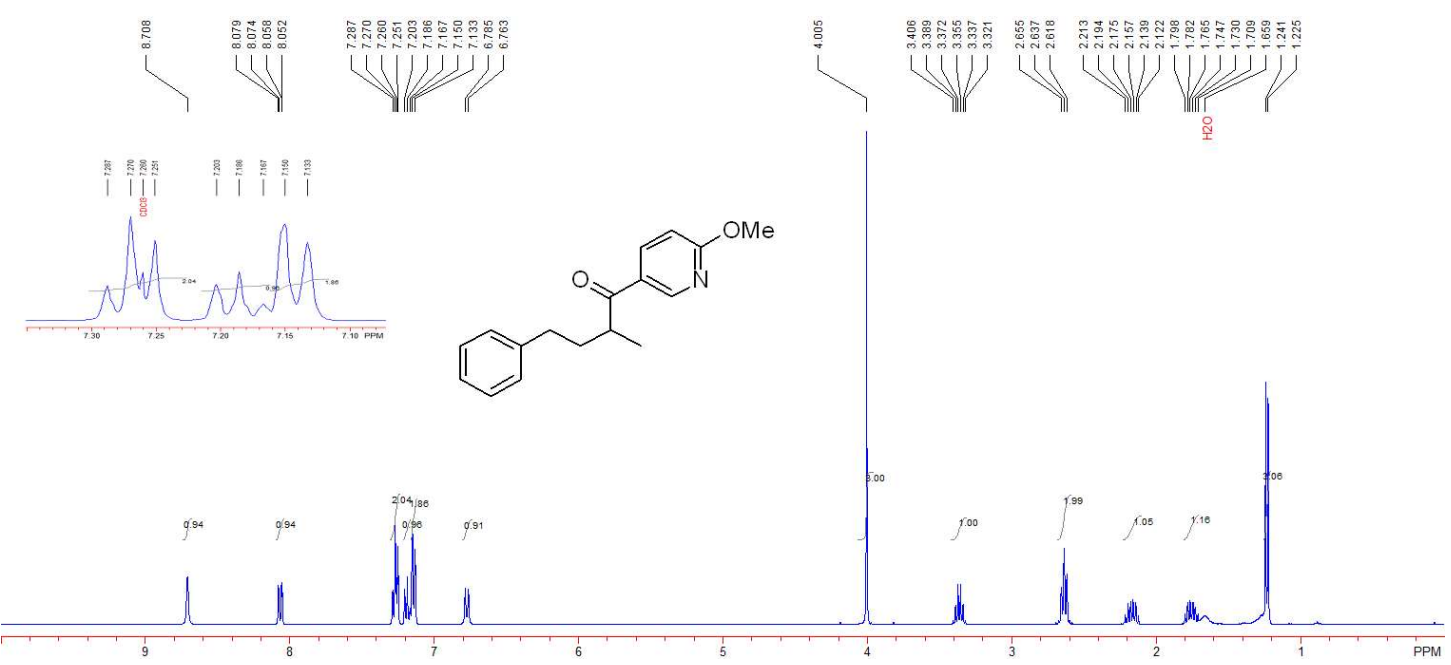

\section{Compound 6n}

${ }^{13} \mathrm{C} \mathrm{NMR}\left(101 \mathrm{MHz}, \mathrm{CDCl}_{3}\right)$

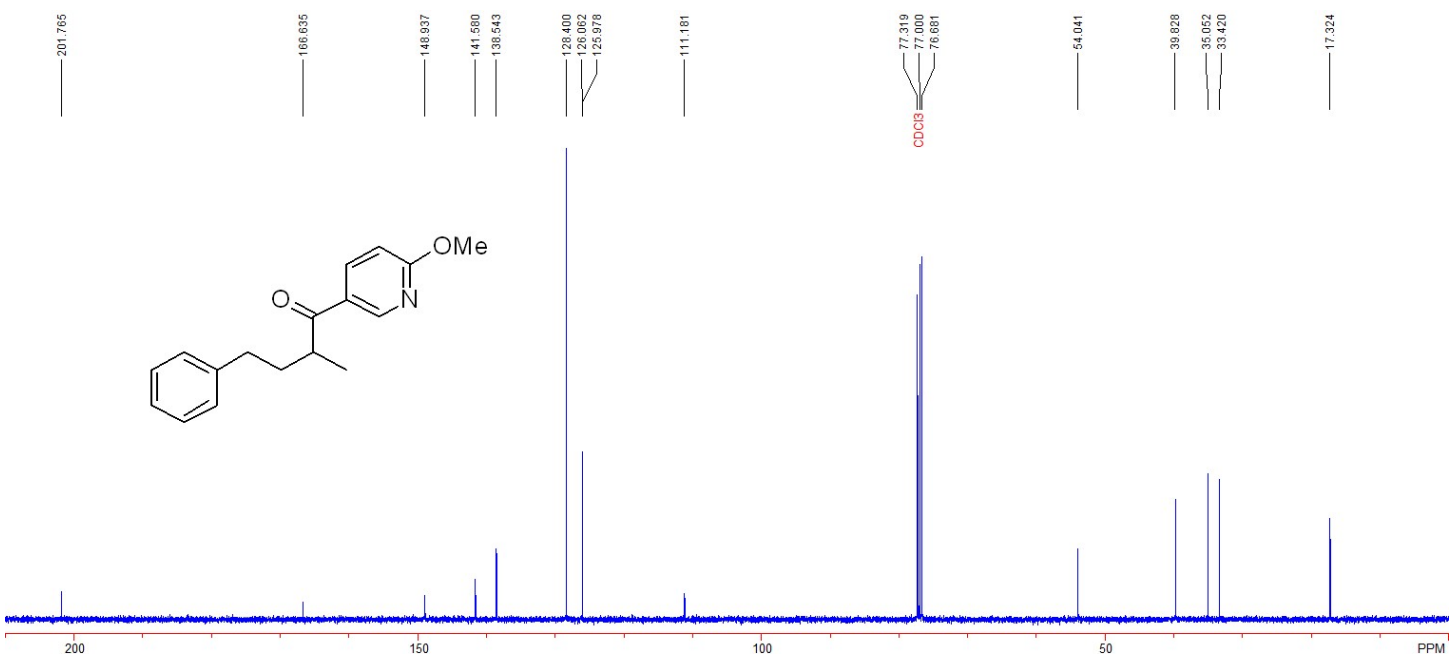




\section{Compound 6o}

${ }^{1} \mathrm{H}$ NMR (400 MHz, $\mathrm{CDCl}_{3}$ )

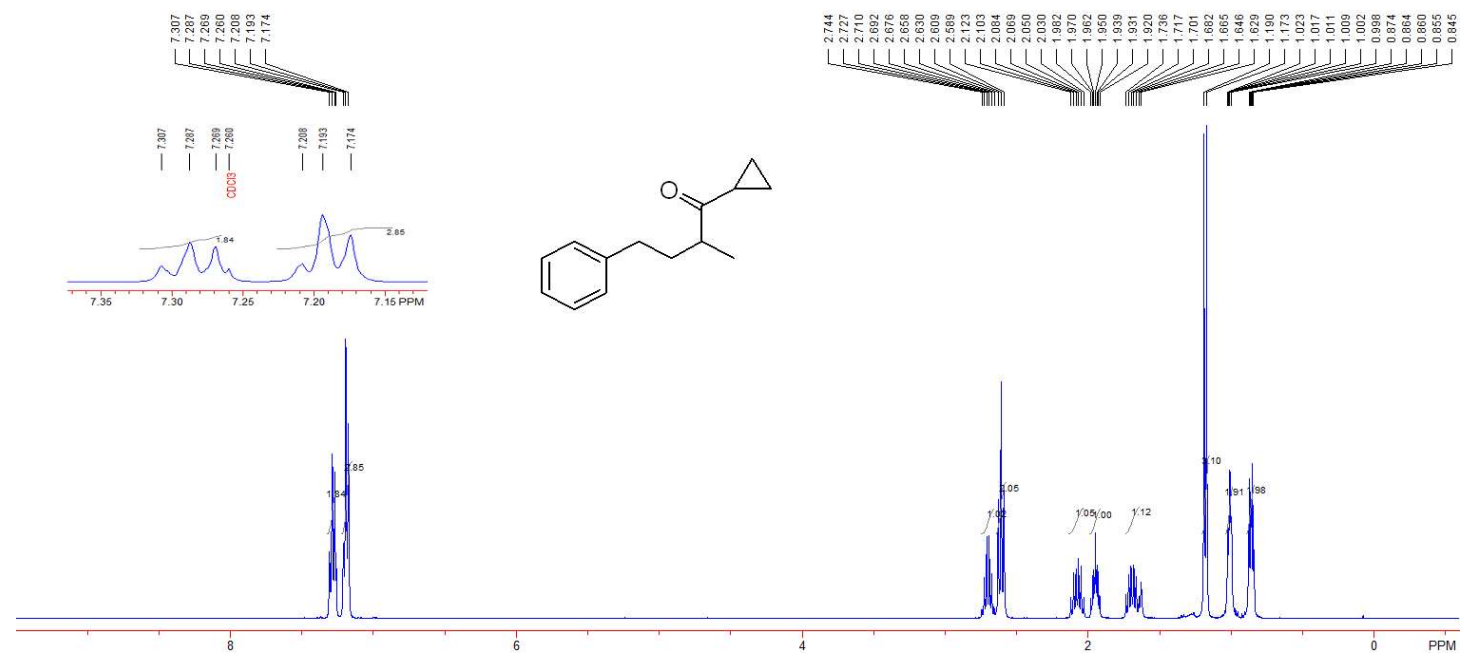

\section{Compound 60}

${ }^{13} \mathrm{C} \mathrm{NMR}\left(101 \mathrm{MHz}, \mathrm{CDCl}_{3}\right)$

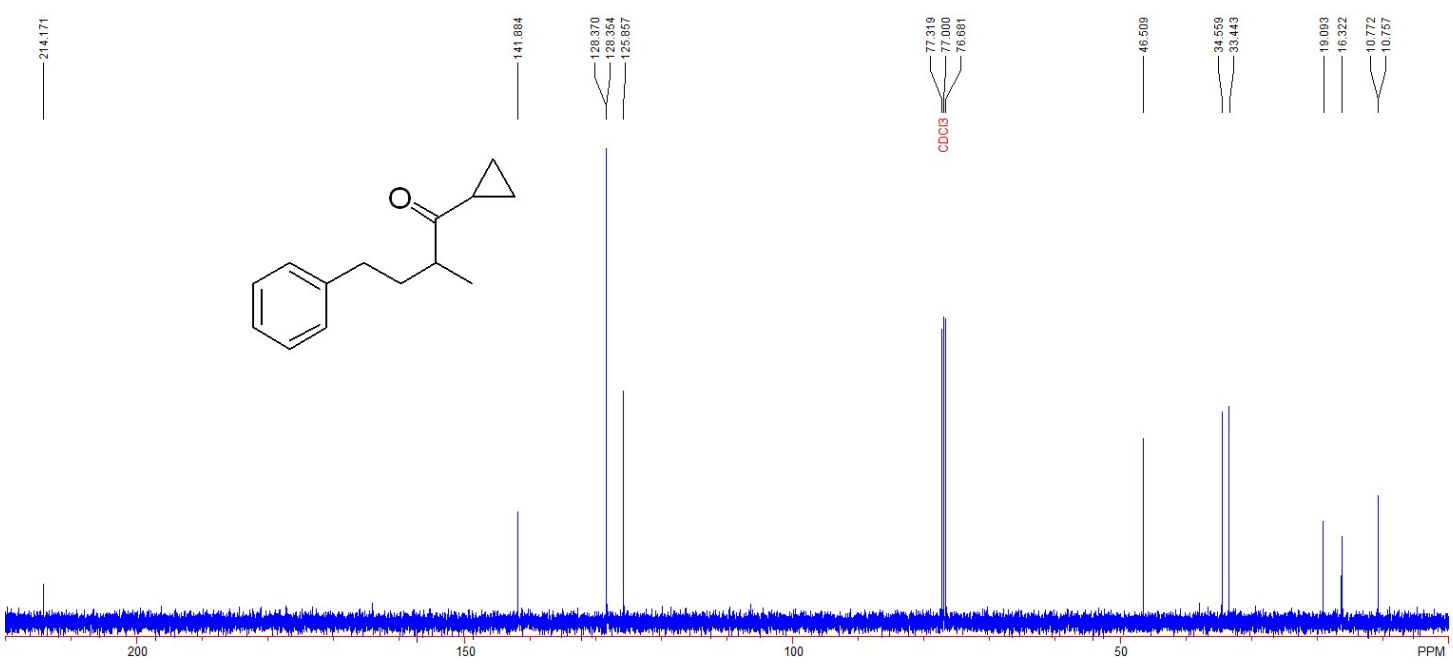




\section{Compound 8}

${ }^{1} \mathrm{H} \mathrm{NMR}\left(400 \mathrm{MHz}, \mathrm{CDCl}_{3}\right)$

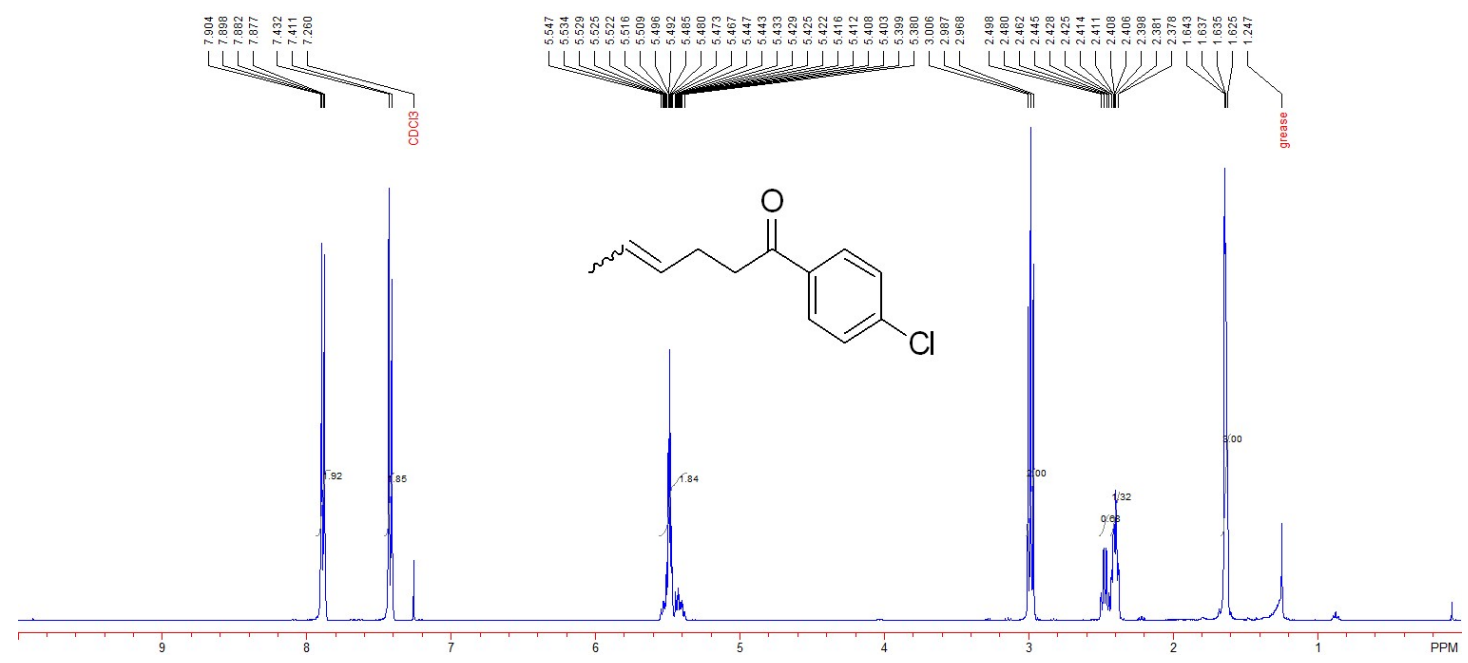

\section{Compound 8}

${ }^{13} \mathrm{C} \mathrm{NMR}\left(101 \mathrm{MHz}, \mathrm{CDCl}_{3}\right)$
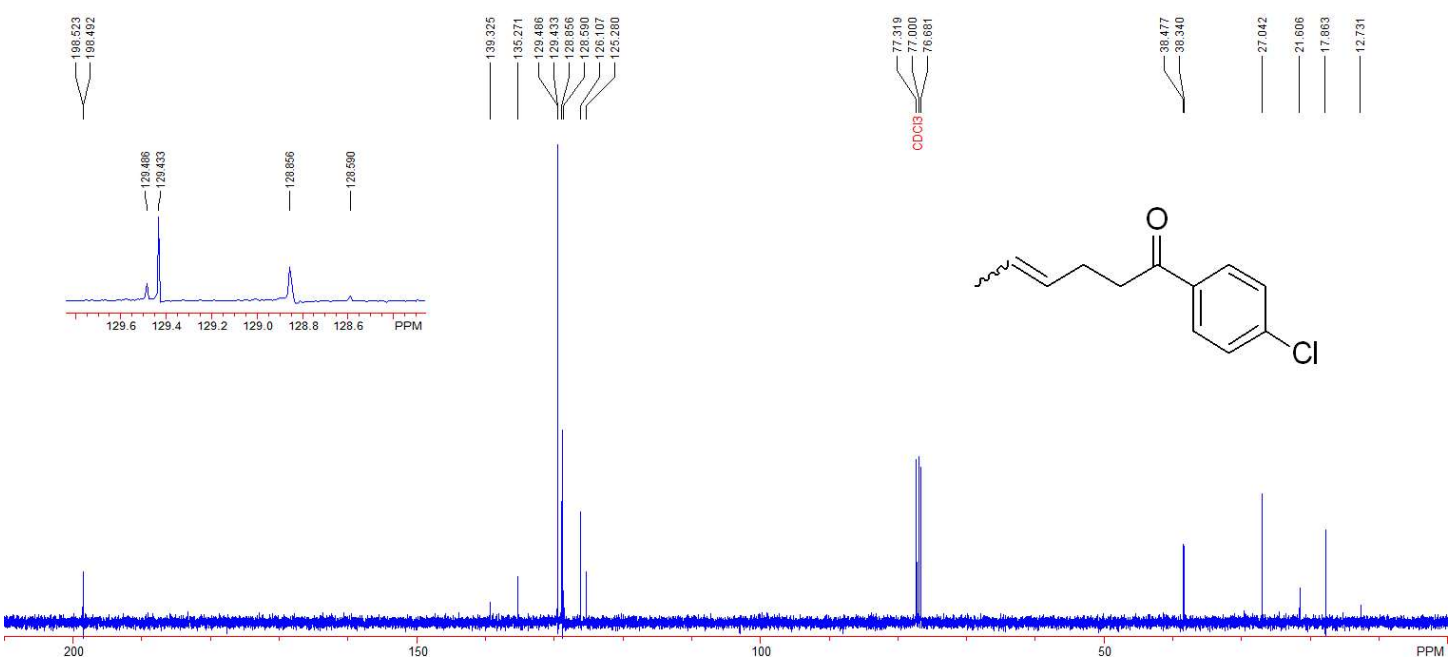


\section{Compound 10}

${ }^{1} \mathrm{H}$ NMR (400 MHz, $\mathrm{CDCl}_{3}$ )

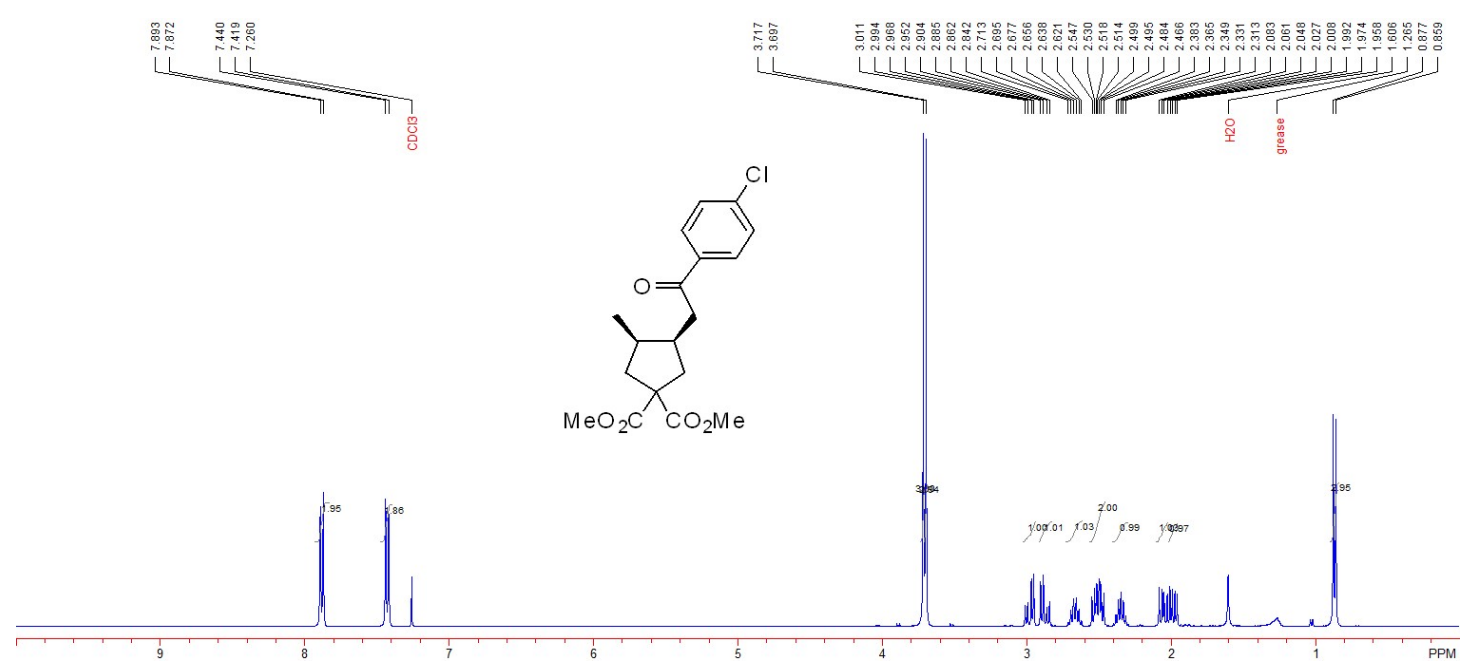

\section{Compound 10}

${ }^{13} \mathrm{C} \mathrm{NMR}\left(101 \mathrm{MHz}, \mathrm{CDCl}_{3}\right)$

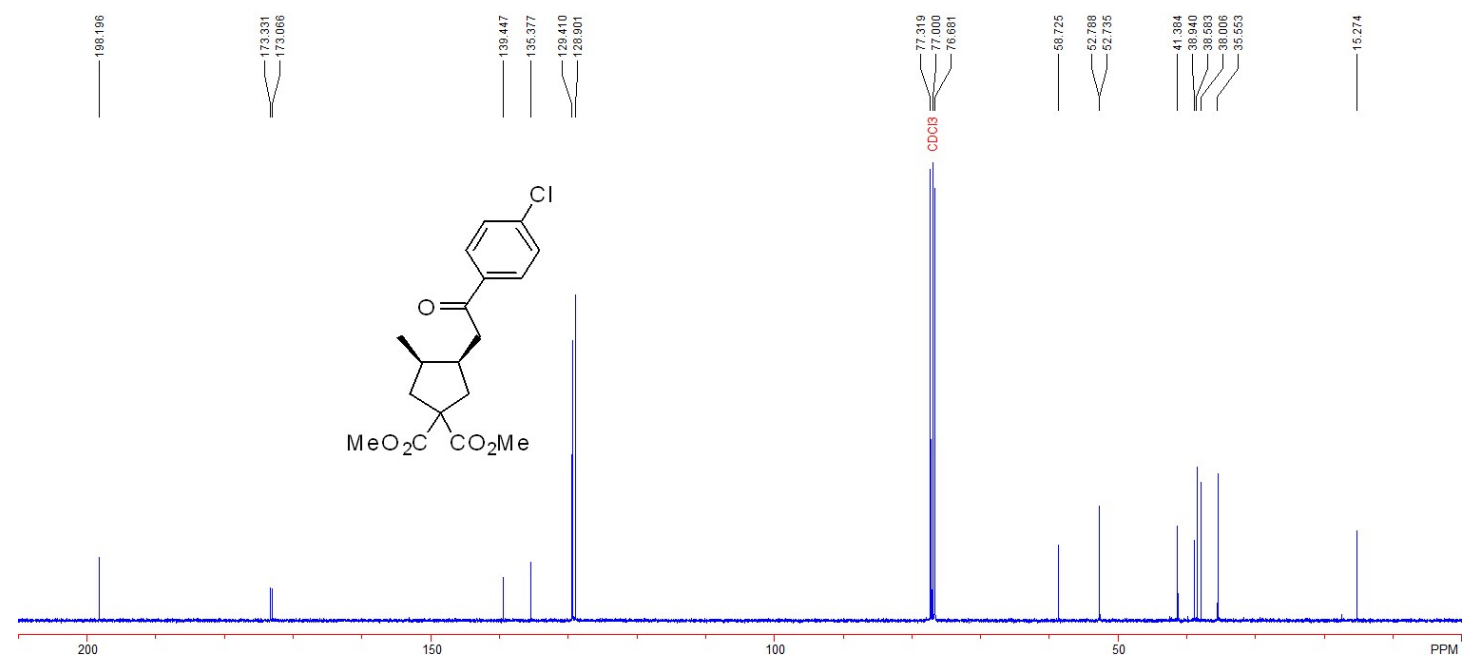




\section{2D NMR Spectra of 10}

\section{COSY spectrum of 10}
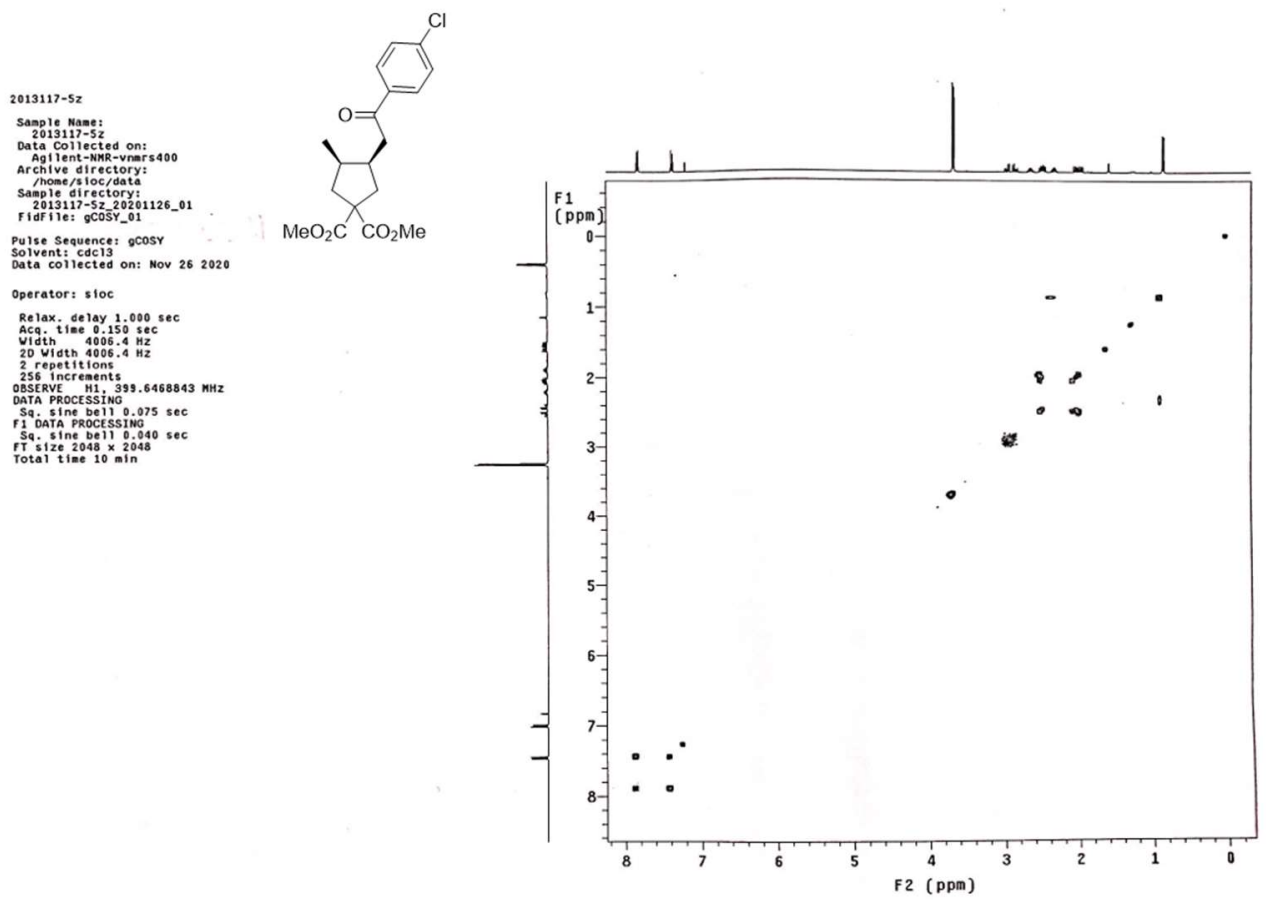

HSQC spectrum of 10

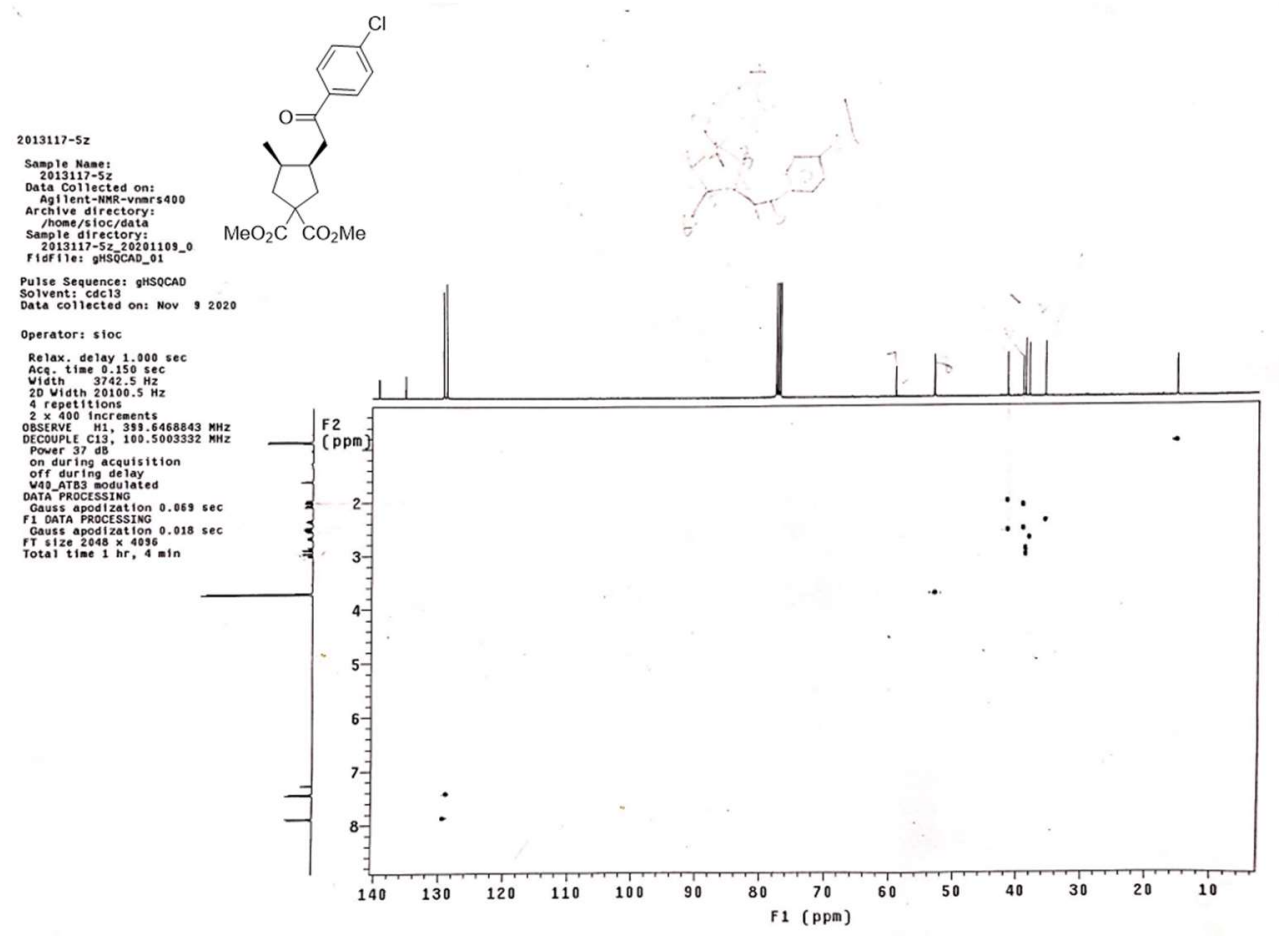




\section{NOE spectrum of 10}
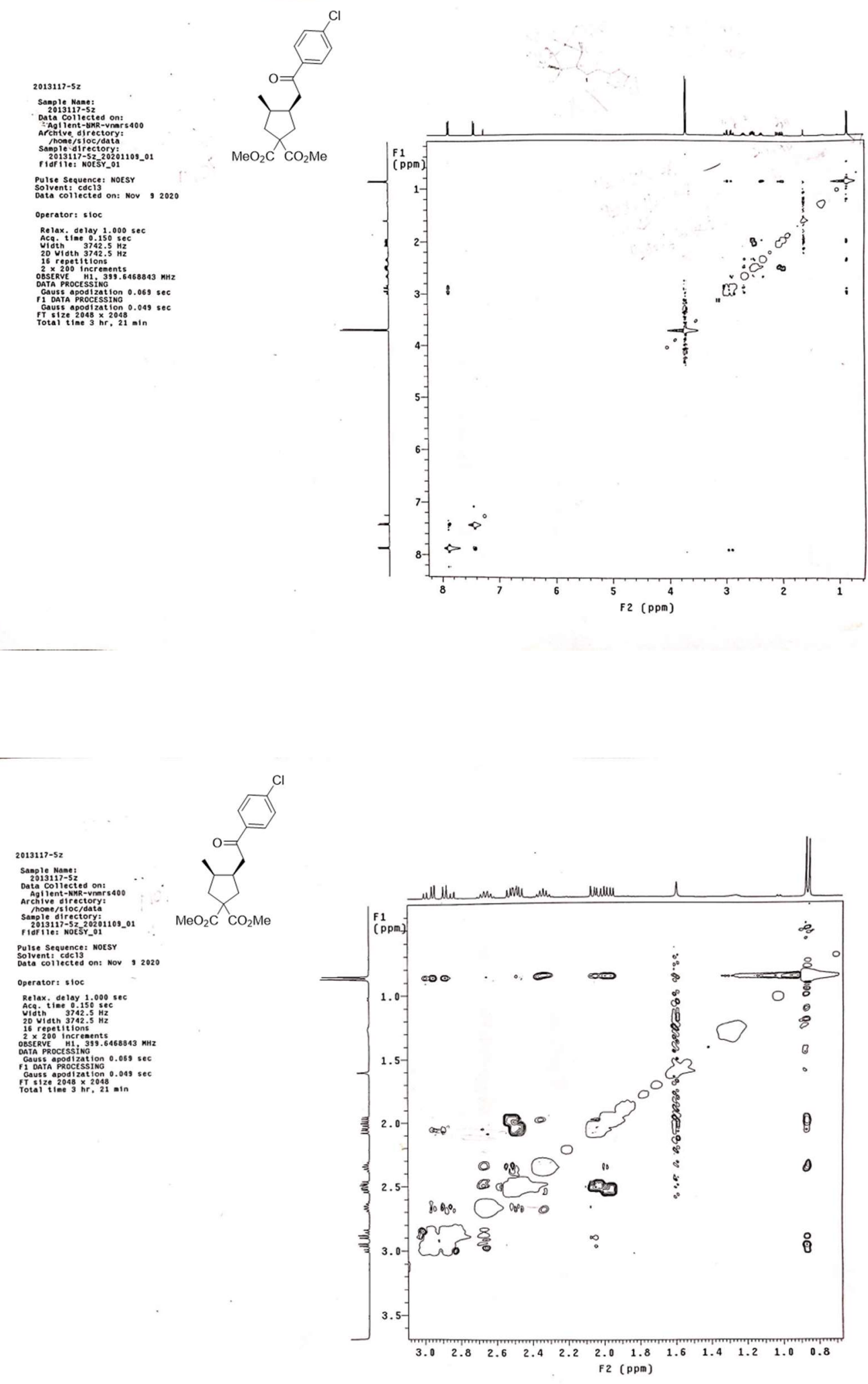
8. X-ray crystallographic data of compounds $3 a$ and $3 b$

\section{Crystal Structure of 3a}

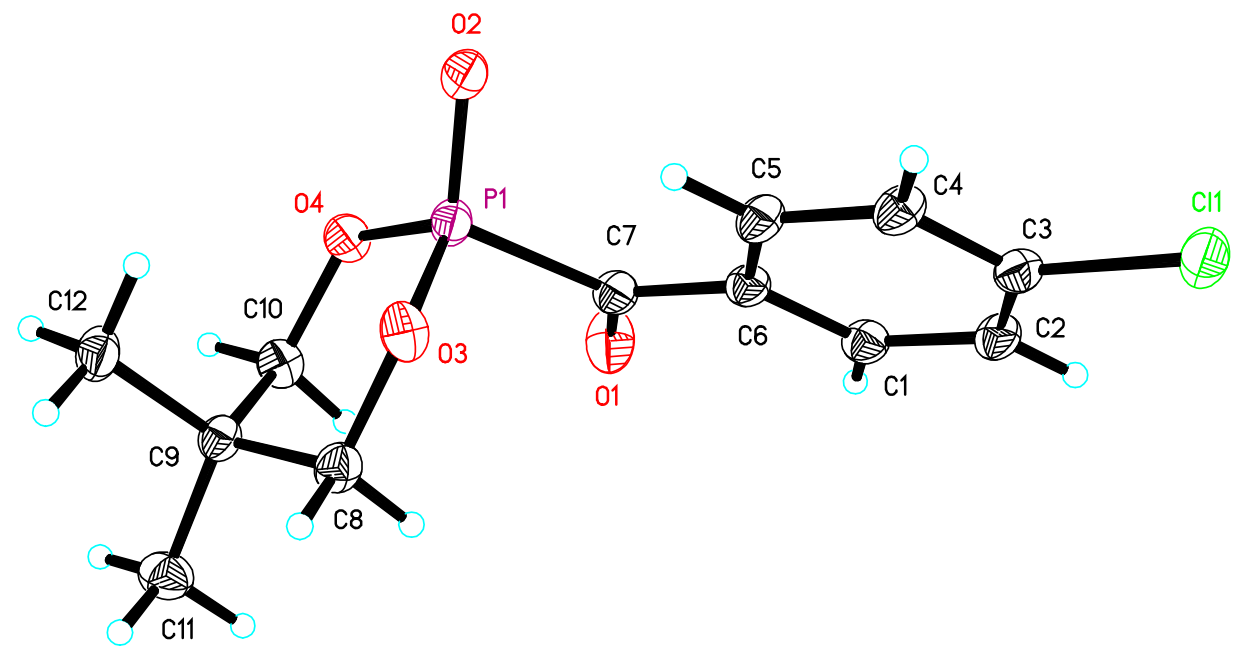

Table S4. Crystal data and structure refinement for mo_d8v21054_0m.

Identification code

Empirical formula

Formula weight

Temperature

Wavelength

Crystal system

Space group

Unit cell dimensions

Volume

Z

Density (calculated)

Absorption coefficient

$\mathrm{F}(000)$

Crystal size

Theta range for data collection

Index ranges

Reflections collected

Independent reflections mo_d8v21054_0m

$\mathrm{C} 12 \mathrm{H} 14 \mathrm{Cl} \mathrm{O} 4 \mathrm{P}$

288.65

193(2) K

$0.71073 \AA$

Monoclinic

P $21 /$ n

$\mathrm{a}=6.8587(6) \AA$

$\alpha=90^{\circ}$.

$\mathrm{b}=10.5793(9) \AA$

$\beta=90.880(3)^{\circ}$.

$\mathrm{c}=18.3384(15) \AA$ $\gamma=90^{\circ}$.

$1330.5(2) \AA^{3}$

4

$1.441 \mathrm{Mg} / \mathrm{m}^{3}$

$0.410 \mathrm{~mm}^{-1}$

600

$0.200 \times 0.150 \times 0.120 \mathrm{~mm}^{3}$

2.221 to $25.998^{\circ}$.

$-8<=\mathrm{h}<=8,-13<=\mathrm{k}<=13,-22<=\mathrm{l}<=22$

11884

$2590[\mathrm{R}(\mathrm{int})=0.0441]$ 
Completeness to theta $=25.242^{\circ}$

Absorption correction

Max. and min. transmission

Refinement method

Data / restraints / parameters

Goodness-of-fit on $\mathrm{F}^{2}$

Final $\mathrm{R}$ indices [I $>2 \operatorname{sigma}(\mathrm{I})]$

$\mathrm{R}$ indices (all data)

Extinction coefficient

Largest diff. peak and hole
$98.8 \%$

Semi-empirical from equivalents

0.7456 and 0.6035

Full-matrix least-squares on $\mathrm{F}^{2}$

$2590 / 0$ / 166

1.127

$\mathrm{R} 1=0.0522, \mathrm{wR} 2=0.1470$

$\mathrm{R} 1=0.0557, \mathrm{wR} 2=0.1496$

$0.032(6)$

0.649 and -0.265 e. $\AA^{-3}$

Table S5. Atomic coordinates (x 104) and equivalent isotropic displacement parameters $\left(\AA^{2} \mathrm{x}\right.$ $10^{3}$ ) for mo_d8v21054_0m. U(eq) is defined as one third of the trace of the orthogonalized $U^{i j}$ tensor.

\begin{tabular}{|c|c|c|c|c|}
\hline & $\mathrm{x}$ & $\mathrm{y}$ & $\mathrm{z}$ & $\mathrm{U}(\mathrm{eq})$ \\
\hline $\mathrm{Cl}(1)$ & $7782(1)$ & $2519(1)$ & $5169(1)$ & $45(1)$ \\
\hline $\mathrm{P}(1)$ & $6609(1)$ & $8562(1)$ & $6798(1)$ & $33(1)$ \\
\hline $\mathrm{O}(1)$ & 7076(4) & $8828(2)$ & $5373(1)$ & $54(1)$ \\
\hline $\mathrm{O}(2)$ & $8051(4)$ & $8031(2)$ & $7308(1)$ & $49(1)$ \\
\hline $\mathrm{O}(3)$ & $4456(3)$ & $8166(2)$ & $6960(1)$ & $39(1)$ \\
\hline $\mathrm{O}(4)$ & $6652(3)$ & $10036(2)$ & $6784(1)$ & $38(1)$ \\
\hline $\mathrm{C}(1)$ & $7374(4)$ & $6256(3)$ & $4969(2)$ & $34(1)$ \\
\hline $\mathrm{C}(2)$ & $7554(4)$ & $4990(3)$ & $4804(2)$ & $35(1)$ \\
\hline $\mathrm{C}(3)$ & $7515(4)$ & $4119(3)$ & $5365(2)$ & $34(1)$ \\
\hline $\mathrm{C}(4)$ & $7278(5)$ & $4471(3)$ & $6081(2)$ & $36(1)$ \\
\hline$C(5)$ & $7098(5)$ & $5743(3)$ & $6244(2)$ & $35(1)$ \\
\hline$C(6)$ & $7152(4)$ & $6654(3)$ & $5689(2)$ & $31(1)$ \\
\hline$C(7)$ & $6992(4)$ & $8030(3)$ & $5846(2)$ & $34(1)$ \\
\hline$C(8)$ & 2833(4) & $8948(3)$ & $6681(2)$ & $36(1)$ \\
\hline $\mathrm{C}(9)$ & $3106(4)$ & 10323(3) & $6889(2)$ & $32(1)$ \\
\hline $\mathrm{C}(10)$ & $4966(5)$ & $10795(3)$ & $6555(2)$ & $37(1)$ \\
\hline $\mathrm{C}(11)$ & $1415(5)$ & $11084(4)$ & $6556(2)$ & $45(1)$ \\
\hline$C(12)$ & $3144(5)$ & $10455(3)$ & $7722(2)$ & $42(1)$ \\
\hline
\end{tabular}


Table S6. Bond lengths $[\AA]$ and angles $\left[{ }^{\circ}\right]$ for mo_d8v21054_0m.

\begin{tabular}{|c|c|}
\hline $\mathrm{Cl}(1)-\mathrm{C}(3)$ & $1.741(3)$ \\
\hline $\mathrm{P}(1)-\mathrm{O}(2)$ & $1.463(2)$ \\
\hline $\mathrm{P}(1)-\mathrm{O}(4)$ & $1.560(2)$ \\
\hline $\mathrm{P}(1)-\mathrm{O}(3)$ & $1.568(2)$ \\
\hline $\mathrm{P}(1)-\mathrm{C}(7)$ & $1.858(3)$ \\
\hline $\mathrm{O}(1)-\mathrm{C}(7)$ & $1.211(4)$ \\
\hline $\mathrm{O}(3)-\mathrm{C}(8)$ & $1.473(4)$ \\
\hline $\mathrm{O}(4)-\mathrm{C}(10)$ & $1.464(4)$ \\
\hline$C(1)-C(2)$ & $1.379(5)$ \\
\hline $\mathrm{C}(1)-\mathrm{C}(6)$ & $1.396(4)$ \\
\hline $\mathrm{C}(1)-\mathrm{H}(1)$ & 0.9500 \\
\hline$C(2)-C(3)$ & $1.381(5)$ \\
\hline $\mathrm{C}(2)-\mathrm{H}(2)$ & 0.9500 \\
\hline$C(3)-C(4)$ & $1.377(4)$ \\
\hline$C(4)-C(5)$ & $1.384(4)$ \\
\hline $\mathrm{C}(4)-\mathrm{H}(4)$ & 0.9500 \\
\hline$C(5)-C(6)$ & $1.403(4)$ \\
\hline $\mathrm{C}(5)-\mathrm{H}(5)$ & 0.9500 \\
\hline $\mathrm{C}(6)-\mathrm{C}(7)$ & $1.488(4)$ \\
\hline $\mathrm{C}(8)-\mathrm{C}(9)$ & $1.514(4)$ \\
\hline $\mathrm{C}(8)-\mathrm{H}(8 \mathrm{~A})$ & 0.9900 \\
\hline $\mathrm{C}(8)-\mathrm{H}(8 \mathrm{~B})$ & 0.9900 \\
\hline $\mathrm{C}(9)-\mathrm{C}(10)$ & $1.509(4)$ \\
\hline$C(9)-C(11)$ & $1.531(4)$ \\
\hline$C(9)-C(12)$ & $1.533(4)$ \\
\hline $\mathrm{C}(10)-\mathrm{H}(10 \mathrm{~A})$ & 0.9900 \\
\hline $\mathrm{C}(10)-\mathrm{H}(10 \mathrm{~B})$ & 0.9900 \\
\hline $\mathrm{C}(11)-\mathrm{H}(11 \mathrm{~A})$ & 0.9800 \\
\hline $\mathrm{C}(11)-\mathrm{H}(11 \mathrm{~B})$ & 0.9800 \\
\hline $\mathrm{C}(11)-\mathrm{H}(11 \mathrm{C})$ & 0.9800 \\
\hline $\mathrm{C}(12)-\mathrm{H}(12 \mathrm{~A})$ & 0.9800 \\
\hline $\mathrm{C}(12)-\mathrm{H}(12 \mathrm{~B})$ & 0.9800 \\
\hline $\mathrm{C}(12)-\mathrm{H}(12 \mathrm{C})$ & 0.9800 \\
\hline $\mathrm{O}(2)-\mathrm{P}(1)-\mathrm{O}(4)$ & $112.45(14)$ \\
\hline $\mathrm{O}(2)-\mathrm{P}(1)-\mathrm{O}(3)$ & $113.94(15)$ \\
\hline $\mathrm{O}(4)-\mathrm{P}(1)-\mathrm{O}(3)$ & $106.75(12)$ \\
\hline
\end{tabular}




\begin{tabular}{|c|c|}
\hline $\mathrm{O}(2)-\mathrm{P}(1)-\mathrm{C}(7)$ & $112.41(14)$ \\
\hline $\mathrm{O}(4)-\mathrm{P}(1)-\mathrm{C}(7)$ & $106.49(14)$ \\
\hline $\mathrm{O}(3)-\mathrm{P}(1)-\mathrm{C}(7)$ & $104.15(13)$ \\
\hline $\mathrm{C}(8)-\mathrm{O}(3)-\mathrm{P}(1)$ & $119.55(19)$ \\
\hline $\mathrm{C}(10)-\mathrm{O}(4)-\mathrm{P}(1)$ & $122.57(19)$ \\
\hline$C(2)-C(1)-C(6)$ & $120.8(3)$ \\
\hline $\mathrm{C}(2)-\mathrm{C}(1)-\mathrm{H}(1)$ & 119.6 \\
\hline $\mathrm{C}(6)-\mathrm{C}(1)-\mathrm{H}(1)$ & 119.6 \\
\hline$C(1)-C(2)-C(3)$ & $118.8(3)$ \\
\hline $\mathrm{C}(1)-\mathrm{C}(2)-\mathrm{H}(2)$ & 120.6 \\
\hline $\mathrm{C}(3)-\mathrm{C}(2)-\mathrm{H}(2)$ & 120.6 \\
\hline$C(4)-C(3)-C(2)$ & $122.2(3)$ \\
\hline $\mathrm{C}(4)-\mathrm{C}(3)-\mathrm{Cl}(1)$ & $118.3(3)$ \\
\hline $\mathrm{C}(2)-\mathrm{C}(3)-\mathrm{Cl}(1)$ & $119.4(2)$ \\
\hline$C(3)-C(4)-C(5)$ & $118.8(3)$ \\
\hline $\mathrm{C}(3)-\mathrm{C}(4)-\mathrm{H}(4)$ & 120.6 \\
\hline $\mathrm{C}(5)-\mathrm{C}(4)-\mathrm{H}(4)$ & 120.6 \\
\hline$C(4)-C(5)-C(6)$ & $120.4(3)$ \\
\hline $\mathrm{C}(4)-\mathrm{C}(5)-\mathrm{H}(5)$ & 119.8 \\
\hline $\mathrm{C}(6)-\mathrm{C}(5)-\mathrm{H}(5)$ & 119.8 \\
\hline$C(1)-C(6)-C(5)$ & $118.9(3)$ \\
\hline$C(1)-C(6)-C(7)$ & 119.1(3) \\
\hline$C(5)-C(6)-C(7)$ & $122.0(3)$ \\
\hline $\mathrm{O}(1)-\mathrm{C}(7)-\mathrm{C}(6)$ & $122.6(3)$ \\
\hline $\mathrm{O}(1)-\mathrm{C}(7)-\mathrm{P}(1)$ & $118.1(3)$ \\
\hline $\mathrm{C}(6)-\mathrm{C}(7)-\mathrm{P}(1)$ & $119.3(2)$ \\
\hline $\mathrm{O}(3)-\mathrm{C}(8)-\mathrm{C}(9)$ & $111.3(2)$ \\
\hline $\mathrm{O}(3)-\mathrm{C}(8)-\mathrm{H}(8 \mathrm{~A})$ & 109.4 \\
\hline $\mathrm{C}(9)-\mathrm{C}(8)-\mathrm{H}(8 \mathrm{~A})$ & 109.4 \\
\hline $\mathrm{O}(3)-\mathrm{C}(8)-\mathrm{H}(8 \mathrm{~B})$ & 109.4 \\
\hline $\mathrm{C}(9)-\mathrm{C}(8)-\mathrm{H}(8 \mathrm{~B})$ & 109.4 \\
\hline $\mathrm{H}(8 \mathrm{~A})-\mathrm{C}(8)-\mathrm{H}(8 \mathrm{~B})$ & 108.0 \\
\hline$C(10)-C(9)-C(8)$ & $108.5(3)$ \\
\hline$C(10)-C(9)-C(11)$ & $107.7(3)$ \\
\hline$C(8)-C(9)-C(11)$ & $108.4(3)$ \\
\hline$C(10)-C(9)-C(12)$ & $111.9(3)$ \\
\hline$C(8)-C(9)-C(12)$ & $109.8(3)$ \\
\hline $\mathrm{C}(11)-\mathrm{C}(9)-\mathrm{C}(12)$ & $110.5(3)$ \\
\hline
\end{tabular}




$\begin{array}{ll}\mathrm{O}(4)-\mathrm{C}(10)-\mathrm{C}(9) & 111.8(2) \\ \mathrm{O}(4)-\mathrm{C}(10)-\mathrm{H}(10 \mathrm{~A}) & 109.3 \\ \mathrm{C}(9)-\mathrm{C}(10)-\mathrm{H}(10 \mathrm{~A}) & 109.3 \\ \mathrm{O}(4)-\mathrm{C}(10)-\mathrm{H}(10 \mathrm{~B}) & 109.3 \\ \mathrm{C}(9)-\mathrm{C}(10)-\mathrm{H}(10 \mathrm{~B}) & 109.3 \\ \mathrm{H}(10 \mathrm{~A})-\mathrm{C}(10)-\mathrm{H}(10 \mathrm{~B}) & 107.9 \\ \mathrm{C}(9)-\mathrm{C}(11)-\mathrm{H}(11 \mathrm{~A}) & 109.5 \\ \mathrm{C}(9)-\mathrm{C}(11)-\mathrm{H}(11 \mathrm{~B}) & 109.5 \\ \mathrm{H}(11 \mathrm{~A})-\mathrm{C}(11)-\mathrm{H}(11 \mathrm{~B}) & 109.5 \\ \mathrm{C}(9)-\mathrm{C}(11)-\mathrm{H}(11 \mathrm{C}) & 109.5 \\ \mathrm{H}(11 \mathrm{~A})-\mathrm{C}(11)-\mathrm{H}(11 \mathrm{C}) & 109.5 \\ \mathrm{H}(11 \mathrm{~B})-\mathrm{C}(11)-\mathrm{H}(11 \mathrm{C}) & 109.5 \\ \mathrm{C}(9)-\mathrm{C}(12)-\mathrm{H}(12 \mathrm{~A}) & 109.5 \\ \mathrm{C}(9)-\mathrm{C}(12)-\mathrm{H}(12 \mathrm{~B}) & 109.5 \\ \mathrm{H}(12 \mathrm{~A})-\mathrm{C}(12)-\mathrm{H}(12 \mathrm{~B}) & 109.5 \\ \mathrm{C}(9)-\mathrm{C}(12)-\mathrm{H}(12 \mathrm{C}) & 109.5 \\ \mathrm{H}(12 \mathrm{~A})-\mathrm{C}(12)-\mathrm{H}(12 \mathrm{C}) & 109.5 \\ \mathrm{H}(12 \mathrm{~B})-\mathrm{C}(12)-\mathrm{H}(12 \mathrm{C}) & 109.5 \\ \end{array}$

Symmetry transformations used to generate equivalent atoms:

Table S7. Anisotropic displacement parameters $\left(\AA^{2} \times 10^{3}\right)$ for mo_d8v21054_0m. The anisotropic displacement factor exponent takes the form: $-2 \pi^{2}\left[h^{2} a^{* 2} U^{11}+\ldots+2 h k a^{*} b^{*} U^{12}\right]$

\begin{tabular}{lllllll}
\hline & $\mathrm{U}^{11}$ & $\mathrm{U}^{22}$ & $\mathrm{U}^{33}$ & $\mathrm{U}^{23}$ & $\mathrm{U}^{13}$ & $\mathrm{U}^{12}$ \\
\hline $\mathrm{Cl}(1)$ & $54(1)$ & $38(1)$ & $43(1)$ & $-10(1)$ & $-2(1)$ & $0(1)$ \\
$\mathrm{P}(1)$ & $38(1)$ & $32(1)$ & $29(1)$ & $-3(1)$ & $1(1)$ & $2(1)$ \\
$\mathrm{O}(1)$ & $82(2)$ & $41(1)$ & $37(1)$ & $7(1)$ & $12(1)$ & $8(1)$ \\
$\mathrm{O}(2)$ & $60(2)$ & $48(1)$ & $37(1)$ & $-10(1)$ & $-11(1)$ & $16(1)$ \\
$\mathrm{O}(3)$ & $45(1)$ & $28(1)$ & $43(1)$ & $3(1)$ & $11(1)$ & $0(1)$ \\
$\mathrm{O}(4)$ & $34(1)$ & $32(1)$ & $48(1)$ & $-4(1)$ & $2(1)$ & $-3(1)$ \\
$\mathrm{C}(1)$ & $35(2)$ & $40(2)$ & $27(1)$ & $3(1)$ & $0(1)$ & $2(1)$ \\
$\mathrm{C}(2)$ & $37(2)$ & $46(2)$ & $23(1)$ & $-4(1)$ & $1(1)$ & $1(1)$ \\
$\mathrm{C}(3)$ & $30(1)$ & $37(2)$ & $34(2)$ & $-4(1)$ & $-4(1)$ & $-2(1)$ \\
$\mathrm{C}(4)$ & $43(2)$ & $37(2)$ & $26(1)$ & $1(1)$ & $-3(1)$ & $-4(1)$ \\
$\mathrm{C}(5)$ & $42(2)$ & $39(2)$ & $24(1)$ & $-1(1)$ & $-2(1)$ & $-1(1)$ \\
$\mathrm{C}(6)$ & $30(1)$ & $39(2)$ & $24(1)$ & $0(1)$ & $0(1)$ & $1(1)$
\end{tabular}




\begin{tabular}{lllllll}
$\mathrm{C}(7)$ & $35(2)$ & $40(2)$ & $26(1)$ & $2(1)$ & $2(1)$ & $2(1)$ \\
$\mathrm{C}(8)$ & $36(2)$ & $34(2)$ & $38(2)$ & $-3(1)$ & $2(1)$ & $-6(1)$ \\
$\mathrm{C}(9)$ & $37(2)$ & $30(2)$ & $28(1)$ & $0(1)$ & $0(1)$ & $-1(1)$ \\
$\mathrm{C}(10)$ & $40(2)$ & $30(2)$ & $40(2)$ & $3(1)$ & $3(1)$ & $-2(1)$ \\
$\mathrm{C}(11)$ & $42(2)$ & $49(2)$ & $45(2)$ & $6(2)$ & $-5(1)$ & $7(2)$ \\
$\mathrm{C}(12)$ & $54(2)$ & $42(2)$ & $29(2)$ & $-4(1)$ & $1(1)$ & $10(2)$ \\
\hline
\end{tabular}

Table S8. Hydrogen coordinates ( $\left.\times 10^{4}\right)$ and isotropic displacement parameters $\left(\AA^{2} \times 10^{3}\right)$ for mo_d8v21054_0m.

\begin{tabular}{lrrrl}
\hline & $\mathrm{x}$ & $\mathrm{y}$ & $\mathrm{z}$ & $\mathrm{U}(\mathrm{eq})$ \\
& & & & \\
$\mathrm{H}(1)$ & 7401 & 6865 & 4589 & 41 \\
$\mathrm{H}(2)$ & 7702 & 4722 & 4314 & 42 \\
$\mathrm{H}(4)$ & 7239 & 3853 & 6456 & 43 \\
$\mathrm{H}(5)$ & 6936 & 6000 & 6736 & 42 \\
$\mathrm{H}(8 \mathrm{~A})$ & 1592 & 8635 & 6882 & 43 \\
$\mathrm{H}(8 \mathrm{~B})$ & 2752 & 8874 & 6144 & 43 \\
$\mathrm{H}(10 \mathrm{~A})$ & 4834 & 10768 & 6017 & 44 \\
$\mathrm{H}(10 \mathrm{~B})$ & 5183 & 11686 & 6701 & 44 \\
$\mathrm{H}(11 \mathrm{~A})$ & 1618 & 11985 & 6655 & 68 \\
$\mathrm{H}(11 \mathrm{~B})$ & 188 & 10810 & 6772 & 68 \\
$\mathrm{H}(11 \mathrm{C})$ & 1359 & 10944 & 6028 & 68 \\
$\mathrm{H}(12 \mathrm{~A})$ & 4133 & 9886 & 7930 & 62 \\
$\mathrm{H}(12 \mathrm{~B})$ & 1862 & 10234 & 7913 & 62 \\
$\mathrm{H}(12 \mathrm{C})$ & 3462 & 11330 & 7854 & 62 \\
& & & & \\
\hline
\end{tabular}

Table S9. Torsion angles $\left[{ }^{\circ}\right]$ for mo_d8v21054_0m.

\begin{tabular}{lc}
\hline $\mathrm{O}(2)-\mathrm{P}(1)-\mathrm{O}(3)-\mathrm{C}(8)$ & $158.1(2)$ \\
$\mathrm{O}(4)-\mathrm{P}(1)-\mathrm{O}(3)-\mathrm{C}(8)$ & $33.4(2)$ \\
$\mathrm{C}(7)-\mathrm{P}(1)-\mathrm{O}(3)-\mathrm{C}(8)$ & $-79.0(2)$ \\
$\mathrm{O}(2)-\mathrm{P}(1)-\mathrm{O}(4)-\mathrm{C}(10)$ & $-156.0(2)$ \\
$\mathrm{O}(3)-\mathrm{P}(1)-\mathrm{O}(4)-\mathrm{C}(10)$ & $-30.4(3)$ \\
$\mathrm{C}(7)-\mathrm{P}(1)-\mathrm{O}(4)-\mathrm{C}(10)$ & $80.4(3)$
\end{tabular}




$\begin{array}{lc}\mathrm{C}(6)-\mathrm{C}(1)-\mathrm{C}(2)-\mathrm{C}(3) & -0.1(5) \\ \mathrm{C}(1)-\mathrm{C}(2)-\mathrm{C}(3)-\mathrm{C}(4) & 0.8(5) \\ \mathrm{C}(1)-\mathrm{C}(2)-\mathrm{C}(3)-\mathrm{Cl}(1) & -178.9(2) \\ \mathrm{C}(2)-\mathrm{C}(3)-\mathrm{C}(4)-\mathrm{C}(5) & -0.8(5) \\ \mathrm{Cl}(1)-\mathrm{C}(3)-\mathrm{C}(4)-\mathrm{C}(5) & 178.9(2) \\ \mathrm{C}(3)-\mathrm{C}(4)-\mathrm{C}(5)-\mathrm{C}(6) & 0.1(5) \\ \mathrm{C}(2)-\mathrm{C}(1)-\mathrm{C}(6)-\mathrm{C}(5) & -0.5(4) \\ \mathrm{C}(2)-\mathrm{C}(1)-\mathrm{C}(6)-\mathrm{C}(7) & 179.1(3) \\ \mathrm{C}(4)-\mathrm{C}(5)-\mathrm{C}(6)-\mathrm{C}(1) & 0.5(4) \\ \mathrm{C}(4)-\mathrm{C}(5)-\mathrm{C}(6)-\mathrm{C}(7) & -179.1(3) \\ \mathrm{C}(1)-\mathrm{C}(6)-\mathrm{C}(7)-\mathrm{O}(1) & -1.2(5) \\ \mathrm{C}(5)-\mathrm{C}(6)-\mathrm{C}(7)-\mathrm{O}(1) & 178.5(3) \\ \mathrm{C}(1)-\mathrm{C}(6)-\mathrm{C}(7)-\mathrm{P}(1) & 177.9(2) \\ \mathrm{C}(5)-\mathrm{C}(6)-\mathrm{C}(7)-\mathrm{P}(1) & -2.5(4) \\ \mathrm{O}(2)-\mathrm{P}(1)-\mathrm{C}(7)-\mathrm{O}(1) & -128.3(3) \\ \mathrm{O}(4)-\mathrm{P}(1)-\mathrm{C}(7)-\mathrm{O}(1) & -4.8(3) \\ \mathrm{O}(3)-\mathrm{P}(1)-\mathrm{C}(7)-\mathrm{O}(1) & 107.8(3) \\ \mathrm{O}(2)-\mathrm{P}(1)-\mathrm{C}(7)-\mathrm{C}(6) & 52.6(3) \\ \mathrm{O}(4)-\mathrm{P}(1)-\mathrm{C}(7)-\mathrm{C}(6) & 176.1(2) \\ \mathrm{O}(3)-\mathrm{P}(1)-\mathrm{C}(7)-\mathrm{C}(6) & -71.3(2) \\ \mathrm{P}(1)-\mathrm{O}(3)-\mathrm{C}(8)-\mathrm{C}(9) & -51.8(3) \\ \mathrm{O}(3)-\mathrm{C}(8)-\mathrm{C}(9)-\mathrm{C}(10) & 61.1(3) \\ \mathrm{O}(3)-\mathrm{C}(8)-\mathrm{C}(9)-\mathrm{C}(11) & 177.7(2) \\ \mathrm{O}(3)-\mathrm{C}(8)-\mathrm{C}(9)-\mathrm{C}(12) & -61.5(3) \\ \mathrm{P}(1)-\mathrm{O}(4)-\mathrm{C}(10)-\mathrm{C}(9) & 45.0(3) \\ \mathrm{C}(8)-\mathrm{C}(9)-\mathrm{C}(10)-\mathrm{O}(4) & -57.1(3) \\ \mathrm{C}(11)-\mathrm{C}(9)-\mathrm{C}(10)-\mathrm{O}(4) & -174.2(3) \\ \mathrm{C}(12)-\mathrm{C}(9)-\mathrm{C}(10)-\mathrm{O}(4) & 64.2(3) \\ & \end{array}$

Symmetry transformations used to generate equivalent atoms:

Table S10. Hydrogen bonds for mo_d8v21054_0m $\quad\left[\AA\right.$ and $\left.^{\circ}\right]$.

\begin{tabular}{lcccc}
\hline D-H...A & d(D-H) & $d(H \ldots A)$ & $d(D . . A)$ & $<($ DHA $)$ \\
\hline C(10)-H(10B)...O(2)\#1 & 0.99 & 2.59 & $3.420(4)$ & 141.1 \\
$\mathrm{C}(8)-\mathrm{H}(8 \mathrm{~B}) \ldots \mathrm{Cl}(1) \# 2$ & 0.99 & 2.84 & $3.749(3)$ & 152.8 \\
$\mathrm{C}(8)-\mathrm{H}(8 \mathrm{~A}) \ldots \mathrm{O}(2) \# 3$ & 0.99 & 2.64 & $3.625(4)$ & 172.3
\end{tabular}




\begin{tabular}{lllll}
$\mathrm{C}(4)-\mathrm{H}(4) \ldots \mathrm{O}(2) \# 4$ & 0.95 & 2.44 & $3.335(4)$ & 157.2 \\
$\mathrm{C}(10)-\mathrm{H}(10 \mathrm{~B}) \ldots \mathrm{O}(2) \# 1$ & 0.99 & 2.59 & $3.420(4)$ & 141.1 \\
$\mathrm{C}(8)-\mathrm{H}(8 \mathrm{~B}) \ldots \mathrm{Cl}(1) \# 2$ & 0.99 & 2.84 & $3.749(3)$ & 152.8 \\
$\mathrm{C}(8)-\mathrm{H}(8 \mathrm{~A}) \ldots \mathrm{O}(2) \# 3$ & 0.99 & 2.64 & $3.625(4)$ & 172.3 \\
$\mathrm{C}(4)-\mathrm{H}(4) \ldots \mathrm{O}(2) \# 4$ & 0.95 & 2.44 & $3.335(4)$ & 157.2 \\
$\mathrm{C}(4)-\mathrm{H}(4) \ldots \mathrm{O}(2) \# 4$ & 0.95 & 2.44 & $3.335(4)$ & 157.2 \\
$\mathrm{C}(8)-\mathrm{H}(8 \mathrm{~A}) \ldots \mathrm{O}(2) \# 3$ & 0.99 & 2.64 & $3.625(4)$ & 172.3 \\
$\mathrm{C}(8)-\mathrm{H}(8 \mathrm{~B}) \ldots \mathrm{Cl}(1) \# 2$ & 0.99 & 2.84 & $3.749(3)$ & 152.8 \\
$\mathrm{C}(10)-\mathrm{H}(10 \mathrm{~B}) \ldots \mathrm{O}(2) \# 1$ & 0.99 & 2.59 & $3.420(4)$ & 141.1 \\
& & & & \\
\hline
\end{tabular}

Symmetry transformations used to generate equivalent atoms:

$\# 1-\mathrm{x}+3 / 2, \mathrm{y}+1 / 2,-\mathrm{z}+3 / 2 \quad \# 2-\mathrm{x}+1,-\mathrm{y}+1,-\mathrm{z}+1 \quad \# 3 \mathrm{x}-1, \mathrm{y}, \mathrm{z}$

$\# 4-\mathrm{x}+3 / 2, \mathrm{y}-1 / 2,-\mathrm{z}+3 / 2$

\section{Crystal Structure of $\mathbf{3 b}$}

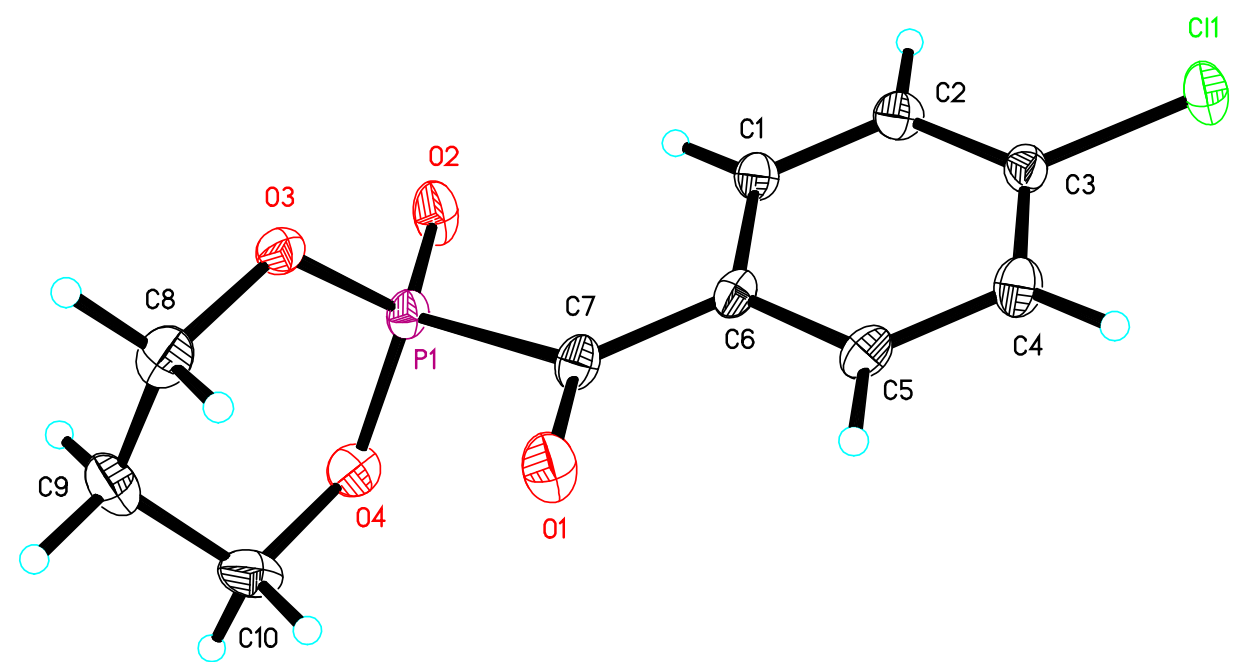

Table S11. Crystal data and structure refinement for mo_d8v21041_0m_4.

Identification code

Empirical formula

Formula weight

Temperature

Wavelength

Crystal system

Space group mo_d8v21041_0m_4

C10 H10 Cl O4 P

260.60

192(2) K

$0.71073 \AA$

Monoclinic

P 21 
Unit cell dimensions

Volume

Z

Density (calculated)

Absorption coefficient

$\mathrm{F}(000)$

Crystal size

Theta range for data collection

Index ranges

Reflections collected

Independent reflections

Completeness to theta $=25.242^{\circ}$

Absorption correction

Max. and min. transmission

Refinement method

Data / restraints / parameters

Goodness-of-fit on $\mathrm{F}^{2}$

Final $\mathrm{R}$ indices [I $>2 \operatorname{sigma}(\mathrm{I})]$

$\mathrm{R}$ indices (all data)

Absolute structure parameter

Extinction coefficient

Largest diff. peak and hole

$$
\begin{array}{ll}
\mathrm{a}=6.7410(4) \AA & \alpha=90^{\circ} . \\
\mathrm{b}=6.8006(4) \AA & \beta=92.206(2)^{\circ} . \\
\mathrm{c}=11.9412(8) \AA & \gamma=90^{\circ} .
\end{array}
$$$$
\text { 547.01(6) } \AA^{3}
$$

2

$1.582 \mathrm{Mg} / \mathrm{m}^{3}$

$0.490 \mathrm{~mm}^{-1}$

268

$0.200 \times 0.150 \times 0.110 \mathrm{~mm}^{3}$

3.024 to $25.995^{\circ}$.

$0<=\mathrm{h}<=8,-8<=\mathrm{k}<=8,-15<=\mathrm{l}<=15$

2548

$1160[\mathrm{R}(\mathrm{int})=0.0432]$

$98.4 \%$

Semi-empirical from equivalents

0.7456 and 0.5324

Full-matrix least-squares on $\mathrm{F}^{2}$

$1160 / 1 / 146$

1.199

$\mathrm{R} 1=0.0473, \mathrm{wR} 2=0.1225$

$\mathrm{R} 1=0.0576, \mathrm{wR} 2=0.1309$

$0.1(2)$

$0.05(2)$

0.358 and -0.412 e. $\AA^{-3}$

Table S12. Atomic coordinates $\left(\mathrm{x} 10^{4}\right)$ and equivalent isotropic displacement parameters $\left(\AA^{2} \mathrm{x}\right.$ $10^{3}$ ) for mo_d8v21041_0m_4. U(eq) is defined as one third of the trace of the orthogonalized $U^{\mathrm{ij}}$ tensor.

\begin{tabular}{lcccc}
\hline & $\mathrm{x}$ & $\mathrm{y}$ & $\mathrm{z}$ & $\mathrm{U}(\mathrm{eq})$ \\
\hline $\mathrm{Cl}(1)$ & $-883(2)$ & $5337(3)$ & $2763(1)$ & $44(1)$ \\
$\mathrm{P}(1)$ & $4128(2)$ & $5025(2)$ & $8225(1)$ & $27(1)$ \\
$\mathrm{O}(1)$ & $6546(5)$ & $4680(9)$ & $6601(3)$ & $45(1)$ \\
$\mathrm{O}(2)$ & $2025(5)$ & $5142(9)$ & $8463(3)$ & $43(1)$ \\
$\mathrm{O}(3)$ & $5335(6)$ & $6871(6)$ & $8645(4)$ & $30(1)$ \\
$\mathrm{O}(4)$ & $5168(7)$ & $3165(6)$ & $8750(4)$ & $37(1)$ \\
$\mathrm{C}(1)$ & $1311(7)$ & $5381(8)$ & $5900(4)$ & $28(1)$
\end{tabular}




\begin{tabular}{lrlll}
$\mathrm{C}(2)$ & $30(8)$ & $5519(8)$ & $4968(5)$ & $32(1)$ \\
$\mathrm{C}(3)$ & $758(7)$ & $5227(9)$ & $3921(4)$ & $30(1)$ \\
$\mathrm{C}(4)$ & $2747(7)$ & $4829(10)$ & $3758(4)$ & $35(1)$ \\
$\mathrm{C}(5)$ & $4016(8)$ & $4694(9)$ & $4695(4)$ & $33(1)$ \\
$\mathrm{C}(6)$ & $3322(6)$ & $4981(9)$ & $5763(4)$ & $26(1)$ \\
$\mathrm{C}(7)$ & $4763(7)$ & $4888(9)$ & $6727(4)$ & $29(1)$ \\
$\mathrm{C}(8)$ & $7429(9)$ & $6783(10)$ & $9018(6)$ & $38(2)$ \\
$\mathrm{C}(9)$ & $7817(7)$ & $4998(16)$ & $9715(4)$ & $43(1)$ \\
$\mathrm{C}(10)$ & $7272(11)$ & $3145(11)$ & $9088(6)$ & $42(2)$ \\
\hline
\end{tabular}

Table S13. Bond lengths $[\AA]$ and angles $\left[^{\circ}\right]$ for mo_d8v21041_0m_4.

\begin{tabular}{ll}
\hline $\mathrm{Cl}(1)-\mathrm{C}(3)$ & $1.739(5)$ \\
$\mathrm{P}(1)-\mathrm{O}(2)$ & $1.459(3)$ \\
$\mathrm{P}(1)-\mathrm{O}(4)$ & $1.565(5)$ \\
$\mathrm{P}(1)-\mathrm{O}(3)$ & $1.567(4)$ \\
$\mathrm{P}(1)-\mathrm{C}(7)$ & $1.858(4)$ \\
$\mathrm{O}(1)-\mathrm{C}(7)$ & $1.225(6)$ \\
$\mathrm{O}(3)-\mathrm{C}(8)$ & $1.465(8)$ \\
$\mathrm{O}(4)-\mathrm{C}(10)$ & $1.460(9)$ \\
$\mathrm{C}(1)-\mathrm{C}(2)$ & $1.385(7)$ \\
$\mathrm{C}(1)-\mathrm{C}(6)$ & $1.399(6)$ \\
$\mathrm{C}(1)-\mathrm{H}(1)$ & 0.9500 \\
$\mathrm{C}(2)-\mathrm{C}(3)$ & $1.374(7)$ \\
$\mathrm{C}(2)-\mathrm{H}(2)$ & 0.9500 \\
$\mathrm{C}(3)-\mathrm{C}(4)$ & $1.389(7)$ \\
$\mathrm{C}(4)-\mathrm{C}(5)$ & $1.385(7)$ \\
$\mathrm{C}(4)-\mathrm{H}(4)$ & 0.9500 \\
$\mathrm{C}(5)-\mathrm{C}(6)$ & $1.389(6)$ \\
$\mathrm{C}(5)-\mathrm{H}(5)$ & 0.9500 \\
$\mathrm{C}(6)-\mathrm{C}(7)$ & $1.478(6)$ \\
$\mathrm{C}(8)-\mathrm{C}(9)$ & $1.489(12)$ \\
$\mathrm{C}(8)-\mathrm{H}(8 \mathrm{~A})$ & 0.9900 \\
$\mathrm{C}(8)-\mathrm{H}(8 \mathrm{~B})$ & 0.9900 \\
$\mathrm{C}(9)-\mathrm{C}(10)$ & $1.504(12)$ \\
$\mathrm{C}(9)-\mathrm{H}(9 \mathrm{~A})$ & 0.9900 \\
$\mathrm{C}(9)-\mathrm{H}(9 \mathrm{~B})$ & 0.9900 \\
& \\
&
\end{tabular}




\begin{tabular}{|c|c|}
\hline $\mathrm{C}(10)-\mathrm{H}(10 \mathrm{~A})$ & 0.9900 \\
\hline $\mathrm{C}(10)-\mathrm{H}(10 \mathrm{~B})$ & 0.9900 \\
\hline $\mathrm{O}(2)-\mathrm{P}(1)-\mathrm{O}(4)$ & $112.9(3)$ \\
\hline $\mathrm{O}(2)-\mathrm{P}(1)-\mathrm{O}(3)$ & $113.0(3)$ \\
\hline $\mathrm{O}(4)-\mathrm{P}(1)-\mathrm{O}(3)$ & $107.46(19)$ \\
\hline $\mathrm{O}(2)-\mathrm{P}(1)-\mathrm{C}(7)$ & $116.9(2)$ \\
\hline $\mathrm{O}(4)-\mathrm{P}(1)-\mathrm{C}(7)$ & $103.2(3)$ \\
\hline $\mathrm{O}(3)-\mathrm{P}(1)-\mathrm{C}(7)$ & $102.3(3)$ \\
\hline $\mathrm{C}(8)-\mathrm{O}(3)-\mathrm{P}(1)$ & $123.1(4)$ \\
\hline $\mathrm{C}(10)-\mathrm{O}(4)-\mathrm{P}(1)$ & $122.3(4)$ \\
\hline$C(2)-C(1)-C(6)$ & $119.8(4)$ \\
\hline $\mathrm{C}(2)-\mathrm{C}(1)-\mathrm{H}(1)$ & 120.1 \\
\hline $\mathrm{C}(6)-\mathrm{C}(1)-\mathrm{H}(1)$ & 120.1 \\
\hline$C(3)-C(2)-C(1)$ & $119.2(5)$ \\
\hline $\mathrm{C}(3)-\mathrm{C}(2)-\mathrm{H}(2)$ & 120.4 \\
\hline $\mathrm{C}(1)-\mathrm{C}(2)-\mathrm{H}(2)$ & 120.4 \\
\hline$C(2)-C(3)-C(4)$ & $122.5(4)$ \\
\hline $\mathrm{C}(2)-\mathrm{C}(3)-\mathrm{Cl}(1)$ & $118.6(4)$ \\
\hline $\mathrm{C}(4)-\mathrm{C}(3)-\mathrm{Cl}(1)$ & $119.0(4)$ \\
\hline$C(5)-C(4)-C(3)$ & $117.9(4)$ \\
\hline $\mathrm{C}(5)-\mathrm{C}(4)-\mathrm{H}(4)$ & 121.0 \\
\hline $\mathrm{C}(3)-\mathrm{C}(4)-\mathrm{H}(4)$ & 121.0 \\
\hline$C(4)-C(5)-C(6)$ & $120.9(5)$ \\
\hline $\mathrm{C}(4)-\mathrm{C}(5)-\mathrm{H}(5)$ & 119.5 \\
\hline $\mathrm{C}(6)-\mathrm{C}(5)-\mathrm{H}(5)$ & 119.5 \\
\hline$C(5)-C(6)-C(1)$ & $119.8(4)$ \\
\hline$C(5)-C(6)-C(7)$ & $118.3(4)$ \\
\hline$C(1)-C(6)-C(7)$ & $121.9(4)$ \\
\hline $\mathrm{O}(1)-\mathrm{C}(7)-\mathrm{C}(6)$ & $121.9(4)$ \\
\hline $\mathrm{O}(1)-\mathrm{C}(7)-\mathrm{P}(1)$ & $112.8(3)$ \\
\hline$C(6)-C(7)-P(1)$ & $125.4(3)$ \\
\hline $\mathrm{O}(3)-\mathrm{C}(8)-\mathrm{C}(9)$ & $110.5(5)$ \\
\hline $\mathrm{O}(3)-\mathrm{C}(8)-\mathrm{H}(8 \mathrm{~A})$ & 109.6 \\
\hline $\mathrm{C}(9)-\mathrm{C}(8)-\mathrm{H}(8 \mathrm{~A})$ & 109.6 \\
\hline $\mathrm{O}(3)-\mathrm{C}(8)-\mathrm{H}(8 \mathrm{~B})$ & 109.6 \\
\hline $\mathrm{C}(9)-\mathrm{C}(8)-\mathrm{H}(8 \mathrm{~B})$ & 109.6 \\
\hline $\mathrm{H}(8 \mathrm{~A})-\mathrm{C}(8)-\mathrm{H}(8 \mathrm{~B})$ & 108.1 \\
\hline$C(8)-C(9)-C(10)$ & $111.8(4)$ \\
\hline
\end{tabular}




$\begin{array}{ll}\mathrm{C}(8)-\mathrm{C}(9)-\mathrm{H}(9 \mathrm{~A}) & 109.3 \\ \mathrm{C}(10)-\mathrm{C}(9)-\mathrm{H}(9 \mathrm{~A}) & 109.3 \\ \mathrm{C}(8)-\mathrm{C}(9)-\mathrm{H}(9 \mathrm{~B}) & 109.3 \\ \mathrm{C}(10)-\mathrm{C}(9)-\mathrm{H}(9 \mathrm{~B}) & 109.3 \\ \mathrm{H}(9 \mathrm{~A})-\mathrm{C}(9)-\mathrm{H}(9 \mathrm{~B}) & 107.9 \\ \mathrm{O}(4)-\mathrm{C}(10)-\mathrm{C}(9) & 110.2(5) \\ \mathrm{O}(4)-\mathrm{C}(10)-\mathrm{H}(10 \mathrm{~A}) & 109.6 \\ \mathrm{C}(9)-\mathrm{C}(10)-\mathrm{H}(10 \mathrm{~A}) & 109.6 \\ \mathrm{O}(4)-\mathrm{C}(10)-\mathrm{H}(10 \mathrm{~B}) & 109.6 \\ \mathrm{C}(9)-\mathrm{C}(10)-\mathrm{H}(10 \mathrm{~B}) & 109.6 \\ \mathrm{H}(10 \mathrm{~A})-\mathrm{C}(10)-\mathrm{H}(10 \mathrm{~B}) & 108.1\end{array}$

Symmetry transformations used to generate equivalent atoms:

Table S14. Anisotropic displacement parameters $\left(\AA^{2} \times 10^{3}\right)$ for mo_d8v21041_0m_4. The anisotropic displacement factor exponent takes the form: $\quad-2 \pi^{2}\left[h^{2} a^{* 2} U^{11}+\ldots+2 h k a^{*} b^{*} U^{12}\right]$

\begin{tabular}{|c|c|c|c|c|c|c|}
\hline & $\mathrm{U}^{11}$ & $\mathrm{U}^{22}$ & $\mathrm{U}^{33}$ & $\mathrm{U}^{23}$ & $\mathrm{U}^{13}$ & $\mathrm{U}^{12}$ \\
\hline $\mathrm{Cl}(1)$ & $50(1)$ & $55(1)$ & $27(1)$ & $3(1)$ & $-6(1)$ & $1(1)$ \\
\hline $\mathrm{P}(1)$ & $26(1)$ & $37(1)$ & $19(1)$ & $-2(1)$ & $7(1)$ & $-2(1)$ \\
\hline $\mathrm{O}(1)$ & $27(2)$ & $81(4)$ & $29(2)$ & $-3(2)$ & $8(1)$ & $0(2)$ \\
\hline $\mathrm{O}(2)$ & $28(2)$ & $75(3)$ & $27(2)$ & $-4(3)$ & $4(1)$ & $-3(3)$ \\
\hline $\mathrm{O}(3)$ & $29(2)$ & $31(2)$ & $31(2)$ & $-5(2)$ & $2(2)$ & $3(2)$ \\
\hline $\mathrm{O}(4)$ & $44(3)$ & $33(2)$ & $33(3)$ & $5(2)$ & $6(2)$ & $-1(2)$ \\
\hline$C(1)$ & $32(2)$ & $33(3)$ & $21(2)$ & $4(2)$ & $7(2)$ & $1(2)$ \\
\hline$C(2)$ & $31(2)$ & $33(3)$ & $31(2)$ & $3(2)$ & $7(2)$ & $0(2)$ \\
\hline$C(3)$ & $41(3)$ & $24(3)$ & $25(2)$ & $1(3)$ & $-2(2)$ & $-1(3)$ \\
\hline$C(4)$ & $39(3)$ & $41(3)$ & $24(2)$ & $2(3)$ & $8(2)$ & $-4(3)$ \\
\hline$C(5)$ & $34(2)$ & $35(3)$ & $29(2)$ & $-5(2)$ & $11(2)$ & $-1(2)$ \\
\hline$C(6)$ & $34(2)$ & $24(2)$ & $22(2)$ & $-3(2)$ & $5(2)$ & $-4(2)$ \\
\hline$C(7)$ & $32(2)$ & $35(3)$ & $22(2)$ & $-2(3)$ & $7(2)$ & $-5(3)$ \\
\hline$C(8)$ & $25(3)$ & $45(4)$ & $45(4)$ & $-10(3)$ & $8(3)$ & $-8(3)$ \\
\hline$C(9)$ & $25(2)$ & $78(4)$ & $26(2)$ & $5(4)$ & $5(2)$ & $8(4)$ \\
\hline$C(10)$ & $43(4)$ & $48(4)$ & $35(4)$ & $14(3)$ & $13(3)$ & $17(3)$ \\
\hline
\end{tabular}


Table S15. Hydrogen coordinates ( x 104) and isotropic displacement parameters $\left(\AA^{2} \times 10^{3}\right)$ for mo_d8v21041_0m_4.

\begin{tabular}{lrrrr}
\hline & $\mathrm{x}$ & $\mathrm{y}$ & $\mathrm{z}$ & $\mathrm{U}(\mathrm{eq})$ \\
\hline $\mathrm{H}(1)$ & & & & \\
$\mathrm{H}(2)$ & 825 & 5558 & 6629 & 34 \\
$\mathrm{H}(4)$ & -1334 & 5812 & 5051 & 38 \\
$\mathrm{H}(5)$ & 3223 & 4654 & 3026 & 42 \\
$\mathrm{H}(8 \mathrm{~A})$ & 5379 & 4402 & 4606 & 39 \\
$\mathrm{H}(8 \mathrm{~B})$ & 7777 & 7972 & 9461 & 46 \\
$\mathrm{H}(9 \mathrm{~A})$ & 8273 & 6753 & 8357 & 46 \\
$\mathrm{H}(9 \mathrm{~B})$ & 7039 & 5078 & 10400 & 51 \\
$\mathrm{H}(10 \mathrm{~A})$ & 9241 & 4953 & 9948 & 51 \\
$\mathrm{H}(10 \mathrm{~B})$ & 8081 & 3035 & 8416 & 50 \\
& 7560 & 1991 & 9572 & 50 \\
\hline
\end{tabular}

Table S16. Torsion angles $\left[^{\circ}\right]$ for mo_d8v21041_0m_4.

\begin{tabular}{lc}
\hline $\mathrm{O}(2)-\mathrm{P}(1)-\mathrm{O}(3)-\mathrm{C}(8)$ & $149.1(5)$ \\
$\mathrm{O}(4)-\mathrm{P}(1)-\mathrm{O}(3)-\mathrm{C}(8)$ & $23.9(5)$ \\
$\mathrm{C}(7)-\mathrm{P}(1)-\mathrm{O}(3)-\mathrm{C}(8)$ & $-84.4(5)$ \\
$\mathrm{O}(2)-\mathrm{P}(1)-\mathrm{O}(4)-\mathrm{C}(10)$ & $-150.8(5)$ \\
$\mathrm{O}(3)-\mathrm{P}(1)-\mathrm{O}(4)-\mathrm{C}(10)$ & $-25.5(5)$ \\
$\mathrm{C}(7)-\mathrm{P}(1)-\mathrm{O}(4)-\mathrm{C}(10)$ & $82.1(5)$ \\
$\mathrm{C}(6)-\mathrm{C}(1)-\mathrm{C}(2)-\mathrm{C}(3)$ & $1.0(9)$ \\
$\mathrm{C}(1)-\mathrm{C}(2)-\mathrm{C}(3)-\mathrm{C}(4)$ & $-1.1(9)$ \\
$\mathrm{C}(1)-\mathrm{C}(2)-\mathrm{C}(3)-\mathrm{Cl}(1)$ & $178.2(4)$ \\
$\mathrm{C}(2)-\mathrm{C}(3)-\mathrm{C}(4)-\mathrm{C}(5)$ & $1.1(9)$ \\
$\mathrm{Cl}(1)-\mathrm{C}(3)-\mathrm{C}(4)-\mathrm{C}(5)$ & $-178.3(5)$ \\
$\mathrm{C}(3)-\mathrm{C}(4)-\mathrm{C}(5)-\mathrm{C}(6)$ & $-1.0(9)$ \\
$\mathrm{C}(4)-\mathrm{C}(5)-\mathrm{C}(6)-\mathrm{C}(1)$ & $1.0(9)$ \\
$\mathrm{C}(4)-\mathrm{C}(5)-\mathrm{C}(6)-\mathrm{C}(7)$ & $-177.9(6)$ \\
$\mathrm{C}(2)-\mathrm{C}(1)-\mathrm{C}(6)-\mathrm{C}(5)$ & $-1.0(9)$ \\
$\mathrm{C}(2)-\mathrm{C}(1)-\mathrm{C}(6)-\mathrm{C}(7)$ & $177.8(6)$ \\
$\mathrm{C}(5)-\mathrm{C}(6)-\mathrm{C}(7)-\mathrm{O}(1)$ & $4.2(9)$ \\
$\mathrm{C}(1)-\mathrm{C}(6)-\mathrm{C}(7)-\mathrm{O}(1)$ & $-174.6(6)$
\end{tabular}


$\mathrm{O}(3)-\mathrm{C}(8)-\mathrm{C}(9)-\mathrm{C}(10)$

Symmetry transformations used to generate equivalent atoms:

Table S17. Hydrogen bonds for mo_d8v21041_0m_4 [A and $\left.{ }^{\circ}\right]$.

\begin{tabular}{lcccc}
\hline D-H...A & $d(\mathrm{D}-\mathrm{H})$ & $\mathrm{d}(\mathrm{H} \ldots \mathrm{A})$ & $\mathrm{d}(\mathrm{D} \ldots \mathrm{A})$ & $<(\mathrm{DHA})$ \\
\hline $\mathrm{C}(10)-\mathrm{H}(10 \mathrm{~A}) \ldots \mathrm{O}(1)$ & 0.99 & 2.62 & $3.168(8)$ & 115.2 \\
$\mathrm{C}(9)-\mathrm{H}(9 \mathrm{~B}) \ldots \mathrm{O}(2) \# 1$ & 0.99 & 2.63 & $3.259(6)$ & 121.1 \\
$\mathrm{C}(2)-\mathrm{H}(2) \ldots \mathrm{O}(1) \# 2$ & 0.95 & 2.50 & $3.162(7)$ & 126.5 \\
\hline
\end{tabular}

Symmetry transformations used to generate equivalent atoms:

$\# 1 \mathrm{x}+1, \mathrm{y}, \mathrm{z} \quad \# 2 \mathrm{x}-1, \mathrm{y}, \mathrm{z}$ 


\section{References}

[1] Armarego, W. L. F.; Perrin, D. D. Purification of Laboratory Chemicals. 8th Edition, Butterworth-Heinemann, Oxford, 2017.

[2] Chen, C.; Luo, Y.; Fu, L.; Chen, P.; Lan, Y.; Liu, G. J. Am. Chem. Soc. 2018, 140, $1207-1210$.

[3] Durel, V.; Lalli, C.; Roisnel, T.; van de Weghe, P. J. Org. Chem. 2016, 81, 849-859

[4] Boeckman, R. K. Jr; Charette, A. B.; Asberom, T.; Johnston, B. H. J. Am. Chem. Soc. 1991, 113, 5337-5353

[5] Zheng, D.; Studer, A. Org. Lett. 2019, 21, 325-329.

[6] Wang, X.; Ye, Y.; Zhang, S.; Feng, J.; Xu, Y.; Zhang, Y.; Wang, J. J. Am. Chem. Soc. 2011, 133, 16410-16413.

[7] Beauchamp, L. M.; Tuttle, J. V.; Rodriguez, M. E.; Sznaidman, M. L. J. Med. Chem. 1996, 39, 949-956.

[8] Chen, X.; Ning, X.; Kang, Y. Org. Lett. 2016, 18, 5368-5371.

[9] Xu, G.-Z; Micklatcher, M.; Silvestri, M. A.; Hartman, T. L.; Burrier, J.; Osterling, M. C.; Wargo, H.; Turpin, J. A.; Buckheit, Jr., R. W.; Cushman, M. J. Med. Chem. 2001, 44, 4092-4113.

[10] Li, Z.; Zhang, C.; Zhu, L.; Liu, C.; Li C. Org. Chem. Front. 2014, 1, 100-104.

[11] Zhang, Z.; Zhu, L.; Li, C. Chin. J. Chem. 2019, 37, 452-456.

[12] Lerch, M. M.; Morandi, B.; Wickens, Z. K.; Grubbs, R. H. Angew. Chem., Int. Ed. 2014, 53, 8654-8658.

[13] Wang, Q. Q.; Luo, N.; Wang, X. D.; Ao, Y. F.; Chen, Y. F.; Liu, J. M.,; Su, C. Y.; Wang, D. X.; Wang, M. X. J. Am. Chem. Soc. 2017, 139, 635-638.

[14] Qi, X.; Yu, F.; Chen, P.; Liu, G. Angew. Chem. Int. Ed. 2017, 56, 12692-12696.

[15] Fadel, A.; Salauen, J.; Conia, J. M. Tetrahedron 1983, 39, 1567-1573.

[16] Xiao, H.; Shen, H.; Zhu, L.; Li, C. J. Am. Chem. Soc. 2019, 141, 11440-11445.

[17] Liu, Y.; Wu, H.; Guo, Y.; Xiao, J. C.; Chen, Q. Y.; Liu, C. Angew. Chem., Int. Ed. 2017, 56, 15432-15435.

[18] Huang, Y.; Berthiol, F.; Stegink, B.; Pollard, M. M.; Minnaarda, A. J. Adv. Synth. 
Catal. 2009, 351, 1423-1430.

[19] Sprecher, M.; Kost, D. J. Am. Chem. Soc. 1994, 116, 1016-1026.

[20] Fischetti, W.; Heck, R. F. J. Organomet. Chem. 1985, 293, 391-405

[21] Oliveira, C. C.; dos Santos, E. A. F.; Nunes, J. H. B.; Correia, C. R. D. J. Org. Chem. 2012, 77, 8182-8190.

[22] Ma, G.; Wan, W.; Li, J.; Hu, Q.; Jiang, H.; Zhu, S.; Wang, J.; Hao, J. Chem. Commun. 2014, 50, 9749-9752.

[23] Muthiah, C.; Kumar, K. P.; Mani, C. A.; Swamy, K. C. K. J. Org. Chem. 2000, 65, $3733-3737$.

[24] Munoz, A.; Hubert, C.; Luche, J.-L. J. Org. Chem. 1996, 61, 6015-6017.

[25] Kehler, J.; Ebert, B.; Dahl, O.; Larsen-K. P. Tetrahedron 1999, 55, 771-780.

[26] Bourumeau, K.; Gaumont, A.-C.; Denis, J.-M. J. Organomet. Chem. 1997, 529, 205-213 